\title{
ADVANCED MEASUREMENTS AND ANALYSES OF FLOW PAST THREE-CYLINDER ROTATING SYSTEM
}

\author{
A Thesis \\ Submitted to the Graduate Faculty \\ of the \\ North Dakota State University \\ of Agriculture and Applied Science
}

By

Al Habib Ullah

In Partial Fulfillment of the Requirements

For the Degree of

MASTER OF SCIENCE

Major Department:

Mechanical Engineering

April 2020

Fargo, North Dakota 


\section{North Dakota State University \\ Graduate School}

Title

ADVANCED MEASUREMENTS AND ANALYSES OF FLOW PAST

THREE-CYLINDER ROTATING SYSTEM

By

Al Habib Ullah

The Supervisory Committee certifies that this disquisition complies with North Dakota State

University's regulations and meets the accepted standards for the degree of

\section{MASTER OF SCIENCE}

SUPERVISORY COMMITTEE:

Dr. Jordi Estevadeordal

Chair

Dr. Bora Suzen

Dr. Yan Zhang

Dr. Friedrich Littmann

Approved:

$4 / 15 / 20$

Date
Dr. Alan Kallmeyer

Department Chair 


\begin{abstract}
Interaction of flow structures from a three-cylinder system is complex and important for fundamental and engineering applications. In this study, experiments using hotwire, 2D PIV, and Tomography are to be conducted to characterize the fluid flow at various Re number and rotation speeds. The Reynolds number considered based on the diameter of the single-cylinder ranges from 37 to 1700 . The peaks in the frequency spectrum obtained from the hotwire study show a unique relation of Strouhal number as a function of static incident angle, RPM, and Reynolds number. From the 2D PIV and 3D tomography experiment, vorticity and velocity results characterize the interaction of wake flow from individual cylinders and as a function of the rotational speeds. Besides, the Standard deviation map shows the turbulence intensity variation at the various static and rotating conditions. The obtained results at static conditions are found to be consistent with the previous computational study.
\end{abstract}




\section{ACKNOWLEDGEMENTS}

I would like to thank my committee members for their time and effort. Special thanks to my advisor Dr. Jordi Estevadeordal for his valuable suggestion and all the support. I would also like to thank Dr. Bora Suzen, Dr. Yan Zhang and Dr. Friedrich Littmann for their valueable suggestions.

Next, I would like to thank my colleague Charles Fabijanic, Nick Thomas, Jiaming Zhao and Bailey Carson for their help. 


\section{DEDICATION}

Dedicated to my Parents for all their love and encouragement throughout my education. 


\section{TABLE OF CONTENTS}

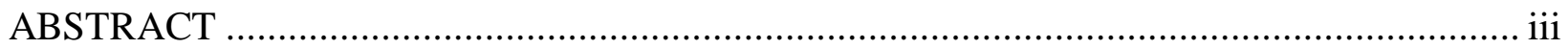

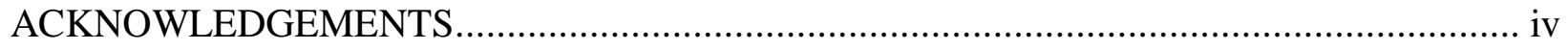

DEDICATION

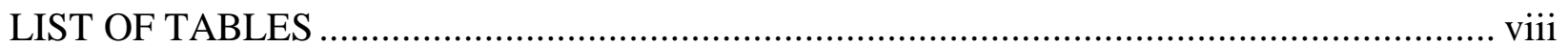

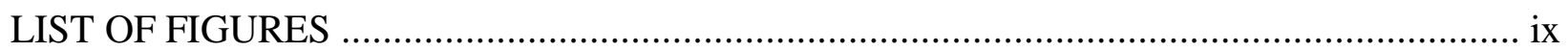

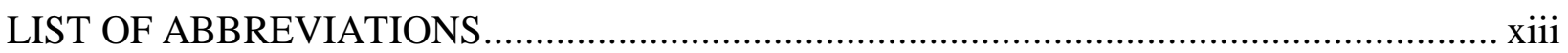

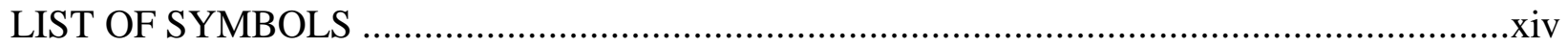

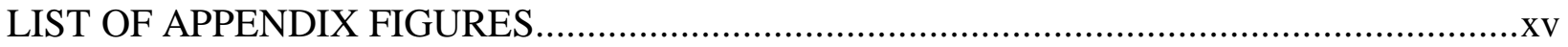

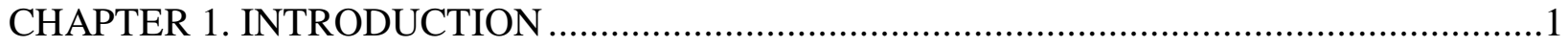

Purpose

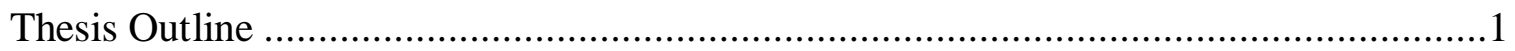

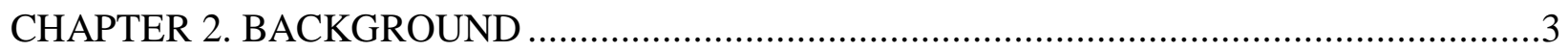

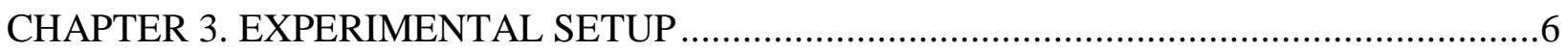

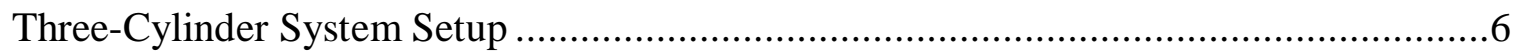

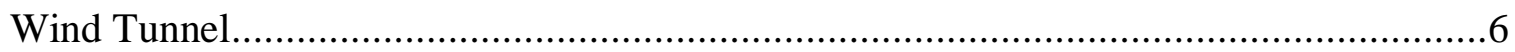

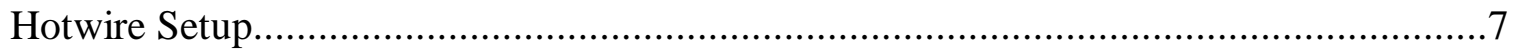

Particle Image Velocimetry (PIV) Setup ..................................................................

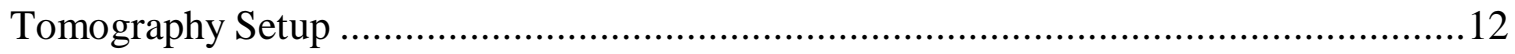

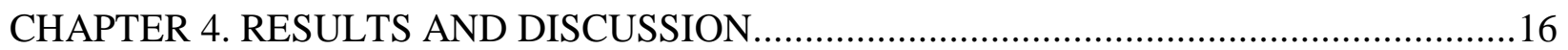

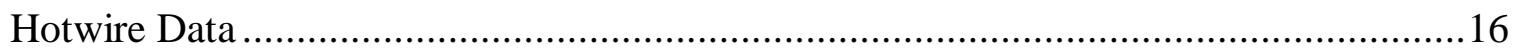

Non-Rotating Cases ................................................................................. 17

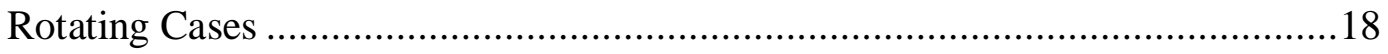

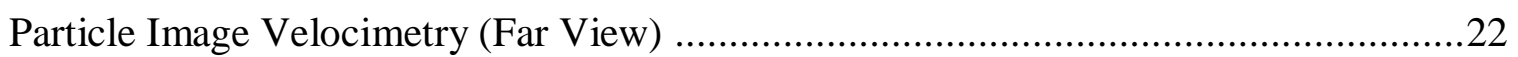

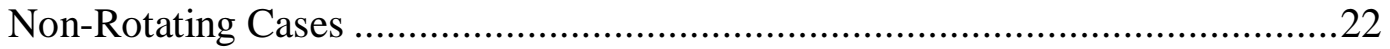


Rotating Cases: Velocity Contour and Wake Velocity Profile …….......................36

Rotating Cases: Vorticity Contour and Standard Deviation ................................44

Particle Image Velocimetry (Close View) ……….......................................................52

Non-Rotating Cases: Flow Visualization ....................................................52

Non-Rotating Cases: Instantaneous and Average Velocity Contour.....................54

Rotating Cases: Insantaneous Velocity and Vorticty Contour ..............................61

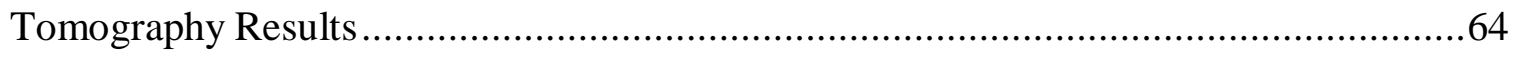

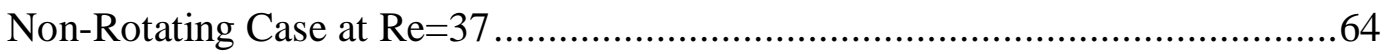

Uncertainty of Experiment .....................................................................................

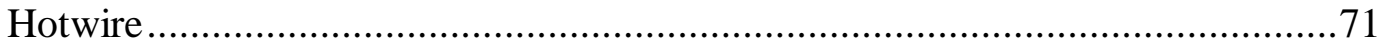

2D Particle Image Velocimetry ......................................................................

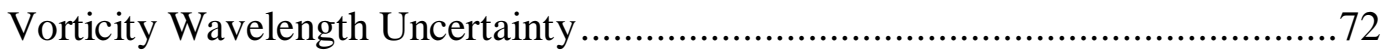

CHAPTER 5. PROPER ORTHOGONAL DECOMPOSITION (POD) ANALYSIS ..................73

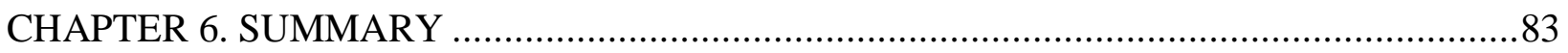

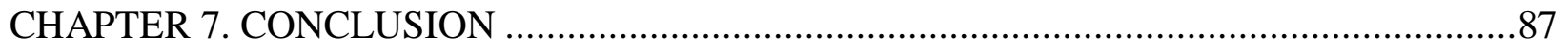

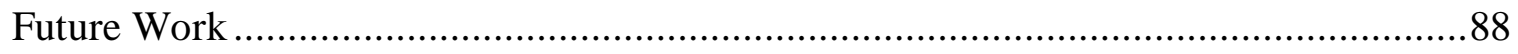

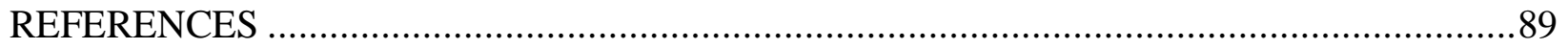

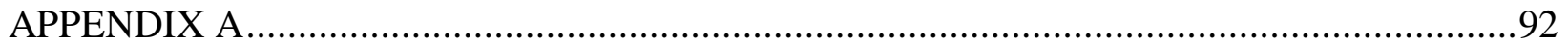

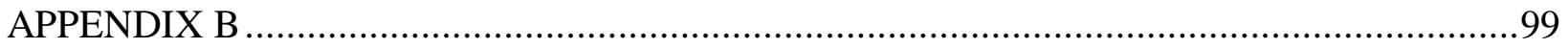

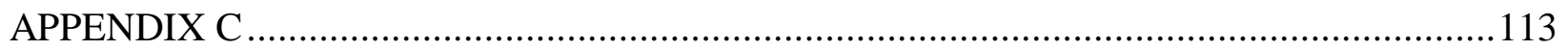

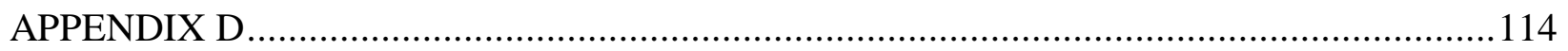

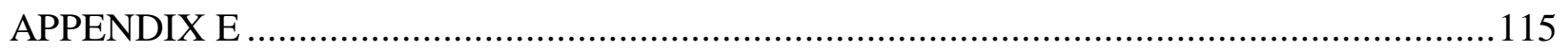

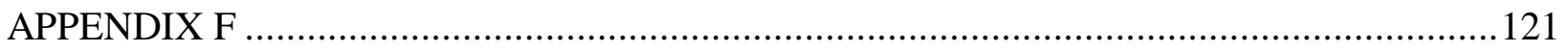

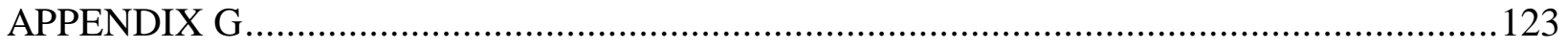

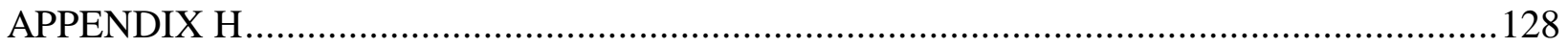




\section{LIST OF TABLES}

$\underline{\text { Table }}$

$\underline{\text { Page }}$

1. Summary of Stouhal number for different orientation of cylinder system at Position (a) and (b) 18 


\section{LIST OF FIGURES}

$\underline{\text { Figure }}$

$\underline{\text { Page }}$

1. Three-cylinder Setup (a) installed in wind tunnel (b) Motor drive to rotate the three-cylinder setup 6

2. NDSU's FloTek 1440 wind tunnel and workstation .7

3. Hotwire calibration setup using Pitot tube (a) Calibration between voltage and velocity (b). 8

4. Hotwire positioned very close to the three cylinder system (a) far from the cylinder system (b)

5. Schematic of Standard PIV system 9

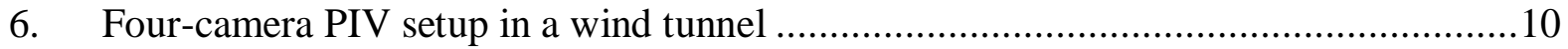

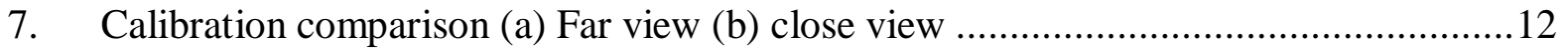

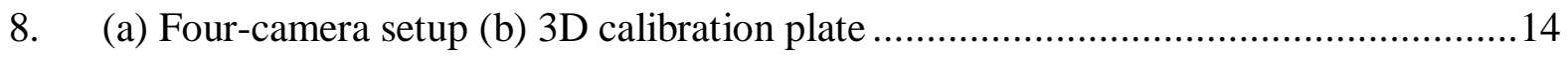

9. LASER illumination during Tomography Testing ................................................. 15

10. Schematic positions of hotwires to obtain the time resolved data ............................... 16

11. Strouhal Number plot with varying Reynolds number at Incident Angle $180^{\circ}$ and $45^{\circ}$ when the hotwire was placed at $\mathrm{x} / \mathrm{d}=1.61, \mathrm{y} / \mathrm{d}=2.21$, and $\mathrm{x} / \mathrm{d}=1.61, \mathrm{y} / \mathrm{d}=0$ respectively.

12. Strouhal Number plot as function of rotation for (a) $R e=57$ for three different location of hotwire probe, (b) different Reynolds number when the hotwire probe was located at position-a, (c) when the hotwire was located at position $-\mathrm{b}$, (c) when the hotwire was located at position-c

13. Instantaneous Vorticity contour at $\mathrm{Re}=57$ and Incident Angle (a) $0^{\circ}$, (b) $45^{0}$ and (c) $180^{\circ}$

14. Instantaneous Vorticity contour at $\mathrm{Re}=500$ and Incident Angle (a) $0^{\circ}$, (b) $45^{\circ}$, and (c) $180^{\circ}$

15. Instantaneous Vorticity contour at $\mathrm{Re}=858$ and Incident Angle (a) $0^{\circ}$, (b) $45^{\circ}$, and (c) $180^{\circ}$

16. Instantaneous Vorticity contour at $\mathrm{Re}=1250$ and Incident Angle (a) $0^{\circ}$, (b) $45^{\circ}$, and (c) $180^{\circ}$

17. Variation of vorticity diameter with respect to Re number .28 
18. Standard Deviation contour at $\mathrm{Re}=57$ and Incident Angle (a) $0^{\circ}$, (b) $45^{\circ}$, and (c) $180^{\circ}$

19. Standard Deviation at $\mathrm{Re}=500$ and Incident Angle (a) $0^{0}$, (b) $45^{\circ}$, and (c) $180^{\circ} \ldots \ldots \ldots .29$

20. Standard Deviation at $\mathrm{Re}=858$ and Incident Angle (a) $0^{\circ}$, (b) $45^{\circ}$, and (c) $180^{\circ} \ldots \ldots \ldots .29$

21. Standard Deviation at $\mathrm{Re}=1250$ and Incident Angle (a) $0^{\circ}$, (b) $45^{\circ}$, and (c) $180^{\circ} \ldots \ldots . .30$

22. Standard Deviation at $\mathrm{Re}=1700$ and Incident Angle (a) $0^{\circ}$, (b) $45^{\circ}$, and (c) $180^{\circ} \ldots \ldots . .30$

23. Average Streamwise velocity profile for $\mathrm{Re}=57$ at position (a) $\mathrm{x} / \mathrm{d}=0$,

(b) $\mathrm{x} / \mathrm{d}=12.9$, (c) $\mathrm{x} / \mathrm{d}=25.8$, and (d) 38.7 31

24. Average Streamwise velocity profile for $\mathrm{Re}=500$ at position (a) $\mathrm{x} / \mathrm{d}=0$,

(b) $\mathrm{x} / \mathrm{d}=12.9$, (c) $\mathrm{x} / \mathrm{d}=25.8$, and (d) 38.7

25. Average Streamwise velocity profile for $\mathrm{Re}=858$ at position (a) $\mathrm{x} / \mathrm{d}=0$,

(b) $\mathrm{x} / \mathrm{d}=12.9$, (c) $\mathrm{x} / \mathrm{d}=25.8$, and (d) 38.7

26. Average Streamwise velocity profile for $\mathrm{Re}=1250$ at position (a) $\mathrm{x} / \mathrm{d}=0$,

(b) $\mathrm{x} / \mathrm{d}=12.9$, (c) $\mathrm{x} / \mathrm{d}=25.8$, and (d) 38.7

27. Average Streamwise velocity profile for $\mathrm{Re}=1700$ at position (a) $\mathrm{x} / \mathrm{d}=0$,

(b) $\mathrm{x} / \mathrm{d}=12.9$, (c) $\mathrm{x} / \mathrm{d}=25.8$, and (d) 38.7

28. Instantaneous Velocity Contour at $\mathrm{Re}=57$ and Rotation Speed is (a) 120

(b) 360 (c) 960 (d) 1500 (e) 1800 (f) 2400 (g) 3000 RPM .38

29. Velocity profile for $\mathrm{Re}=57$ at location (a) $\mathrm{x} / \mathrm{D}=0$ (b) $\mathrm{x} / \mathrm{D}=4.13$ (c) $\mathrm{x} / \mathrm{D}=8.26$

(d) $\mathrm{x} / \mathrm{D}=12.4$

30. Velocity profile for $\mathrm{Re}=500$ at location (a) $\mathrm{x} / \mathrm{D}=0$ (b) $\mathrm{x} / \mathrm{D}=4.13$ (c) $\mathrm{x} / \mathrm{D}=8.26$

(d) $\mathrm{x} / \mathrm{D}=12.4$

31. Velocity profile for $\mathrm{Re}=858$ at location (a) $\mathrm{x} / \mathrm{D}=0$ (b) $\mathrm{x} / \mathrm{D}=4.13$ (c) $\mathrm{x} / \mathrm{D}=8.26$

(d) $\mathrm{x} / \mathrm{D}=12.4$

32. Velocity profile for $\mathrm{Re}=1258$ at location (a) $\mathrm{x} / \mathrm{D}=0$ (b) $\mathrm{x} / \mathrm{D}=4.13$ (c) $\mathrm{x} / \mathrm{D}=8.26$

(d) $\mathrm{x} / \mathrm{D}=12.4$

33. Velocity profile for $\mathrm{Re}=1700$ at location (a) $\mathrm{x} / \mathrm{D}=0$ (b) $\mathrm{x} / \mathrm{D}=4.13$ (c) $\mathrm{x} / \mathrm{D}=8.26$

(d) $\mathrm{x} / \mathrm{D}=12.4$

34. Instantaneous Vorticity Contour at $\mathrm{Re}=57$ and Rotation Speed is (a) 120

(b) 360 (c) 960 (d) 1500 (e) 1800 (f) 2400 (g) 3000 RPM

35. Instantaneous Vorticity Contour at $\mathrm{Re}=858$ and Rotation Speed is (a) 120

(b) 360 (c) 960 (d) 1500 (e) 1800 (f) 2400 (g) 3000 RPM 
36. Standard Deviation at $\mathrm{Re}=57$ and Rotation Speed is (a) 120 (b) 360

(c) 960 (d) 1500 (e) 1800 (f) 2400 (g) 3000 RPM

37. Standard deviation at $\mathrm{Re}=1700$ and Rotation Speed is (a) 120 (b) 360

(c) 960 (d) 1500 (e) 1800 (f) 2400 (g) 3000 RPM

38. Instantaneous vorticity plot when the cylinder orientation was (a) $0^{0}$

(b) $180^{\circ}$ at $\mathrm{Re}=57$ rotation $\mathrm{RPM}=120 \mathrm{~Hz}$ .50

39. Instantaneous vorticity plot when the cylinder orientation was (a) $0^{0}$

(b) $180^{\circ}$ at $\mathrm{Re}=57$ rotation $\mathrm{RPM}=360 \mathrm{~Hz}$.

40. Flow visualization at $\mathrm{Re}=37$ and Static Angle $0^{0}$ .53

41. Flow visualization at $\mathrm{Re}=37$ and Static Angle $180^{\circ}$

42. Instantaneous velocity contour at $\mathrm{Re}=37$ and incident angle

(a) $0^{\circ}$ (b) $45^{\circ}$ and (c) $90^{\circ}$

43. Instantaneous vorticity contour at $\mathrm{Re}=37$ and incident angle

(a) $0^{\circ}$ (b) $45^{\circ}$ and (c) $90^{\circ}$

44. Instantaneous velocity contour at $\mathrm{Re}=57$ and incident angle

(a) $0^{\circ}$ (b) $45^{\circ}$ and (c) $90^{\circ}$

45. Instantaneous velocity contour at $\mathrm{Re}=500$ and incident angle

(a) $0^{0}$ (b) $45^{\circ}$ and (c) $90^{\circ}$

46. Instantaneous velocity contour at $\mathrm{Re}=858$ and incident angle

(a) $0^{\circ}$ (b) $45^{\circ}$ and (c) $90^{\circ}$

47. Instantaneous velocity contour at $\mathrm{Re}=1250$ and incident angle

(a) $0^{\circ}$ (b) $45^{\circ}$ and (c) $90^{\circ}$

48. Instantaneous velocity contour at $\mathrm{Re}=1700$ and incident angle

(a) $0^{\circ}$ (b) $45^{\circ}$ and (c) $90^{\circ}$

49. Average velocity contour at $\mathrm{Re}=57$ and incident angle

(a) $0^{\circ}$ (b) $45^{\circ}$ and (c) $90^{\circ}$ .58

50. Average velocity contour at $\mathrm{Re}=500$ and incident angle

(a) $0^{\circ}$ (b) $45^{\circ}$ and (c) $90^{\circ}$

51. Average velocity contour at $\mathrm{Re}=858$ and incident angle (a) $0^{0}$ (b) $45^{\circ}$ and (c) $90^{\circ} \ldots \ldots .59$

52. Average velocity contour at $\operatorname{Re}=1250$ and incident angle (a) $0^{\circ}$ (b) $45^{\circ}$ and (c) $90^{\circ} \ldots .60$

53. Average velocity contour at $\operatorname{Re}=1700$ and incident angle (a) $0^{\circ}$ (b) $45^{\circ}$ and (c) $90^{\circ} \ldots .60$

54. Instantaneous Velocity Contour at $\mathrm{Re}=57$ and Rotation Speed is (a) 120

(b) 360 (c) 960 (d) 1500 (e) 1800 (f) 2400 (g) 3000 RPM 
55. Instantaneous Vorticity Contour at $\mathrm{Re}=57$ and Rotation Speed is (a) 120

(b) 360 (c) 960 (d) 1500 (e) 1800 (f) 2400 (g) 3000 RPM

56. Vorticity contour at $\mathrm{Z}=-2.5,0$, and $2.5 \mathrm{~mm}$ for $\mathrm{Re}=37$ at incident angle $0^{0}$. .65

57. Vorticity contour at $\mathrm{Z}=-2.5,0$, and $2.5 \mathrm{~mm}$ for $\mathrm{Re}=37$ at incident angle $45^{\circ} \ldots \ldots \ldots \ldots . .66$

58. Vorticity contour at $\mathrm{Z}=-2.5,0$, and $2.5 \mathrm{~mm}$ for $\mathrm{Re}=37$ at incident angle $180^{\circ} \ldots \ldots \ldots \ldots 6$

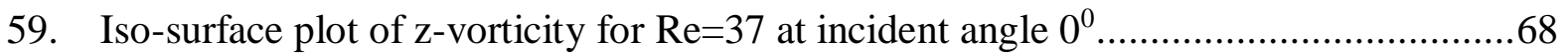

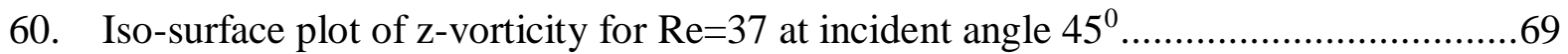

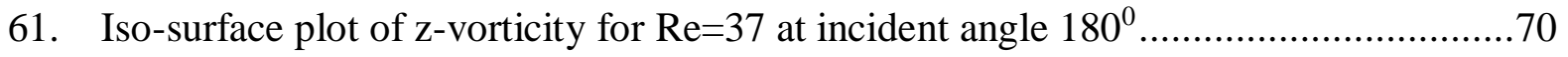

62. Relative Energy for 100 POD modes (a) Static at $0^{0}$ incident angle

(b) Static at $180^{\circ}$ incident angle

63. POD modes 1-10,80, 90 (from left to right) for Static $0^{0}$ Incident angle at $\mathrm{Re}=57$.

64. POD modes 1-10,80, 90 (from left to right) for Static $180^{\circ}$ Incident angle at $\mathrm{Re}=57$

65. First six (left to right) reconstructed velocity contour based on 10 POD modes for Static $0^{0}$ incident Angle at $\mathrm{Re}=57$.

66. First six (left to right) reconstructed velocity contour based on 10 POD modes for Static $180^{\circ}$ incident Angle at $\mathrm{Re}=57$.

67. Comparison of first three PIV instantaneous velcoity contour (a) with Reconstructed velocity contour based on POD analysis for $0^{0}$ incident angle at $\mathrm{Re}=57$

68. Comparison of first three PIV instantaneous velcoity contour (a) with Reconstructed velocity contour based on POD analysis for $180^{\circ}$ incident angle at $\mathrm{Re}=57$..............82

69. Schematics of flow structrue for static cases (a) $0^{\circ}$, (b) $45^{\circ}$, and (c) $180^{\circ} \ldots \ldots \ldots \ldots \ldots . . . . . . .84$

70. Schematics of flow structrue for rotating conditions....................................... 86 


\section{LIST OF ABBREVIATIONS}

\begin{tabular}{|c|c|c|}
\hline PIV & ................. & Particle Image Velocimetry \\
\hline FFT & (1) & Fast Fourier Transformation \\
\hline POD & & Proper Orthogonal Decomposition \\
\hline CME & & Calibration Measurement Error \\
\hline CEE & & Calibration Equation Error \\
\hline $\mathrm{CDE}$ & & Calibration Drift Errors \\
\hline $\mathrm{AE}$ & & Approximation Error \\
\hline HFE & & High Frequency Error \\
\hline SRE & 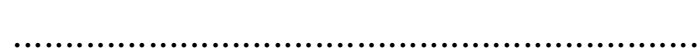 & Spatial Resolution Error \\
\hline
\end{tabular}




\section{LIST OF SYMBOLS}

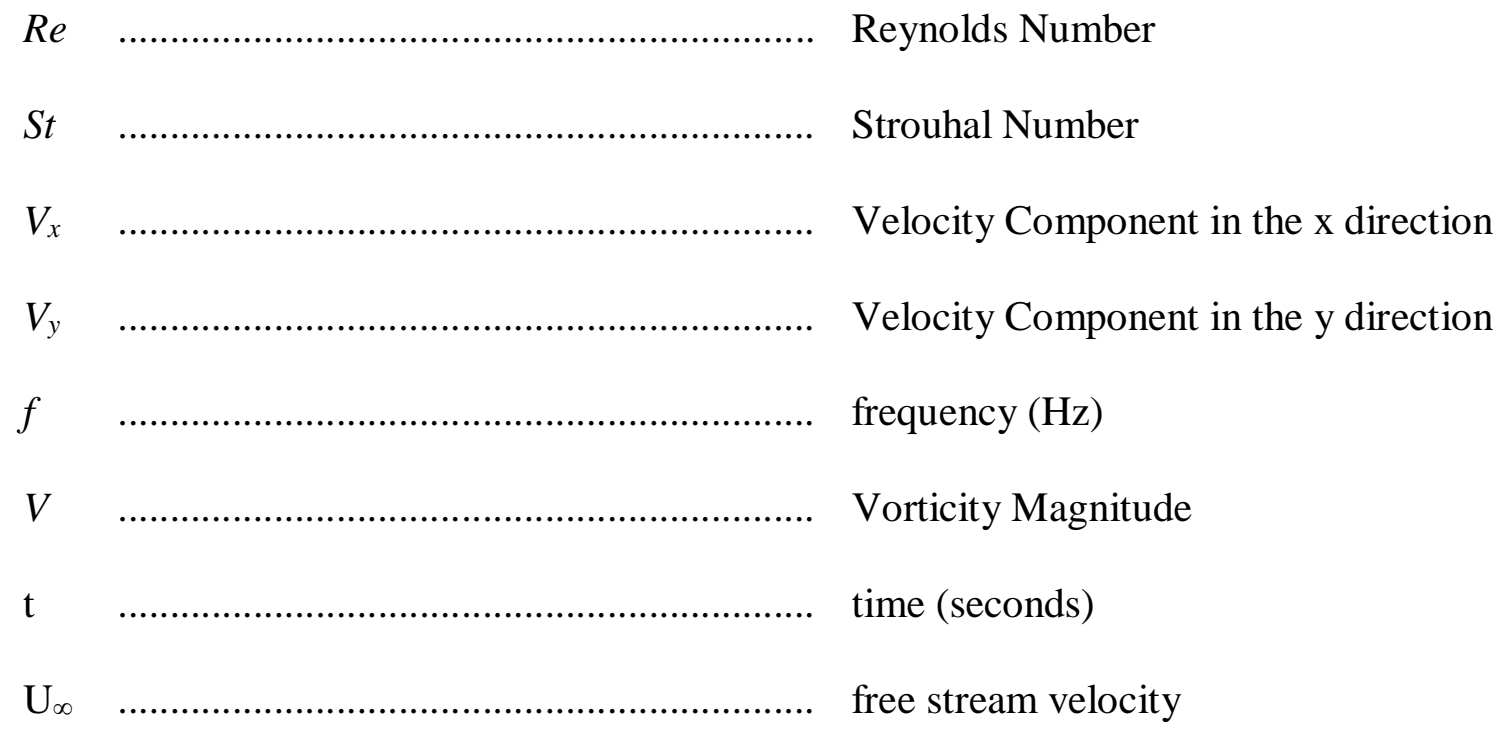




\section{LIST OF APPENDIX FIGURES}

$\underline{\text { Figures }}$

$\underline{\text { Page }}$

A.1. Single sided frequency spectrum at Reynold Number 57 and incident angle (a) $0^{\circ}$ (b) $45^{\circ}$ and (c) $180^{\circ}$

A.2. Single sided frequency spectrum at Reynold Number 500 and incident angle (a) $0^{0}$ (b) $45^{\circ}$ and (c) $180^{\circ}$

A.3. Single sided frequency spectrum at Reynold Number 858 and incident angle (a) $0^{0}$ (b) $45^{\circ}$ and (c) $180^{\circ}$

A.4. Single sided frequency spectrum at Reynold Number 1250 and incident angle (a) $0^{0}$ (b) $45^{\circ}$ and (c) $180^{\circ}$

A.5. Single sided frequency spectrum at Reynold Number 1700 and incident angle (a) $0^{0}$ (b) $45^{\circ}$ and (c) $180^{\circ}$ .96

A.6. Single sided frequency spectrum at Reynold Number 4350 and incident angle (a) $0^{0}$ (b) $45^{\circ}$ and (c) $180^{\circ}$

A.7. Single sided frequency spectrum at Reynold Number 7470 and incident angle (a) $0^{0}$ (b) $45^{\circ}$ and (c) $180^{\circ}$ .98

B.1. Single sided frequency spectrum at Reynold Number 57 and Rotation RPM (a) 120 (b) 360 (c) 960 (d) 1500 (e) 1800 (f) 2400 (g) 3000 RPM

B.2. Single sided frequency spectrum at Reynold Number 500 and Rotation RPM (a) 120 (b) 360 (c) 960 (d) 1500 (e) 1800 (f) 2400 (g) 3000 RPM 101

B.3. Single sided frequency spectrum at Reynold Number 858 and Rotation RPM (a) 120 (b) 360 (c) 960 (d) 1500 (e) 1800 (f) 2400 (g) 3000 RPM 103

B.4. Single sided frequency spectrum at Reynold Number 1250 and Rotation RPM (a) 120 (b) 360 (c) 960 (d) 1500 (e) 1800 (f) 2400 (g) 3000 RPM 105

B.5. Single sided frequency spectrum at Reynold Number 1700 and Rotation RPM (a) 120 (b) 360 (c) 960 (d) 1500 (e) 1800 (f) 2400 (g) 3000 RPM 107

B.6. Single sided frequency spectrum at Reynold Number 4350 and Rotation RPM (a) 120 (b) 360 (c) 960 (d) 1500 (e) 1800 (f) 2400 (g) 3000 RPM 109

B.7. Single sided frequency spectrum at Reynold Number 7470 and Rotation RPM (a) 120 (b) 360 (c) 960 (d) 1500 (e) 1800 (f) 2400 (g) 3000 RPM

D.1. Instantaneous Velocity Contour at $\mathrm{Re}=858$ and Rotation Speed is (a) 120 (b) 360 (c) 960 (d) 1500 (e) 1800 (f) 2400 (g) 3000 RPM 
E.1. Average Velocity Contour at $\mathrm{Re}=57$ and Rotation Speed is (a) 120 (b) 360

(c) 960 (d) 1500 (e) 1800 (f) 2400 (g) 3000 RPM.

E.2. Average Velocity Contour at $\mathrm{Re}=858$ and Rotation Speed is (a) 120 (b) 360

(c) 960 (d) 1500 (e) 1800 (f) 2400 (g) 3000 RPM

E.3. Average Velocity Contour at $\mathrm{Re}=1250$ and Rotation Speed is (a) 120 (b) 360

(c) 960 (d) 1500 (e) 1800 (f) 2400 (g) 3000 RPM

E.4. Average Velocity Contour at $\mathrm{Re}=1700$ and Rotation Speed is (a) 120 (b) 360

(c) 960 (d) 1500 (e) 1800 (f) 2400 (g) 3000 RPM

E.5. Instantaneous Velocity Contour at $\mathrm{Re}=1250$ and Rotation Speed is (a) 120 (b) 360

(c) 960 (d) 1500 (e) 1800 (f) 2400 (g) 3000 RPM

E.6. Instantaneous Velocity Contour at $\mathrm{Re}=1700$ and Rotation Speed is (a) 120 (b) 360

(c) 960 (d) 1500 (e) 1800 (f) 2400 (g) 3000 RPM

F.1. Standard deviation at $\mathrm{Re}=858$ and Rotation Speed is (a) 120 (b) 360 (c) 960

(d) 1500 (e) 1800 (f) 2400 (g) 3000 RPM

F.2. Standard deviation at $\mathrm{Re}=1250$ and Rotation Speed is (a) 120 (b) 360 (c) 960

(d) 1500 (e) 1800 (f) 2400 (g) 3000 RPM

G.1. Instantaneous vorticity plot when the cylinder orientation was (a) $0^{\circ}$ (b) $180^{\circ}$

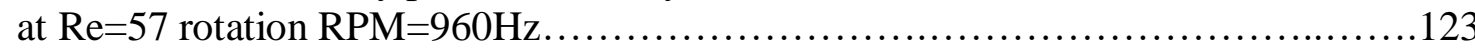

G.2. Instantaneous vorticity plot when the cylinder orientation was (a) $0^{0}$ (b) $180^{\circ}$ at $\mathrm{Re}=57$ rotation $\mathrm{RPM}=1500 \mathrm{~Hz}$.

G.3. Instantaneous vorticity plot when the cylinder orientation was (a) $0^{\circ}$ (b) $180^{\circ}$ at $\mathrm{Re}=57$ rotation $\mathrm{RPM}=1800 \mathrm{~Hz}$.

G.4. Instantaneous vorticity plot when the cylinder orientation was (a) $0^{\circ}$ (b) $180^{\circ}$ at $\mathrm{Re}=57$ rotation $\mathrm{RPM}=2400 \mathrm{~Hz}$.

G.5. Instantaneous vorticity plot when the cylinder orientation was (a) $0^{\circ}$ (b) $180^{\circ}$ at $\mathrm{Re}=57$ rotation $\mathrm{RPM}=3000 \mathrm{RPM}$.

H.1. Instantaneous Velocity Contour at $\mathrm{Re}=500$ and Rotation Speed is (a) 120

(b) 360 (c) 960 (d) 1500 (e) 1800 (f) 2400 (g) 3000 RPM . 128

H.2. Instantaneous Velocity Contour at $\mathrm{Re}=858$ and Rotation Speed is (a) 120

(b) 360 (c) 960 (d) 1500 (e) 1800 (f) 2400 (g) 3000 RPM

H.3. Instantaneous Velocity Contour at $\mathrm{Re}=1250$ and Rotation Speed is (a) 120

(b) 360 (c) 960 (d) 1500 (e) 1800 (f) 2400 (g) 3000 RPM 130 


\section{CHAPTER 1. INTRODUCTION}

\section{Purpose}

The study of flow around three-cylinder rotating system is a very important problem for both engineering application and fundamental research interest point of views. Flow over bluff bodies have many practical applications like flow in heat exchanger, propulsion, wind turbine, helicopter rotor, and controlling the vortex shedding to reduce the unsteady forces and vibration acting on an engineering structure. In addition to the existing application, a three-cylinder system will have many pioneering applications. For example, three-cylinder system can be used to generate a complex wake mixing system that will have potential application in turbulent mixing. Another practical interest is the placement of three-cylinder rotating system upstream of airfoils to create high intensity turbulent flow to analyze the performance of the airfoil under practical operating conditions. Although many studies have reported to understand the flow over single and multiple cylinders for different static position, no study has been found yet that considers the rotating of three cylinder to study the wake mixing. This study will help to reveal the flow structure past three-cylinder system at various Reynolds Number and rotation speed with the help of advanced diagnosis techniques such as 2D and 3D Particle Image Velocimetry complemented with hot wire measurements for time-resolved data series.

\section{Thesis Outline}

This thesis aims to provide an experimental evaluation of flow past three-cylinder rotating system. The following section, Chapter 2, provides background and history for the application of flow past rotating cylinder and understanding of wake mixing behavior from three cylinder. Chapter 3 discusses about the experimental setup and different parameter that has been used in hotwire, PIV, and Tomography setup. Then all the results obtained from the experiment are 
outlined in Chapter 4. In this chapter, all the instantaneous velocity, average velocity, vorticity, standard deviation maps are shown to discuss the flow characteristics. Chapter 5 discusses the advance proper orthogonal analysis. This section shows the modal decomposition and reconstruction of velocity based on POD. Lastly, Chapter 6 condenses all of the results from the previous chapters into a concise summary of the results and provides information regarding potential future work which can be done to continue this subject of study. Chapter 7 concludes with discussion of our findings. 


\section{CHAPTER 2. BACKGROUND}

The rotation of a three-cylinder rotating system can create high-intensity turbulent flow having significant consequences in separation and turbulence level on that airfoil for various AoA and Reynolds numbers. Such applications of the three-cylinder rotating system make it unique from previous studies that have been done on the flow around three-cylinder for a different equilateral arrangement with different spacing ratio $[1,2]$. These studies were done in order to reduce the flow-induced vibrations in engineering structures, fluid mixing, and acoustic noise generation. The near wake structure in three equidistant cylinders depends on the spacing to diameter ratio of each cylinder, the relative position of each cylinder [1, 3, 4]. Since this system rotates relative to the axis perpendicular to the flow, changes in RPM generate a variety of complex wake structures in the flow field. The use of three-cylinder rotation in the upstream of a dimpled airfoil has been reported in our previous study [5]. However, no study has comprehensively been published that shows the alteration of flow structure with various RPM of the cylinder and flow velocity using high-resolution PIV and tomography to reveal details of the complex wake interactions.

The study of flow past single rotating cylinder has been extensively studied [6-13]. A study by Prandtl already addressed that the maximum lift generated by a rotating cylinder is $4 \pi$. Both experimental and numerical studies $[7,14]$ have suggested that the Strouhal number increases with the rpm of a rotating cylinder. Taneda [15] reported flow visualization results of the bluff body wakes in various rotating motions. A review of some major observations has been published on flow past rotating cylinder at low Reynolds numbers [15]. Unlike flow past a single rotating cylinder, the flow past multiple cylinders is more complex. The flow interference of two cylinders

placed in tandem is reported by Hori [16] and King and Jones [16, 17]. Hori [16] found the 
difference of pressure coefficient of two equal cylinders when ' $1 / \mathrm{d}$ ' ratio are 1.2 and 2.0 where ' 1 ' is the spacing between two cylinders and $\mathrm{d}$ is the diameter of each cylinder. From the study done by Ishigai, 1972; Bearman and Wadcock, 1973; and Tatsuno, 1991 [18-20], it is observed that even though the two cylinders side by side are symmetrical to the oncoming flow, the flow field around the cylinders is not symmetrical. As a result, two cylinders experience different drag and lift forces due to the proximity interference when the spacing between the two cylinders is small. Like the study of flow interference past two cylinders in various arrangements, numerous papers have been published on the flow around three cylinders. Igarashi and Suzuki, 1984 [21] investigated the characteristics of the flow around three cylinders arranged in-line. They found three cases about the behavior of the shear layer of three cylinders- the first is a case without reattachment, the second with reattachment, and the third, rolling up in the front region of the downstream cylinders. The flow drag and lift forces, in the in-flow and cross-flow directions, have been evaluated for both downstream and upstream of two side-by-side cylinders from initially symmetrical to staggered arrangements [22]. For the case of three cylinders arranged in an equidistant triangular cluster, a smoke [4] and dye flow visualization [1,2] studies show different complex interactions of the vortex streets for various Reynolds number and spacing ratio at various incident angles. Experimental study done by Lam, $\mathrm{K}$ et. al shows how the strouhal number changes with varing incident angle of cylinder arrangement for range of spacing ration. The results shows sudden increase and decrease in Strouhal number when the spacing to diameter ratio of cylinder is very small. As the spacing ratio increases the Strouhal number approaches to 0.2 for each cylinders which is equal to single cylinder vortex shedding frequency. This result proves that with intermediate specing the wake interaction significantly affects vortex-shedding frequency. As the spacing ration increase, the wake interaction becomes less and each cylinder sheds individual 
vortex similar to single cylinder. Besides, numerical simulations of flow over three circular cylinders in equilateral arrangement [3] also show the interaction of wake from each of the cylinders at a different spacing ratio ranging from 0.5 to 4.0 and for three incidence ang les, $\alpha=0^{0}$, $30^{\circ}$, and $60^{\circ}$. They found that the flow around the three-cylinder system is divided into three stages according to the spacing. A small spacing when $s=0.5$, the proximal region dominates the flow pattern. At intermediate spacing, $0.5<\mathrm{s}<2.0$, vortex shedding occurs behind the downstream cylinders suppress the vortex shedding from upstream, which creates a complicated effect from the combination of proximity and shear layer interference. At $0^{\circ}$ and $180^{\circ}$ incident angle, the flow shows in-phase vortex shedding. At large spacing, for example, at $s=4$, a fully developed vortex shedding occurs behind all the cylinders. These flow phenomena also observed in the experimental study of smoke and dye flow visualization study [1], [2], [4].. 


\section{CHAPTER 3. EXPERIMENTAL SETUP}

\section{Three-Cylinder System Setup}

The main component of this study is the Three-cylinder setup, as shown in Figure 1. The cylinder is supported by a two-aluminum post at the end to prevent wobble motion. The threecylinder was arranged in an equidistant triangular arrangement. The diameter of each cylinder is $3.10 \mathrm{~mm}$, and the inner distance between each of the cylinders is $5.30 \mathrm{~mm}$. The spacing to diameter ratio of the cylinder is 1.70 . One post inside of the tunnel was installed close to the wall so that it does not have any effect on the flow. The cylinder is driven by a motor, and a power supply box powered the motor. The motor is capable of delivering 3000 RPM. To read the frequency, a circuit was built using the IR sensor.

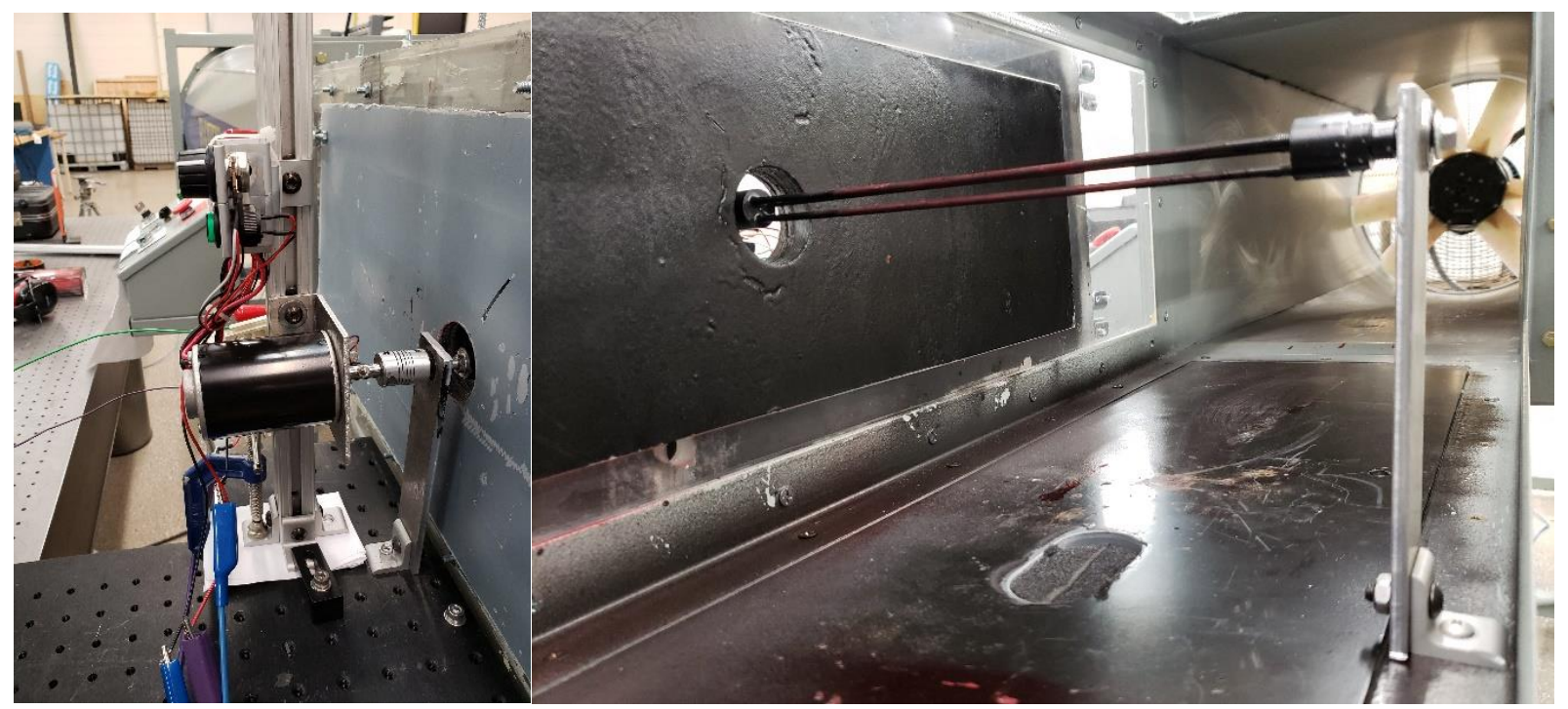

Figure 1. Three-cylinder Setup (a) installed in wind tunnel (b) Motor drive to rotate the threecylinder setup

\section{Wind Tunnel}

The data which will be collected for this investigation will be captured using equipment and methods currently used and practiced at NDSU. Any apparatus needed to actuate the airfoil will be constructed to replicate the rapid airfoil oscillation. NDSU is currently equipped with a 
FloTek 1440 wind tunnel which will be used to carry out these tests shown in Figure 2. The wind tunnel is capable of delivering a consistent free stream velocity of $U_{\infty}=36 \mathrm{~m} / \mathrm{s}$ and the throat has dimensions of $12 " \mathrm{x} 12$ ". This simple wind tunnel will be capable of recreating flow conditions in the computational experiments.

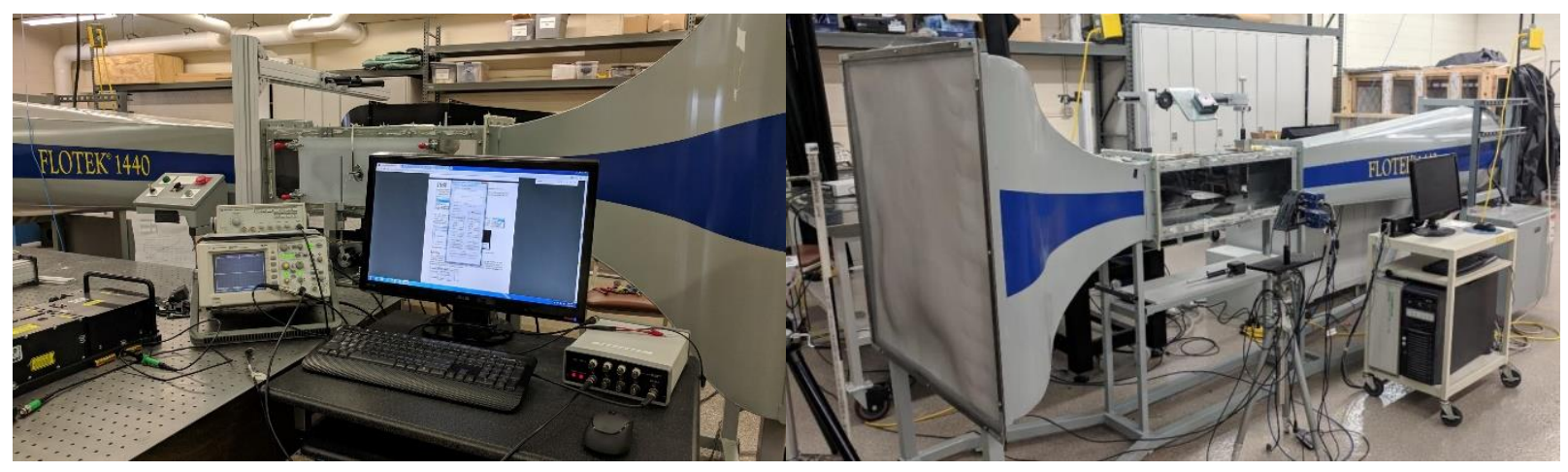

Figure 2. NDSU's FloTek 1440 wind tunnel and workstation

\section{Hotwire Setup}

Hotwire anemometry is one of the high frequency and cheapest flow measurement technique that allows the velocity fluctuation in a fixed position in the flow field [23]. The hotwire used in this experiment was constant temperature hotwire. The how wire was made of very thin tungsten wire. This wire is connected to the one arm of the Wheatstone bridge. The electrical current is used in order to heat the wire. By the use of servo amplifier, the bridge remains in balance with the passage of variable current in order to keep the resistance of the wire hence the temperature of wire remains constant. A DAQ was used to get the voltage measurement that was required to keep the wire temperature constant as data format.

To do the calibration at first, a hotwire was placed along with a pitot tube side by side to correlate the voltage with the flow velocity as shown in Figure 3 (a). We considered a third order polynomial that provided a good fit between the data and the function. Figure 3 (b) shows the third order polynomial function fit through all the data points. 
After calibration, the hotwire was placed at three different positions. In first stage, the hotwire was kept very close to the cylinder as shown in Figure 4 (a). When the hotwire was positioned very close, data was taken at two different locations. One was in the middle of the cylinder system and another set of data was taken close to the top edge of the three-cylinder system setup. In the second stage, data was taken with hot wire placed far from the three-cylinder system but within the 10 diameter distance so that to capture the vorticity information near field which is shown in Figure 4 (b). Data was taken at two different locations when the hot wire was placed very close to three cylinder because the vorticity from each cylinder system will be different at different transverse location. However, when the hotwire was placed far from three-cylinder setup then the transverse variation of vorticity is much phase locked and one single location was considered sufficient.

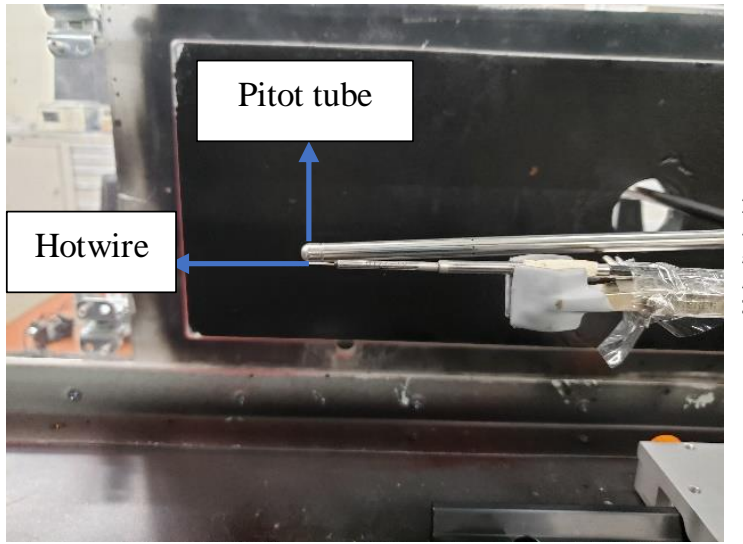

(a)

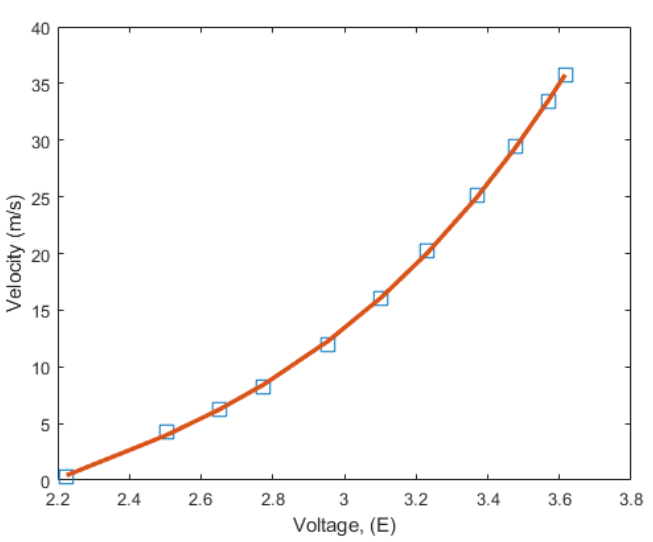

(b)

Figure 3. Hotwire calibration setup using Pitot tube (a) Calibration between voltage and velocity (b). 


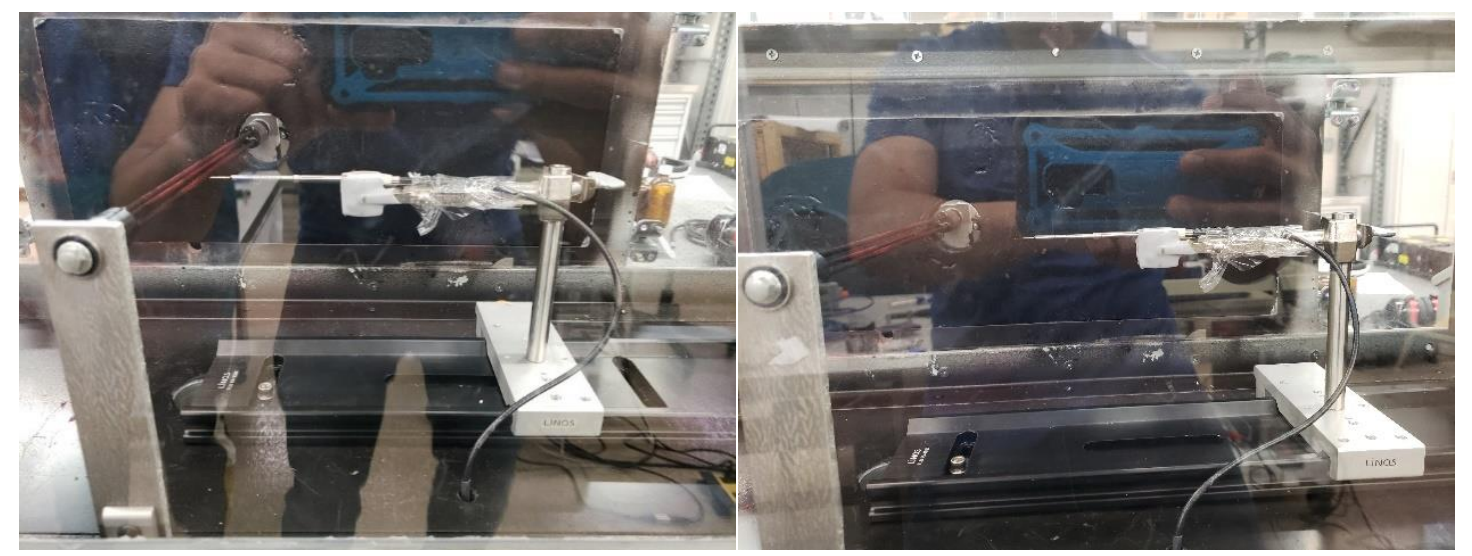

Figure 4. Hotwire positioned very close to the three cylinder system (a) far from the cylinder system (b)

\section{Particle Image Velocimetry (PIV) Setup}

For the PIV system, the schematic of the system used in the present study is shown in Figure 3 and photography of the four-camera PIV setup and of the laser sheet on three rotating cylinder system in the wind tunnel is shown in Figure 5.

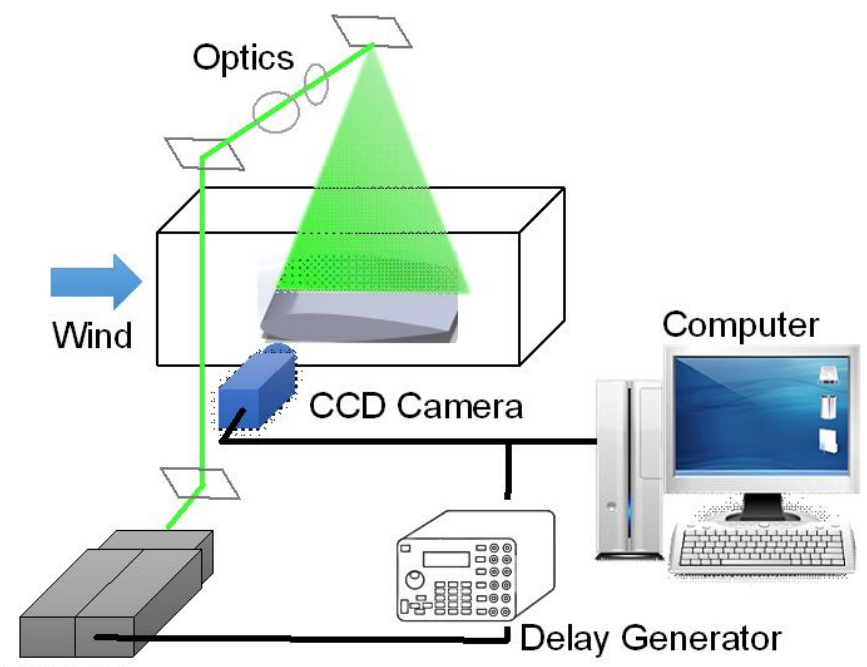

Nd:YAG Laser

Figure 5. Schematic of Standard PIV system 
The test cylinder system was installed in the middle of the test section. Illumination was provided by a double-pulsed Nd:YAG laser (NewWave MiniLase-III) capable of emitting two laser pulses of $100 \mathrm{~mJ}$ at a wavelength of $532 \mathrm{~nm}$ with a repetition rate of $15 \mathrm{~Hz}$. The laser beam was shaped to a laser sheet (thickness $<1 \mathrm{~mm}$ ) by using a set of mirrors, spherical and cylindrical lenses. The flow was seeded with DEHS (Diethylhexyl Sebacate) particles using Laskin nozzle atomizer system. A high resolution CCD PIV camera with resolution of $\sim 1600 \mathrm{x} 1200$ pixels and inter frame capability of $<1 \mathrm{~ms}$ was set up with its axis perpendicular to the laser sheet for image acquisition. The CCD camera and the Nd:YAG lasers were connected to a PTU unit from LaVision which control the timing of the LASER illumination and the image acquisition. This system installed system advance flow measurement lab is shown in the Figure 6.

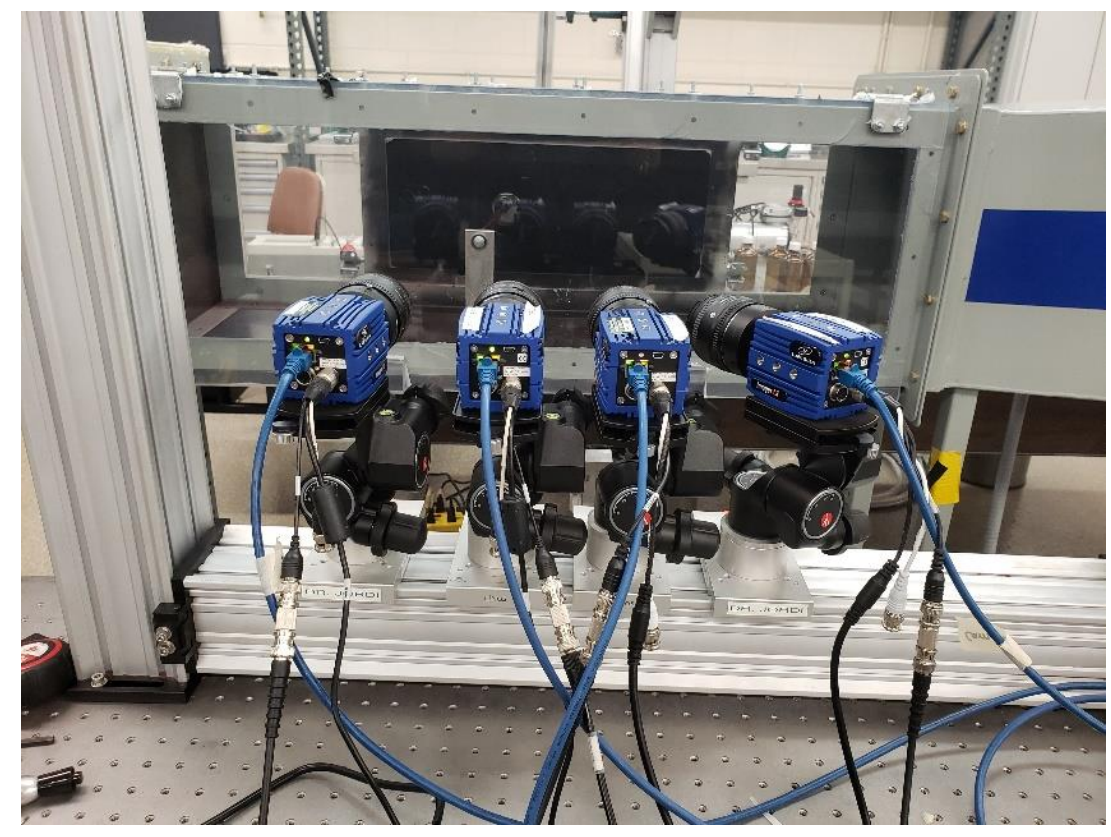

Figure 6. Four-camera PIV setup in a wind tunnel

Instantaneous PIV velocity vectors were obtained by a frame to frame cross-correlation technique involving successive frames of patterns of particle images in multi-pass interrogation process from window size of 128x128 to 32x32 pixels. An effective overlap of $75 \%$ was employed. The time delay between the two frames was set to $100 \mathrm{~ms}$ to $10 \mathrm{~ms}$ depending on the air speed to 
deliver adequate displacements within the flow velocity dynamic range. The time-averaged quantities were obtained from a cinema sequence of 100 frames of instantaneous velocity fields for each case. Note that the instantaneous velocity plots are time unresolved due to low speed LASER.

The seeding of the air during testing was acquired by dispersing atomized 'DEHS' oil into the inlet corresponding only to the location of the laser volume being projected. Before collecting the data, scaling was done by LaVision ' $\mathrm{DaV}$ is 10 ' software. In order to remove the area that did not have the LASER illumination and to provide a clean reconstruction of the airfoil visualized by the data set, different mask was applied to the raw image.

To minimize the laser reflection from the surface cylinder, we used flat-black paint and "Dykeem Steel Red" paint on the surface of the cylinder. The red paint absorbed the green laser color and only a dim red reflection remained, which could readily filtered out in the image with a camera green filter.

In PIV campaign we captured data in two stage. At first the cameras were placed so that each of the camera obtain $80 \mathrm{~mm}$ view. Therefore, two cameras were able to give as data equal to $160 \mathrm{~mm}$ data downstream. In the second stage, we brought the camera very close to cylinder system so that each of the camera obtain data from $40 \mathrm{~mm}$ physical field of view. So, two camera was able to see $80 \mathrm{~mm}$ view in downstream. This was done to see the wake structure in more detail. The calibration comparison from in two camera is shown in the Figure 7. 

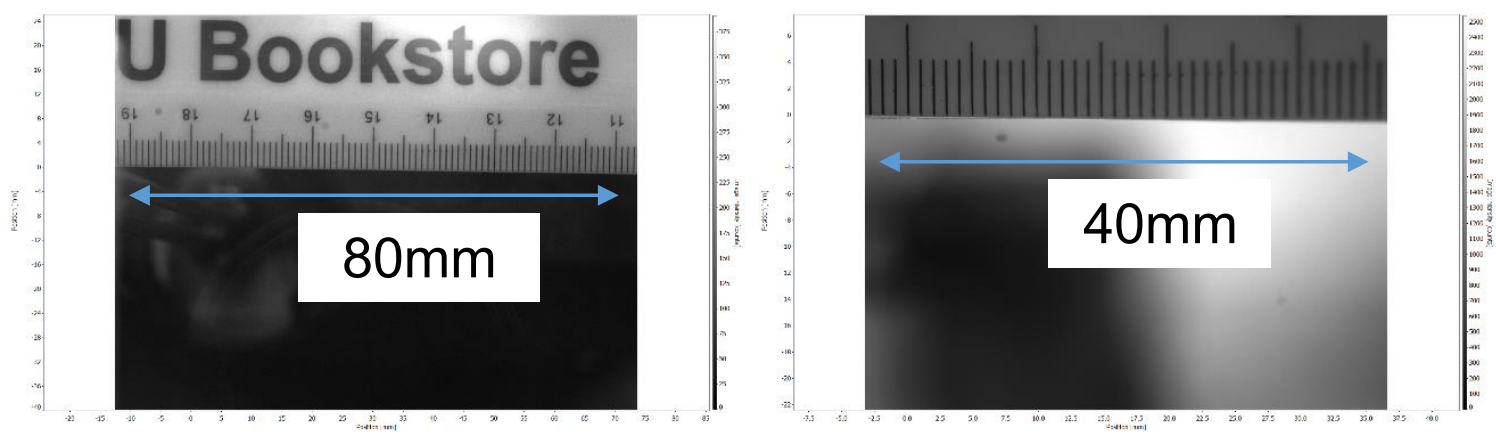

Figure 7. Calibration comparison (a) Far view (b) close view

\section{Tomography Setup}

In order to obtain the three dimensional data of the flow around a three-cylinder rotating system, 3D tomography measurement was applied. In our tomography setup, we used four LaVision Imager LX camera as shown in Figure 8 (a). The laser system consisted of the same dual head laser used in 2D PIV (NewWave MiniLase-III Nd:YAG) and a combination of PIV cameras and adjustable Scheimpflug adapters from La Vision. The camera and LASER were synchronized with a PTU unit. For tomographic PIV, a camera volume calibration was needed. A dual plane, dual sided calibration target for 3D measurements with dimensions 204 x 204 mm from LaVision (model 106-10) was used for the calibration as shown in Figure 8 (b). The calibration, data acquisition, image processing, and data processing (volume self-calibration, 3D reconstruction, MART algorithm, etc.) was performed with the Flow Master DaVis 10 (LaVision) software supporting four cameras.

In order to record data for a volume, the LASER sheet was widened to about $6 \mathrm{~mm}$ as shown in Figure 9 with the use of a cylindrical lens and a aperture before projected into the top of three-cylinder. The aperture makes the LASER sheet sharp at the edges and improves the data quality. To allow accurate reconstruction, the 4 cameras were placed on one side of the wind tunnel. Figure 8 (a) displays the location of each camera just behind the three cylinder. After aligning the laser and cameras for ideal volume reconstruction, the cameras were calibrated using 
LaVision's calibration plate as shown in Figure 8 (b). The plate contained two surfaces to allow for 3-dimensional calibration with calibration dots of $2.5 \mathrm{~mm}$ in diameter and $10 \mathrm{~mm}$ distance vertically and horizontally from other calibration dots. All four cameras were calibrated at the same time with the center of the two calibration planes in the middle of projected laser volume. After that the software proceeds to locate the calibration dots for each camera and configure the volume reconstruction steps.

The seeding of the air during testing was acquired by atomized 'DEHS' oil. We have used two sets of nozzle to seed the particle in the air so that the seeded particle cover the whole LASER illumination. The data was taken by computer controlling four camera and the LASER power. After collecting data, all the raw image was evaluated and based on the image quality we have applied several filter in the image preprocessing operation for different test condition and image quality on each test condition. The maximum number of particles that were used to calculate the peak density was 100,000 . The volume was reconstructed using the max $\mathrm{x}$ and $\mathrm{y}$ ranges and a $\mathrm{z}$ range of -3 to $3 \mathrm{~mm}$. The allowed triangulation error that was calculated and used was 1.5 pixels. The calibration was set to create $5 \mathrm{x}$ and $\mathrm{y}$ sub-volumes with $3 \mathrm{z}$ sub-volumes. To correct for outlining calibration vectors, vector processing was performed on the self-calibration before applying the new calibration. The default "Universal outlier detection/removal/insertion" setting was used along with smoothing $3 \times 3 \times 3$ at a multiplication of $1 \mathrm{x}$ and a strength of 0.5 . To ensure the entire image was corrected, all the sub-volumes were filled up. To ensure the new calibration did not need to be corrected again, self-calibration was performed again. Before applying the calibration, the new calibration vectors were found to be insignificant in magnitude and were aligned in the same direction. The volume reconstruction was set to construct the volume with the same settings used in the self-calibration by applying the max $\mathrm{x}$ and $\mathrm{y}$ dimensions and with the $\mathrm{z}$ 
dimension of -3 to $3 \mathrm{~mm}$. The volume correlation used 4 correlation window sizes starting from a voxel size of 96 to 48 with each step using an overlap percentage of $75 \%$. The peak search radius and volume binning was set to start at 8 . Neither of the correlation volume or multi-pass postprocessing sub-settings were used. The vector postprocessing 3D settings were set to the same used for the volume self-calibration. However, in this case, not all the voxels were filled up. Using the average velocity magnitude setting in the vector statistics process accommodated for the lack of completely filled planes from the previous process. This allowed for a clean reconstruction of the air flow visualized by the entire data set. Masking was applied to the areas without laser illumination.

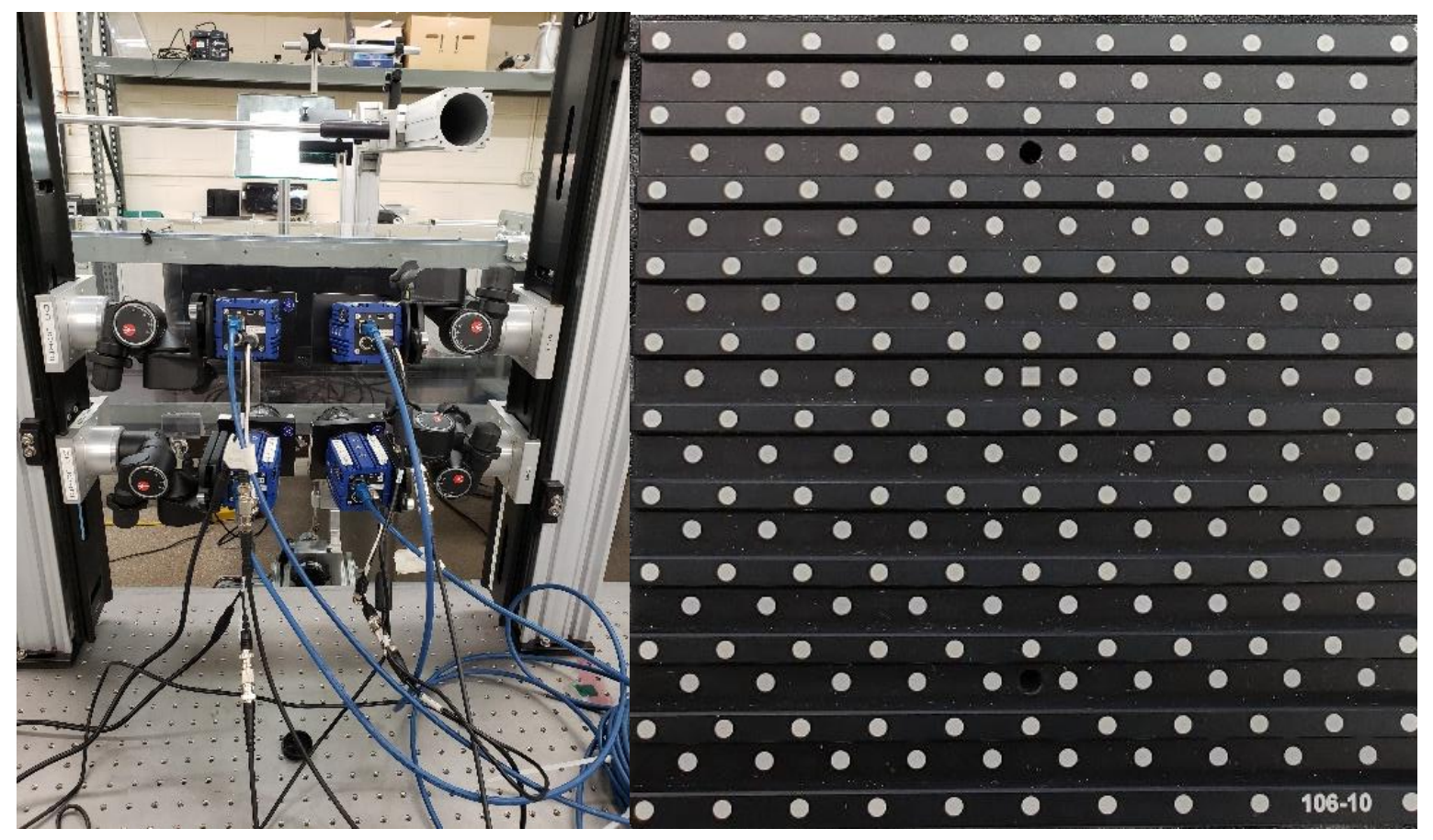

Figure 8. (a) Four-camera setup (b) 3D calibration plate 


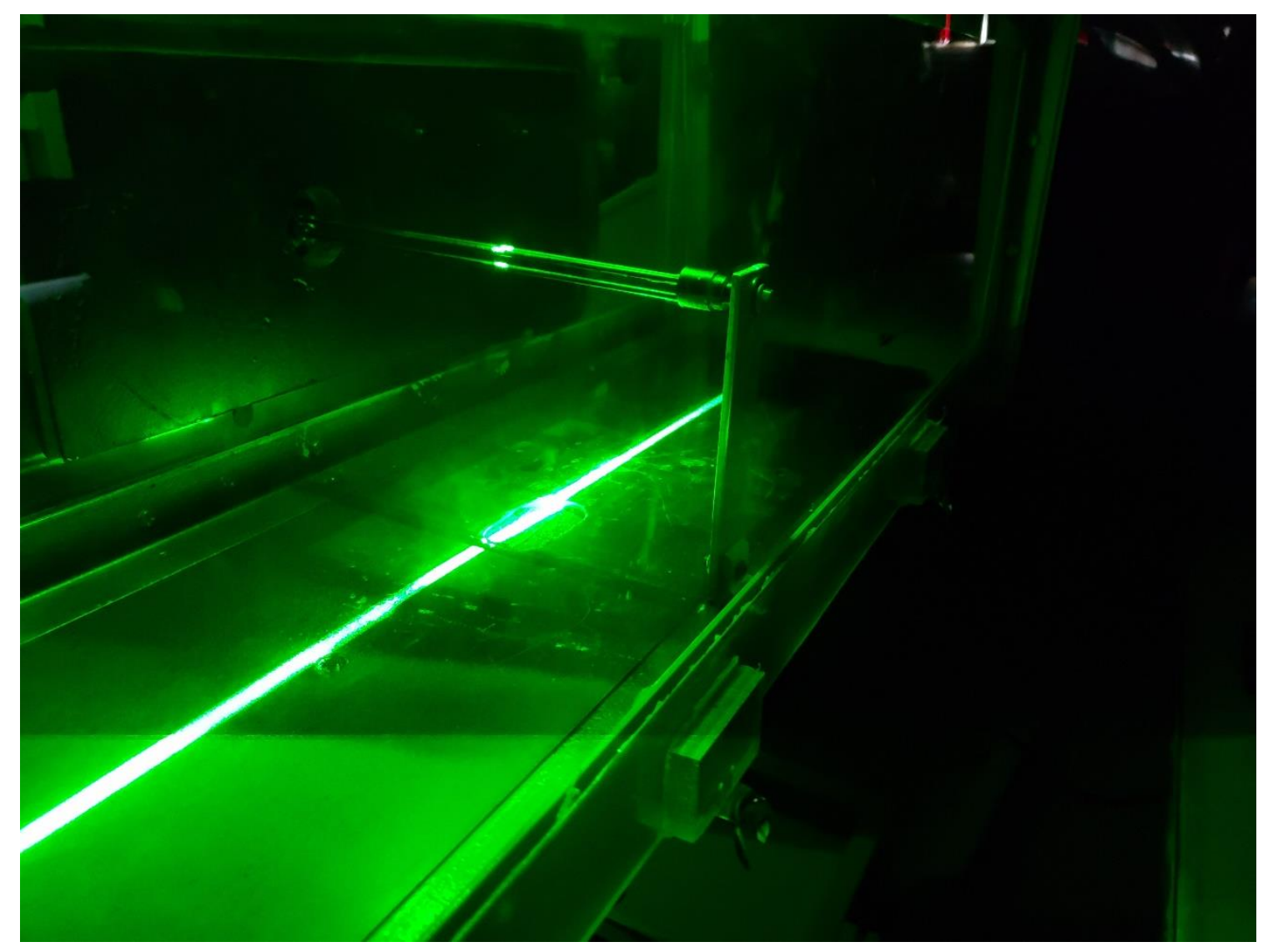

Figure 9. LASER illumination during Tomography Testing 


\section{CHAPTER 4. RESULTS AND DISCUSSION}

\section{Hotwire Data}

In the first step of our experiment, the velocity fluctuation with time was captured by hotwire probe for three different downstream position. The schematic of the position of hotwire probe is shown in the following Figure 10. The coordinate of the position a, b, and c is $(1.61,0)$, $(1.61,2.21)$, and $(32.2,0)$ respectively. The coordinates are given in terms of non-dimensional form which is done with respect to diameter of individual cylinder. The time series data obtained from the hotwire was converted to frequency spectrum based on Fast Fourier Transformation (FFT). All the frequency spectrum plot for different Reynolds number at both static and various different RPM is presented in APPENDIX A and APPENDIX B.

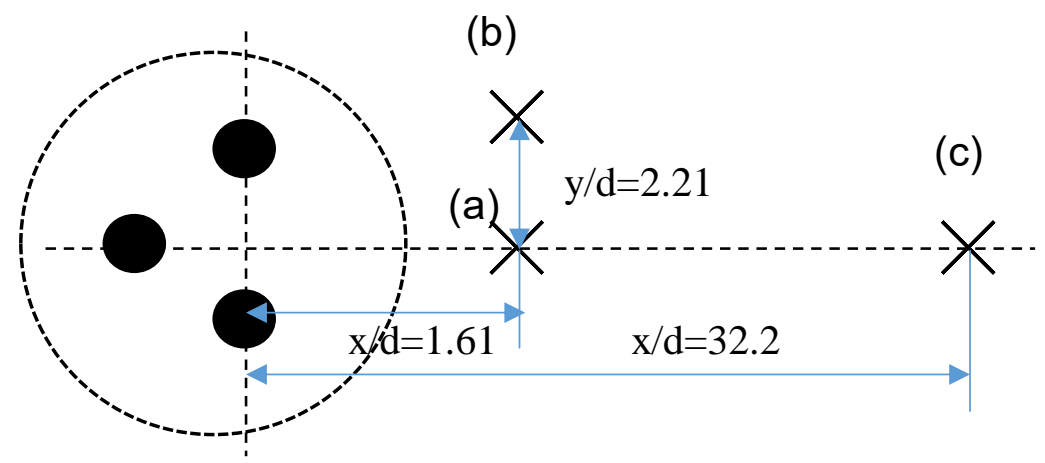

(a) \& (b) $-5 \mathrm{~mm} ; \quad$ (c) $-100 \mathrm{~mm}$

Figure 10. Schematic positions of hotwires to obtain the time resolved data 


\section{$\underline{\text { Non-Rotating Cases }}$}

At static conditions, in APPENDIX A shows the frequency spectrum for three different orientations. The frequency spectrum indicates the absence of distinct peaks for many conditions. However, when the hotwire probe was placed at location (a), the distinct peaks in the frequency spectrum are observed at $180^{\circ}$ incident angle orientation. Also, when the hotwire was placed at location (b), the distinct peaks are observed for $45^{\circ}$ incident angle orientation. From the frequency spectrum for those two instances, the Strouhal numbers are plotted as a function of Reynolds number that is shown in Figure 11. The diameter of each of the cylinders $(D=3.1 \mathrm{~mm})$ was taken as characteristic length to define the Strouhal number. From the plot, it shows that the vortex shedding frequency for various Reynolds numbers is very fluctuating in nature for two different orientation. When the Reynolds number is ranged between 500 to 1700, the Strouhal number varies between 0.1 to 0.3 , which is very different from the vortex shedding frequency of singlecylinder. This fluctuation of vortex shedding phenomena might occur due to the interaction of wake from three individual cylinders. For the rest of the cases, when the hotwire probe was at the location (a) and (b), the Strouhal number summary is shown in Table 01. At location (c), no vortex shedding was captured.

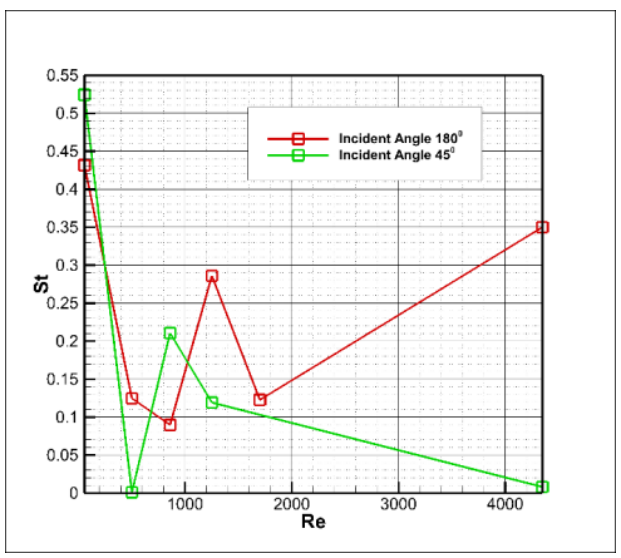

Figure 11. Strouhal Number plot with varying Reynolds number at Incident Angle $180^{\circ}$ and $45^{\circ}$ when the hotwire was placed at $\mathrm{x} / \mathrm{d}=1.61, \mathrm{y} / \mathrm{d}=2.21$, and $\mathrm{x} / \mathrm{d}=1.61, \mathrm{y} / \mathrm{d}=0$ respectively 
Table 1. Summary of Stouhal number for different orientation of cylinder system at Position (a) and (b)

\begin{tabular}{|c|c|c|c|c|c|c|}
\hline & \multicolumn{3}{|c|}{ position-(b) } & \multicolumn{3}{c|}{ Position-(a) } \\
\hline Reynolds Number & $0^{0}$ & $45^{0}$ & $180^{0}$ & $0^{0}$ & $45^{0}$ & $180^{0}$ \\
\hline $\mathbf{5 7}$ & - & 0.416533 & 0.431506 & - & 0.524149 & - \\
\hline $\mathbf{5 0 0}$ & - & 0.129346 & 0.124584 & - & 0.000988 & - \\
\hline $\mathbf{8 5 8}$ & - & 0.17758 & 0.089651 & - & 0.210542 & - \\
\hline $\mathbf{1 2 5 0}$ & - & - & 0.285973 & - & 0.119071 & 0.002583 \\
\hline $\mathbf{1 7 0 0}$ & 0.002023 & - & 0.122784 & - & - & 0.111535 \\
\hline $\mathbf{4 3 5 0}$ & $4.72 \mathrm{E}-06$ & $4.72 \mathrm{E}-06$ & 0.34985 & 0.13866 & 0.00805 & 0.098705 \\
\hline $\mathbf{7 4 7 0}$ & - & - & - & $2.75 \mathrm{E}-06$ & 0.008561 & 0.063336 \\
\hline
\end{tabular}

\section{Rotating Cases}

When the cylinder system rotates at various RPM, the hotwire probe was able to capture the frequency of vortex shedding at three different positions. The rotation of the three-cylinder system creates a complex mixing of wake; therefore, the vortex shedding frequency exhibits different characteristics than a single cylinder. In APPENDIX B shows the plot of vortex shedding frequency from time-series data by FFT calculation. In the frequency domain, the hotwire probe captures multiple vortex shedding frequencies. The existence of multiple peaks in the frequency domain is observed in most of the cases, mostly when the hotwire probe was located very close to the cylinder system. After observing the frequency of multiple peaks, it is found that they are multiple of each other. Also, some of the peaks have direct relation to RPM in the frequency domain. The highest peak is considered to represent the actual frequency. Other peaks are thought to be the frequency of vortices passing by the hotwire. The constant rotation of cylinder system shed multiple vortices that are in harmony to each other. From the frequency spectrum plot, the highest peak has been selected in order to find the relation between the Strouhal number (St) and RPM for various Reynolds numbers. The characteristic length to define the Strouhal number is considered as the diameter of the circle that escribe to the triangular arrangement of the cylinder 
arrangement. The Strouhal Number (St) relation as a function of RPM is shown in Figure 12. At very low $\mathrm{Re}=57$, the Strouhal number for each location of the hotwire probe exhibits very high value compared to other Re number. Since the St number shows different characteristics at $\operatorname{Re}=57$ then higher Re number that's why they were plotted in a separate graph for three position in Figure 12(a). At Re=57, Figure 12(a) shows the Strouhal number variation with RPM of cylinder system at three locations of the hotwire probe. The results indicate that the Strouhal number does not follow any particular trend as the RPM increases. At low RPM, the St value varies 0.3 to 0.45 . As the RPM increases, the St value has a sharp increase and decrease. This fluctuation might happen due to the complex vortex shedding because of mixing of the wake from three individual cylinder and their rotation. The mixing of wake from intermediate cylinder spacing could generate small but high-frequency vortices. The St value appears to reach very high (Approximately 1.9) when the hotwire probe was located at position (b). The St curve at the location (a) and (c) has a similar pattern of variation with RPM.

Figure 12 (b-d) shows the relation between Strouhal number (St) and RPM for different Reynolds number (Re) when the hotwire probe was placed at position (a), (b), and (c) respectively. From the comparison of results in Figure $12(\mathrm{~b}-\mathrm{d})$ indicate vortex shedding frequency from cylinder system varies with the position of hotwire probe in the flow domain. However, the results have a similar trend when the hotwire probe was paced close to the cylinder system (position a and b) then the results obtained in position c. These characteristics are indicative of much more turbulent and highly random vorticity field.

When the hotwire probe was placed at position-(a), the results in Figure 12 (b) indicates that the St value increases as the RPM of the cylinder system increase for different Reynolds (Re) number. However, at $\mathrm{Re}=500$, when the RPM approaches 960 and 1500, the St value has a sudden 
increase and decrease. Later, the St Value approaches to 0.2 for maximum RPM 3000.. As Re increases to 858,1250 , and 1700 , the St value increases very steeply with RPM. At maximum RPM, the St value reach close to 0.2 for $\mathrm{Re}=500,1250$, and $1700 . \mathrm{Re}=858$ has considerably high St value compare to $\mathrm{Re}=1250$ and 1700 . As the Re number increases further, for example, $\mathrm{Re}=4350$ and 7470, the St value decreases compare to low Re numbers. At $\mathrm{Re}=4350$ and 7470, St value increases linearly with RPM.

When the hotwire probe was placed at location-(b), the Strouhal number (St) has the same characteristics as Figure 12 (b) except for $\mathrm{Re}=500$ and 858. At $\mathrm{Re}=500$ and 858, the St value fluctuates to RPM ranged from 1500 to 2500 then reaches to the same value as before for maximum RPM.

At the position-(c), the hotwire probe captures the vortex shedding frequency at far downstream compare to position (a) and (b). The St value at this location has a different value than the other two locations. The St value is comparatively lower when the hotwire was placed at far downstream. At this point, most of the curve shows a sudden decrease of St value at maximum RPM. The fluctuation of St value with RPM at far downstream might be happed due to the complex mixing of positive and negative vortices. As the vortices travel far downstream, they become very unsteady in nature, and their vortex shedding frequency becomes lower compare to the upstream position. 


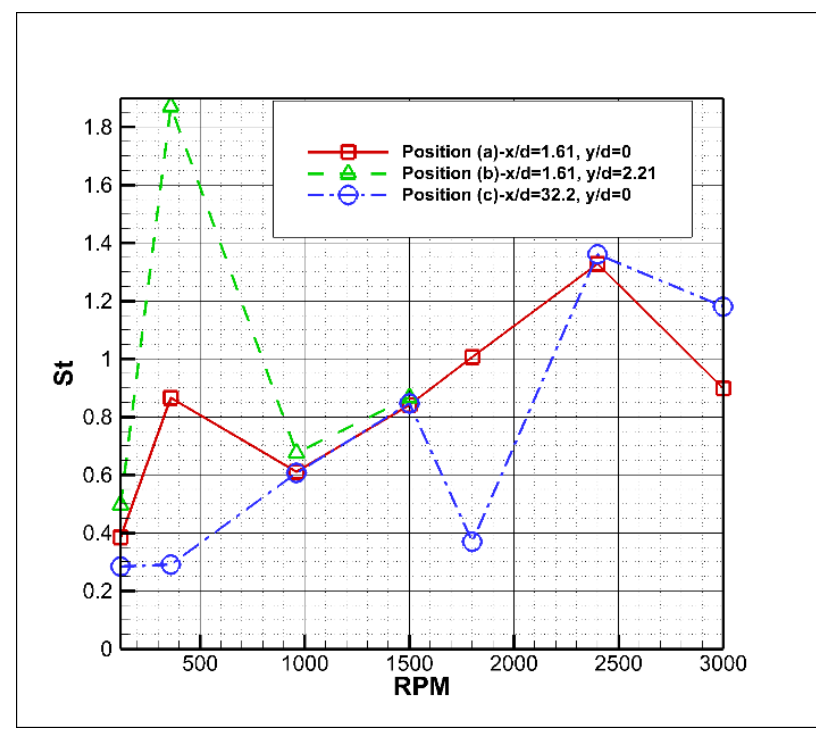

(a)

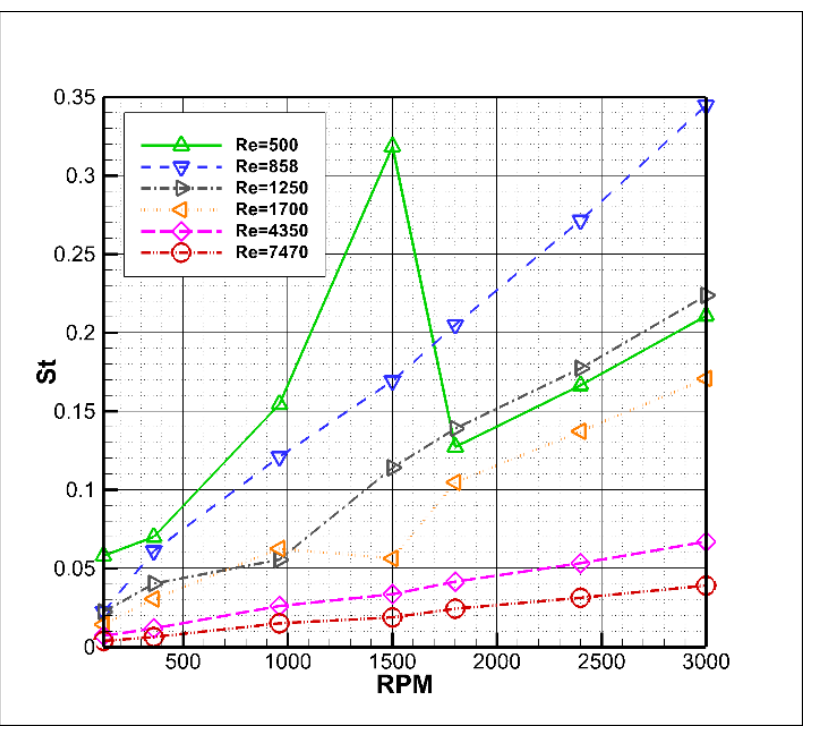

(b)

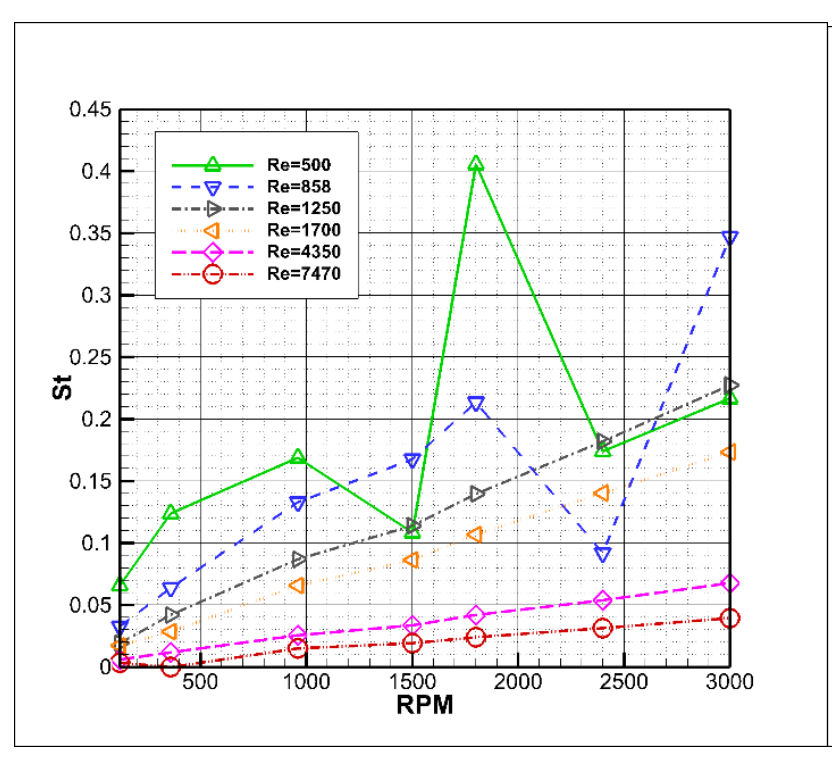

(c)

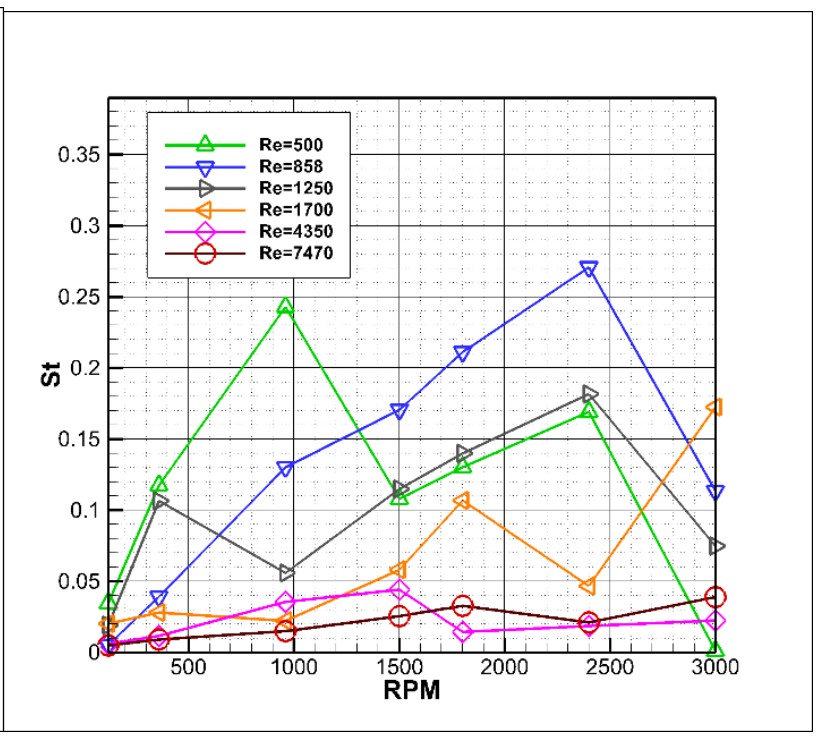

(d)

Figure 12. Strouhal Number plot as function of rotation for

(a) $\mathrm{Re}=57$ for three different location of hotwire probe,

(b) different Reynolds number when the hotwire probe was located at position-a,

(c) when the hotwire was located at position-b,

(d) when the hotwire was located at position-c 


\section{Particle Image Velocimetry (Far View)}

\section{$\underline{\text { Non-Rotating Cases }}$}

In this section, we have collected data using 2D Particle Image Velocimetry. Figure to Figure 16 shows the instantaneous vorticity contour for three different orientations of the static cylinder system when the Reynolds number varies from 57 to 1250 . The wake region in downstream and upstream has a distinct difference. The combination of wake from the individual cylinders make the wake cluster in the upstream complicated. Figure shows three uncorrelated time instances of instantaneous vorticity. The results indicate that the flow structures in upstream (close to the cylinder system) exhibits the mixing of positive and negative vortices due to the intermediate spacing of the cylinders. After the mixing, the vortices evolve to organized and symmetric vortex shedding in the downstream. Figure (a) shows vorticity magnitude $w=400$ at $(25,0)$ and $w=-400$ at $(20,-10)$ indicates the strong vorticity and symmetry of vortex shedding in this configuration. At $45^{0}$ orientation, due to the asymmetric orientation of the cylinder system, the wake mixing evolves to unorganized vortex shedding. Another feature that can be observed at a low Re number is that the downstream cylinder wake dominates the in the flow domain compare to the upstream cylinders. Due to the intermediate spacing, the wake from the upstream cylinder gets narrower that can be observed in Figure 13 (c). Figure (a-c) exhibits vortex shedding formation with spinning ring vortex, spiraling arms and mushroom structure. Red box marked in the figure are used to show the feature discussed. The features indicate typical shear layer formation and shows excellent quality of data and resolution, typically can be found in computation fluid dynamics data. As the Reynolds number increases, Figure 14 to Figure 16 shows that the mixing of positive and negative vortices becomes strong, and high turbulence creates a chaotic wake in the downstream for every orientation of the cylinder system. In addition, Figure to Figure 16 
reveals that with increasing Re number the vorticity strength increases. At high Re (Figure 16) number the vorticity stay strong for long distance compare to low Re number (Figure) which vorticity vanishes much more in upstream. These samples shown in Figure to Figure 16 shows good correlation of vortex diameter. As the Re number increases the vortex diameter reduces. Some downstream vortex is peaked in order to show a correlation between diameter of vortex and Re number in Figure 17. This suggests that high Re has smaller eddies compare to the larger vortices of low Re.

Figure 18 to Figure 22 shows the standard deviation map calculated based on 100 instantaneous data. Standard deviation measures the fluctuation of velocity in the flow domain. All the plots are shown on the same scale. The results exhibit a slight variation of turbulent intensity due to different orientations of the cylinder system. As the Reynolds number increases, the turbulent intensity in the upstream becomes larger.

Figure 23 - Figure 27 show the average stream wise velocity profile for three different cylinder orientation when $\operatorname{Re}=57,500,858,1250$, and 1700. All the plots were made on four different position. The $\mathrm{x}$-coordinate of the velocity profile were made non-dimensional with respect to single cylinder diameter. The non-dimensional $\mathrm{x}$ co-ordinate to diameter ratios are $\mathrm{x} / \mathrm{d}=0, \mathrm{x} / \mathrm{d}=12.9, \mathrm{x} / \mathrm{d}=25.8$, and 38.7. At $\mathrm{Re}=57$, the average stream wise velocity varies with the change of cylinder orientation. When the cylinder orientation is $0^{0}$, the velocity deficit at $\mathrm{x} / \mathrm{d}=0$ shows two peaks in the wake region. These peaks indicates the wake coming out from two downstream cylinder. As the orientation changes to $45^{\circ}$, the velocity deficit peaks changes but the profile are similar to $0^{0}$ orientation. As the cylinder orientation changes to $180^{\circ}$ the velocity profile looks very symmetric. The velocity profile indicates that the downstream cylinder wake dominates. The wake from the upstream cylinder gets weaken due to the presence of downstream 
cylinder. As the position moves to further downstream the velocity profile starts to become similar for three different cylinder orientation. As the Re number increases the velocity profile for $0^{0}$ and $45^{0}$ orientation shows similar trend. However, for $180^{\circ}$ orientation the wakes profile of upstream cylinder pairs starts to disappear in the profile with increasing Re and the wake from downstream cylinder starts to dominate more. With increasing Re number, the velocity profile at downstream locations $\mathrm{x} / \mathrm{d}=12.9, \mathrm{x} / \mathrm{d}=25.8$, and 38.7 become similar. This indicates the wake effect from individual cylinders mostly disappear in downstream. Their effect only exist close the cylinder arrangement in the upstream. 

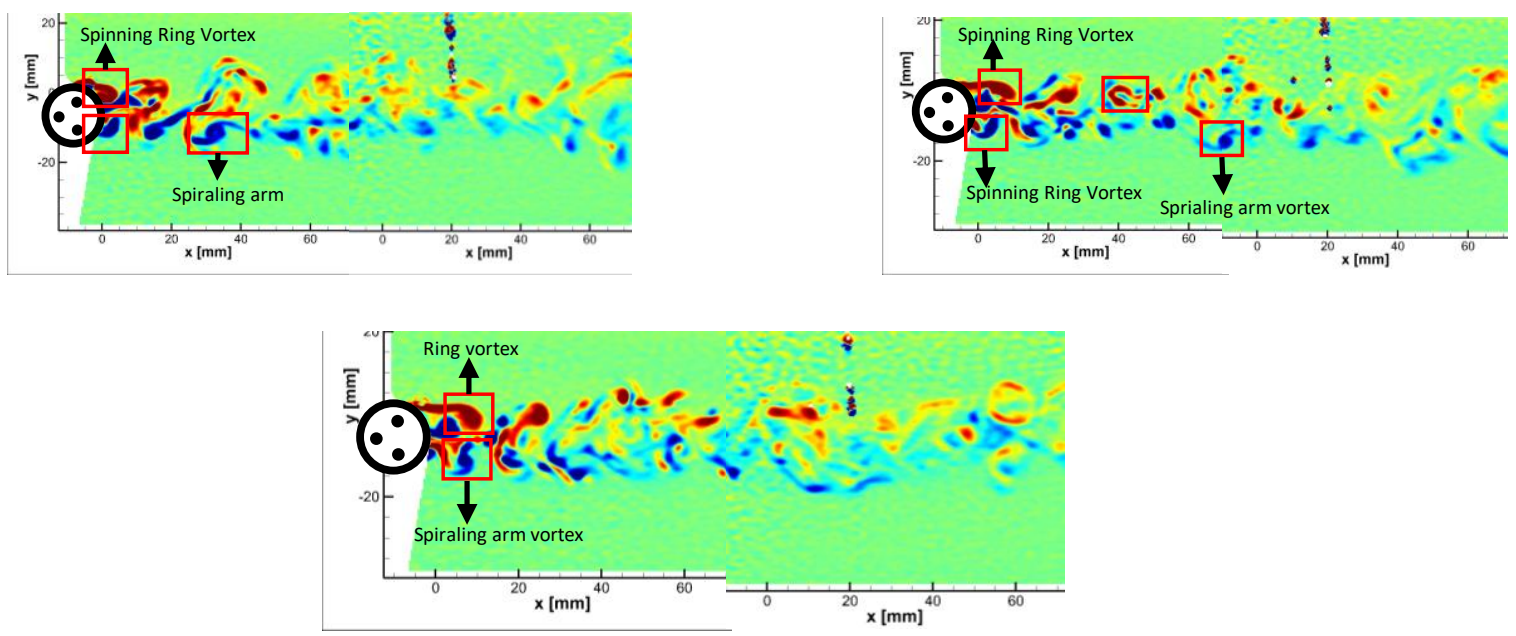

(a)
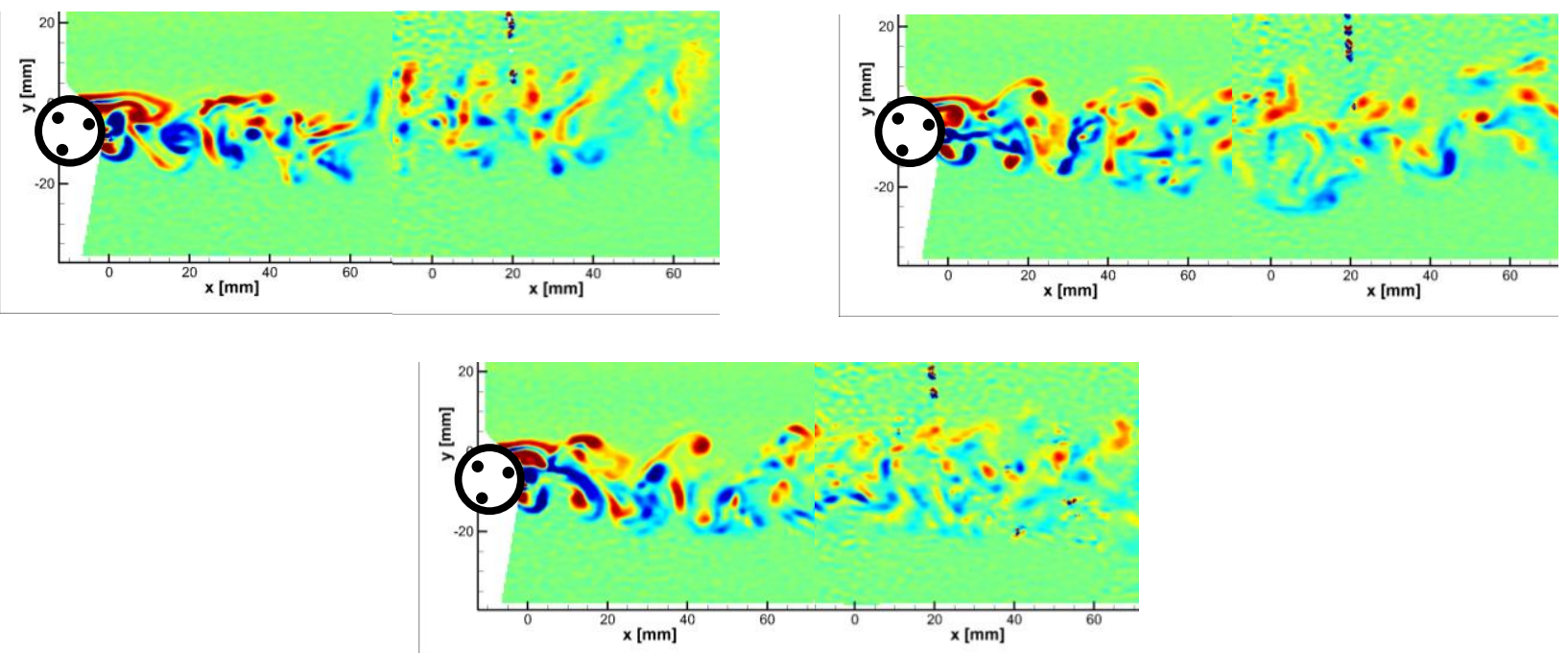

(b)

Figure 13. Instantaneous Vorticity contour at $\mathrm{Re}=57$ and Incident Angle (a) $0^{\circ}$, (b) $45^{\circ}$ and (c) $180^{\circ}$ 

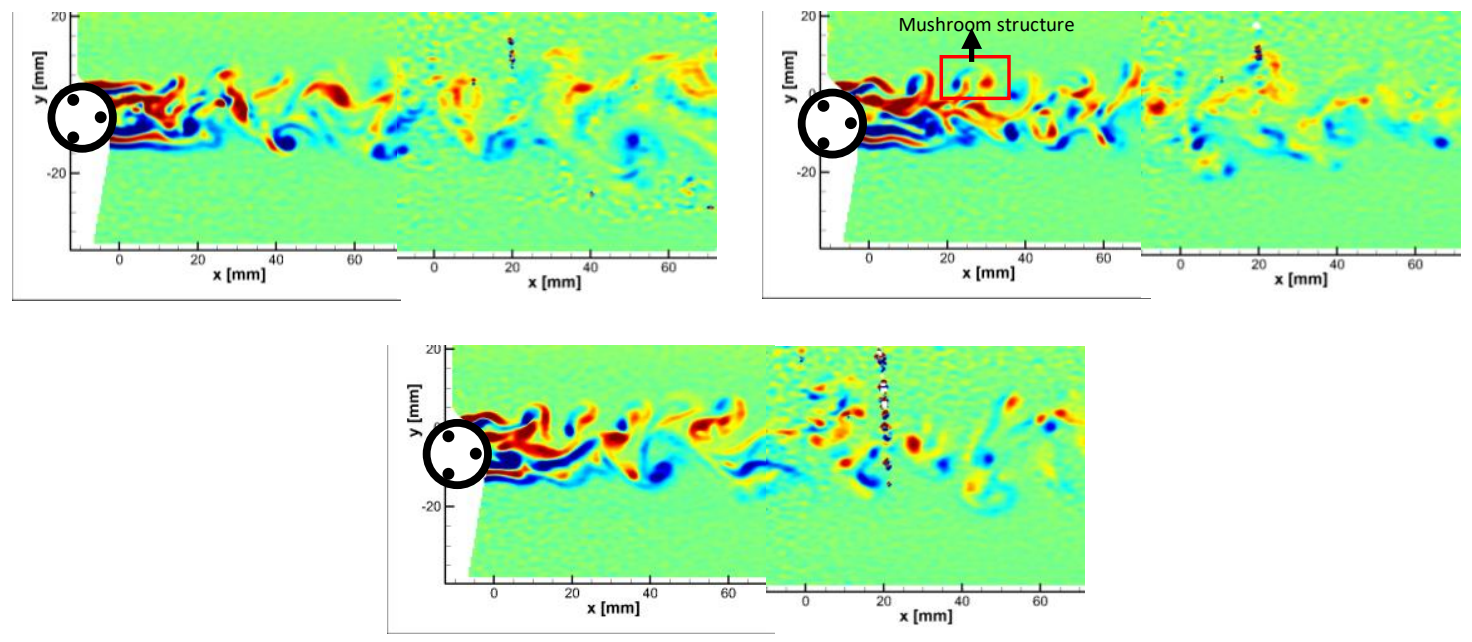

(c)

Figure 13. Instantaneous Vorticity contour at $\mathrm{Re}=57$ and Incident Angle (a) $0^{\circ}$, (b) $45^{\circ}$ and (c) $180^{\circ}$ (Continued)

vorticity [1/s]: $-500-400-300-200-100 \quad 0 \quad 100 \quad 200 \quad 300 \quad 400 \quad 500$

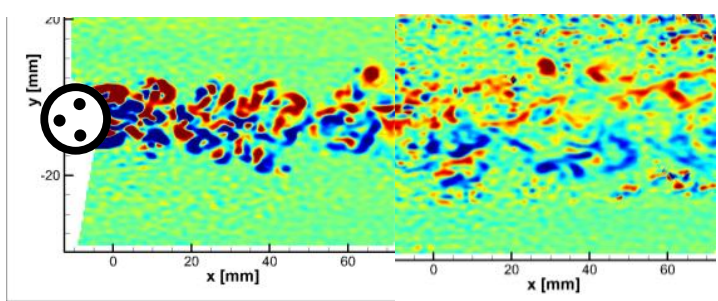

(a)

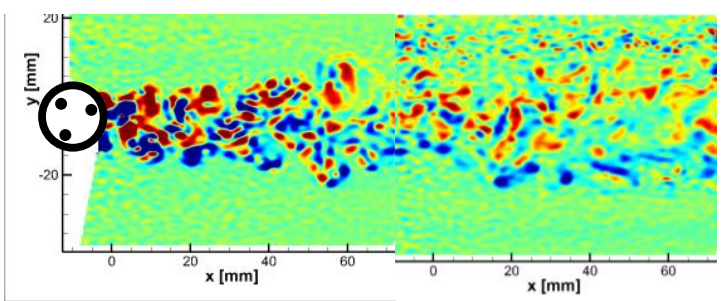

(b)

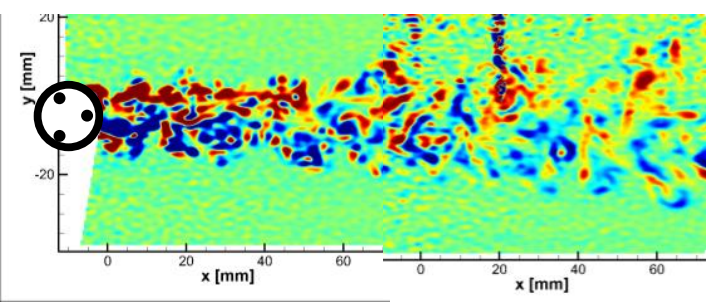

(c)

Figure 14. Instantaneous Vorticity contour at $\mathrm{Re}=500$ and Incident Angle (a) $0^{\circ}$, (b) $45^{\circ}$, and (c) $180^{\circ}$ 


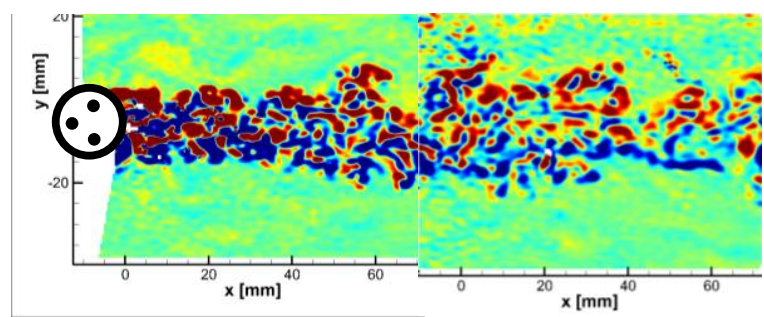

(a)

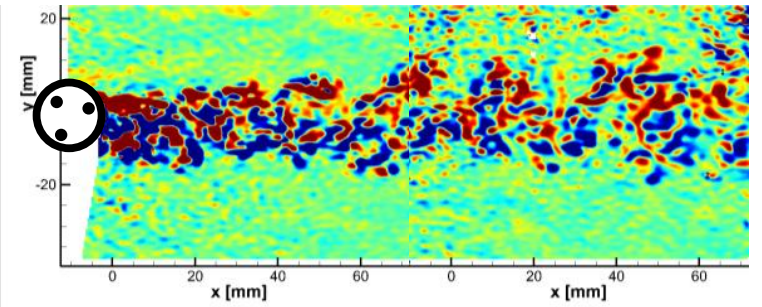

(b)

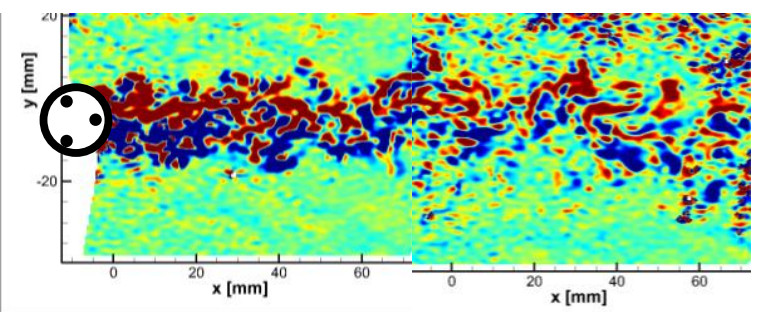

(c)

Figure 15. Instantaneous Vorticity contour at $\mathrm{Re}=858$ and Incident Angle (a) $0^{\circ}$, (b) $45^{\circ}$, and (c) $180^{0}$

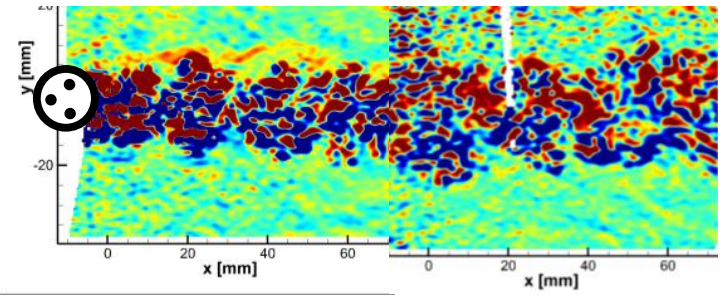

(a)

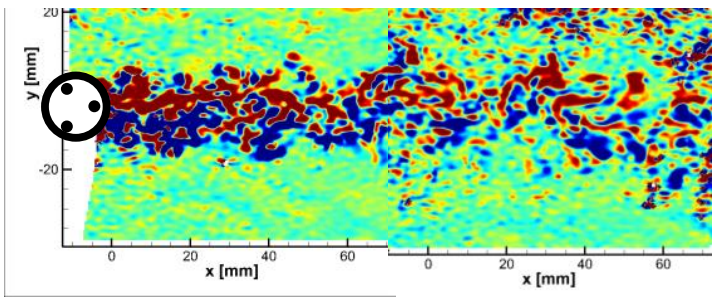

(b)

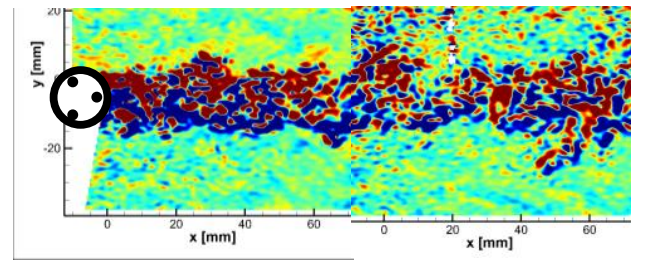

(c)

Figure 16. Instantaneous Vorticity contour at $\mathrm{Re}=1250$ and Incident Angle (a) $0^{\circ}$, (b) $45^{\circ}$, and (c) $180^{\circ}$ 


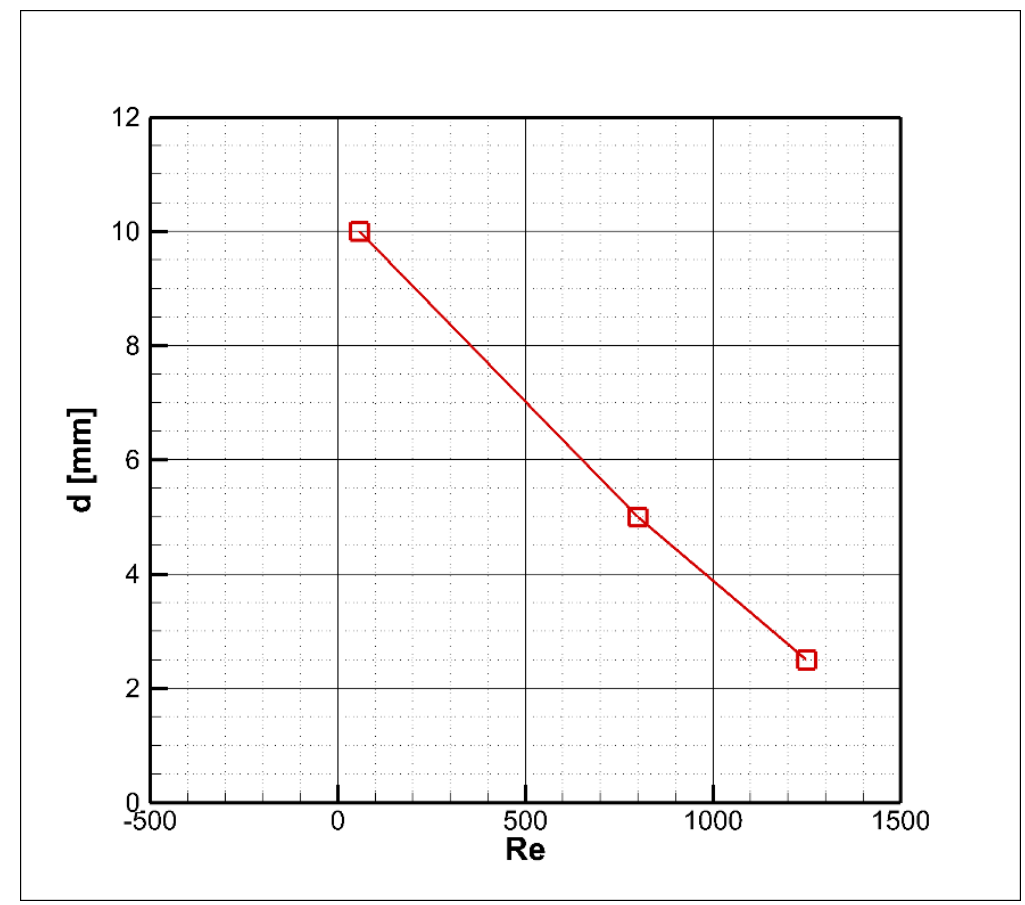

Figure 17. Variation of vorticity diameter with respect to Re number

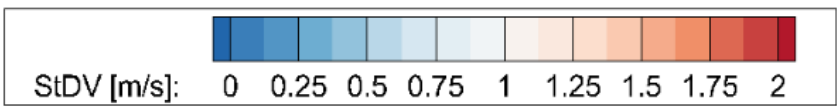

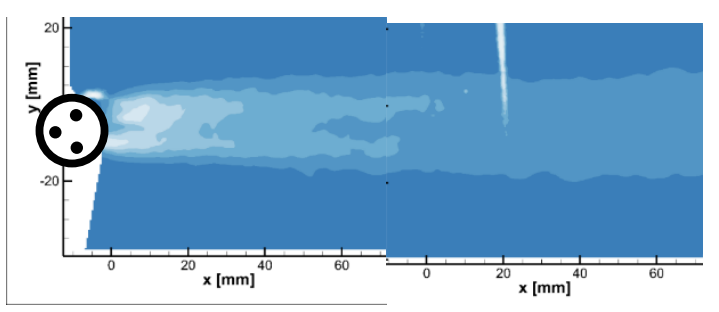

(a)

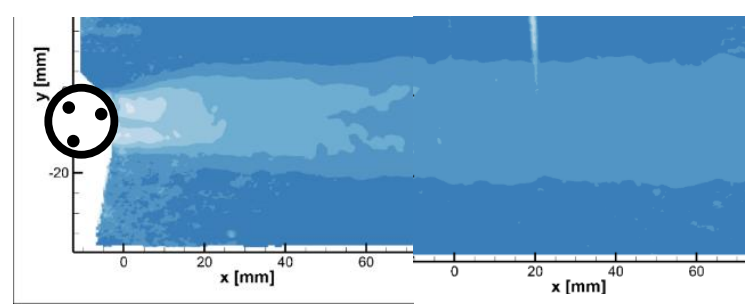

(b)

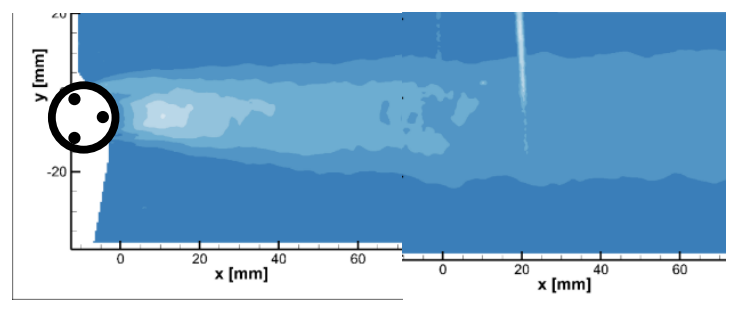

(c)

Figure 18. Standard Deviation contour at $\mathrm{Re}=57$ and Incident Angle (a) $0^{\circ}$, (b) $45^{\circ}$, and (c) $180^{\circ}$ 


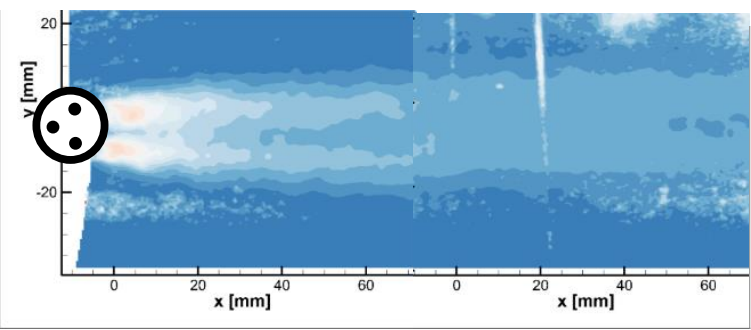

(a)

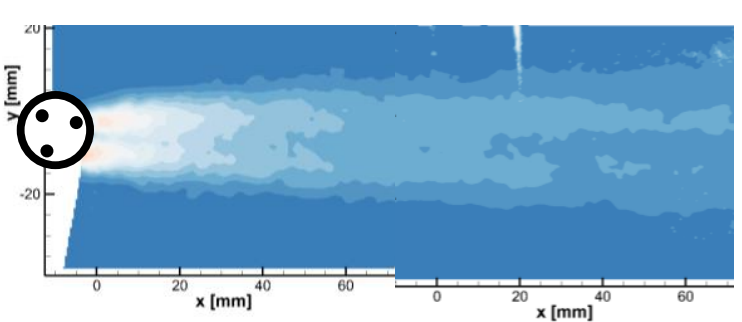

(b)

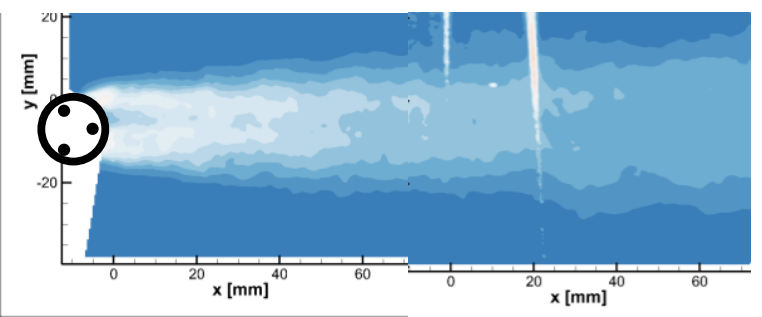

(c)

Figure 19. Standard Deviation at Re $=500$ and Incident Angle (a) $0^{\circ}$, (b) $45^{\circ}$, and (c) $180^{\circ}$

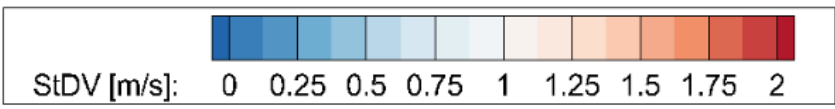

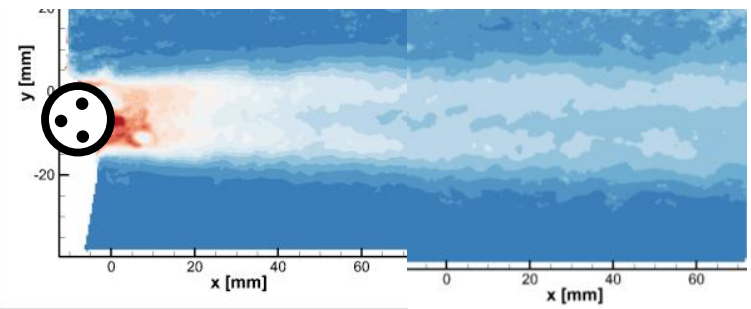

(a)

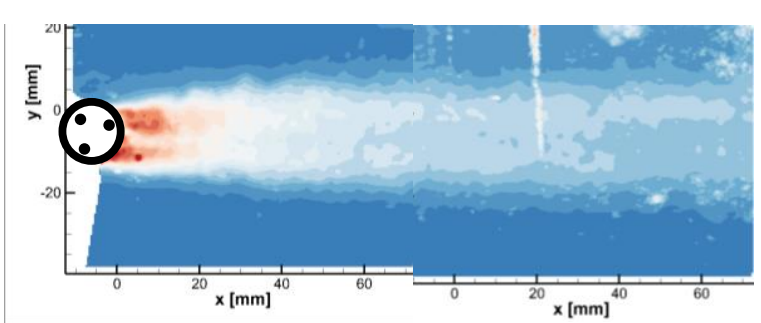

(b)

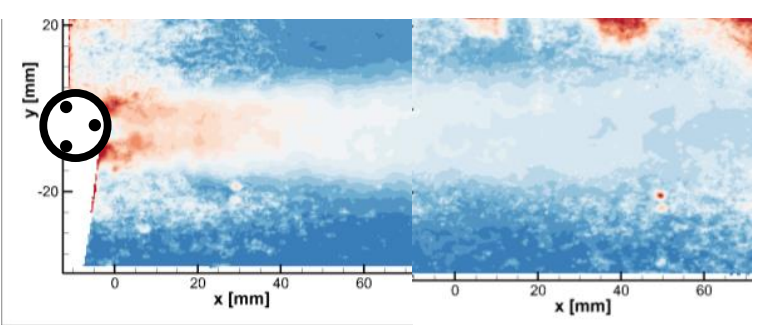

(c)

Figure 20. Standard Deviation at $\mathrm{Re}=858$ and Incident Angle (a) $0^{\circ}$, (b) $45^{\circ}$, and (c) $180^{\circ}$ 


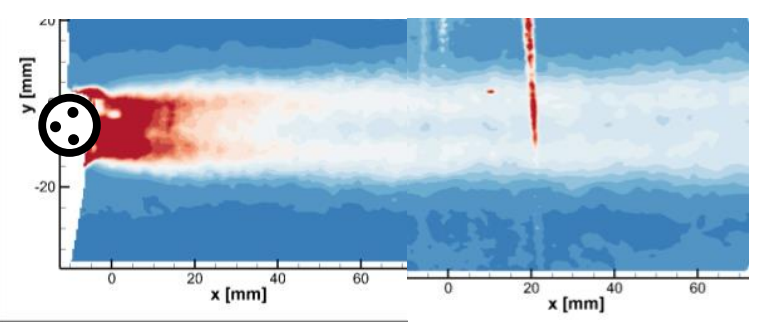

(a)

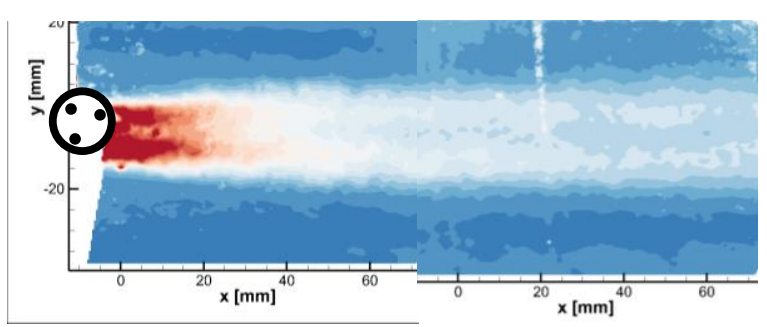

(b)

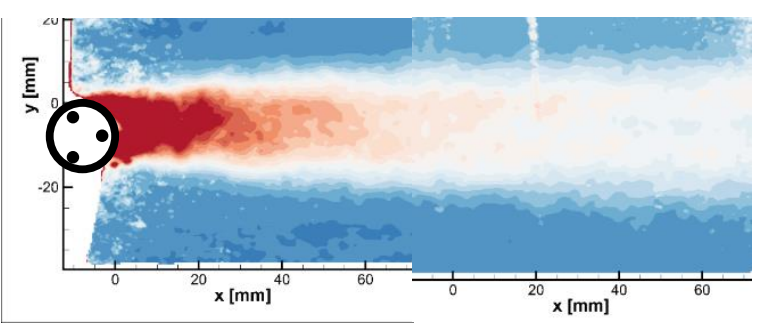

(c)

Figure 21. Standard Deviation at $\mathrm{Re}=1250$ and Incident Angle (a) $0^{\circ}$, (b) $45^{\circ}$, and (c) $180^{\circ}$

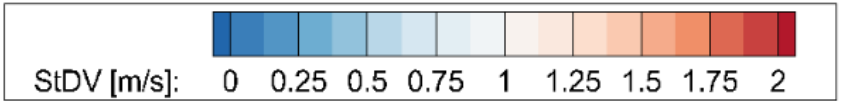

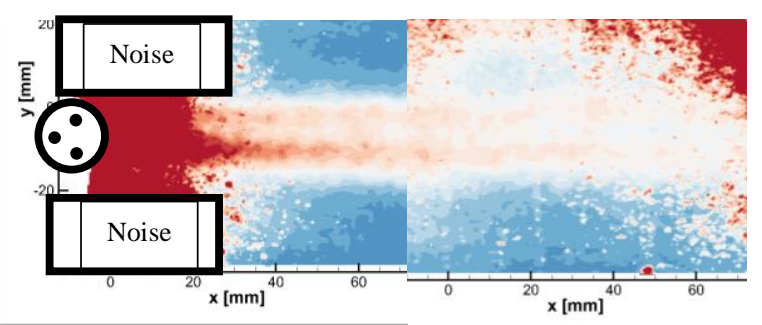

(a)

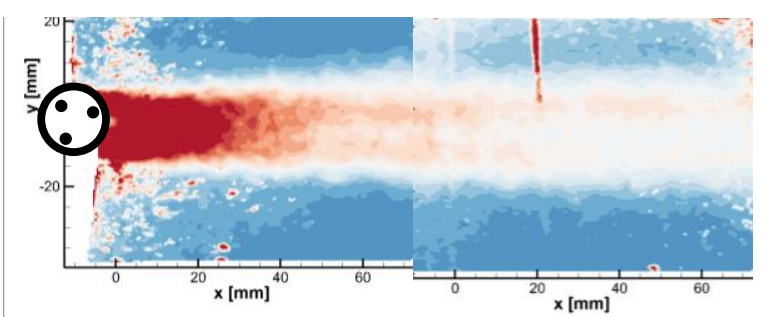

(b)

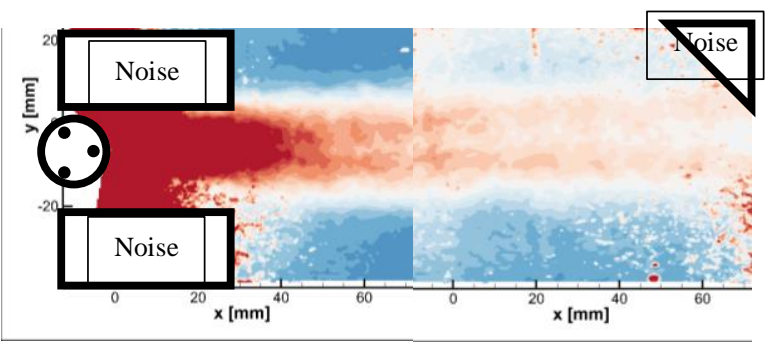

(c)

Figure 22. Standard Deviation at $\operatorname{Re}=1700$ and Incident Angle (a) $0^{\circ}$, (b) $45^{\circ}$, and (c) $180^{\circ}$ 


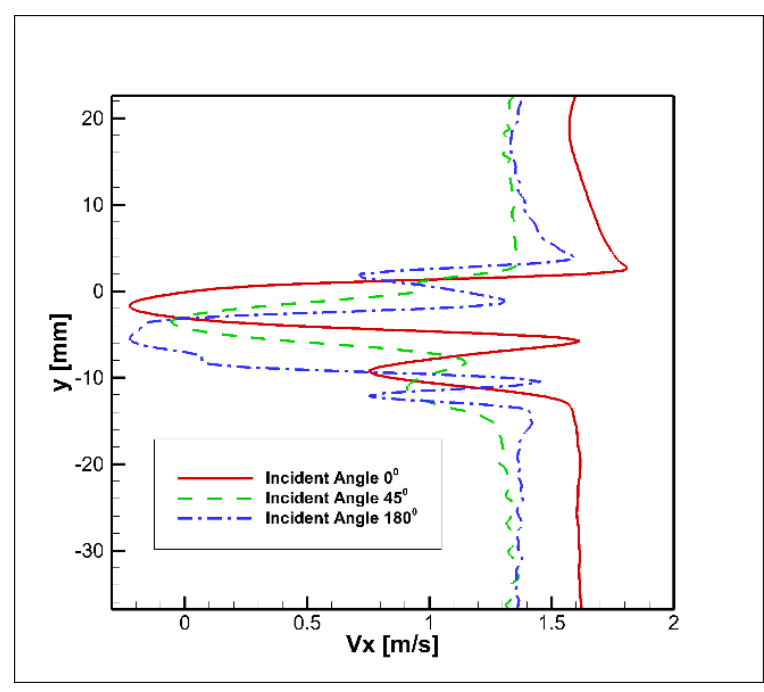

(a)

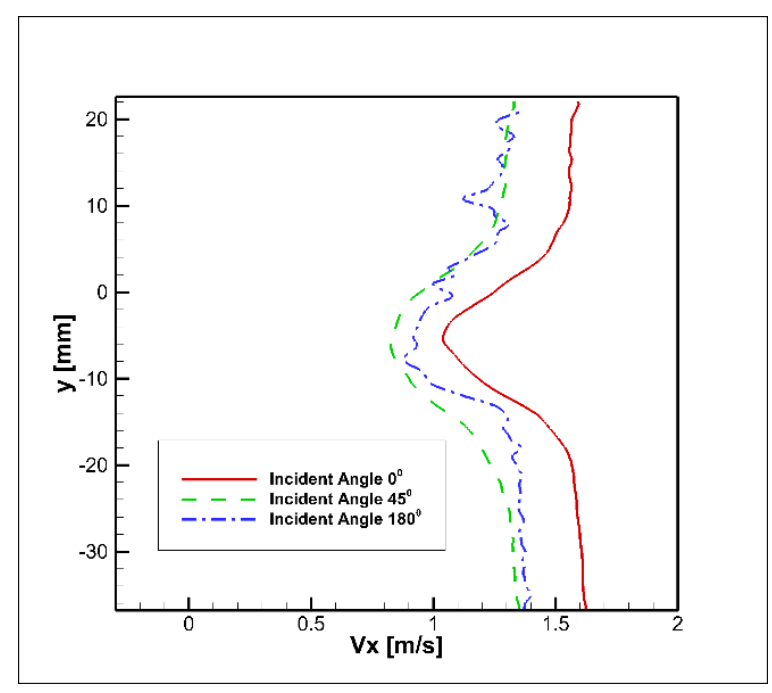

(c)

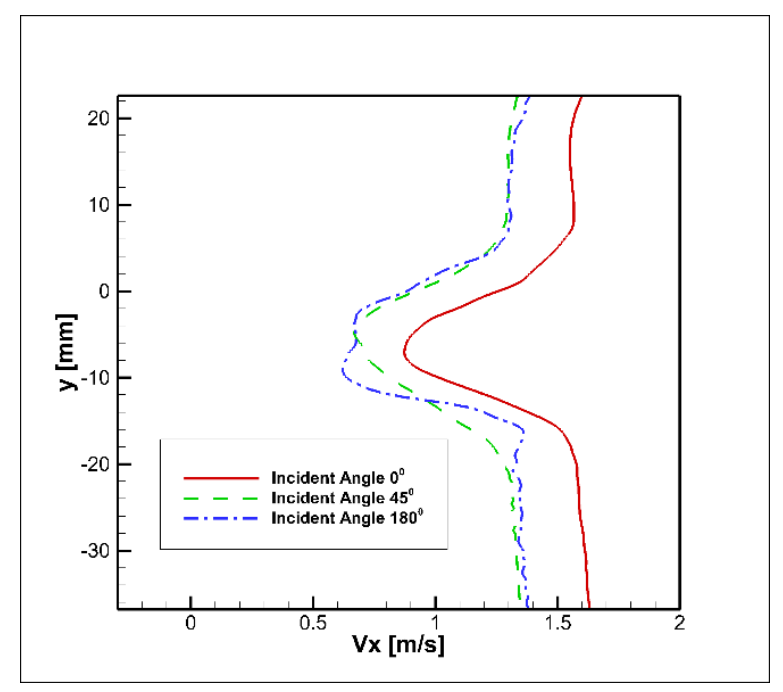

(b)

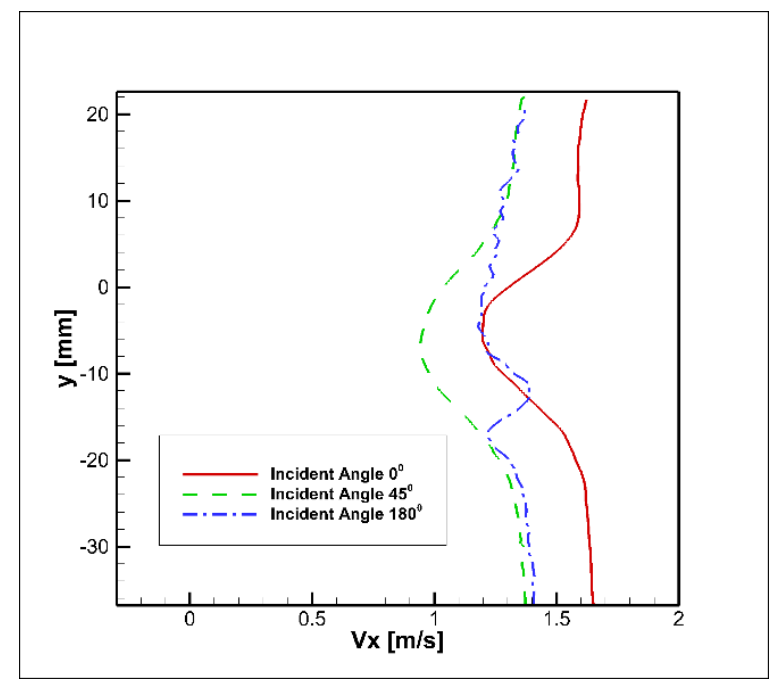

(d)

Figure 23. Average Streamwise velocity profile for $R e=57$ at position (a) $x / d=0$, (b) $x / d=12.9$, (c) $\mathrm{x} / \mathrm{d}=25.8$, and (d) 38.7 


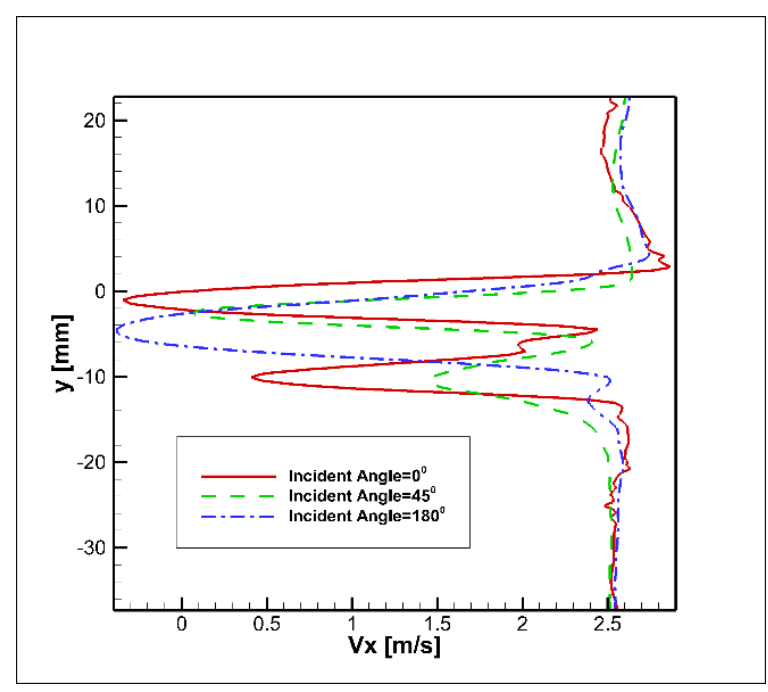

(a)

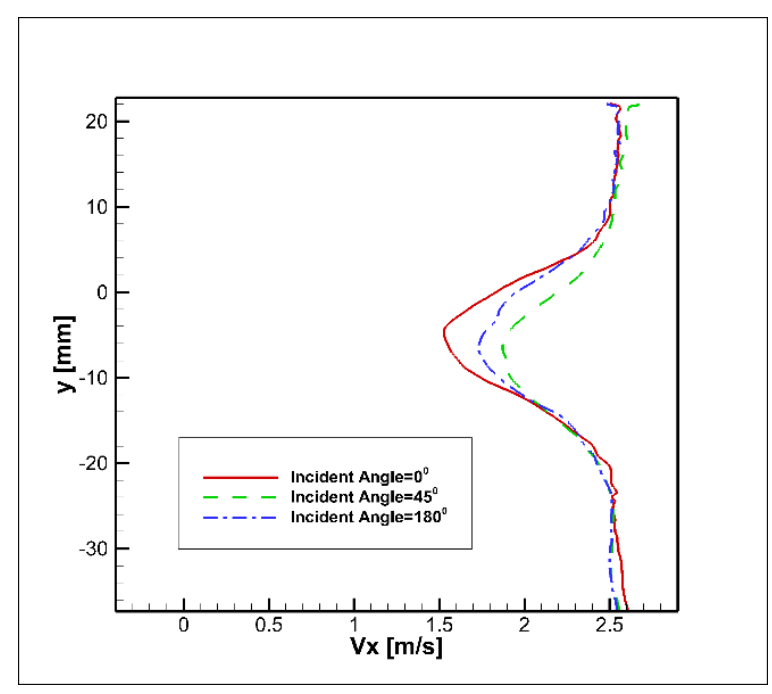

(c)

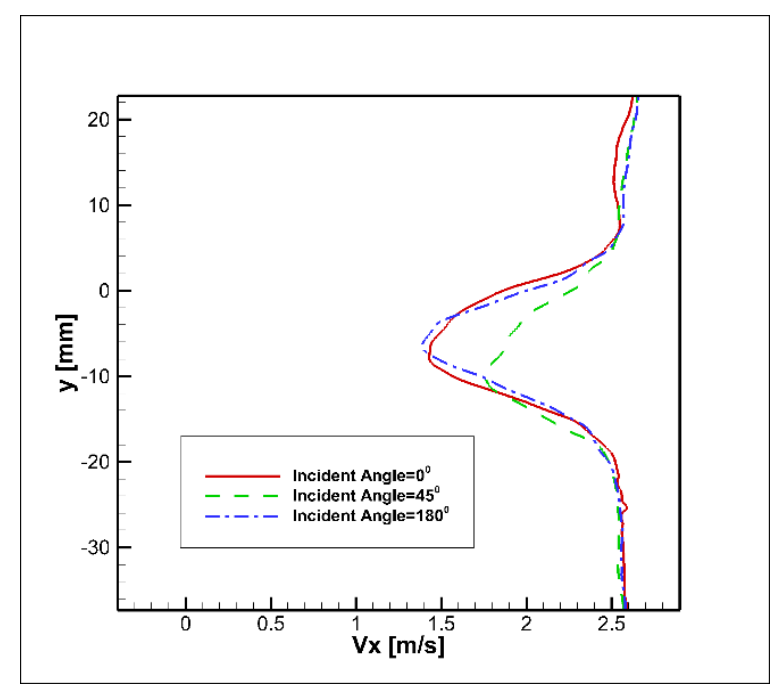

(b)

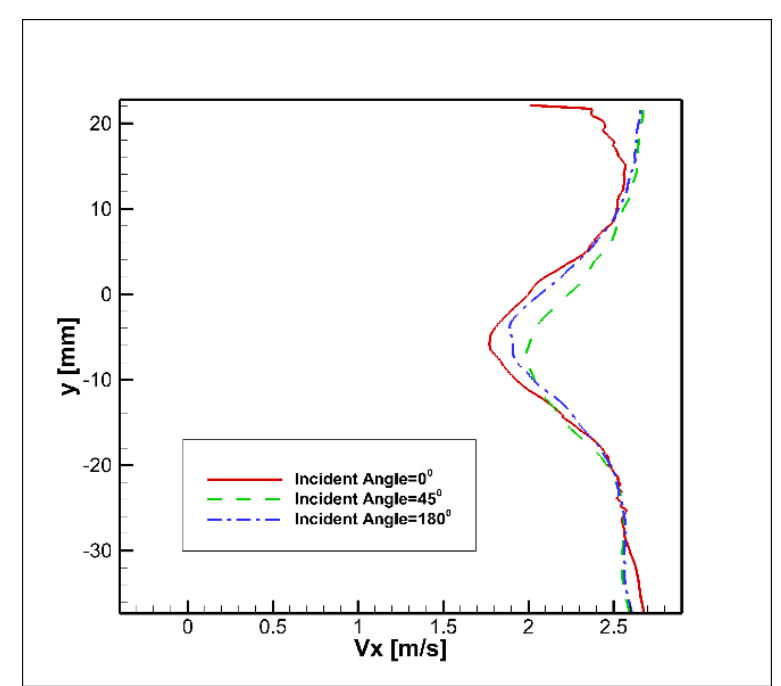

(d)

Figure 24. Average Streamwise velocity profile for $R e=500$ at position (a) $x / d=0$, (b) $x / d=12.9$, (c) $\mathrm{x} / \mathrm{d}=25.8$, and (d) 38.7 


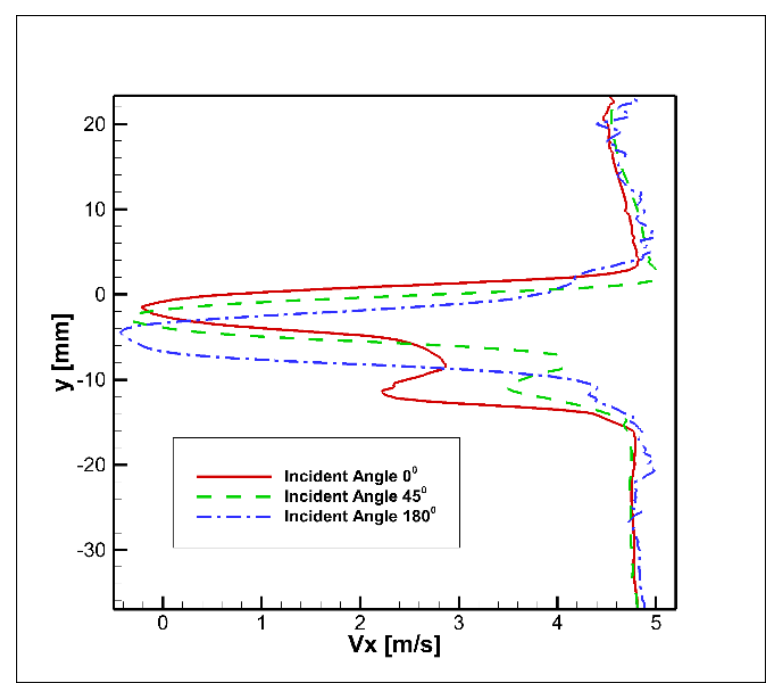

(a)

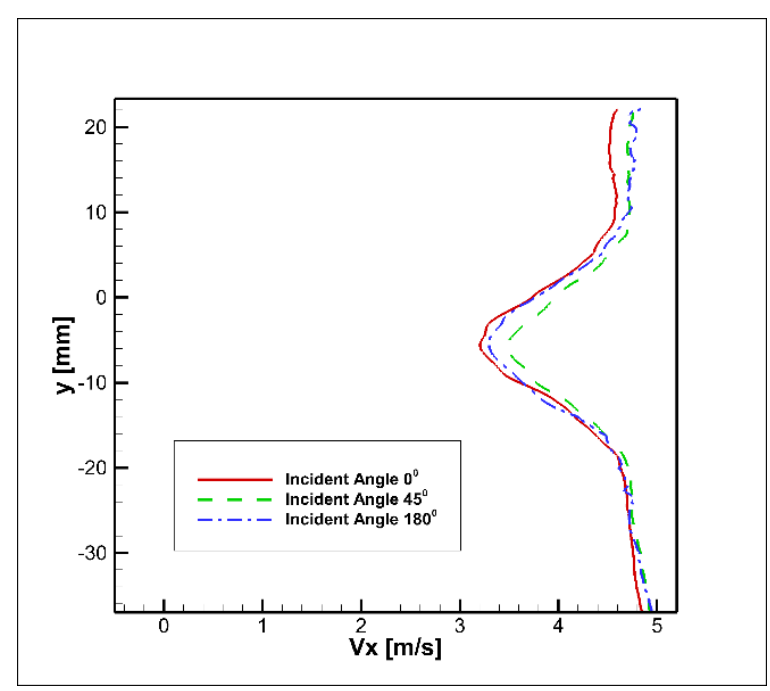

(c)

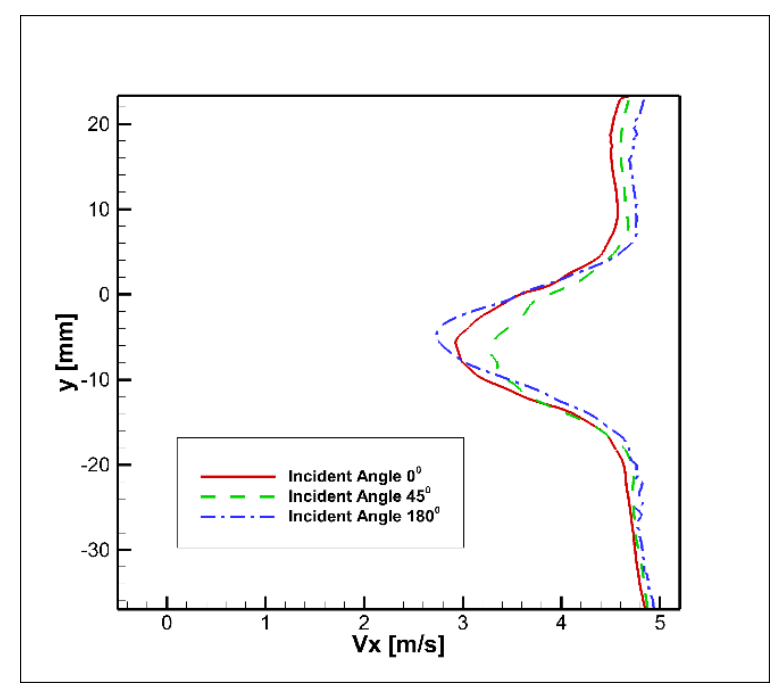

(b)

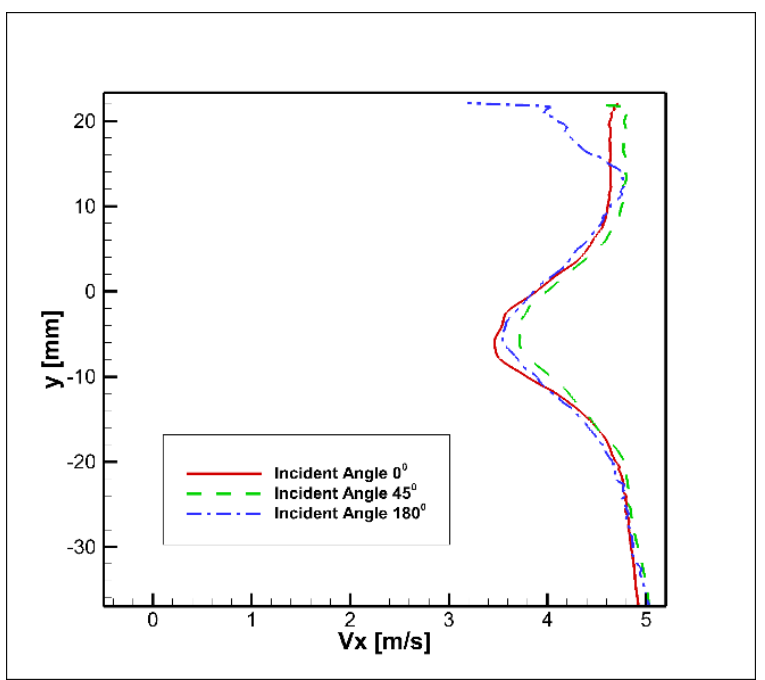

(d)

Figure 25. Average Streamwise velocity profile for $R e=858$ at position (a) $x / d=0$, (b) $x / d=12.9$, (c) $\mathrm{x} / \mathrm{d}=25.8$, and (d) 38.7 


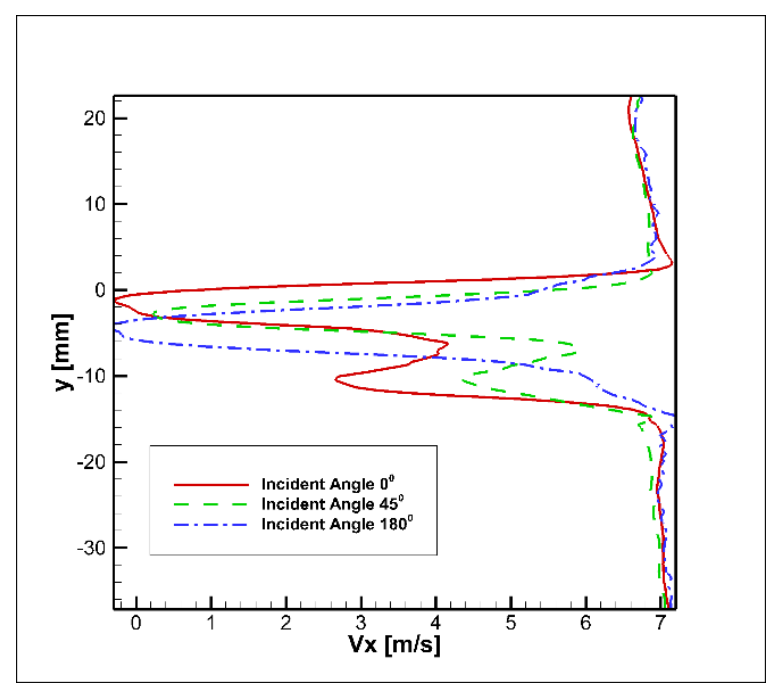

(a)

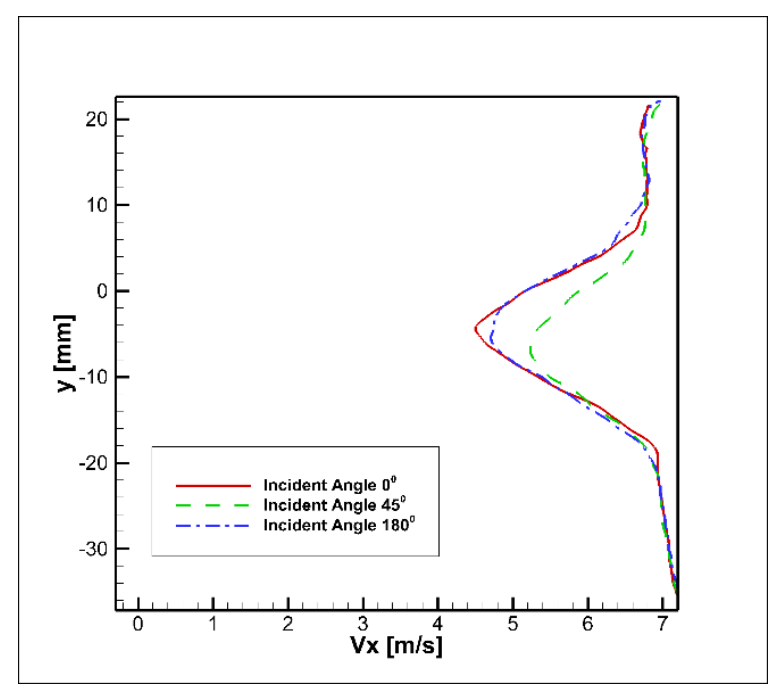

(c)

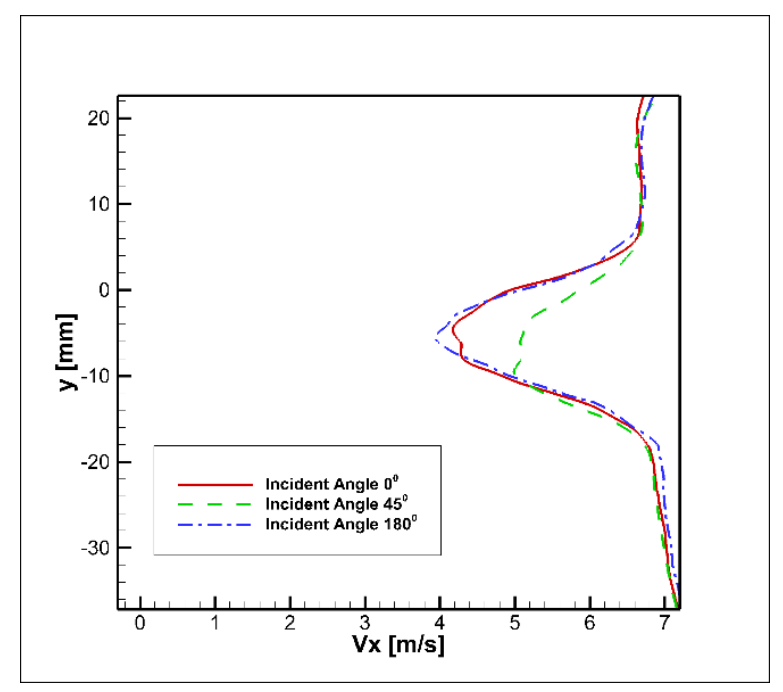

(b)

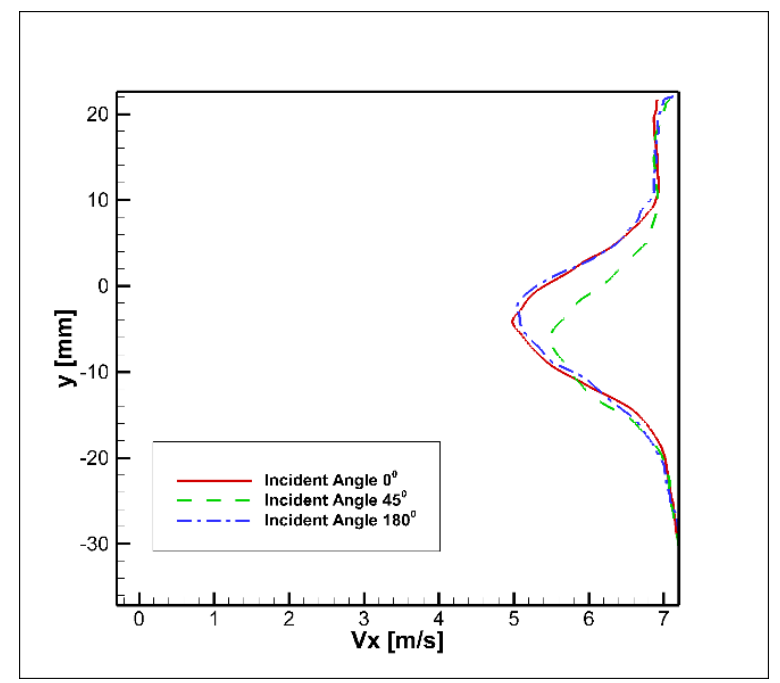

(d)

Figure 26. Average Streamwise velocity profile for $\mathrm{Re}=1250$ at position (a) $\mathrm{x} / \mathrm{d}=0$, (b) $\mathrm{x} / \mathrm{d}=12.9$, (c) $\mathrm{x} / \mathrm{d}=25.8$, and (d) 38.7 


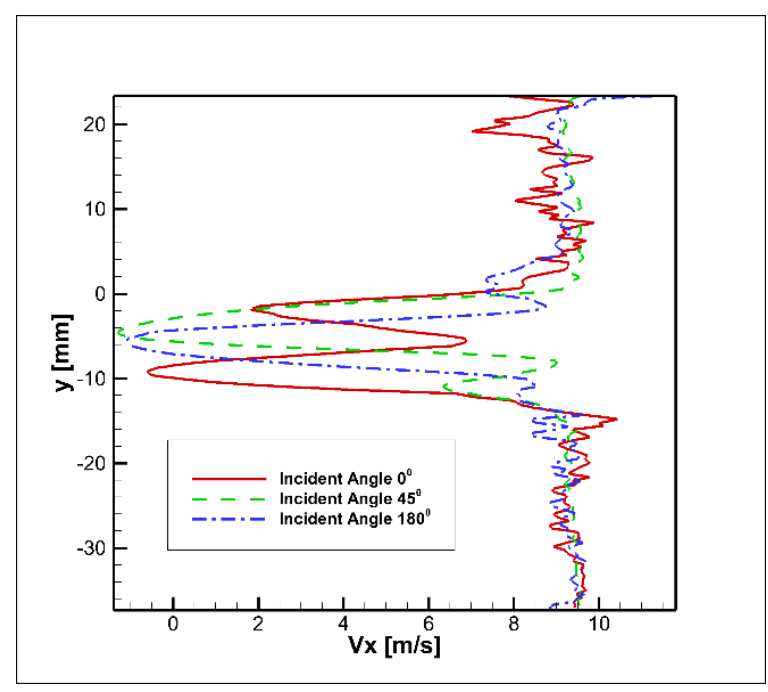

(a)

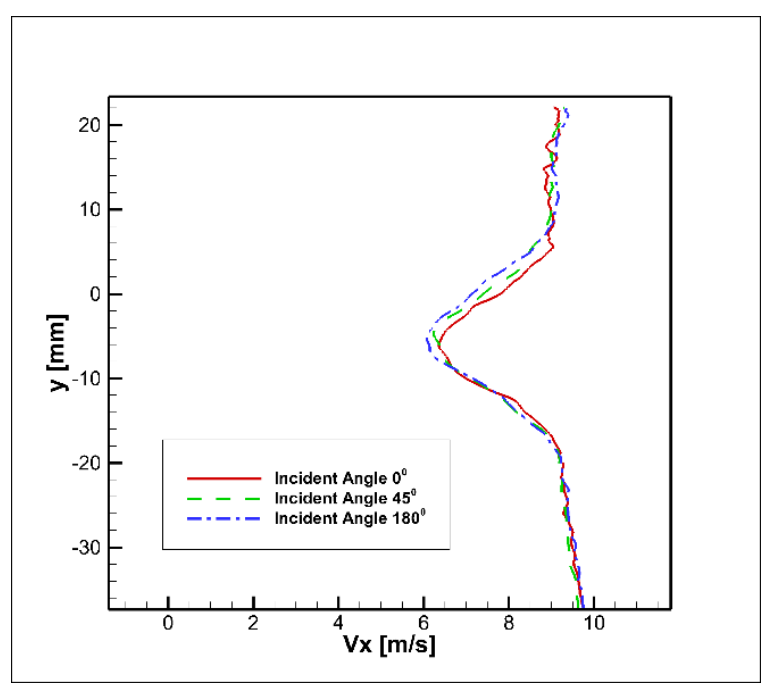

(c)

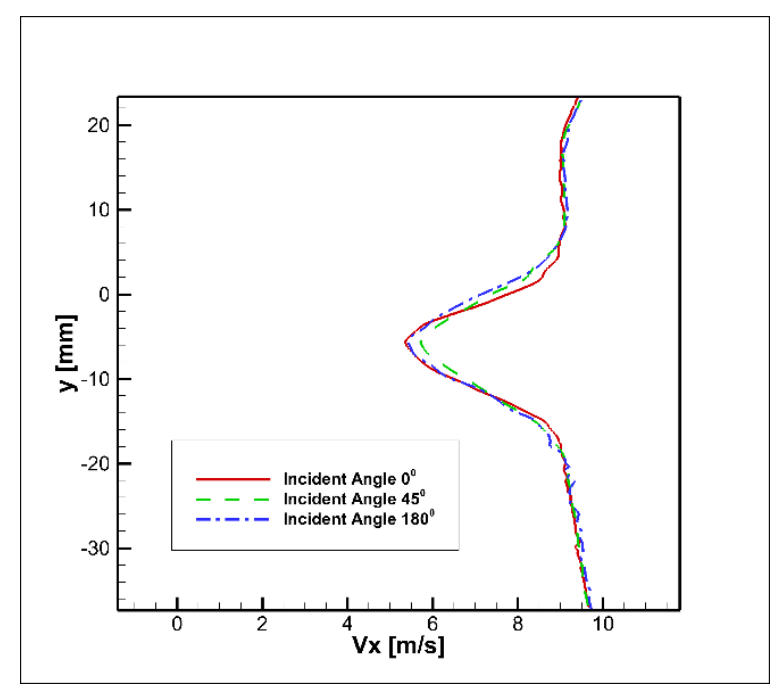

(b)

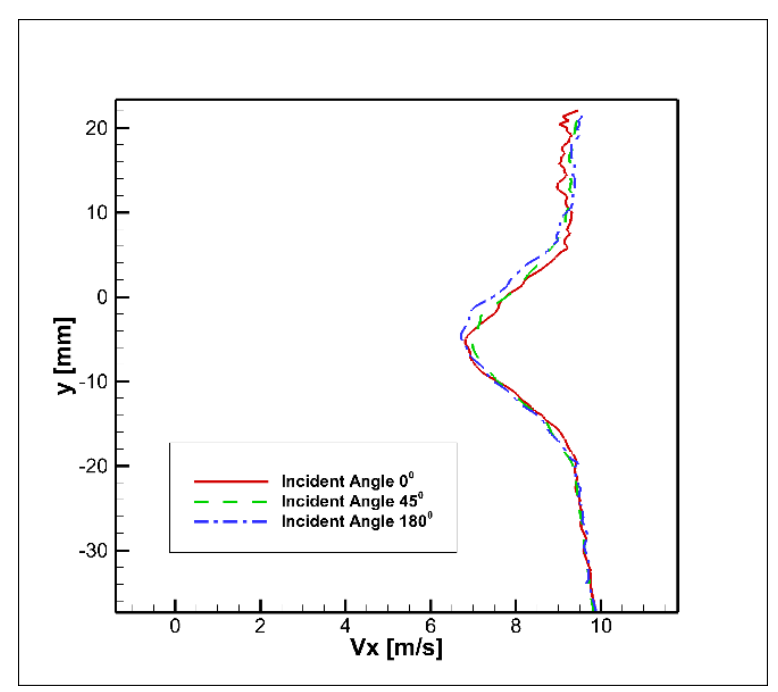

(d)

Figure 27. Average Streamwise velocity profile for $\mathrm{Re}=1700$ at position (a) $\mathrm{x} / \mathrm{d}=0$, (b) $\mathrm{x} / \mathrm{d}=12.9$, (c) $\mathrm{x} / \mathrm{d}=25.8$, and (d) 38.7 


\section{Rotating Cases: Velocity Contour and Wake Velocity Profile}

Figure 28 shows the instantaneous velocity plot of various $\mathrm{RPM}$ for $\mathrm{Re}=57$. And other sets of instantaneous velocity datas for $\mathrm{Re}=858,1250$ and 1700 are shown in APPENDIX-D. At low $\mathrm{Re}$, the rotation of cylinder system creates recirculation region in the flow domain. As the RPM increases these recirculation region appears to travel far downstream. When the Re number increases the wake region gets narrower compare to low Re number. All the instantaneous plot shows the velocity deficit in the wake region compare to the freestream. From the observation of 100 different instantaneous results for each of the condition shows that the wake is very unstready in nature. Due to counter clock wise rotation the wake is skewed upward. Some of the wake stucture are periodic during the rotation cycle of cylinder. In order to keep the discussion concise, one instantaneous plot from each condition is shown Figure 28shows region of high velocity spot that was marked in the blue box. This region coincide with the vorticity location which explains the reason of having high velocity magnitude in those space. In order to show how the RPM effects the flow structure, the average velocity contour is plotted in APPENDIX-E. The average velocity is calculated based on 100 instantaneous results. Note that the instantaneous results are time uncorelated. The average velcoity contour for all the caeses show the velocity deficit in the wake region. At $\mathrm{Re}=57$, a recirculation region form in the average velocity profile. The recirculation region appears to be skewed upward due to the counter clock wise rotation of cylinder system. As the RPM increases for $\mathrm{Re}=57$, the recirculation region becomes more stronger.As the Re number increases, the recirculation region dissappears. In addition, the wake in the average velocity behind a rotating cylinder resemble with single cylinder wake. Apart from these features, with varying RPM, the average velocity contour does not show any significant difference. However, as the Re number increases the velocity deficit also increaes and the wake region gets 
narrower. Although the velocity contour doesnot show significant difference, the velocity profile at different downstream position shows distinct pattern. We have non dimensionalized the x coordinate of velocity profiles with respect to diameter of the circle excribed to the triangle having same dimenstion as the cylidner arrangement. The non-dimensional locations where the velocity profiles are plotted are $x / D=0, x / D=4.13, x / D=8.26$, and $x / D=12.4$. For a specific Re number, the velocity plots were drawn in the same scale for different downstream location to understand how the velocity magnitude varies as we move from upstream to downstream. Figure 29 and Figure 30 shows the velocity profile of different RPM at four different downstream location when the $\mathrm{Re}=57$ and 500. At low Re number, the velocity profile in upstream shows that with increasing RPM the velocity deficit increases. The profile of velocity also indicates the unsteady nature of the flow due to the vortex interaction. As the we move to further downstream the velocity deficit decreases and the profiles starts to look similar for various RPM. At high Re number such as 858, 1250, and 1700, in Figure 31 to Figure 33, the velocity profile starts to become similar for varying RPM. The pronounced double peaks seen previously in non-rotating cases are not so pronounced in rotating cases. Due to the rotating of cylinder system, the wake mixing creates flow structure which are not phase locked. That's why the average velocity profile does not show much pronounced velocity peaks. 


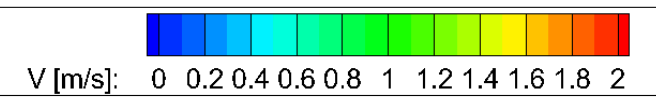

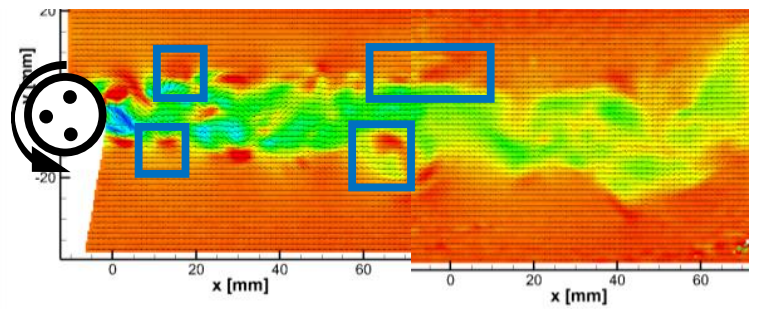

(a)

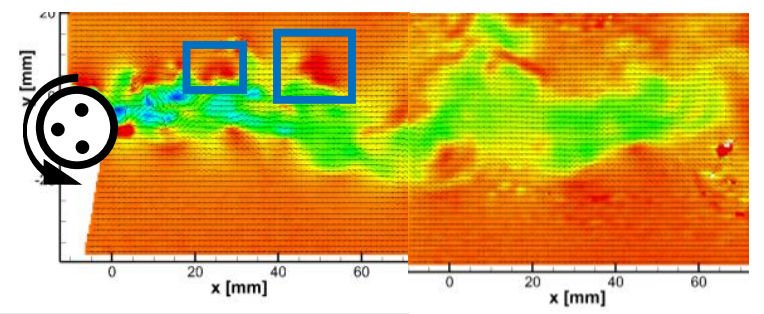

(c)

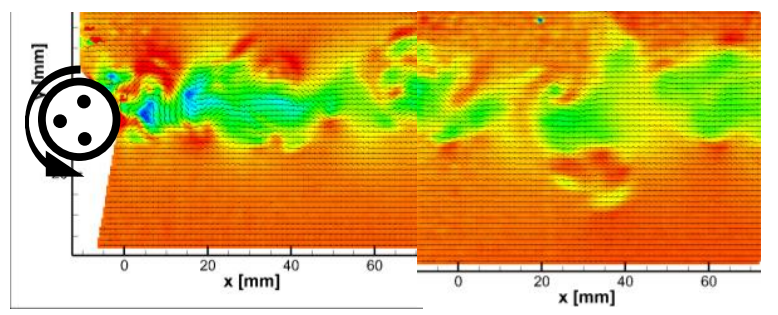

(e)

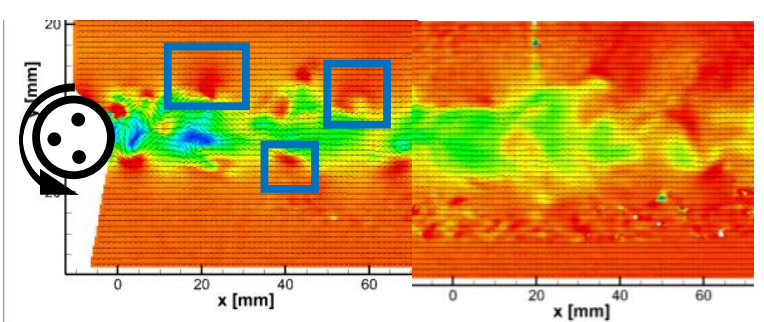

(b)

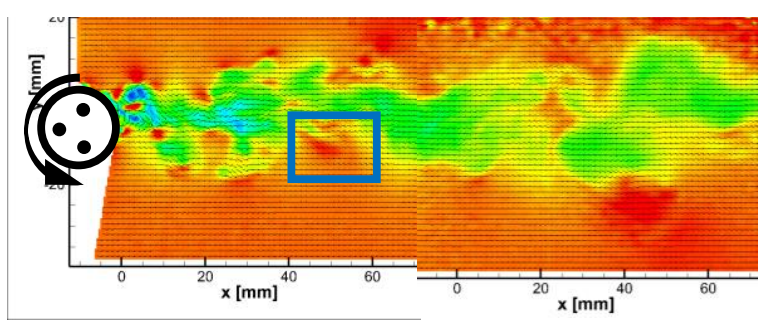

(d)

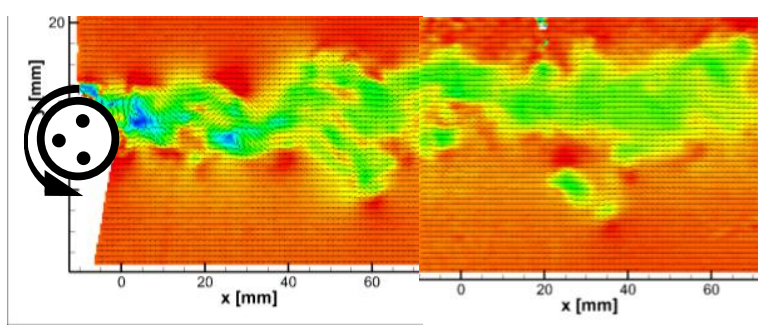

(f)

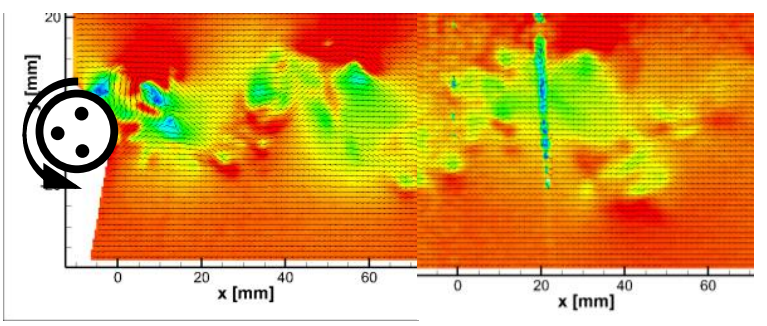

(g)

Figure 28. Instantaneous Velocity Contour at $\mathrm{Re}=57$ and Rotation Speed is (a) 120 (b) 360 (c) 960 (d) 1500 (e) 1800 (f) 2400 (g) 3000 RPM 


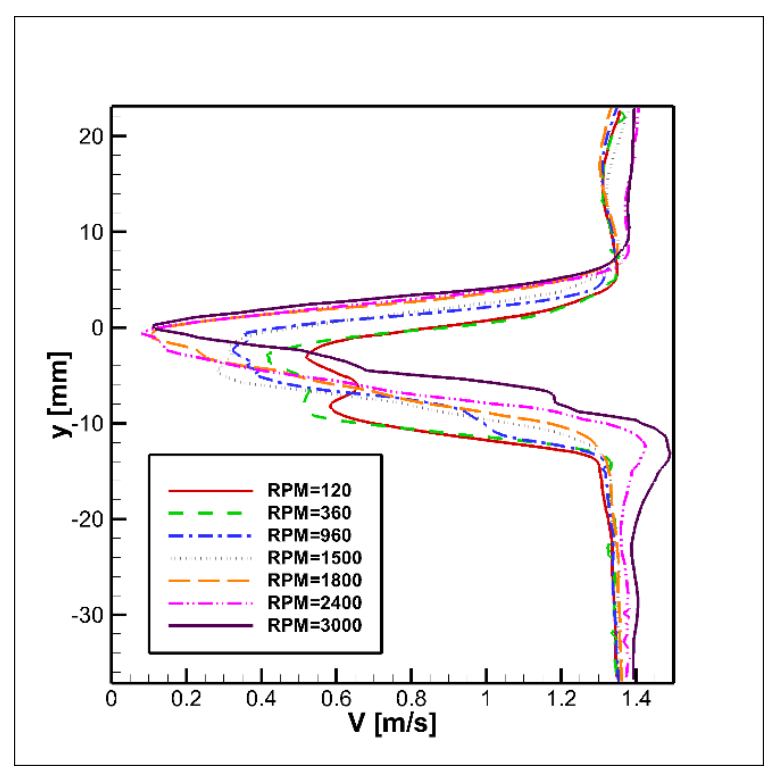

(a)

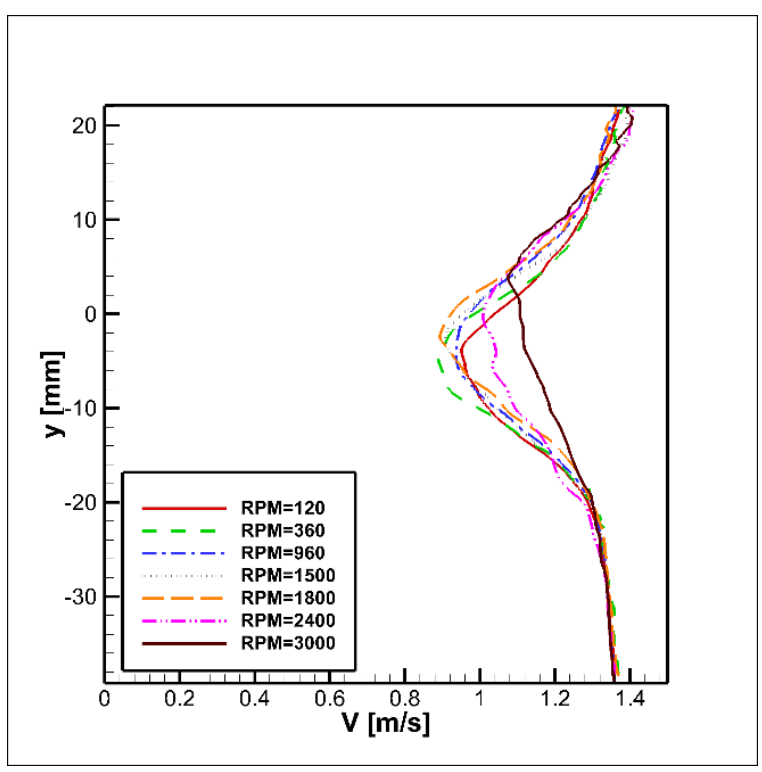

(c)

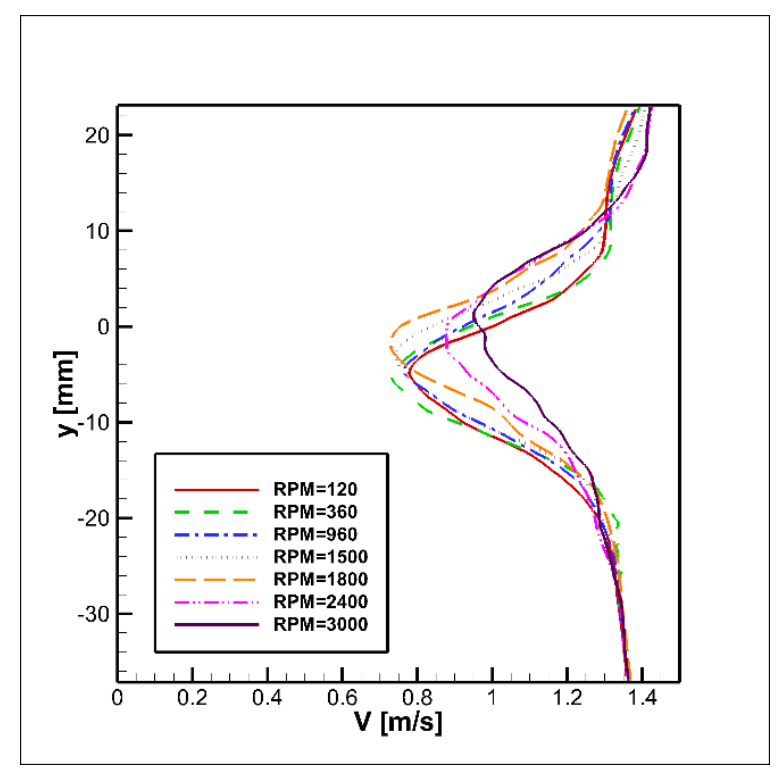

(b)

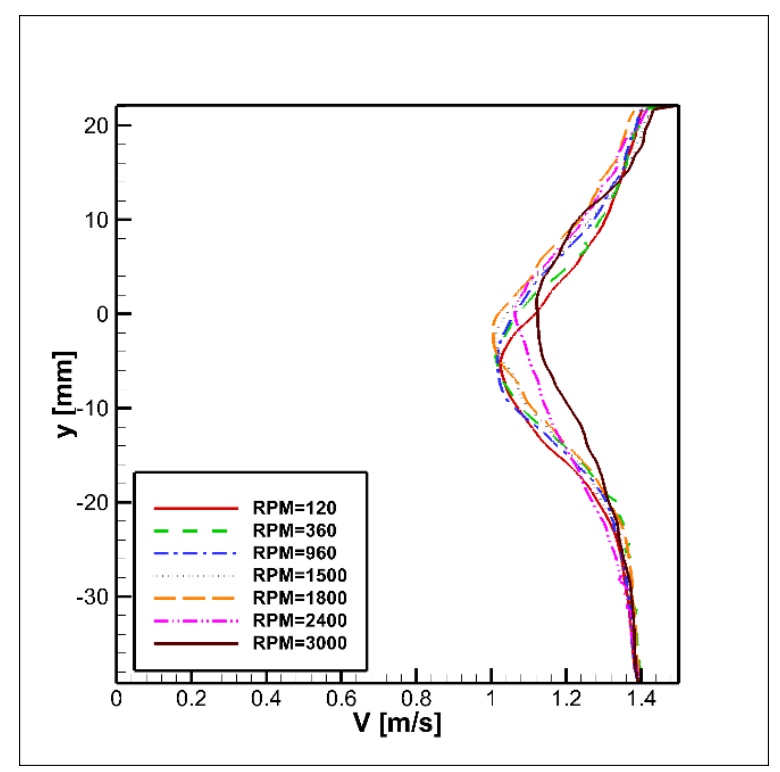

(d)

Figure 29. Velocity profile for $\mathrm{Re}=57$ at location (a) $\mathrm{x} / \mathrm{D}=0$ (b) $\mathrm{x} / \mathrm{D}=4.13$ (c) $\mathrm{x} / \mathrm{D}=8.26$ (d) $\mathrm{x} / \mathrm{D}=12.4$ 


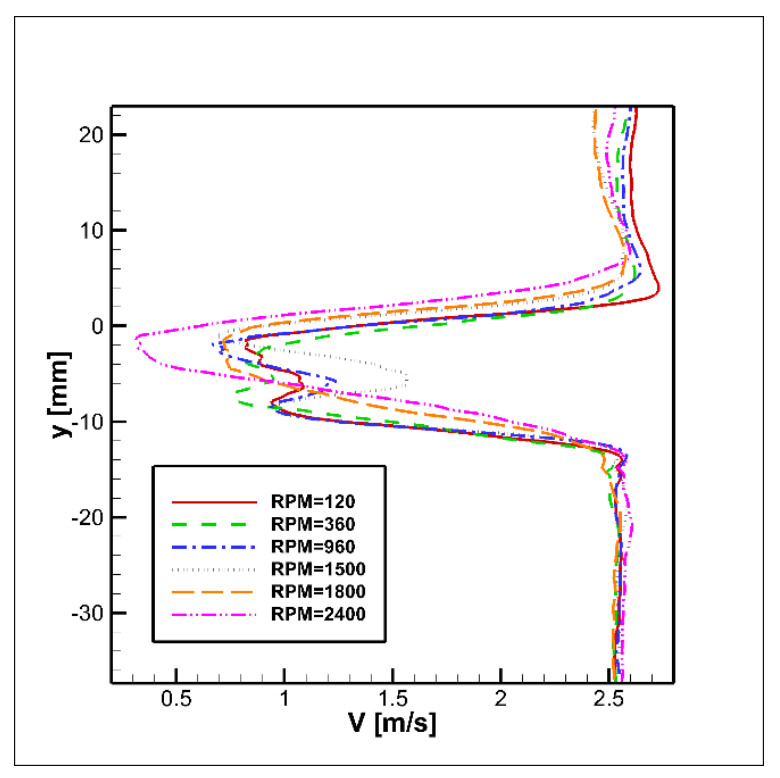

(a)

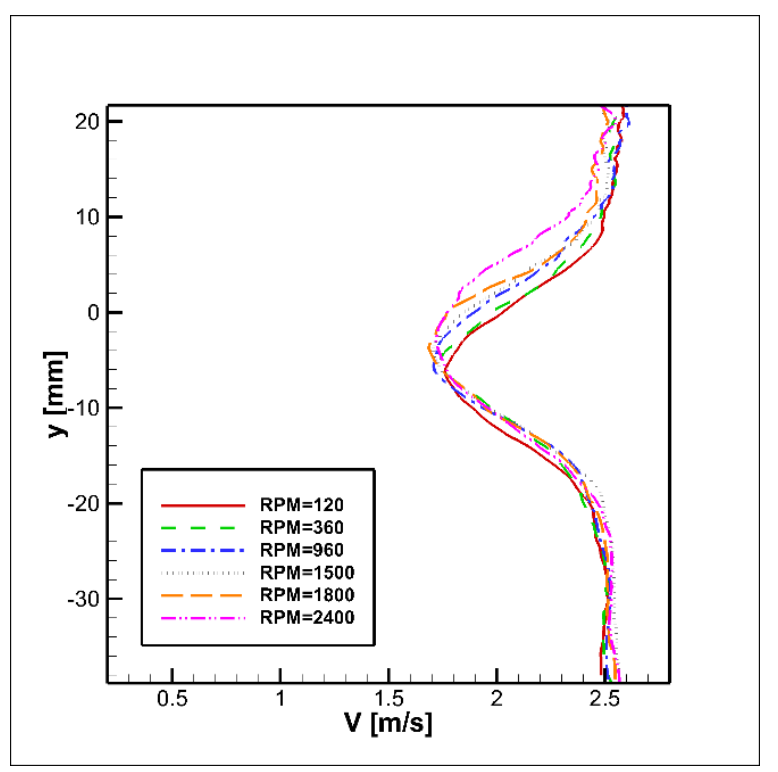

(c)

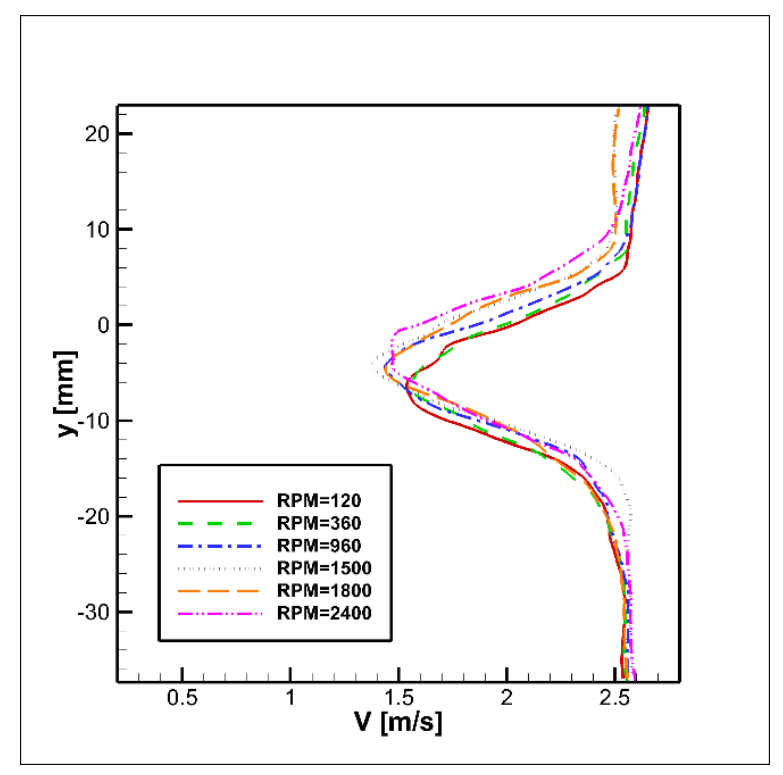

(b)

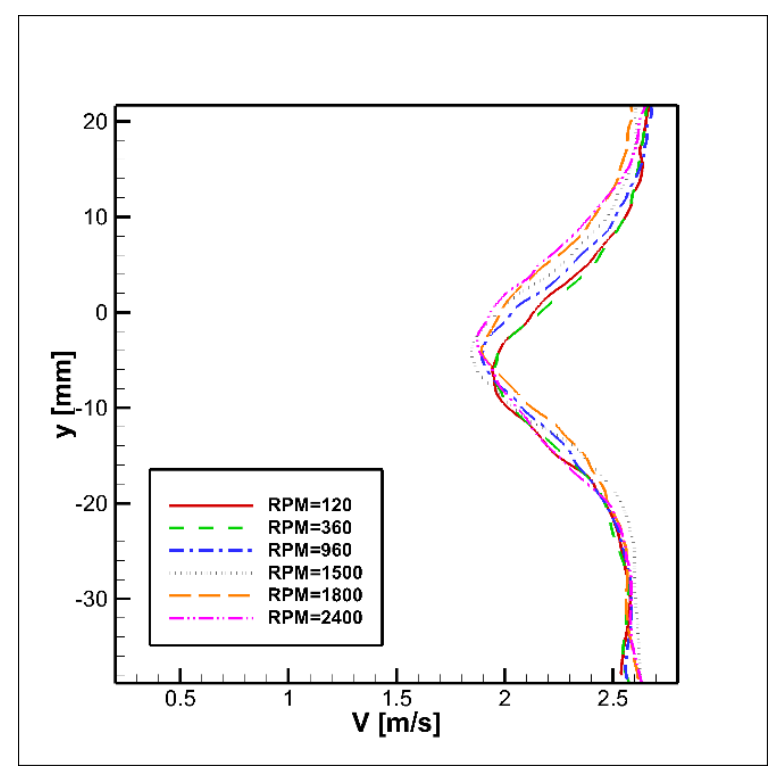

(d)

Figure 30. Velocity profile for $\mathrm{Re}=500$ at location (a) $\mathrm{x} / \mathrm{D}=0$ (b) $\mathrm{x} / \mathrm{D}=4.13$ (c) $\mathrm{x} / \mathrm{D}=8.26$ (d) $\mathrm{x} / \mathrm{D}=12.4$ 


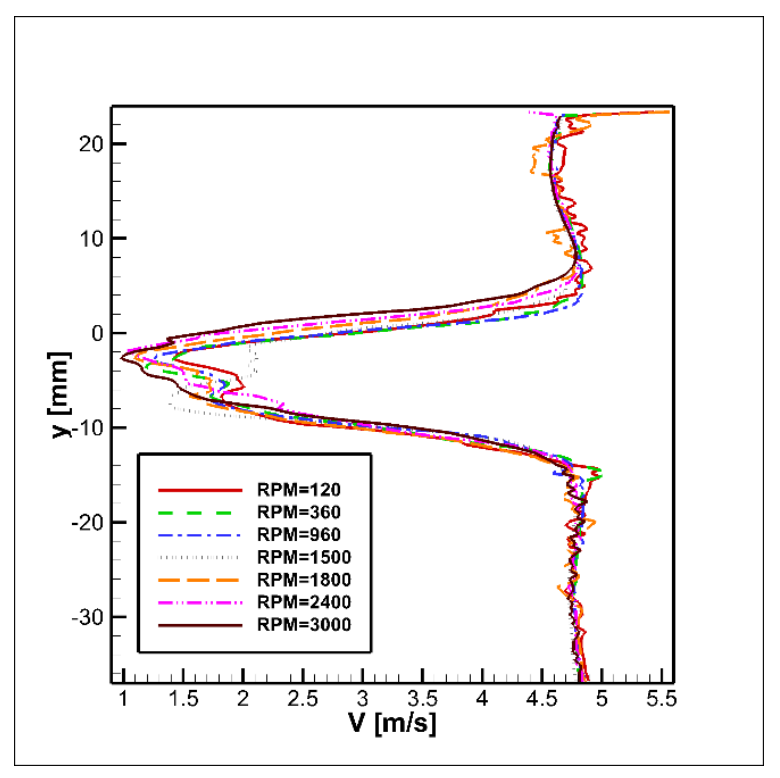

(a)

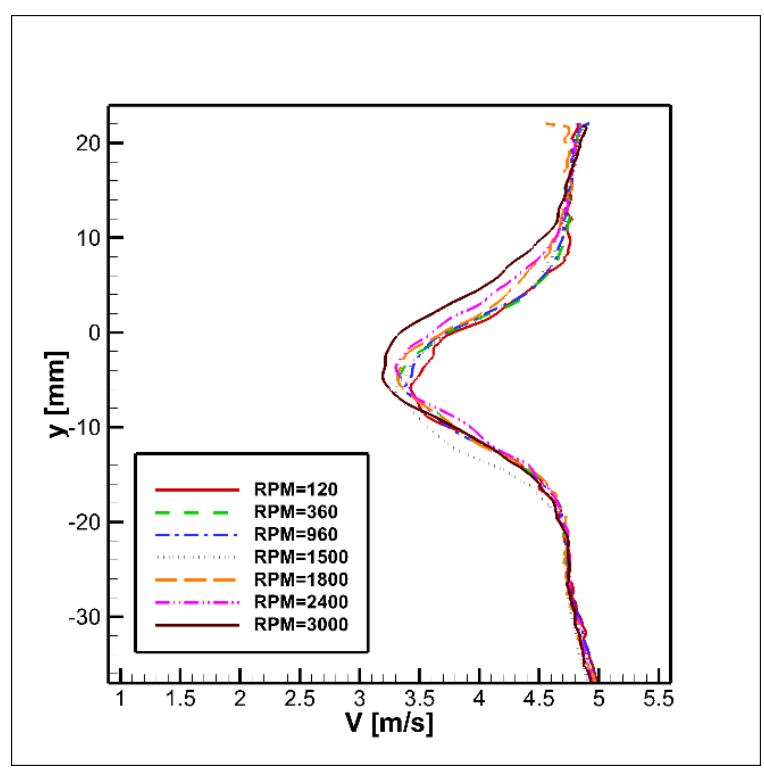

(c)

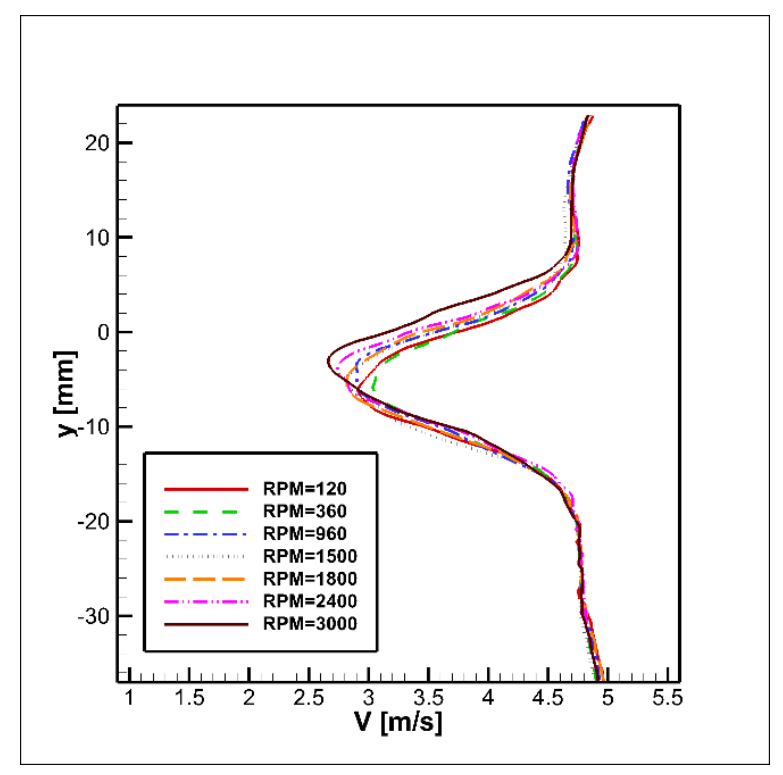

(b)

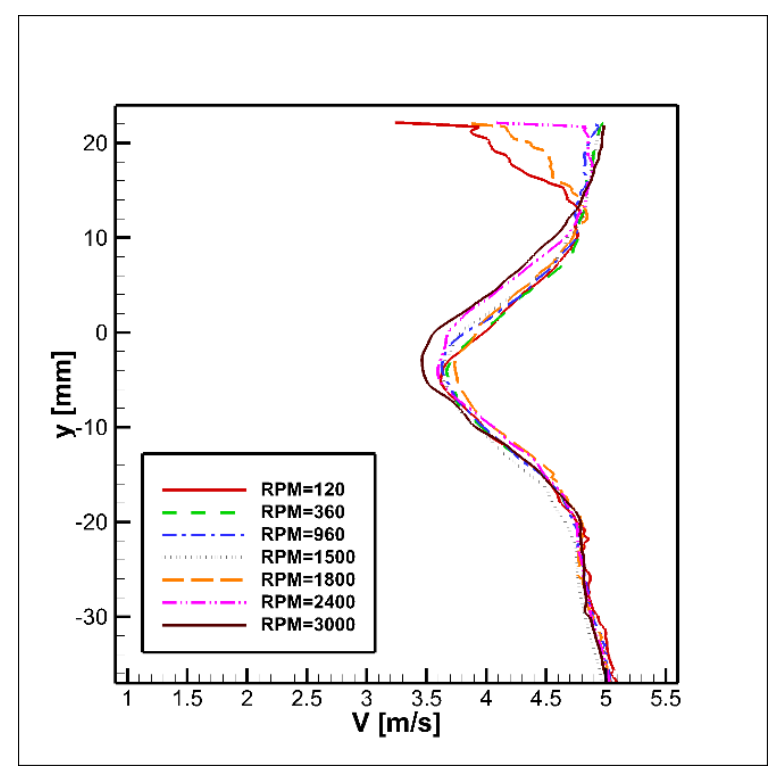

(d)

Figure 31. Velocity profile for $\mathrm{Re}=858$ at location (a) $\mathrm{x} / \mathrm{D}=0$ (b) $\mathrm{x} / \mathrm{D}=4.13$ (c) $\mathrm{x} / \mathrm{D}=8.26$ (d) $\mathrm{x} / \mathrm{D}=12.4$ 


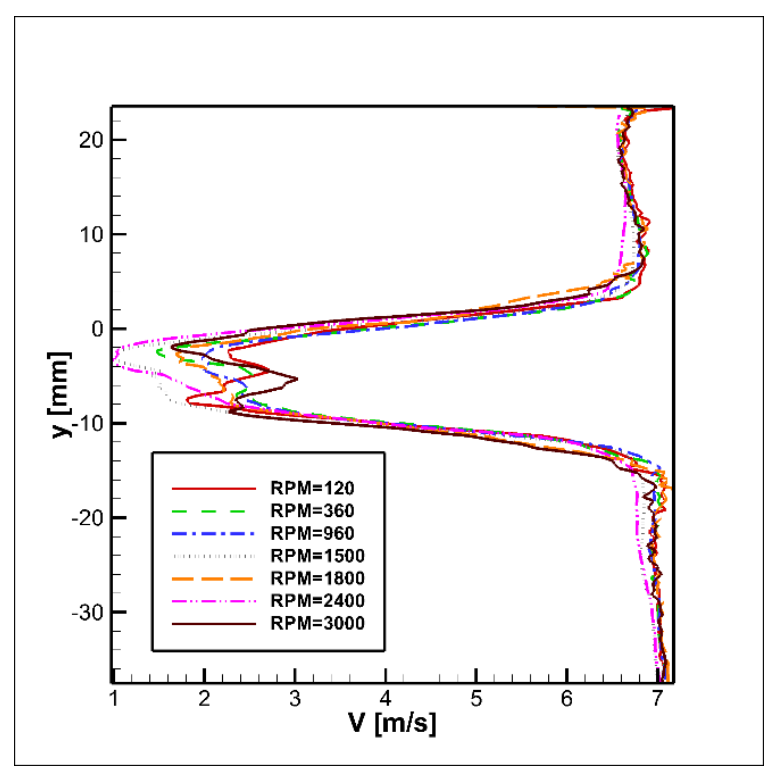

(a)

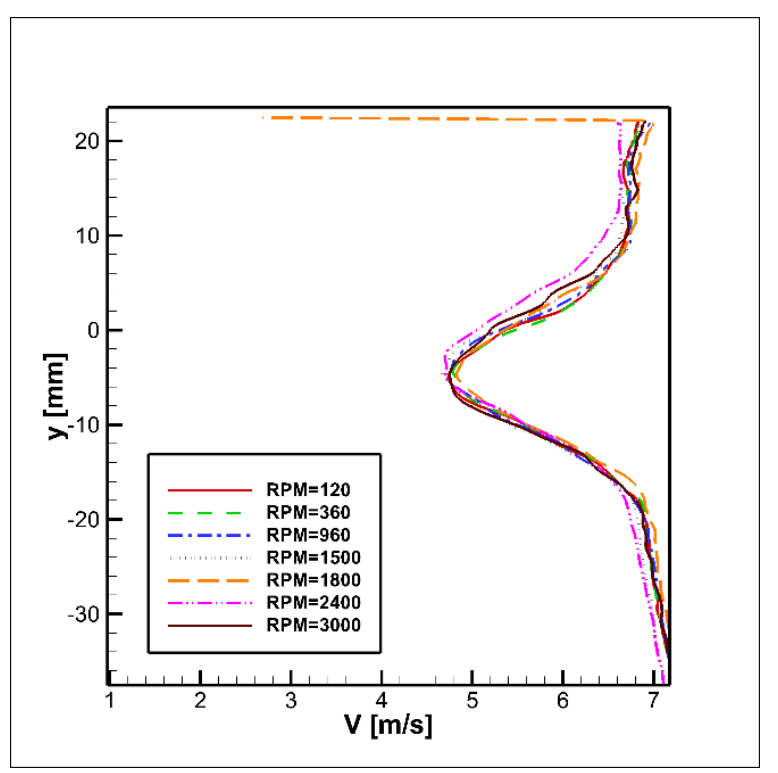

(c)

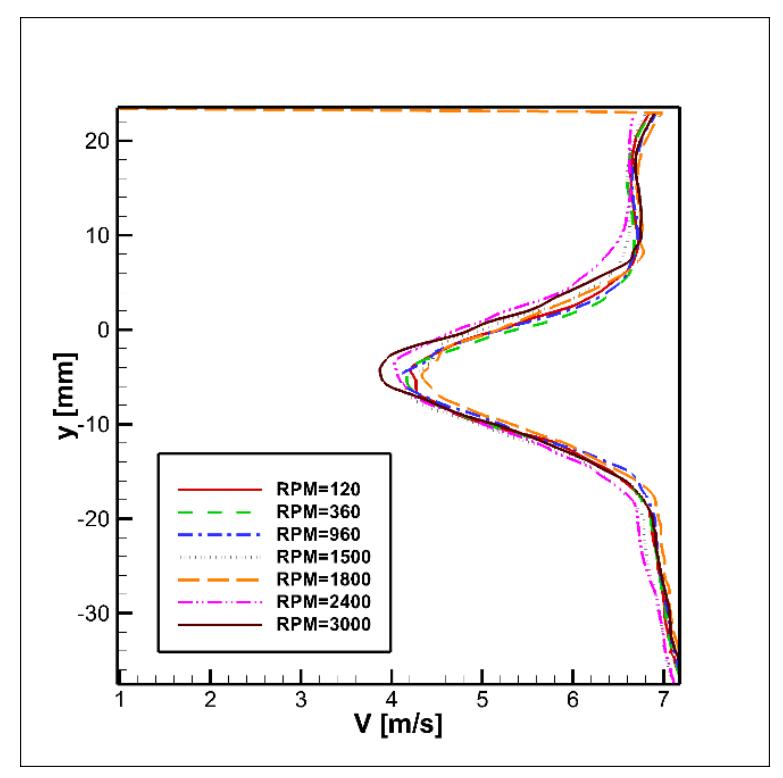

(b)

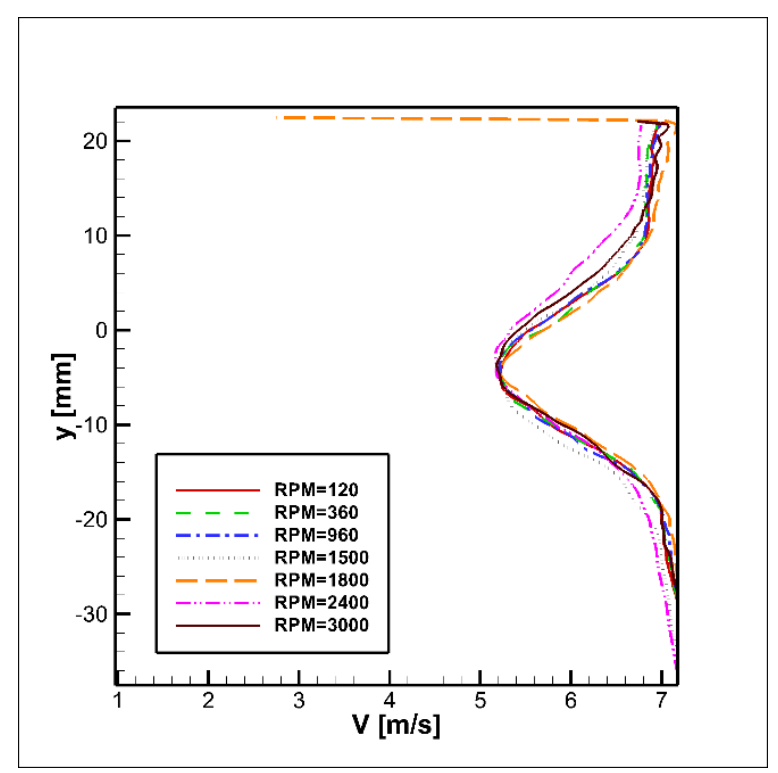

(d)

Figure 32. Velocity profile for $\mathrm{Re}=1258$ at location (a) $\mathrm{x} / \mathrm{D}=0$ (b) $\mathrm{x} / \mathrm{D}=4.13$ (c) $\mathrm{x} / \mathrm{D}=8.26$ (d) $\mathrm{x} / \mathrm{D}=12.4$ 


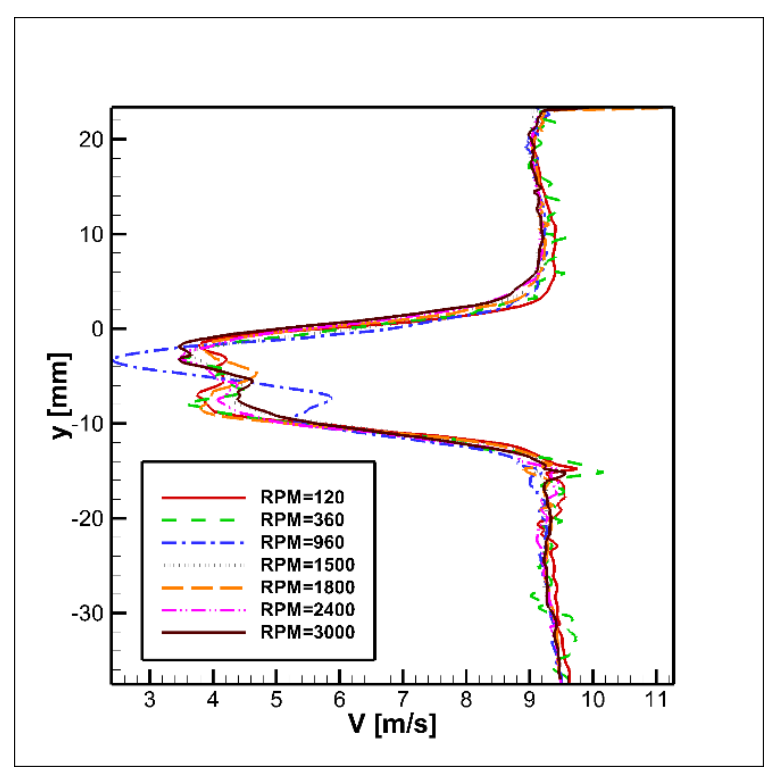

(a)

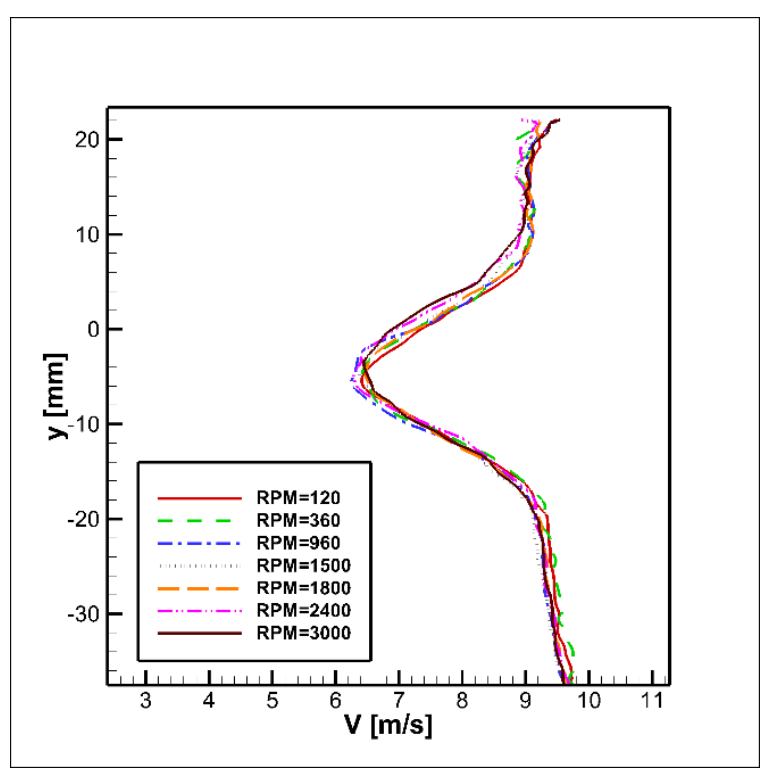

(c)

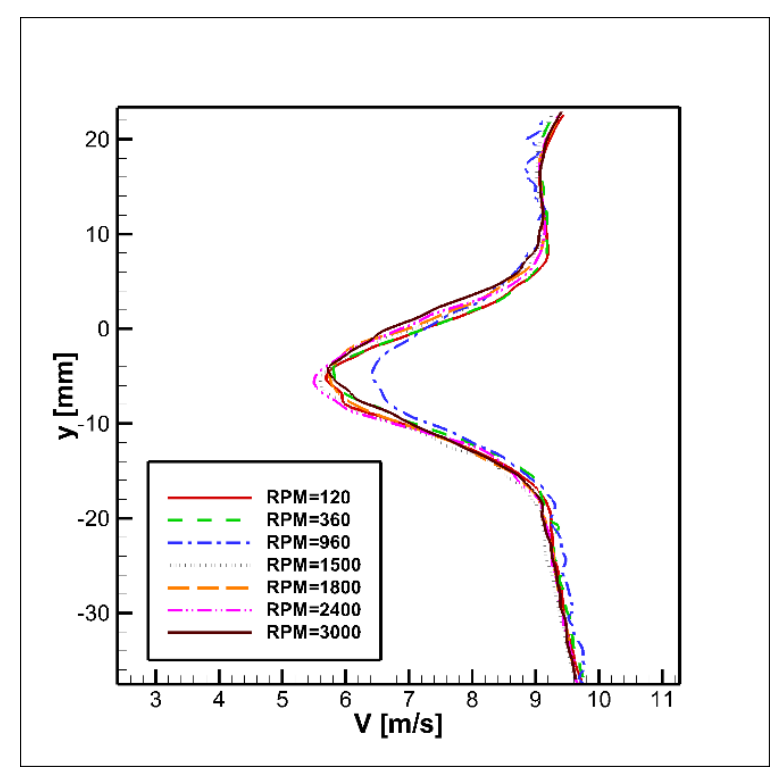

(b)

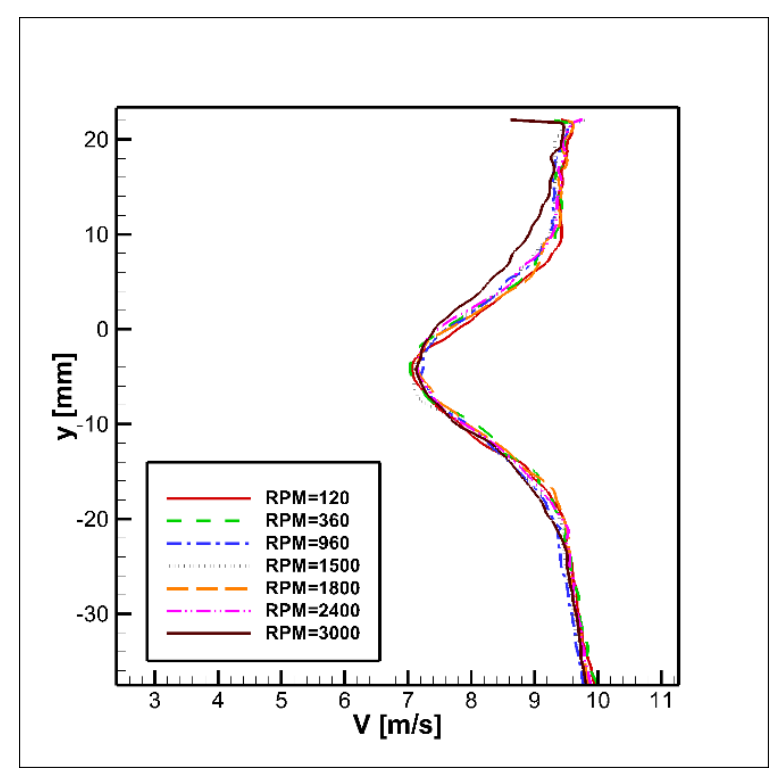

(d)

Figure 33. Velocity profile for $\mathrm{Re}=1700$ at location $(\mathrm{a}) \mathrm{x} / \mathrm{D}=0$ (b) $\mathrm{x} / \mathrm{D}=4.13$ (c) $\mathrm{x} / \mathrm{D}=8.26$ (d) $\mathrm{x} / \mathrm{D}=12.4$ 


\section{Rotating Cases: Vorticity Contour and Standard Deviation}

Figure 34 and Figure 35 shows the vorticity contour for $\operatorname{Re}=57$ and 858 . At $\operatorname{Re}=57$, due to the rotation, the cylinder wakes breaks into smaller positive and negative vortices. After the interaction of positive and negative vortices in the downstream, the instantaneous plot shows distinct vortex shedding as the RPM varies. With the change of RPM, each instances start to exhibit distinct pattern of vortex shedding. From all the instantaneous plot it proves that high wake mixing due to cylinder rotation makes the flow highly unsteady in nature. At $\operatorname{Re}=858$ in Figure 35 , the flow become highly turbulent and it does not show any distinct feature as the RPM varies. Figure 34 shows typical wake pattern. Figure 34 (b) with 360 RPM shows the wake pattern is at almost constant distance apart. The cluster of structures are much more chaotic and this increases as the RPM increase (in Figure 34 (b)). The average distance of wake cluster in Figure 34 (b) is found to be $18 \mathrm{~mm}$. which corresponds to frequency of $f=U / \lambda=1.5 / .018 \approx 83 \mathrm{~Hz}$ which corresponds to the $S t=f D / U=83 * .003 / 1.5=0.16$.

Figure 36 and Figure 37 shows the standard deviation plot for various RPM at $\operatorname{Re}=57$, 1700. And other standard deviation plot for intermediate Re number $=, 858,1250$ is shows in APPENDIX-F. At Re=57, the StDV contour changes slightly at downstream with varying RPM. When the RPM is at Maximum the fluctuation of velocity occur toward the upper side in the flow domain more compare to the other RPM. However, as the Re number increases the StDV contour in Fig 51 and 53 (a-g) does not show any significant difference. From the comparison of standard deviation with Re number, the result indicate that with increase Re number the turbulence intensity increases close to the cylinder system. 


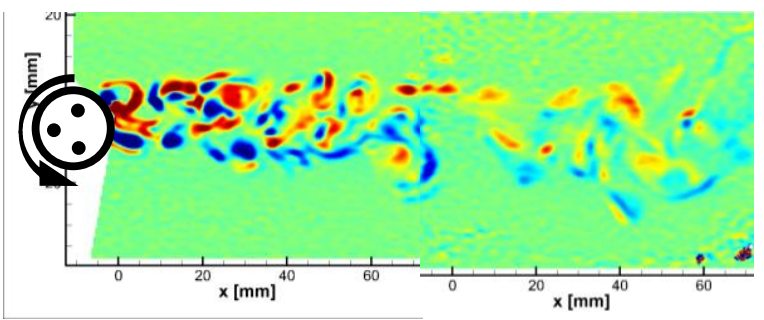

(a)

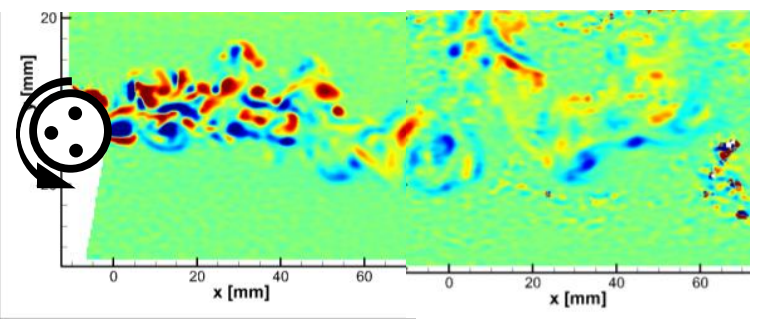

(c)

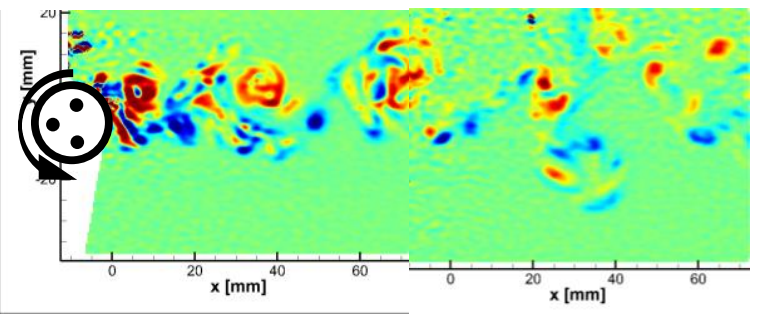

(e)

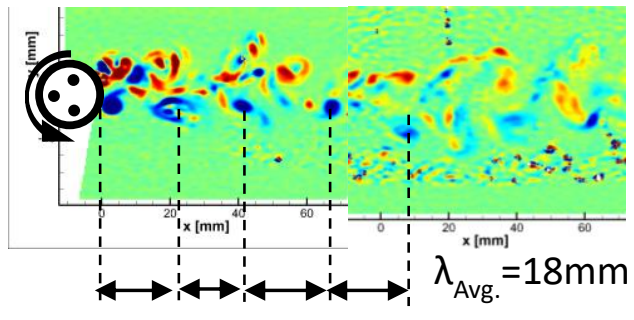

(b)

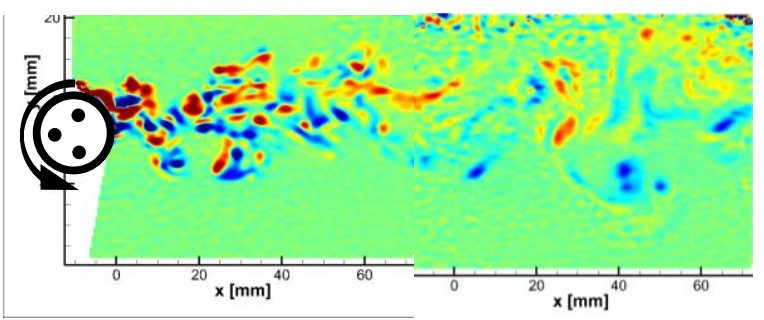

(d)

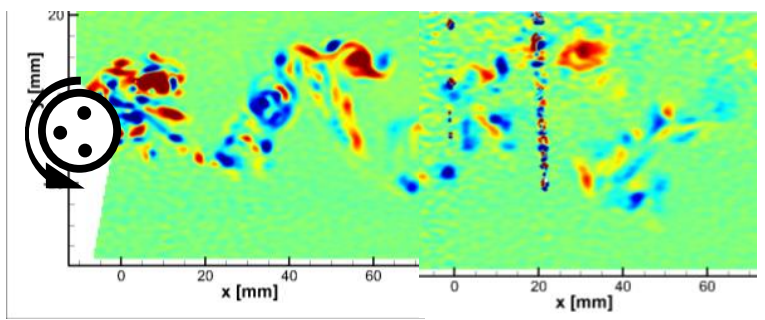

(f)

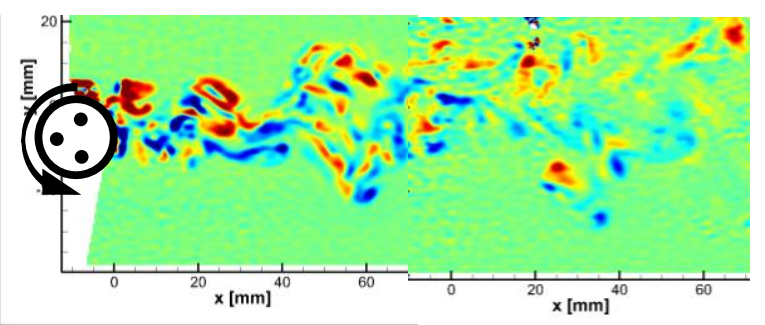

$(\mathrm{g})$

Figure 34. Instantaneous Vorticity Contour at $\mathrm{Re}=57$ and Rotation Speed is (a) 120 (b) 360 (c) 960 (d) 1500 (e) 1800 (f) 2400 (g) 3000 RPM 


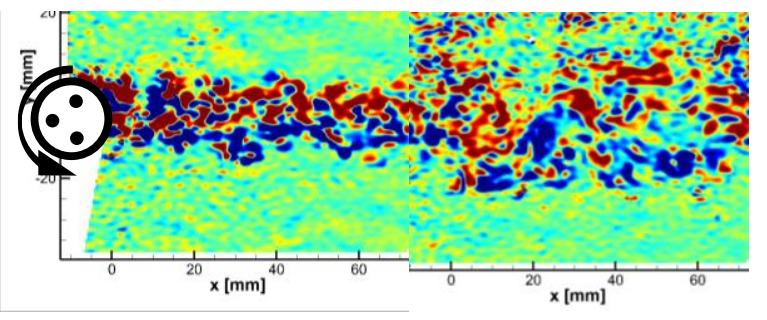

(a)

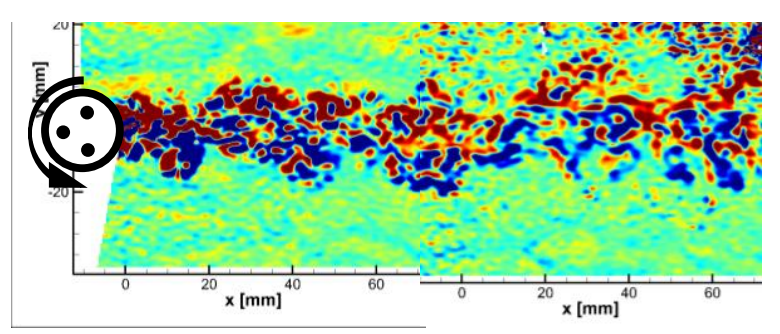

(b)

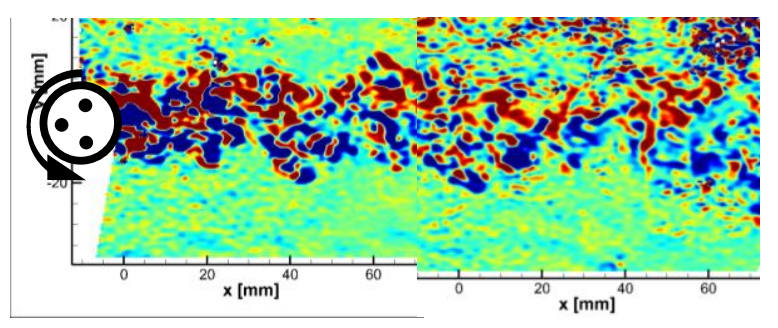

(e)

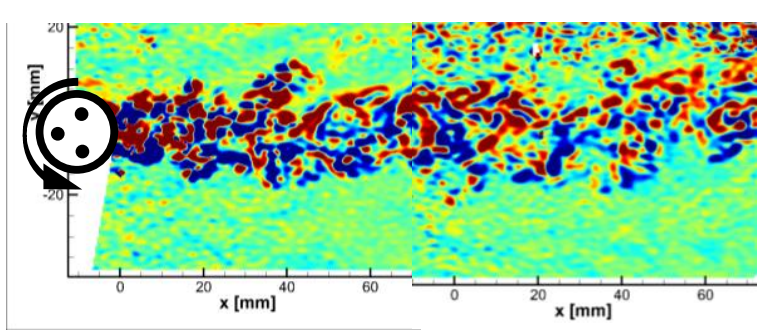

(b)

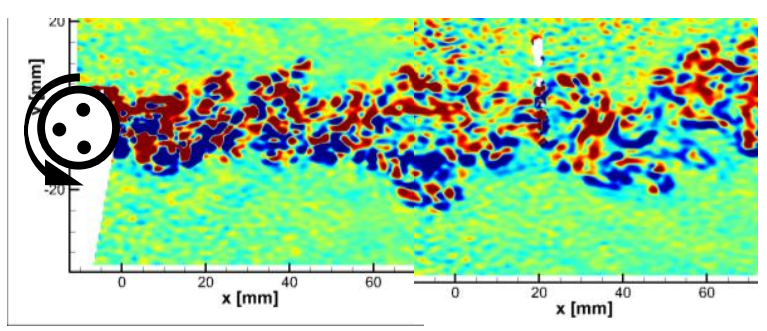

(d)

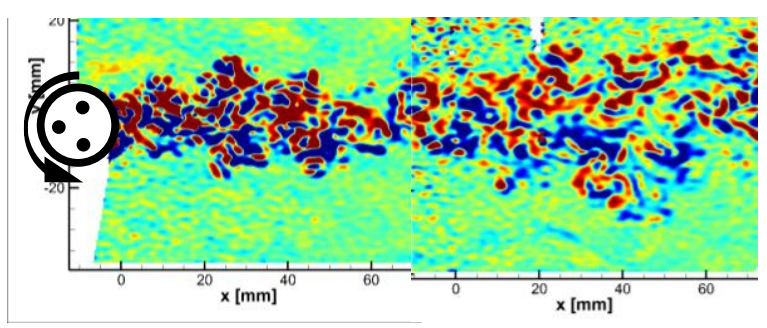

(f)

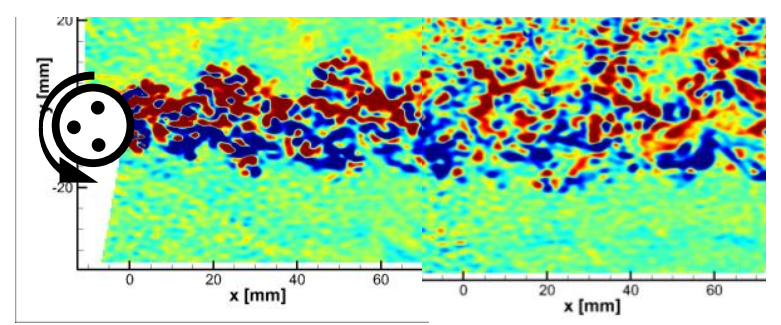

(g)

Figure 35. Instantaneous Vorticity Contour at Re=858 and Rotation Speed is (a) 120 (b) 360 (c) 960 (d) 1500 (e) 1800 (f) 2400 (g) 3000 RPM 


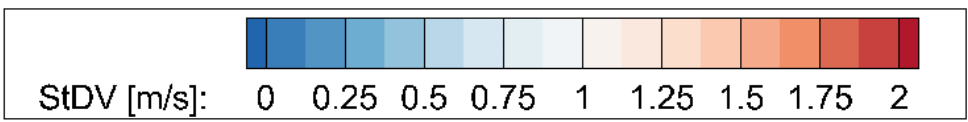

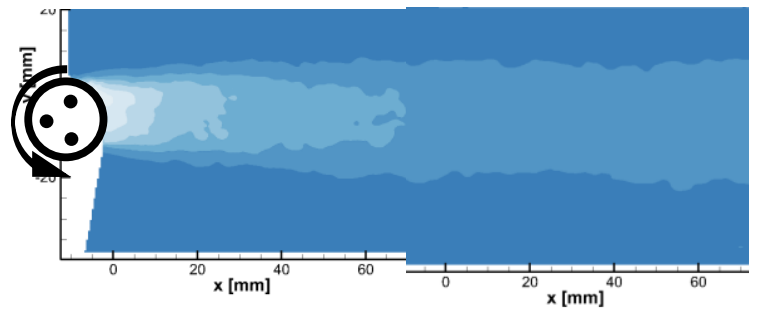

(a)

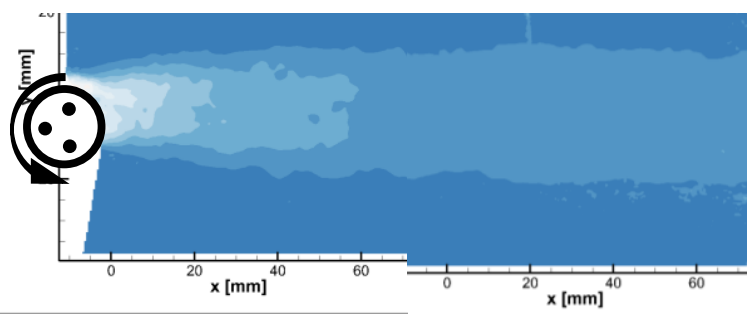

(b)

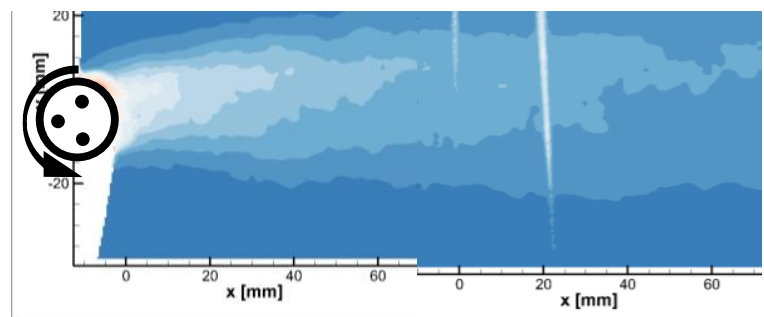

(e)

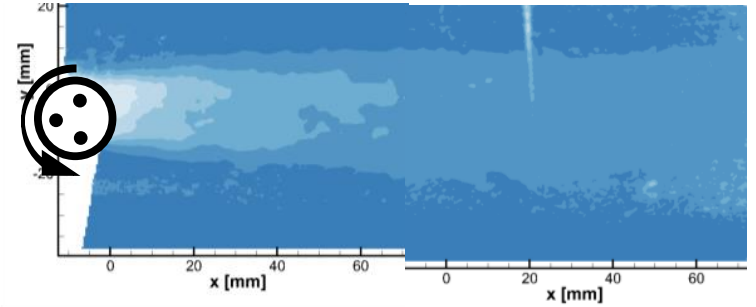

(b)

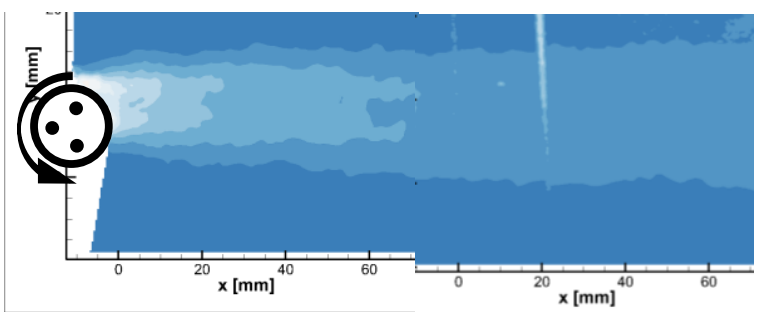

(d)

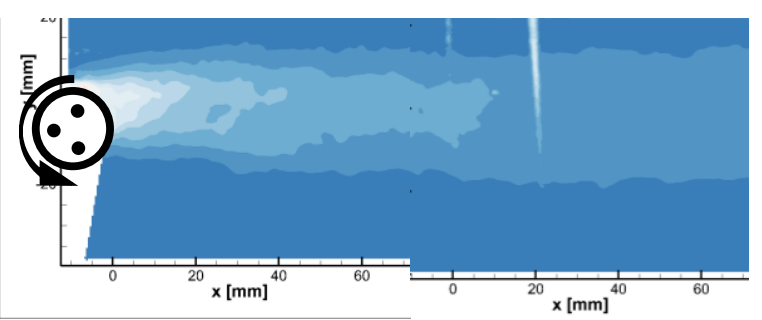

(f)

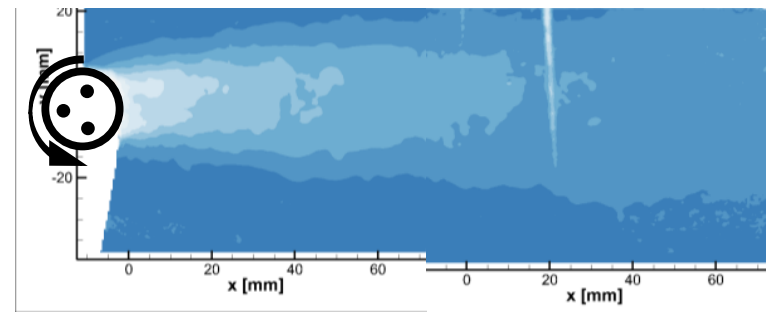

$(\mathrm{g})$

Figure 36. Standard Deviation at $\mathrm{Re}=57$ and Rotation Speed is (a) 120 (b) 360 (c) 960 (d) 1500 (e) 1800 (f) 2400 (g) 3000 RPM 


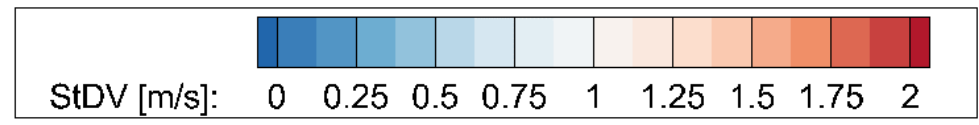

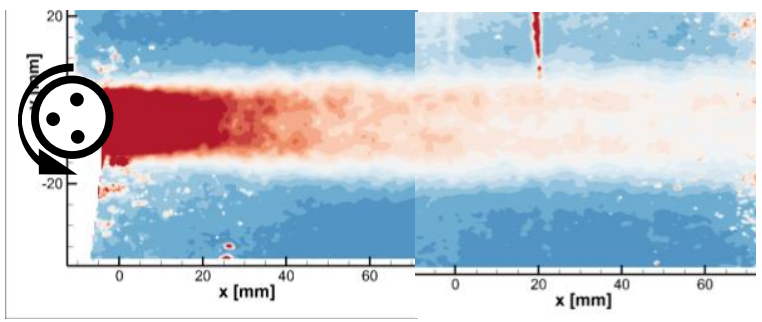

(a)

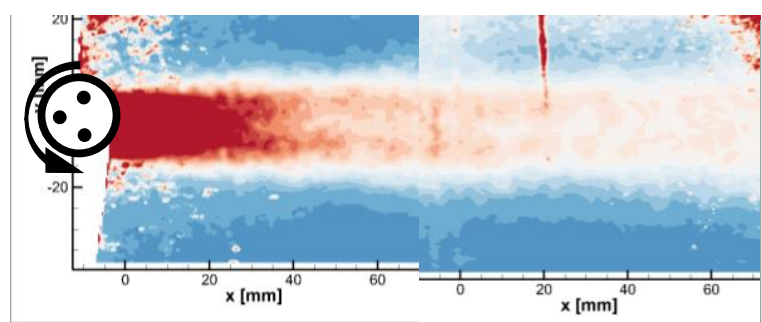

(c)

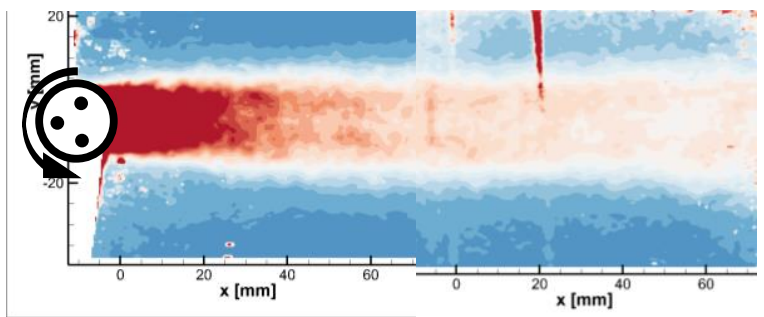

(e)

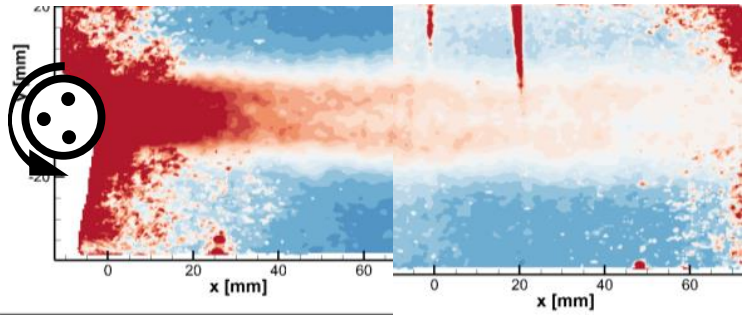

(b)

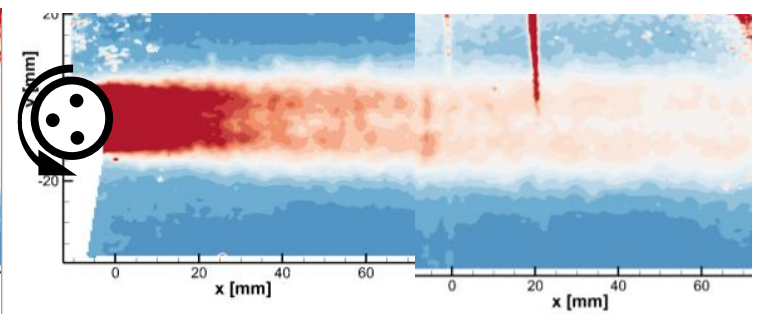

(d)

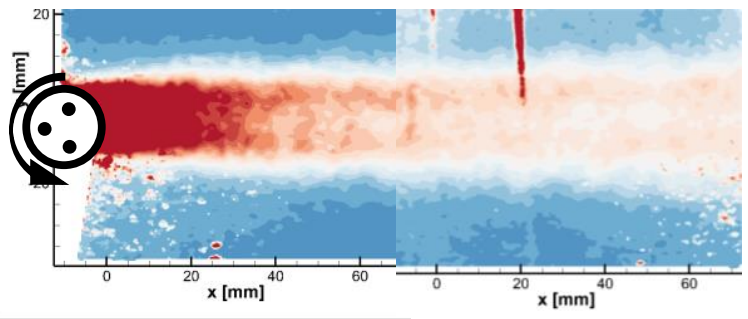

(f)

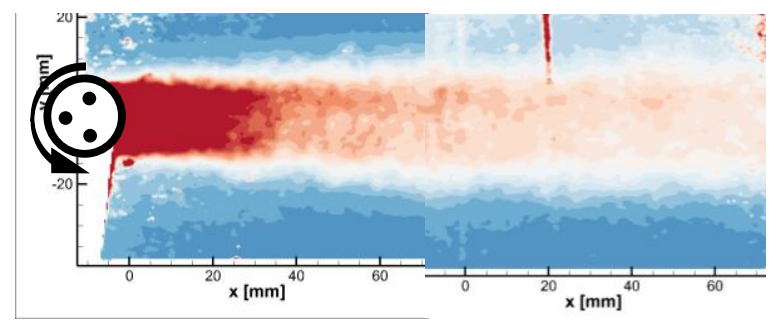

(g)

Figure 37. Standard deviation at $\mathrm{Re}=1700$ and Rotation Speed is (a) 120 (b) 360 (c) 960 (d) 1500 (e) 1800 (f) 2400 (g) 3000 RPM 
In Figure 38 and Figure 39, multiple instantaneous plot is shown when the cylinder orientation was $0^{\circ}$ and $180^{\circ}$ during the rotation at $\mathrm{Re}=57$ and $\mathrm{RPM}=120,360$. Higher RPM plots were shows in APPENDIX-G. It is important to note that all the instantaneous plots are time uncorrelated. For a specific incident angle during rotation, the results show that the flow is pattern is not phase locked. Most of the conditions show that the wake breaks into smaller positive and negative voritices due to rotation of cylinder system and theirs mixing evolves to a distinct and random vortex shadding at fixed angle of orientation and with varying RPM as well. However, at low RPM (For example, RPM=120 and 360), the vortex shedding in the downstream looks more organized than higher RPM. At RPM $=120$, when the cylinder orientation is $180^{\circ}$, some instances shows vortex shedding similar to single cylinder vortex shedding. As the RPM increases, the positive and negative vortices spread over the flow domain and they travel in the downstream as smaller vortices in unorganized manner. This phenomenon indicates that increase in RPM cause wake mixing that result in transition from laminar to turbulent flow. 


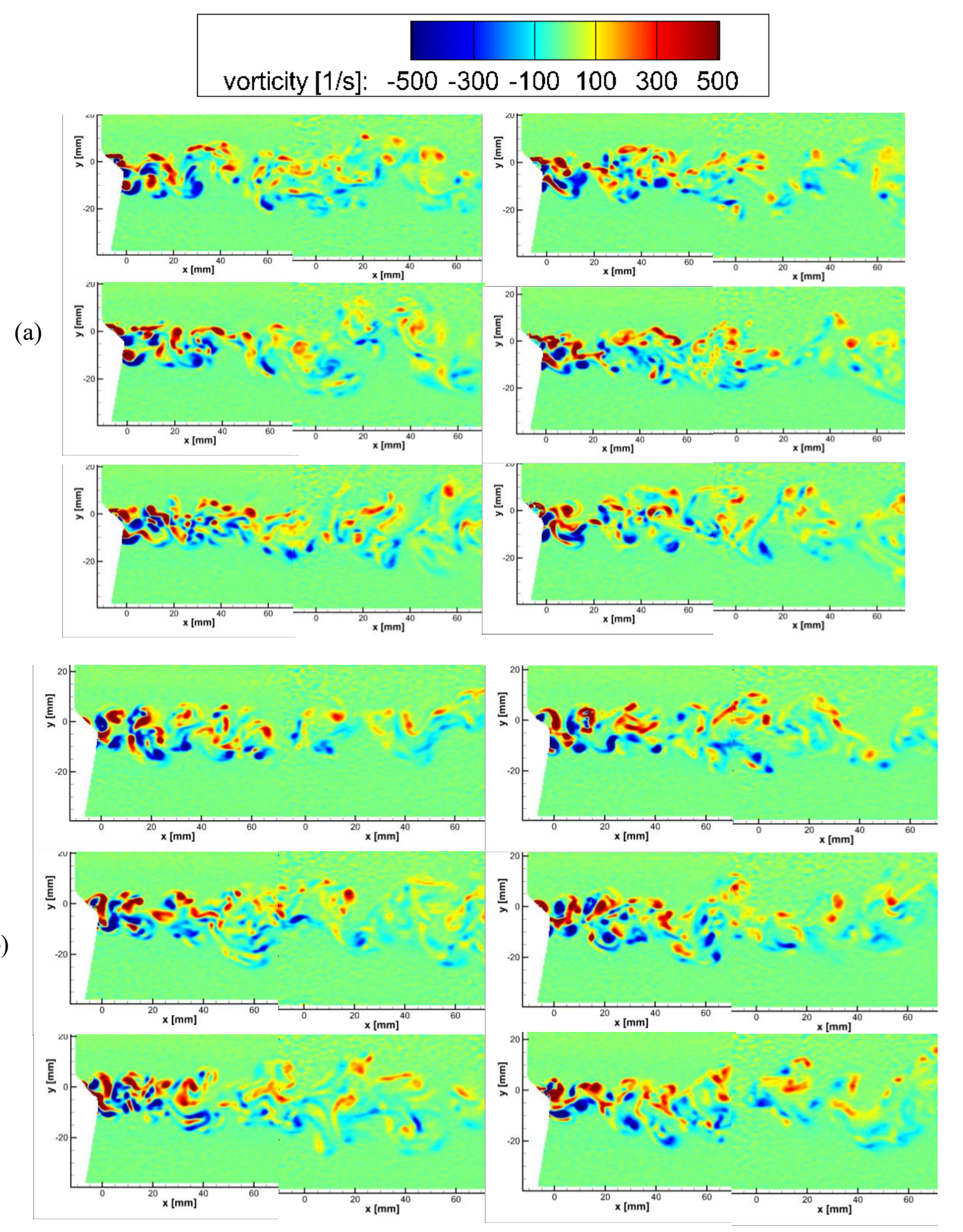

Figure 38. Instantaneous vorticity plot when the cylinder orientation was (a) $0^{\circ}$ (b) $180^{\circ}$ at $\operatorname{Re}=57$ rotation $\mathrm{RPM}=120 \mathrm{~Hz}$ 


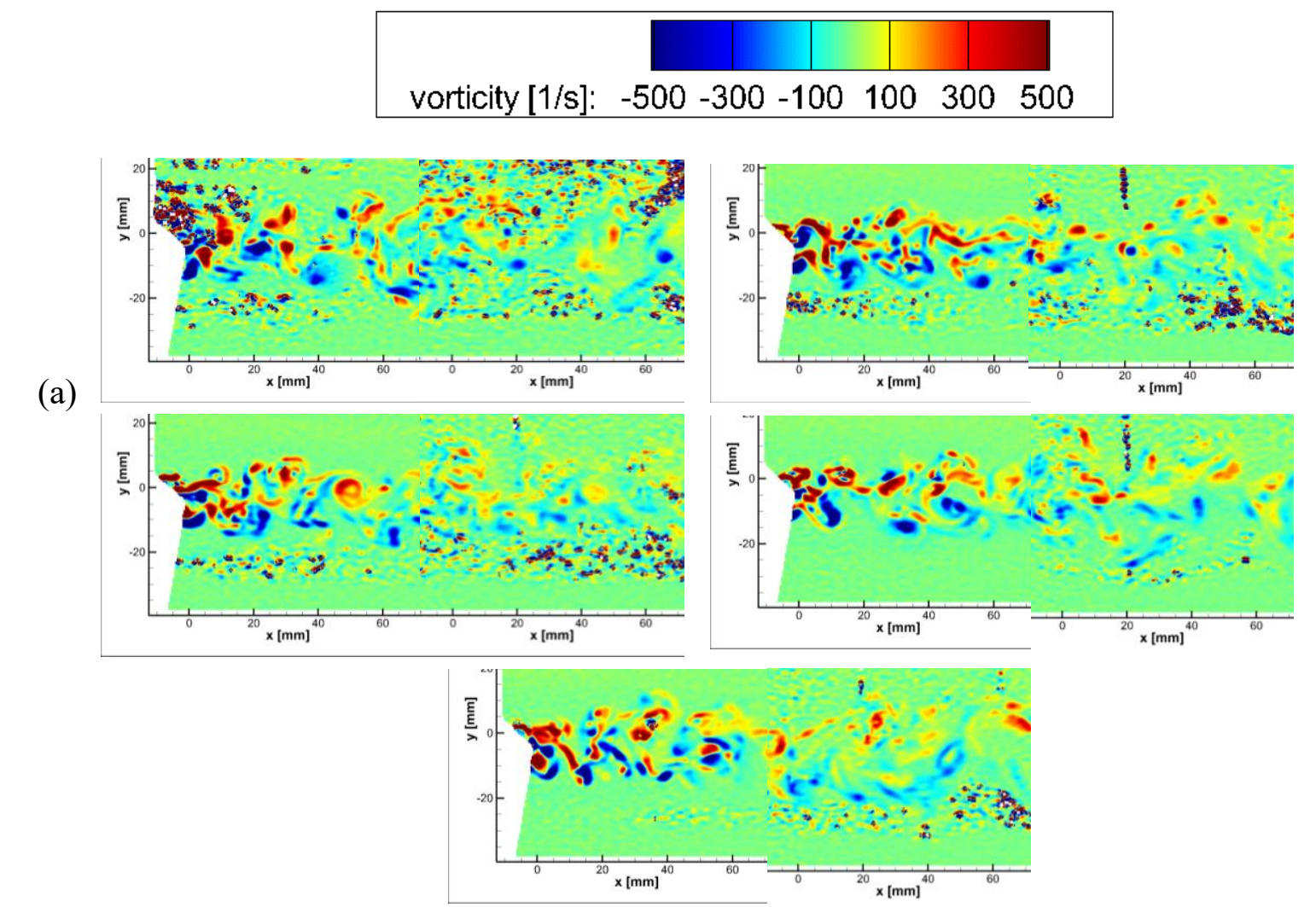

(b)
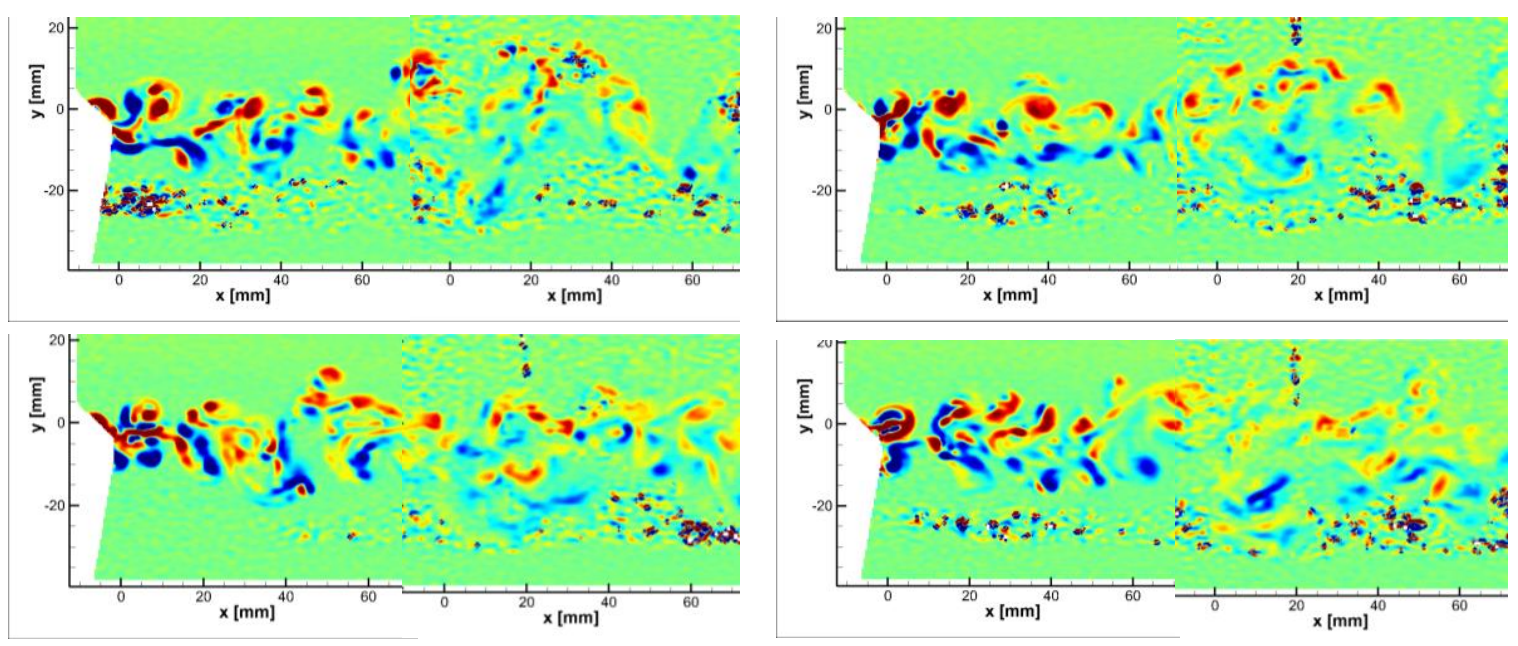

Figure 39. Instantaneous vorticity plot when the cylinder orientation was (a) $0^{0}$ (b) $180^{0}$ at $\operatorname{Re}=57$ rotation $\mathrm{RPM}=360 \mathrm{~Hz}$ 


\section{Particle Image Velocimetry (Close View)}

In the second campaign of PIV, the data's were taken with very close view of camera. Two camera was used to obtain the data at static and rotating condition. With close view of PIV, the data will help to get the detail structure of wake in the downstream of cylinder. At this stage, the data's were taken for both static and rotating condition when the Re number varies from 37 to 1700. For rotating condition, RPM was varied from 120 to 3000 for each Re number.

\section{$\underline{\text { Non-Rotating Cases: Flow Visualization }}$}

Figure 40 and Figure 41 shows the flow visualization at $\operatorname{Re}=37$ for $0^{0}$ and $180^{\circ}$ orientation of cylinder system. The flow visualization indicates the different structure of wake due to the change of orientation. The image clearly shows the cylinder wake interaction and later evolves to distinct vortex shedding which is unique and different than individual cylinder vortex shedding. At both orientation, all five instances proves that the downstream cylinder wake dominates. When the orientation is $0^{0}$, the wake from upstream cylinder combines and evolves to round vortices in downstream. As the cylinder orientation changes to $180^{\circ}$, the presence of downstream cylinder makes the upstream wake from pair of cylinder narrow. This pattern was also observed in our first PIV campaign at $\mathrm{Re}=57$. 

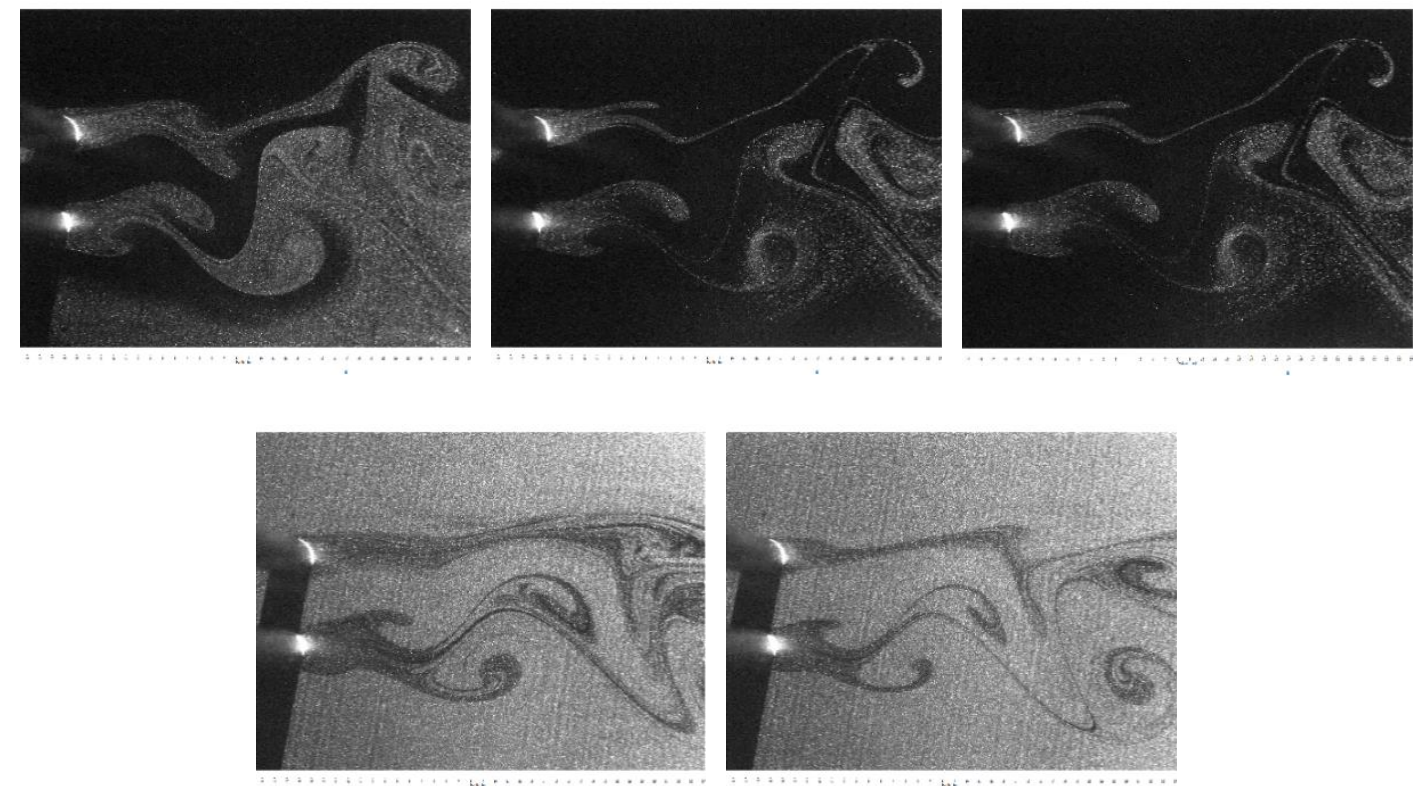

Figure 40. Flow visualization at $\mathrm{Re}=37$ and Static Angle $0^{0}$
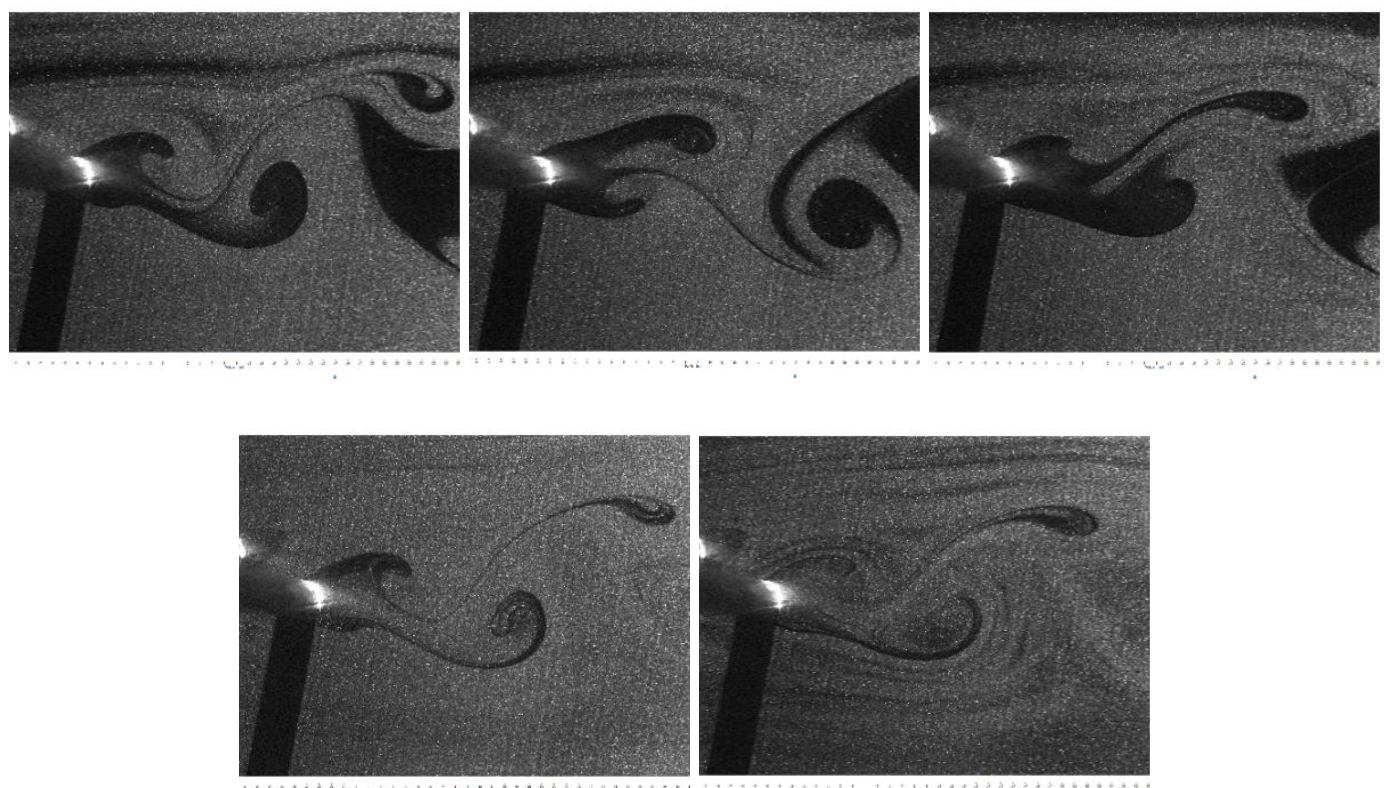

Figure 41. Flow visualization at $\mathrm{Re}=37$ and Static Angle $180^{\circ}$ 


\section{Non-Rotating Cases: Instantaneous and Average Velocity Contour}

Figure 42 and Figure 43 shows the velocity and vorticity contour map at three different orientation at $\operatorname{Re}=37$. The resuts shows that how the wake shape changes as the orientation changes. The close view of vector plot shows more detail picture of the wake coming out of individual cylinder. As the Re number increases the wake structure for individual cases changes. This phenomena can be observed in the instantaneous plots shown in Figure 44 to Figure 48. The average velocity was calculated based on 100 instantaneous time uncorelated velocity contour. With the change Re, the average velocity plot does not show any significant different for specific orienation of cylinder arrangement. However, for fixed Re number the average velocity profile shows distinct difference as the orientation changes. 


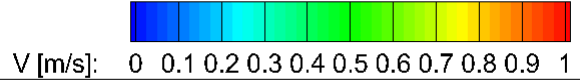

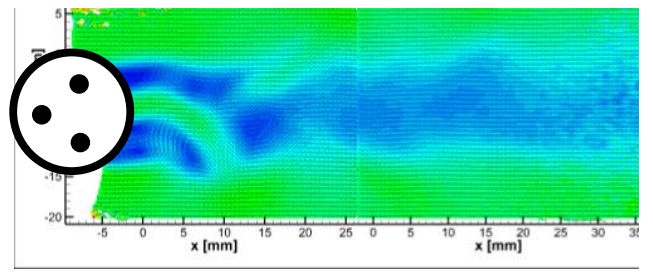

(a)

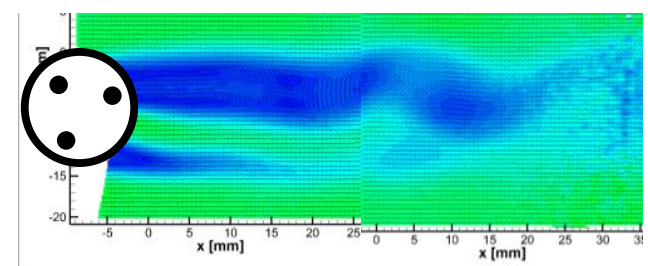

(b)

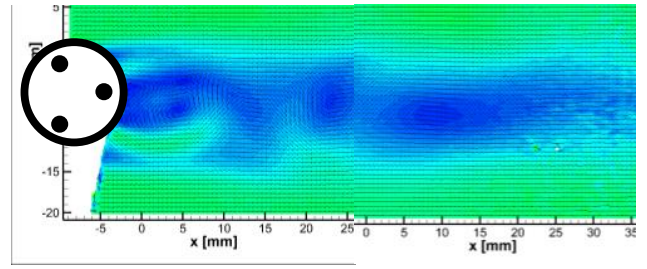

(c)

Figure 42. Instantaneous velocity contour at $\mathrm{Re}=37$ and incident angle (a) $0^{0}$ (b) $45^{\circ}$ and (c) $90^{\circ}$

vorticity [1/s]: $\quad-500-400 \quad-300-200-100 \quad 0 \quad 100 \quad 200 \quad 300 \quad 400 \quad 500$

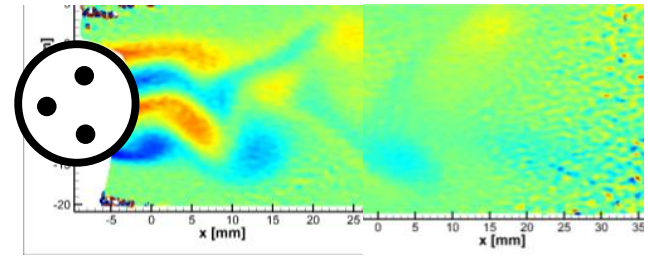

(a)

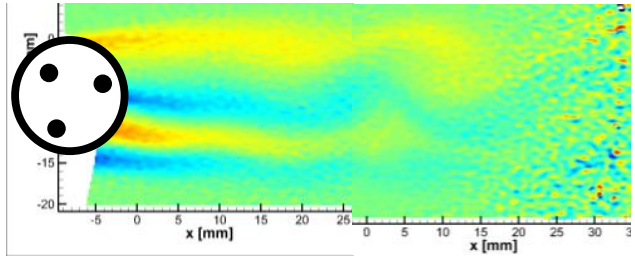

(b)

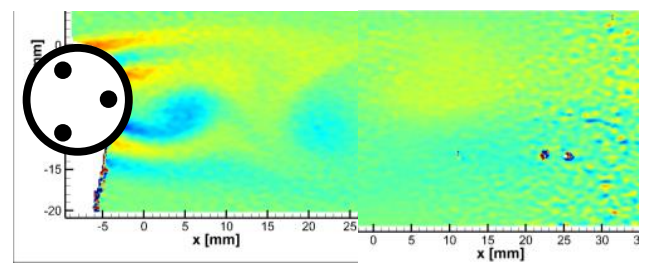

(c)

Figure 43. Instantaneous vorticity contour at $\mathrm{Re}=37$ and incident angle (a) $0^{\circ}$ (b) $45^{\circ}$ and (c) $90^{\circ}$ 


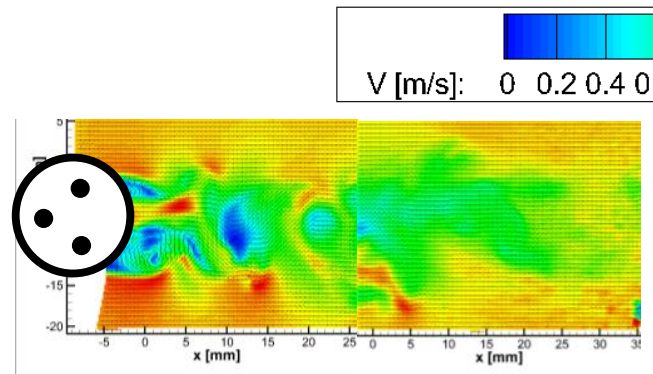

(a)

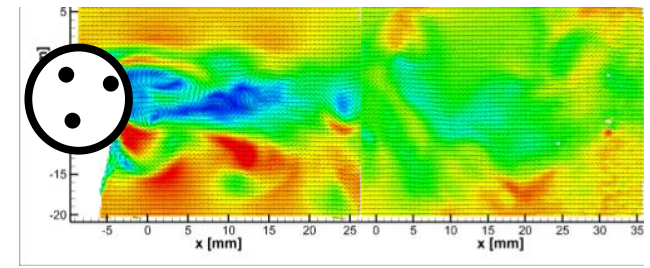

(b)

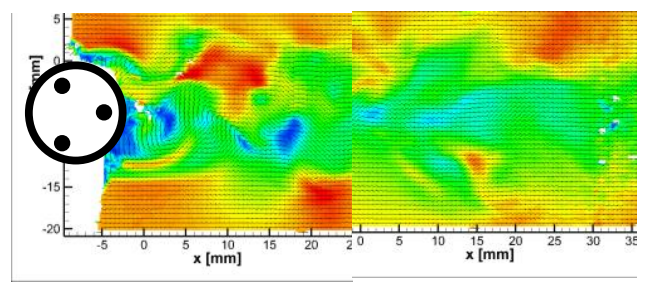

(c)

Figure 44. Instantaneous velocity contour at $\mathrm{Re}=57$ and incident angle (a) $0^{\circ}$ (b) $45^{\circ}$ and (c) $90^{\circ}$

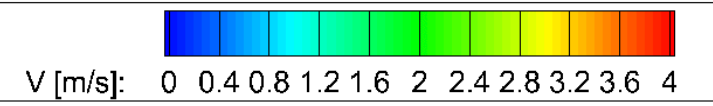

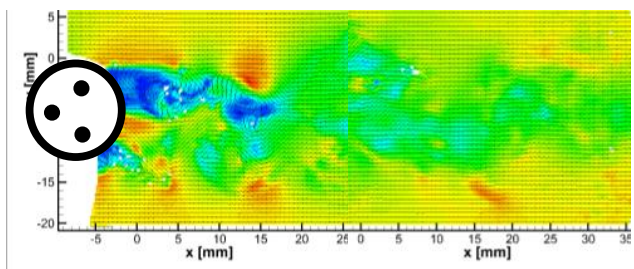

(a)

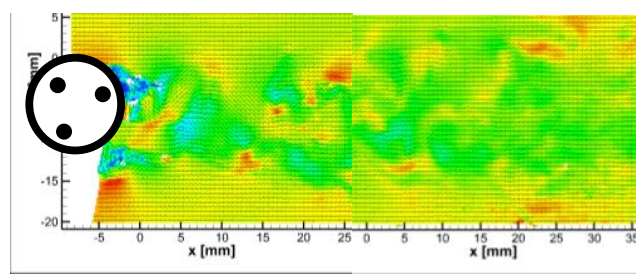

(b)

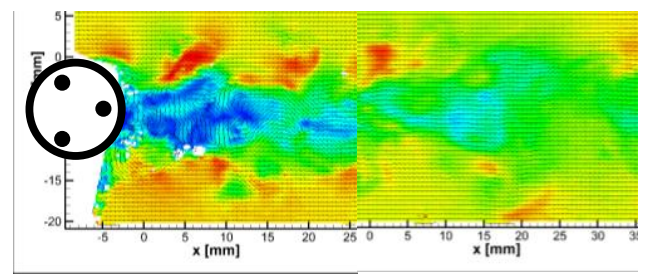

(c)

Figure 45. Instantaneous velocity contour at $\mathrm{Re}=500$ and incident angle (a) $0^{\circ}$ (b) $45^{\circ}$ and (c) $90^{\circ}$ 


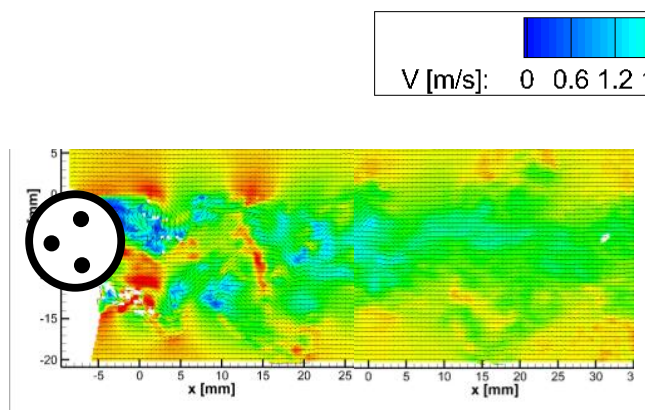

(a)

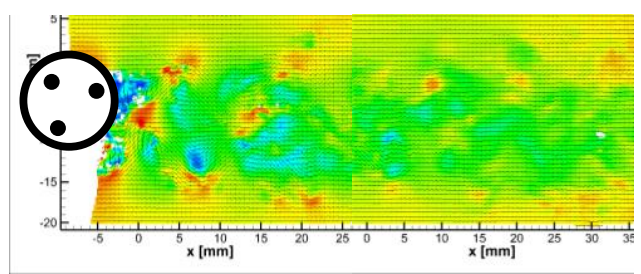

(b)

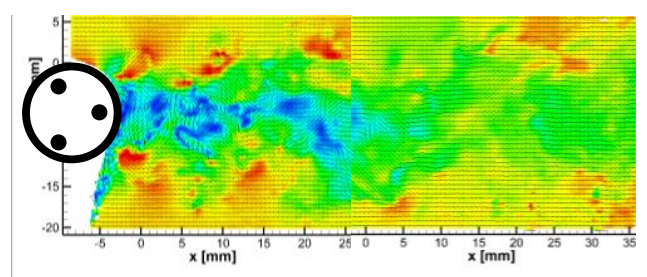

(c)

Figure 46. Instantaneous velocity contour at $\mathrm{Re}=858$ and incident angle (a) $0^{\circ}$ (b) $45^{\circ}$ and (c) $90^{\circ}$

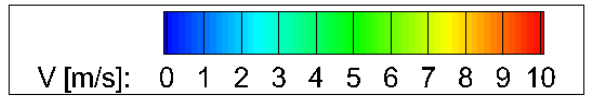

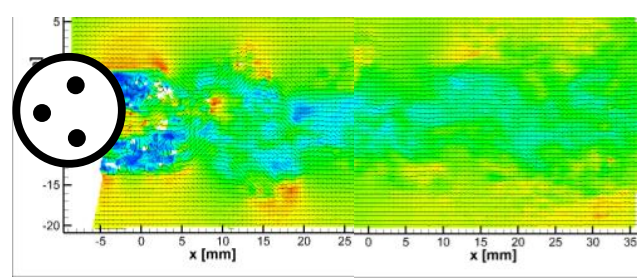

(a)

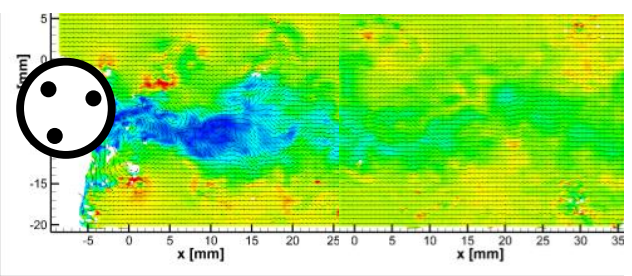

(b)

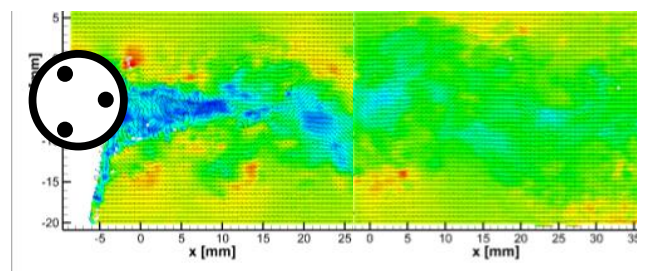

(c)

Figure 47. Instantaneous velocity contour at $\mathrm{Re}=1250$ and incident angle (a) $0^{\circ}$ (b) $45^{\circ}$ and (c) $90^{\circ}$ 


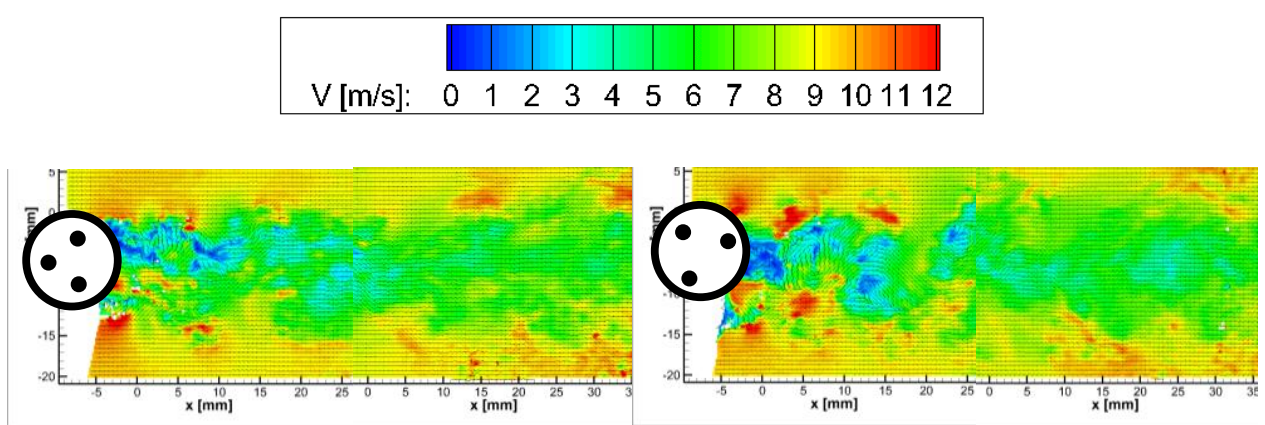

(a)

(b)

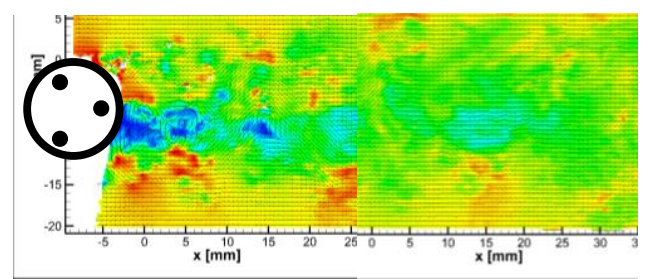

(c)

Figure 48. Instantaneous velocity contour at $\mathrm{Re}=1700$ and incident angle (a) $0^{\circ}$ (b) $45^{\circ}$ and (c) $90^{0}$

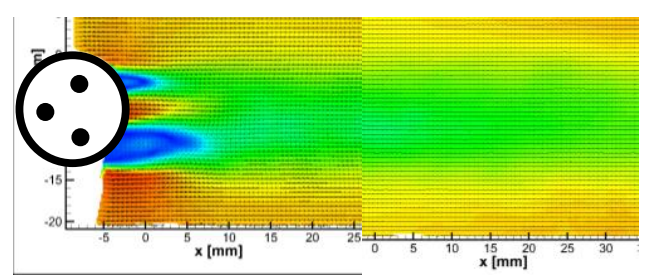

(a)

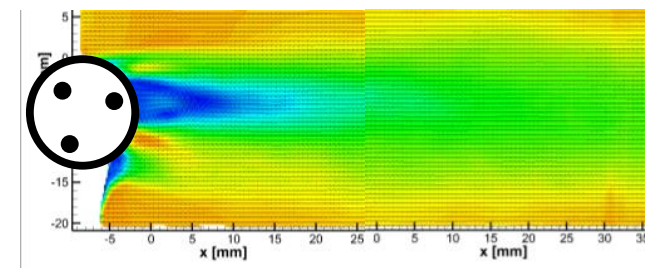

(b)

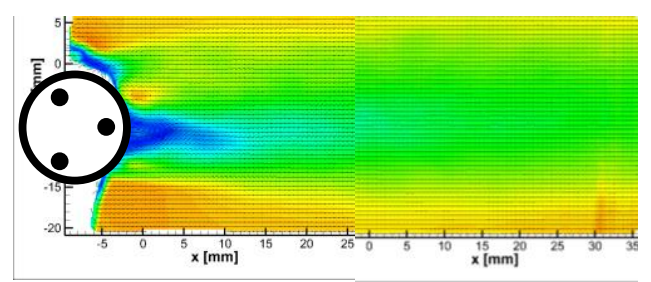

(c)

Figure 49. Average velocity contour at $\mathrm{Re}=57$ and incident angle (a) $0^{\circ}$ (b) $45^{\circ}$ and (c) $90^{\circ}$ 


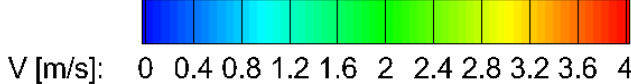

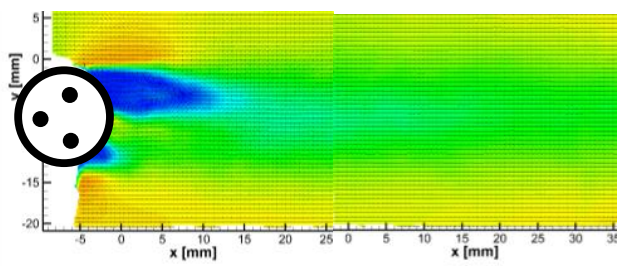

(a)

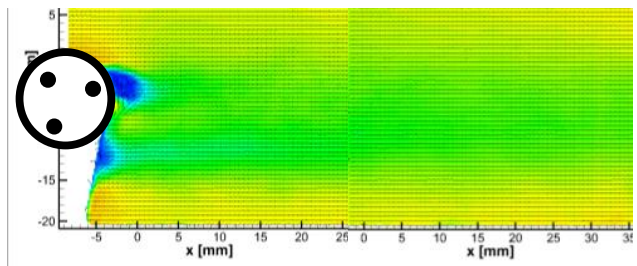

(b)

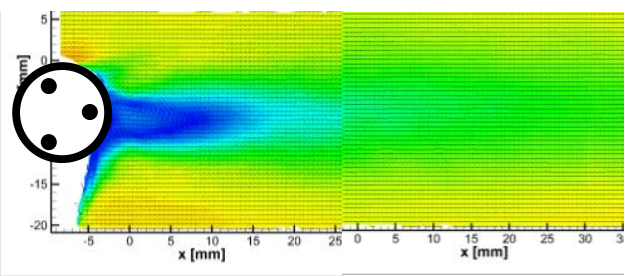

(c)

Figure 50. Average velocity contour at $\mathrm{Re}=500$ and incident angle (a) $0^{0}$ (b) $45^{\circ}$ and (c) $90^{\circ}$

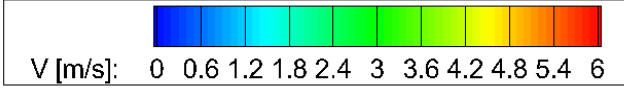

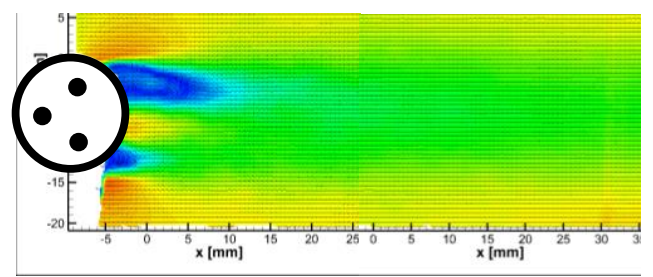

(a)

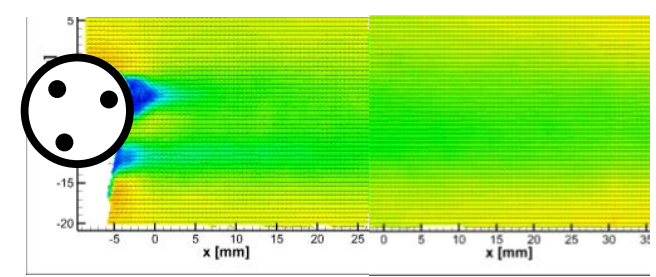

(b)

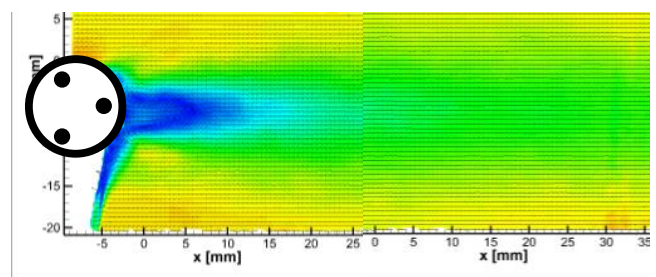

(c)

Figure 51. Average velocity contour at $\mathrm{Re}=858$ and incident angle (a) $0^{0}$ (b) $45^{\circ}$ and (c) $90^{\circ}$ 


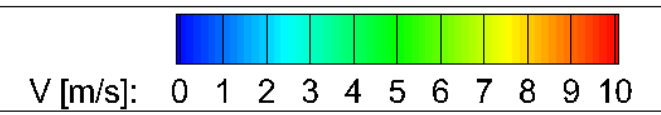

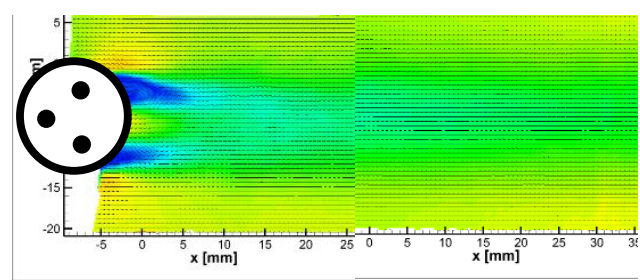

(a)

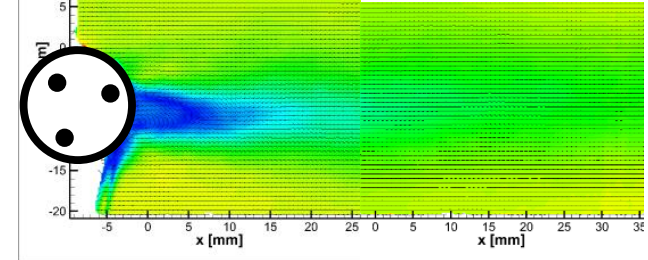

(b)

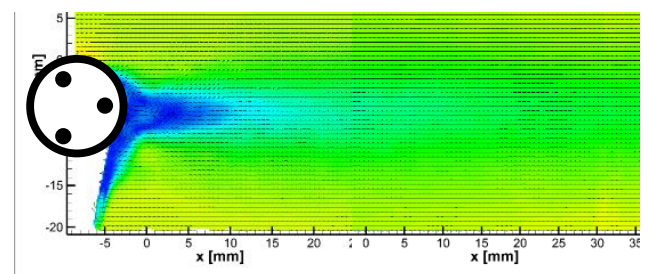

(c)

Figure 52. Average velocity contour at $\mathrm{Re}=1250$ and incident angle (a) $0^{\circ}$ (b) $45^{\circ}$ and (c) $90^{\circ}$

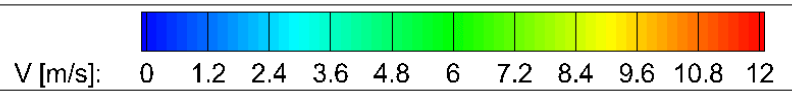

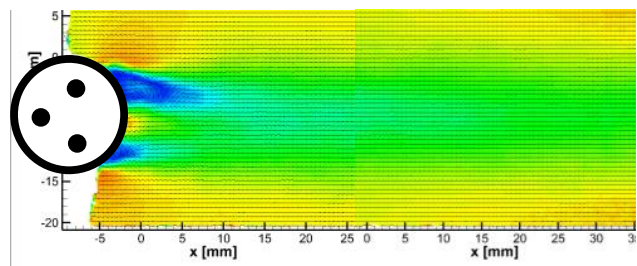

(a)

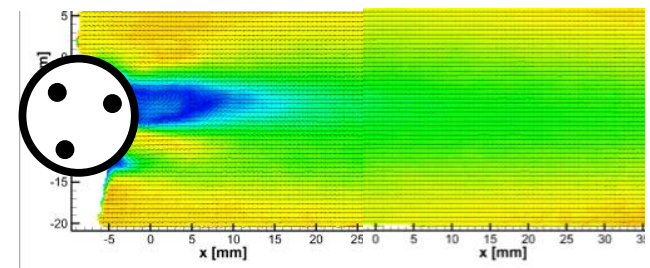

(b)

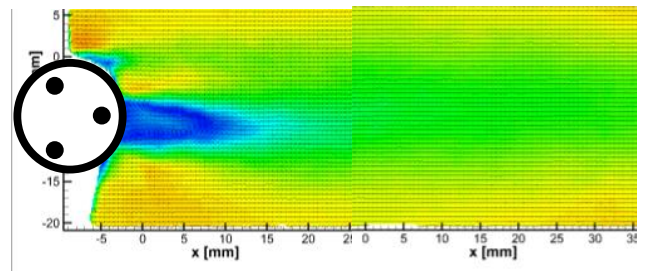

(c)

Figure 53. Average velocity contour at $\mathrm{Re}=1700$ and incident angle (a) $0^{0}$ (b) $45^{\circ}$ and (c) $90^{\circ}$ 


\section{Rotating Cases: Insantaneous Velocity and Vorticty Contour}

The close view of velocity contour, Figure 54 and in APPENDIX-H, when the cyinder is rotating shows significant detail stucture which we were unable to capture from far view. When the Re number is 57, in Figure 54, the flow structure in the downstream changes due to increase in RPM. At such low Re number, the RPM of the cylinder creates recirculation region very close to cylidner system. As the RPM increases very high, flow instability arises due to the motion of cylinder with and against the flow at same time. After observing 100 of instantaneous cases for each condition, the results indicate that the flow is very unstready and turbulent due to the wake interation and Rotation of the cylinder. Figure 55 shows the vorticity contour plot at $\mathrm{Re}=57$. As the Reynolds number increase the vorticity field becomes very noisy. It becomes difficult to extract useful information with high accumalation of noise from the vorticity contour. That's why we chose to show only voriticity contour at low Re number. The vorticity results obtained from the second campaign of PIV experiment is also consistant with our first PIV experiment. The results shows due to the rotation of cylinder the wake from the individual cylinder breaks down to smaller positive and negative vorticies. Their interation results in distinct vortex shedding.

As the Re number increases to $500,858,1250$, and 1700 , the results show that the recirculation region starts to get smaller. This might happen as the convective velocity starts to dominate the flow. At such Re number the flow becomes more unsteady and turbulent. 


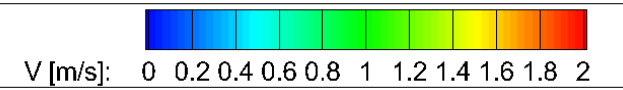

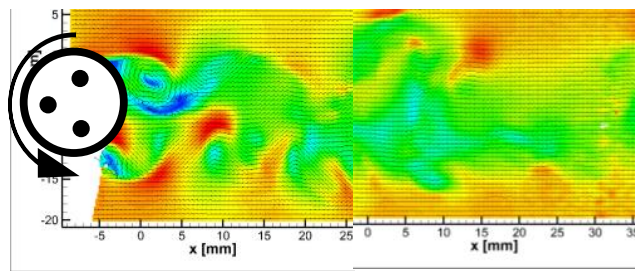

(a)

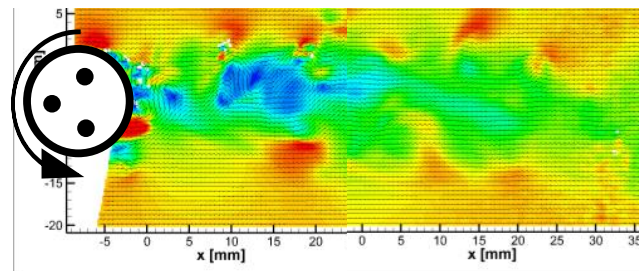

(c)

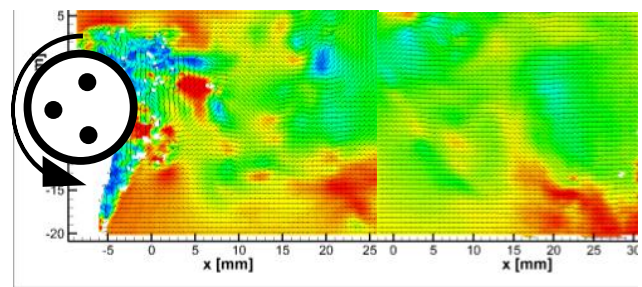

(e)

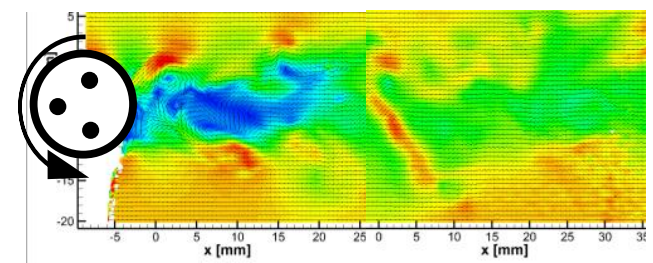

(b)

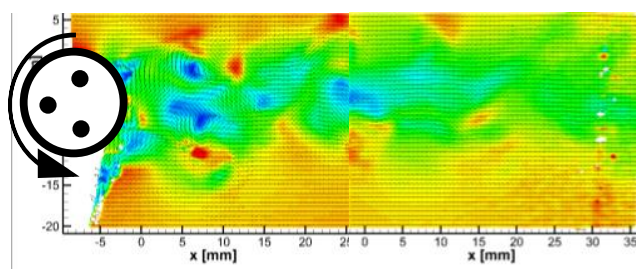

(d)

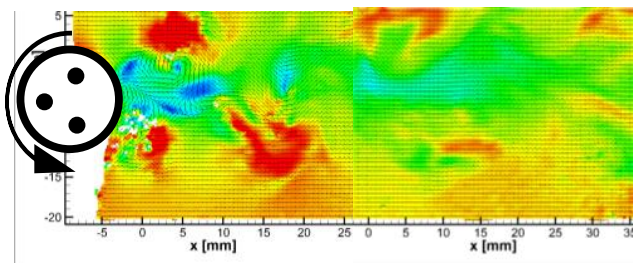

(f)

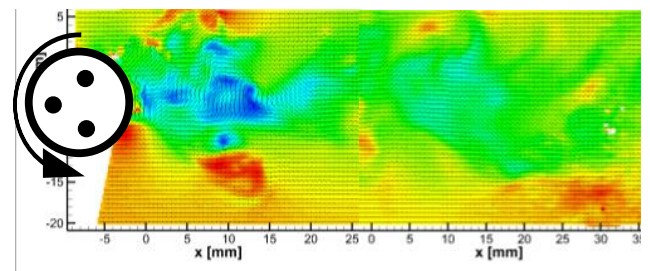

$(\mathrm{g})$

Figure 54. Instantaneous Velocity Contour at $\mathrm{Re}=57$ and Rotation Speed is (a) 120 (b) 360 (c) 960 (d) 1500 (e) 1800 (f) 2400 (g) 3000 RPM 


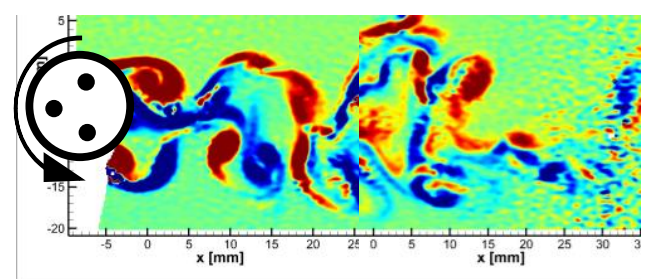

(a)

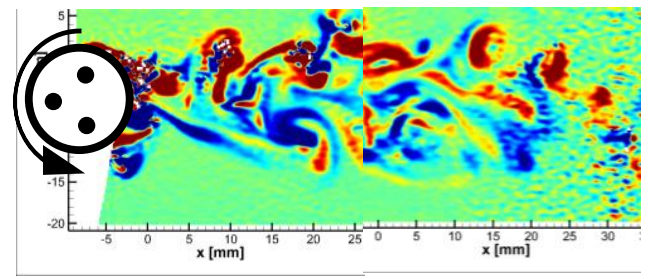

(c)

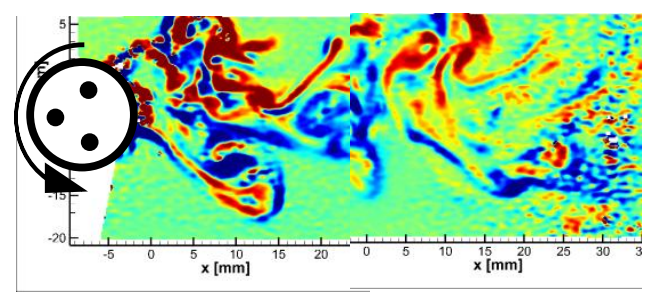

(e)

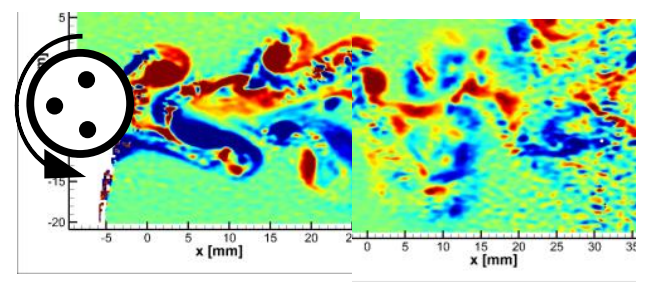

(b)

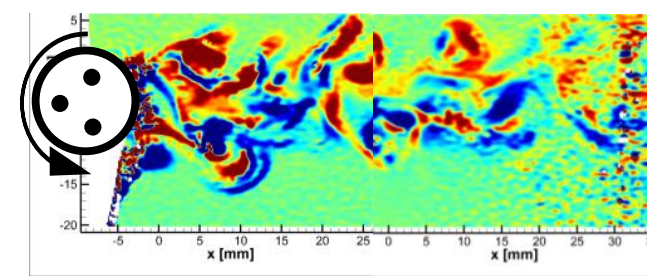

(d)

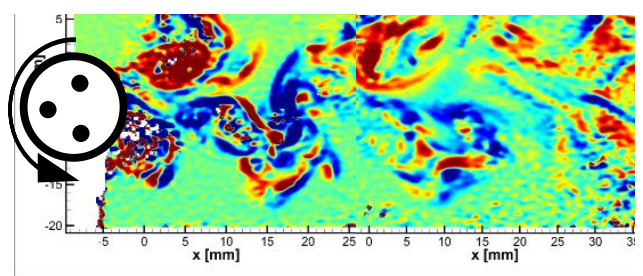

(f)

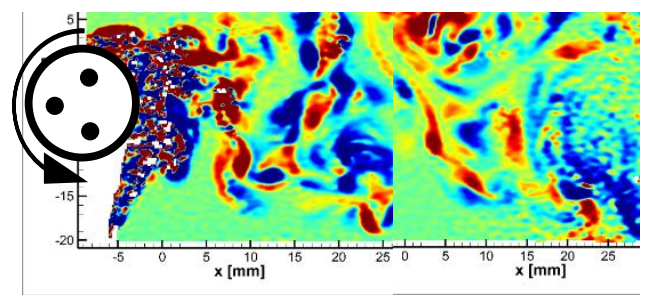

(g)

Figure 55. Instantaneous Vorticity Contour at $\mathrm{Re}=57$ and Rotation Speed is (a) 120 (b) 360 (c) 960 (d) 1500 (e) 1800 (f) 2400 (g) 3000 RPM 


\section{Tomography Results}

\section{$\underline{\text { Non-Rotating Case at } \mathrm{Re}=37}$}

The tomography experiment provide us the information of flow in three dimensional $\mathrm{X}, \mathrm{Y}$, and $\mathrm{Z}$ axis. Figure 56 to Figure 58 shows the vorticity contour at very low Re number at Static condition of $0^{\circ}, 45^{\circ}$, and $180^{\circ}$. For each of the case four different instances is shown. The vorticity contour for each conditions are shown at $\mathrm{Z}=-2.5,0$, and $2.5 \mathrm{~mm}$. The tomography results are consistent with PIV results. Each plots show that that the orientation of cylinder arrangement changes the vortex shedding structure. However, the vortex structure does not show any significant change along $\mathrm{Z}$ axis. This is very much expected as the tomography experiment was done with $6 \mathrm{~mm}$ thick LASER. At such low Re number, the flow structures are expected to remain very consistent. However, significant flow structure variation can be observed along the $\mathrm{x}$ direction.

Figure 59 to Figure 61 shows the iso surface of z-vorticity. These iso-sruface was plotted with negative 100 and positive 100 magnitude. Iso surface plots for each condition are time uncorrelated, but they provide significant detail how the vortex shedding looks like in threedimensional space. From the four instances for each condition, the results indicate that flow is phase locked for $0^{0}$ angle of orientation. $180^{\circ}$-angle orientation shows less phase lock characteristics then $0^{0}$ angle orientation whereas the $45^{\circ}$ does not show such characteristics because of asymmetric arrangement. 


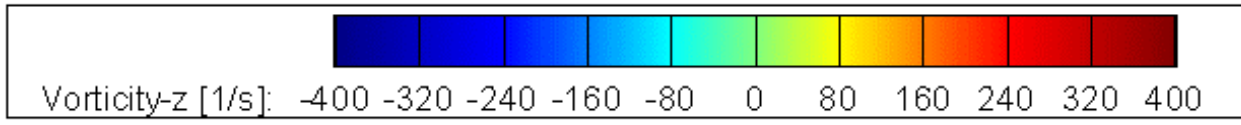

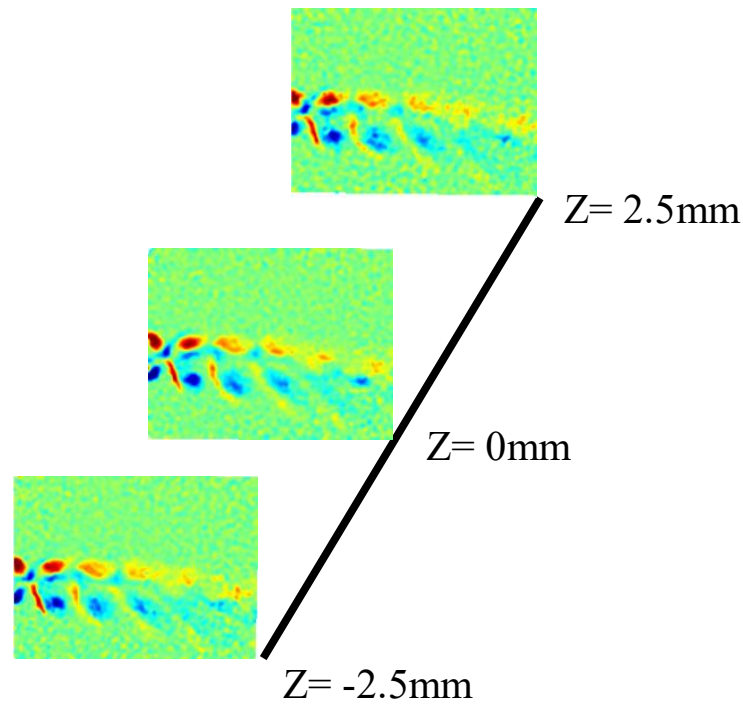

(a)

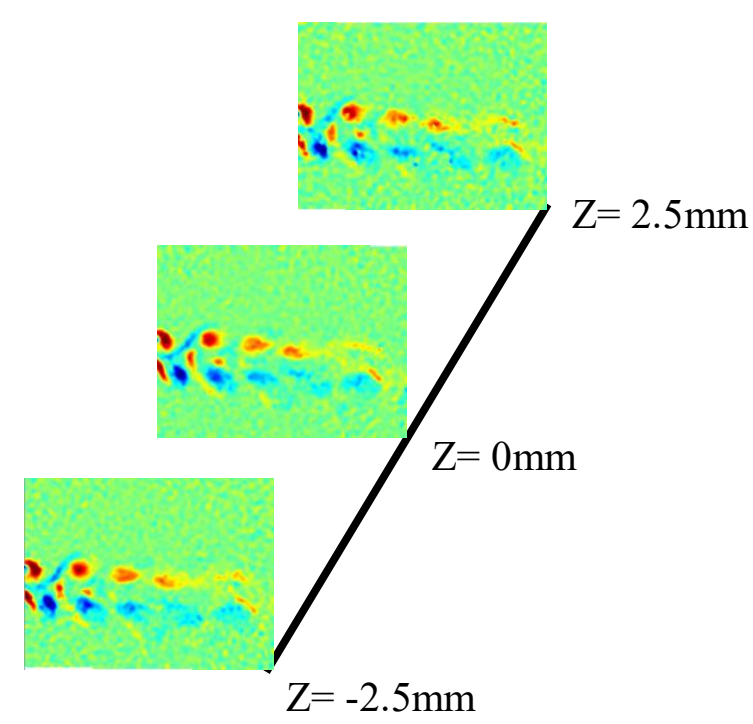

(c)

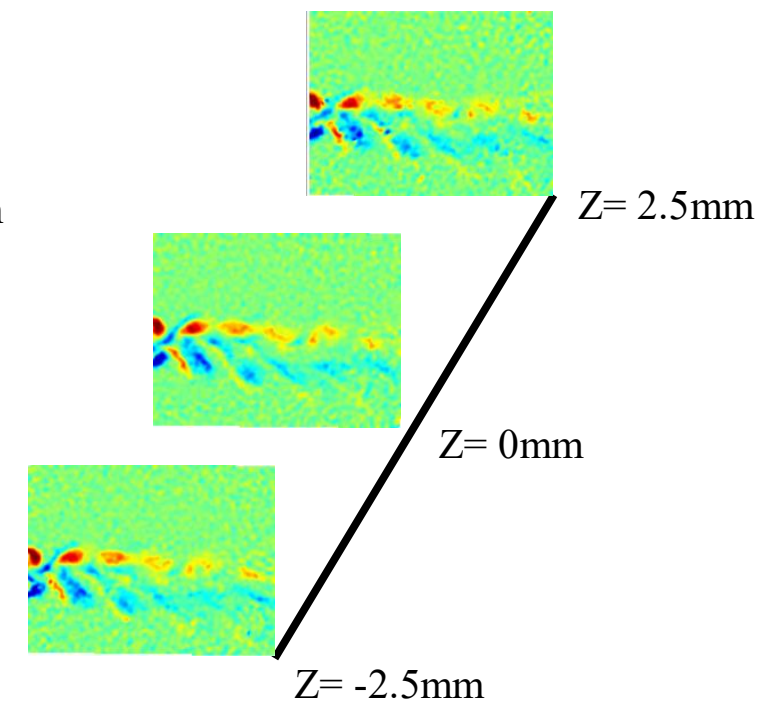

(b)

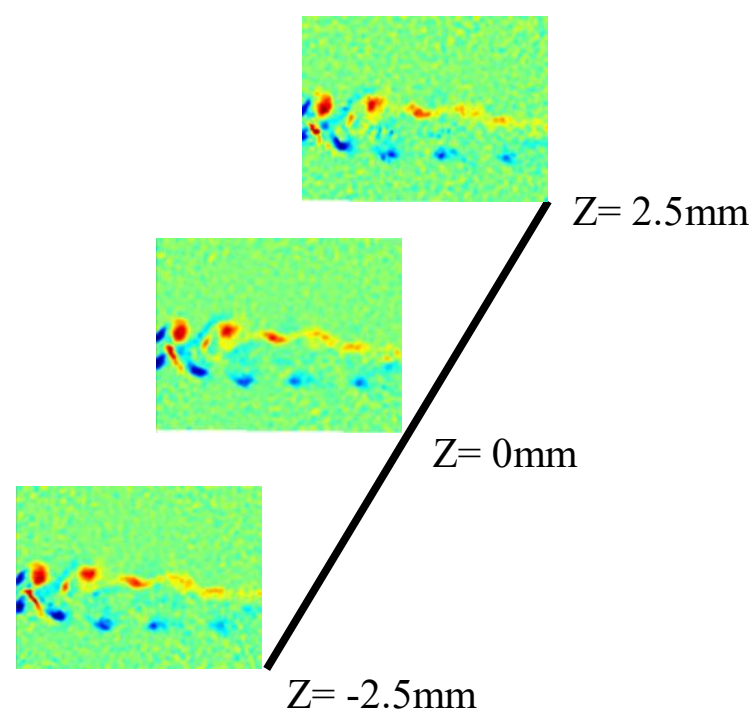

(d)

Figure 56. Vorticity contour at $\mathrm{Z}=-2.5,0$, and $2.5 \mathrm{~mm}$ for $\mathrm{Re}=37$ at incident angle $0^{0}$ 


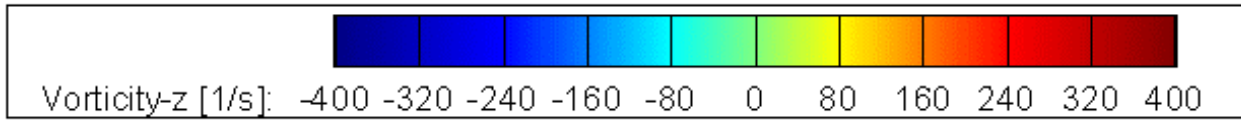

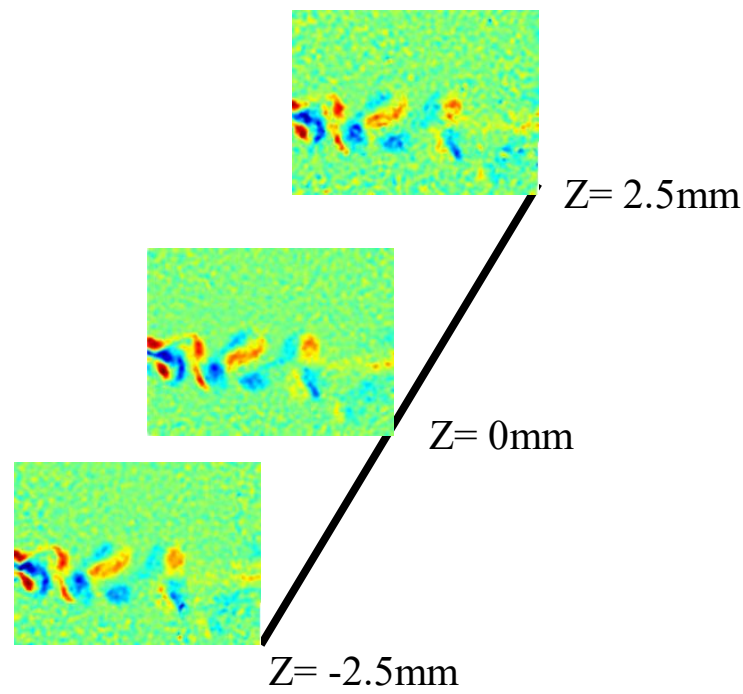

(a)

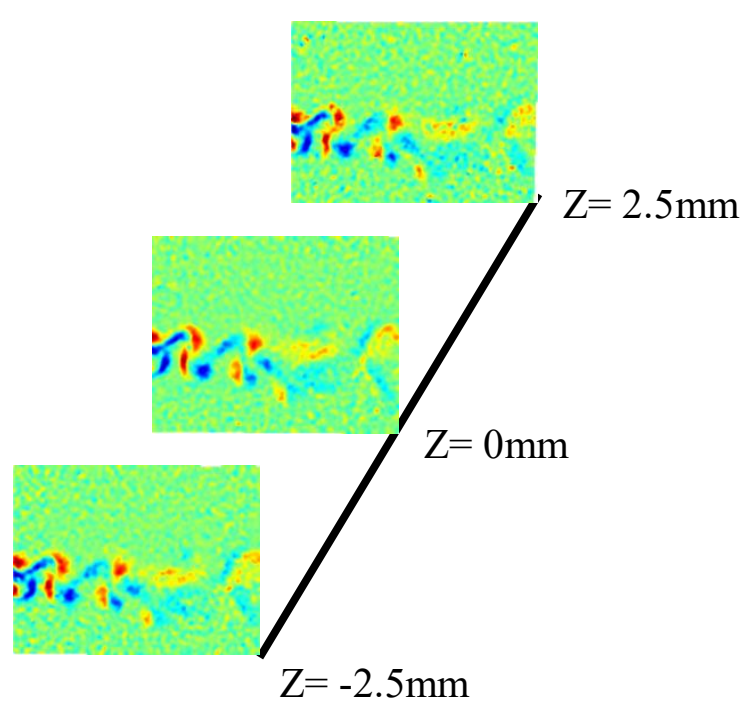

(c)

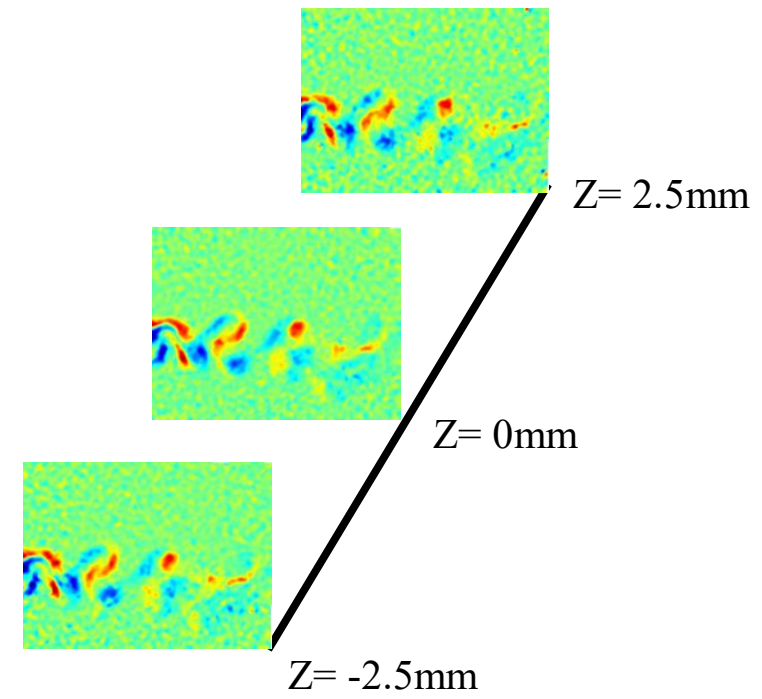

(b)

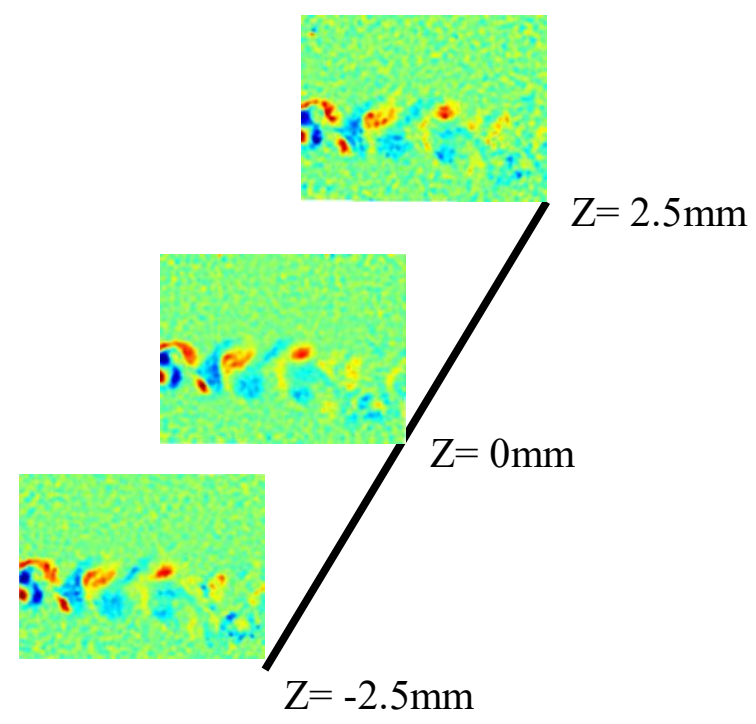

(d)

Figure 57. Vorticity contour at $Z=-2.5,0$, and $2.5 \mathrm{~mm}$ for $\mathrm{Re}=37$ at incident angle $45^{\circ}$ 


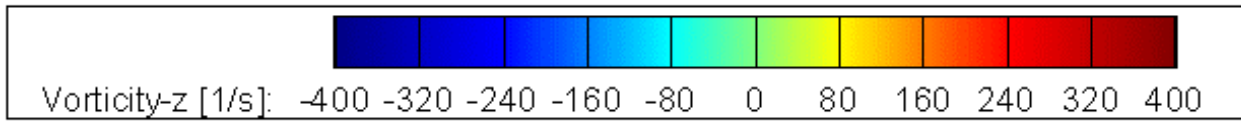

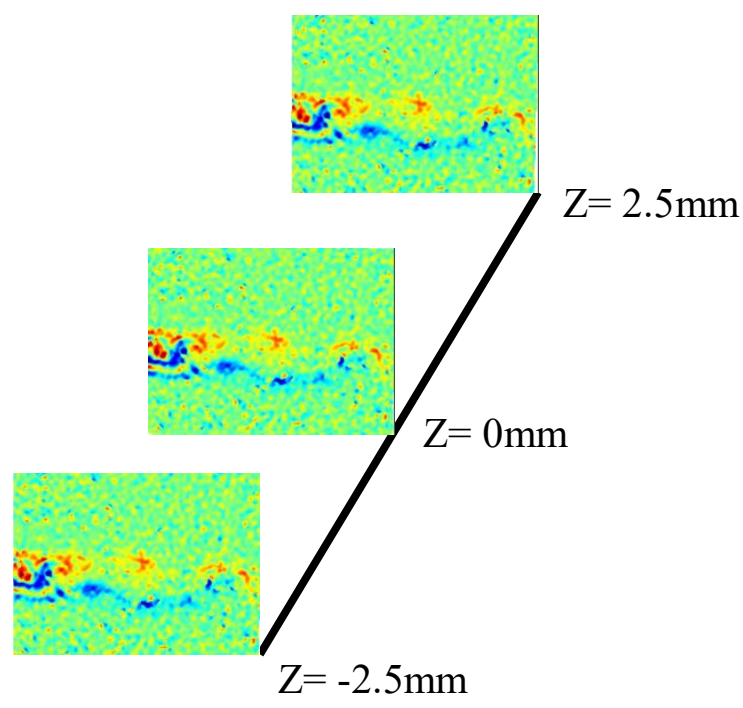

(a)

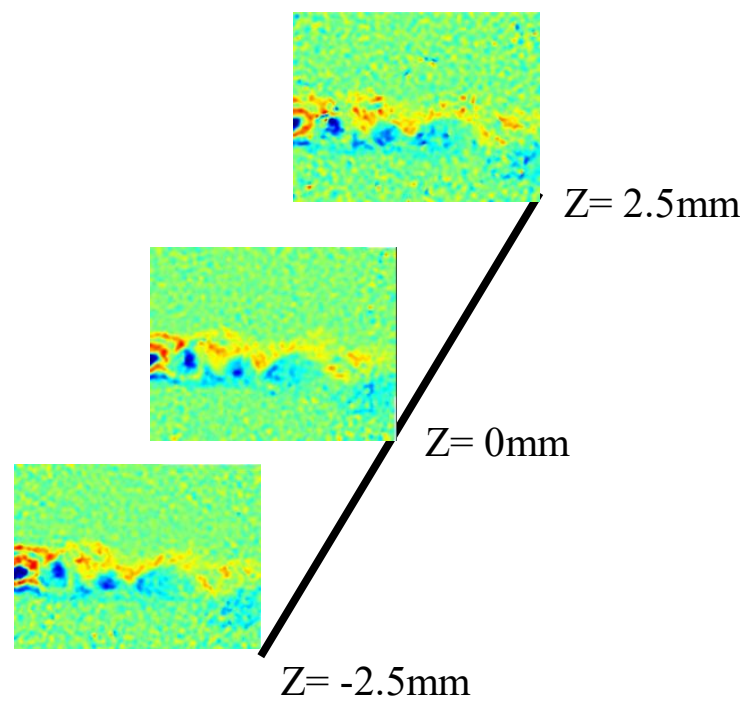

(c)

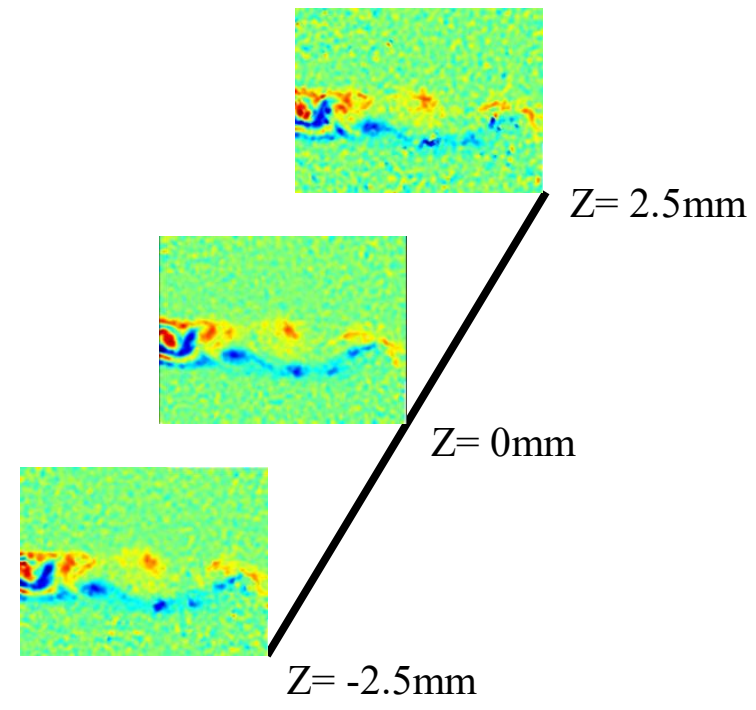

(b)

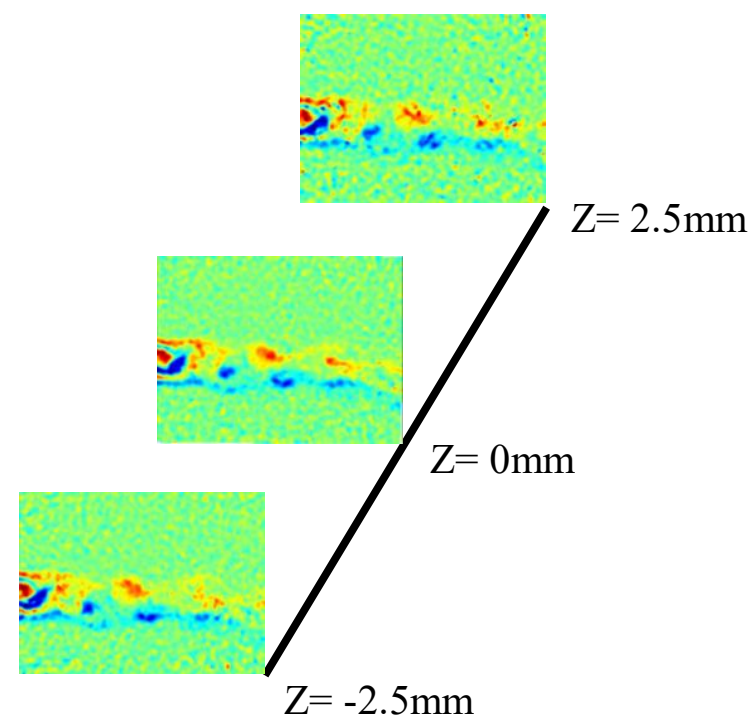

(d)

Figure 58. Vorticity contour at $\mathrm{Z}=-2.5,0$, and $2.5 \mathrm{~mm}$ for $\mathrm{Re}=37$ at incident angle $180^{\circ}$ 

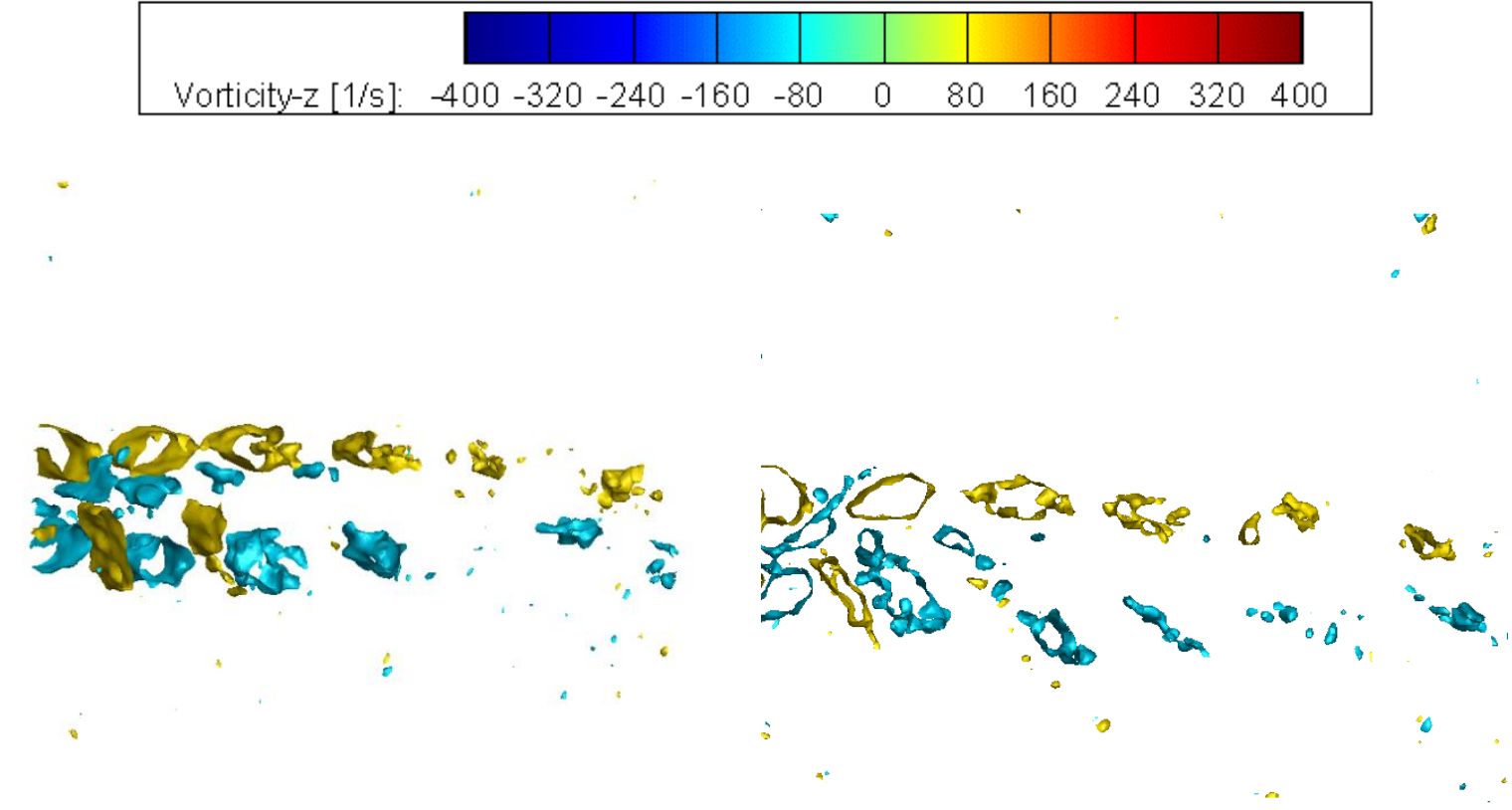

(a)

(b)

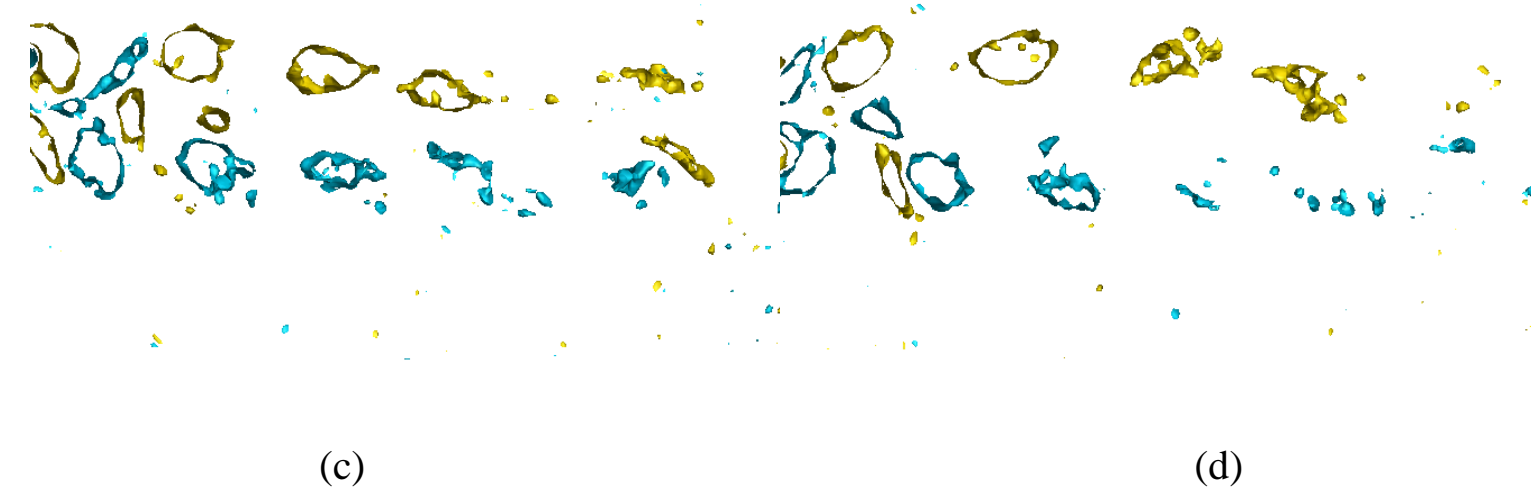

Figure 59. Iso-surface plot of $\mathrm{z}$-vorticity for $\mathrm{Re}=37$ at incident angle $0^{0}$ 

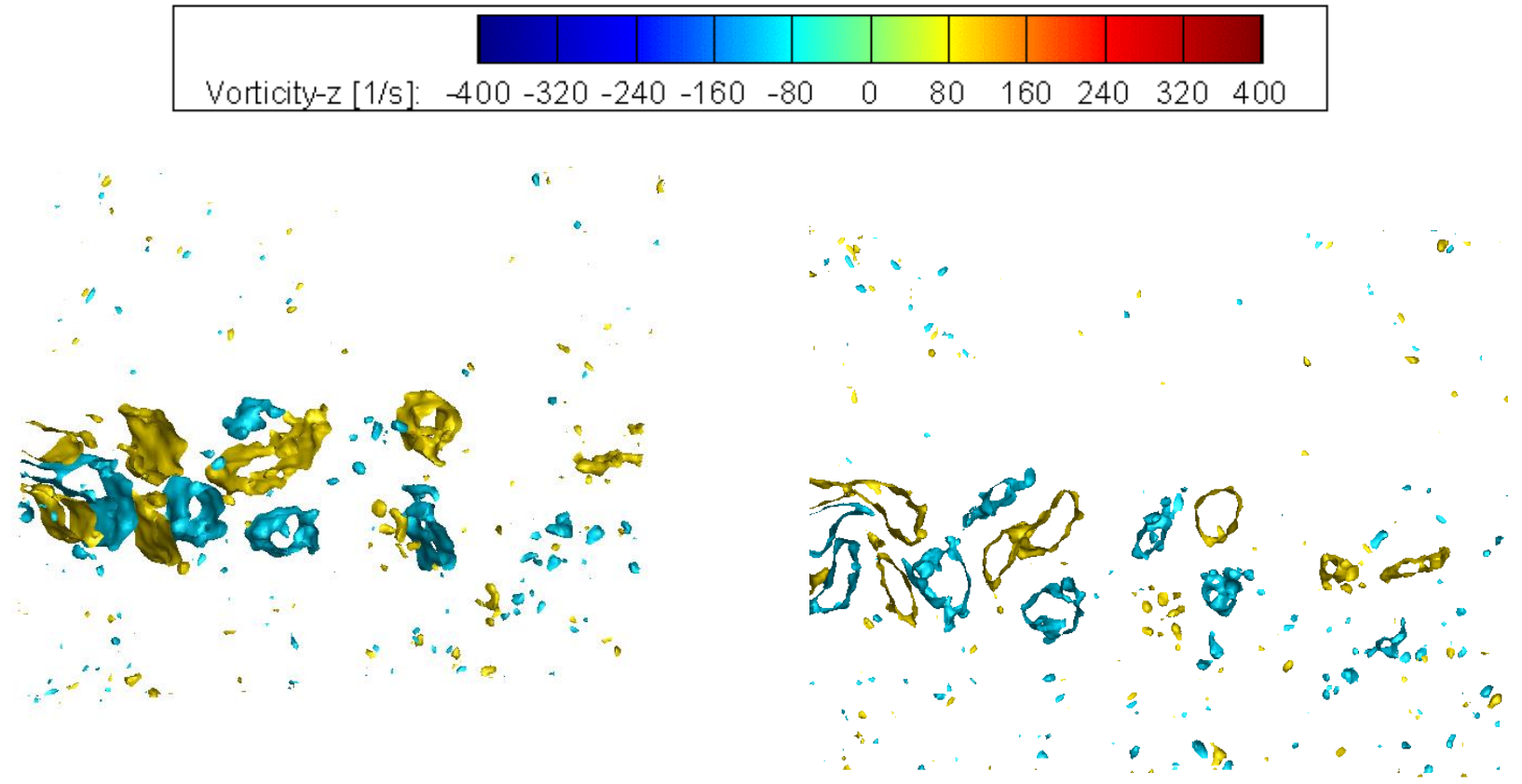

(a)

(b)

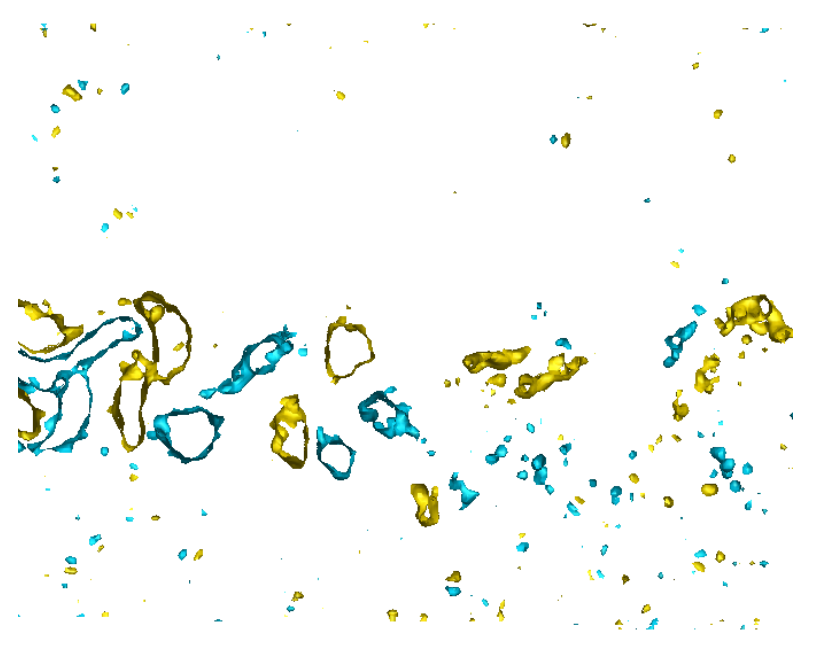

(c)

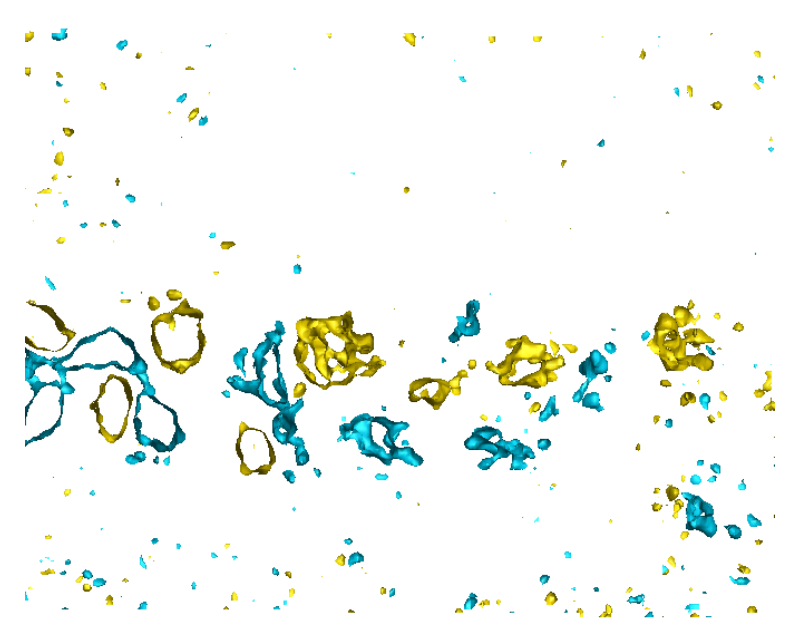

(d)

Figure 60. Iso-surface plot of $\mathrm{z}$-vorticity for $\mathrm{Re}=37$ at incident angle $45^{0}$ 


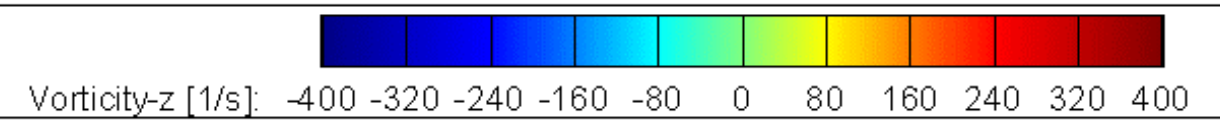

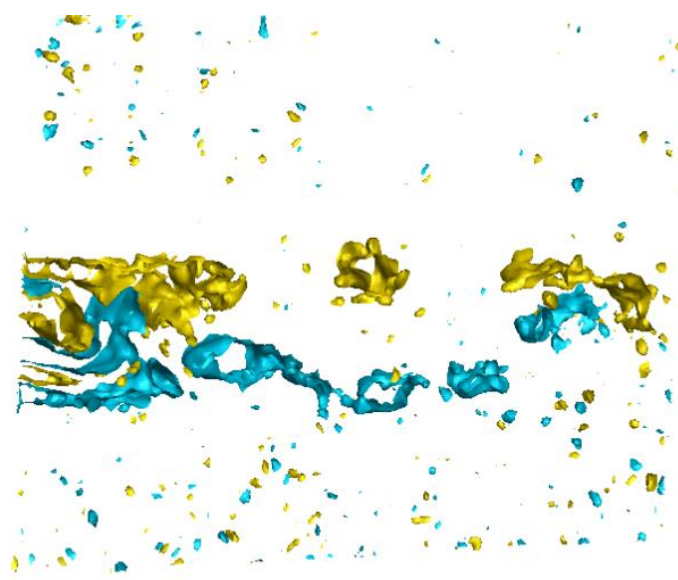

(a)

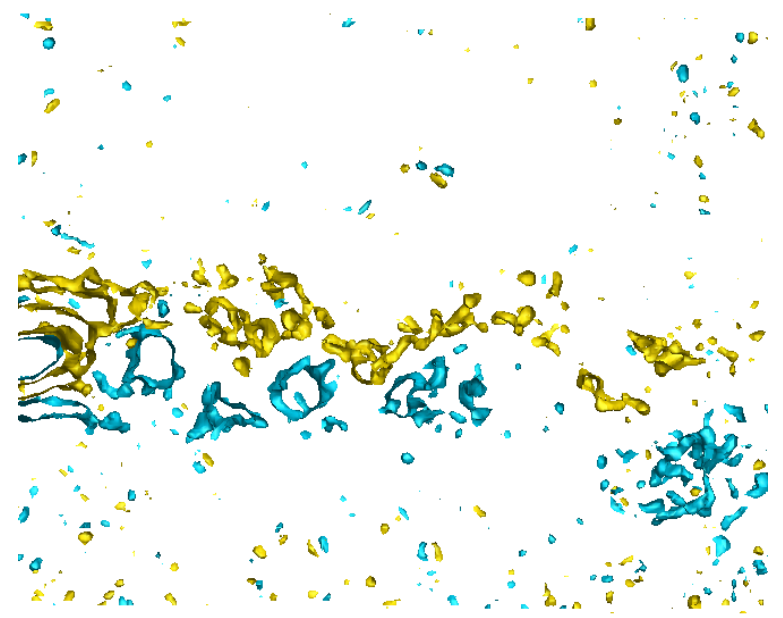

(c)
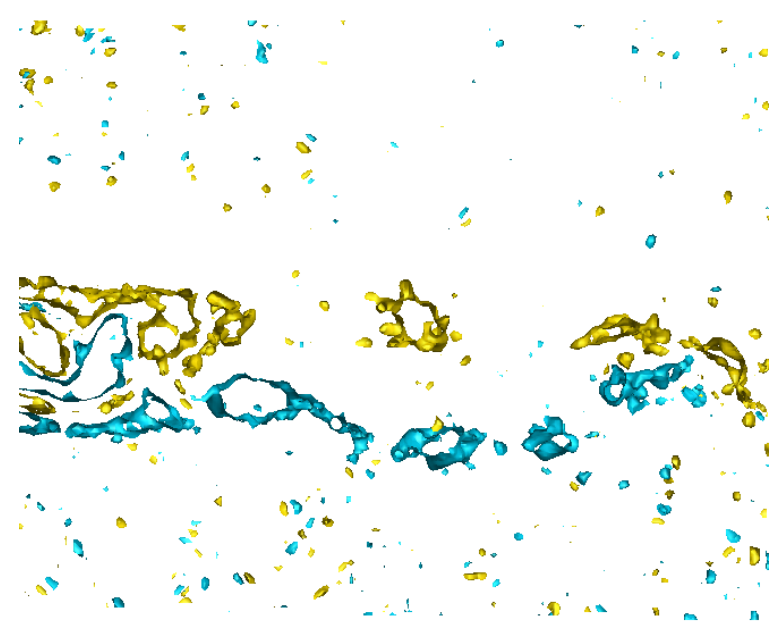

(b)
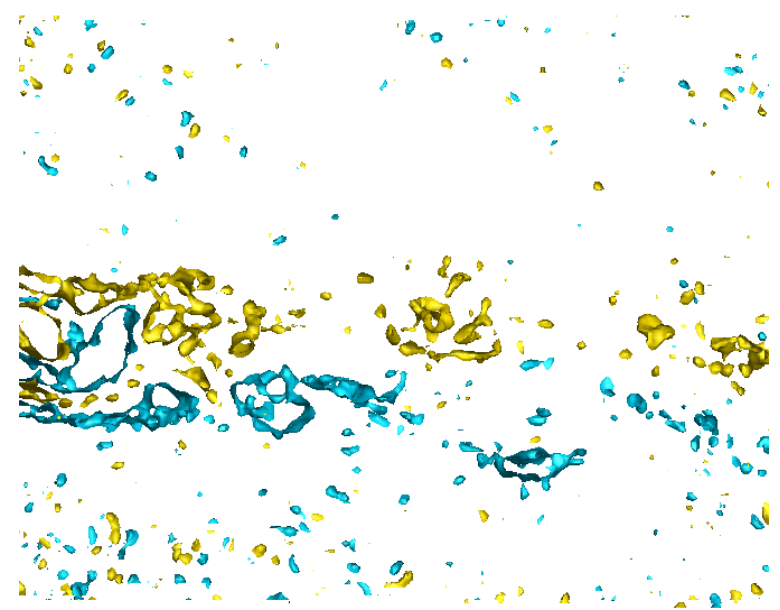

(d)

Figure 61. Iso-surface plot of z-vorticity for $\mathrm{Re}=37$ at incident angle $180^{\circ}$ 


\section{Uncertainty of Experiment}

\section{Hotwire}

Hotwire experiment has many source of error. The sources of error in hotwire experiments are following: calibration measurement errors (CME), calibration equation errors (CEE), calibration drift errors (CDE), approximation errors (AE), high frequency errors (HFE), spatial resolution error (SRE), and disturbance error (DE). So the total error associated with hotwire will be -

$$
\begin{aligned}
& \text { Uncertainty } \\
& =\sqrt{(C M E)^{2}+(C E E)^{2}+(C D E)^{2}+(A E)^{2}+(H F E)^{2}+(S R E)^{2}+(D E)^{2}}
\end{aligned}
$$

The calibration measurement errors are found from various sources. The error occurs due to analogue to digital conversion. The calibration measurement error is considered about $2 \%$. The calibration equation errors are quantified as $0.1 \%$. The calibration drift error errors are caused by variation in calibration over time and switching and this error is considered as $1.5 \%$. The other errors can be considered as negligible. The total uncertainty found in hotwire experiment is about $2.5 \%$.

\section{D Particle Image Velocimetry}

PIV is well established experimental method in fluid mechanics that has been developed over last 30 years. In our experiment we used setup from LaVision. The recent developments were able to get rid off systematic and random error source [31, 32]. However, there are other uncertainty that is still a challenge. Quantifying the remaining measurement uncertainties for all experimental and processing parameters - seeding density, out-of-plane-motion, interrogation window size, etc. - has remained a significant source of error . Since PIV evaluates the change in 
distance over a known time interval of an individual seed particle the result is a velocity vector field. That field given by the equation.

$$
U=\frac{D x}{D t} \times M
$$

Where $\mathrm{M}$ is a magnification factor determined during camera calibration. If we express the percentage error in each value of the above equation as $\delta(\mathrm{Dx}), \delta(\mathrm{Dt})$, and $\delta(\mathrm{M})$ then the overall percentage error is calculated using the Kline-McClintock measurement uncertainty formula where-

$$
\delta U=\sqrt{(\delta D x)^{2}+(\delta D t)^{2}+(\delta M)^{2}}
$$

This PIV study uses a two-dimensional image to capture the flow field where the magnification factor gives about $0.5 \%$ error. The timing unit used in this setup has an output resolution of $20 \mathrm{~ns}$ meaning an error of $0.01 \%-0.04 \%$ of actual time difference. Lastly, the image pixel resolution is estimated at around 0.1 pixel which translates to about $1.23 \%-1.73 \%$ error in the image displacement. Combining the individual errors in the Kline-McClintock uncertainty equation the total error is found to be $1.32 \%-1.80 \%$.

\section{Vorticity Wavelength Uncertainty}

In this study, the wavelength from the vorticity contour was calculated based on handpick selection. At low Re number the vorticity locations are mostly phase-locked spatially. However, at high Re number due to the unsteady nature of flow the vorticity calculation in one sample lead to significant error. This lead to a signification error in the calculation of Strouhal number. 


\section{CHAPTER 5. PROPER ORTHOGONAL DECOMPOSITION (POD) ANALYSIS}

Propoer Orthogonal Decomposition (POD) was first introduced in fluid mechanics by Lumley [24] in order to describe the flow coherent structure. Since PIV method generates large number of data, 'snapshot' based POD gained much popularity over 'classical' POD methods from the perspective of computational efficiency. Snapshot based POD was first introduced by Sirovich in 1987 [25] . After that lot of studies have used POD analysis to extract coherent structure from flow field [27-30]. In snapshot based POD method, each instantaneous velocity plot is considered as one snapshot. In this experiment we have taken 100 snapshots for POD analysis. The first step is to calculate the mean velocity from 100 of the instantaneous velocity fields. The discrete PIV data obtained from each instantenous velocity are arranged in vector $x_{i}$. The fluctuating part of all velocities in the domain of $\mathrm{i}$-th snapshot are obtained by subtracting the average from $\mathrm{x}_{\mathrm{i}}$. Therefore, with $\mathrm{n}$ snapshot vectors $\mathrm{v}_{\mathrm{i}}$ will be-

$$
v_{i}=x_{i}-\frac{1}{n} \sum_{j-1}^{n} x_{j}, i=1 \ldots \ldots . n
$$

From these vectors autocovariance matrix, $\mathrm{R}$, is formed.

$$
R_{i k}=v_{i} \cdot v_{k}
$$

Solving the algebraic eigenvalue problem for this matrix an orthonormal basis can be constructed as

$$
\emptyset^{k}=\frac{\sum_{i=1}^{n} g_{i}^{k} v_{i}}{\left\|\sum_{i=1}^{n} g_{i}^{k} v_{i}\right\|}
$$

In equation (3) $g_{i}^{k}$ is the i-th component of the normalized eigen vector correnspondig to $\mathrm{k}$-th eigenvalue, $\lambda_{k}$. Ordering the eigenvalue as $\lambda_{1}>\lambda_{2}>\cdots>\lambda_{n-1}>0$ will give the first mode, 
second mode so on. The first mode represents the most enegetic and dominant sturucture of the fluid flow and the second one contains the next dominant structure which has less energy relative to first mode.

The original flow field can be reconstructed after having the orthogonal basis from equation (4)

$$
V=\phi A
$$

Where $V, \phi$, and $A$ are metrices containing the $\phi^{k}, v_{k}$, and $a^{k}$. In order to reconstruct the snapshot based on most energetic mode, equation (4) can be used to find the coefficient $a^{k}$. Knowing the coefficient $a^{k}$, the reconstruction can be obtained by using equation (5) and (6) :

$$
\begin{gathered}
A=\phi^{T} V \\
x_{k}=\sum_{i=1}^{m} \phi^{i} a_{i}^{k}+\frac{1}{n} \sum_{j=1}^{n} x_{j}
\end{gathered}
$$

The value of $\mathrm{m}$ indicates the number of basis mode that has been considered in the reconstruction. For decomposing and reconstructing the velocity datathe data into different mode, we used DaVis software. 


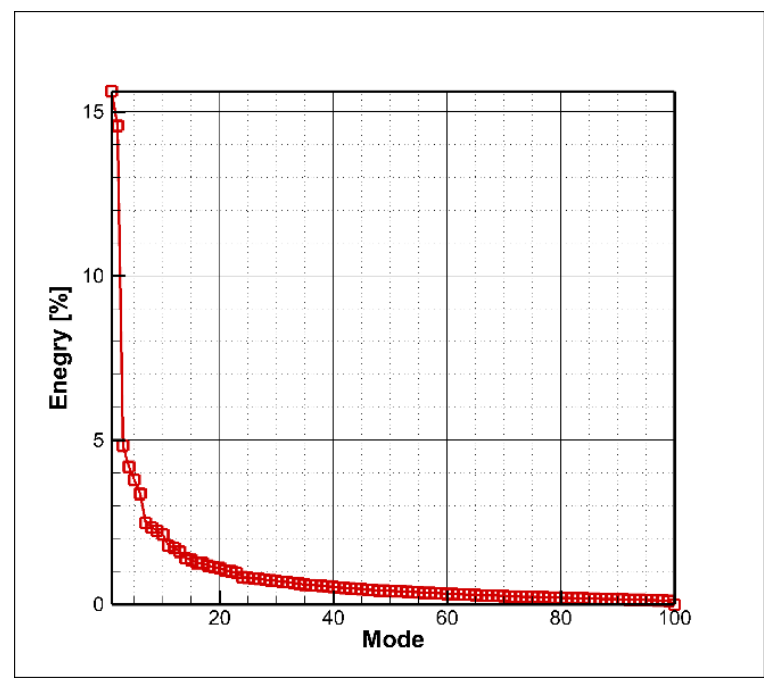

(a)

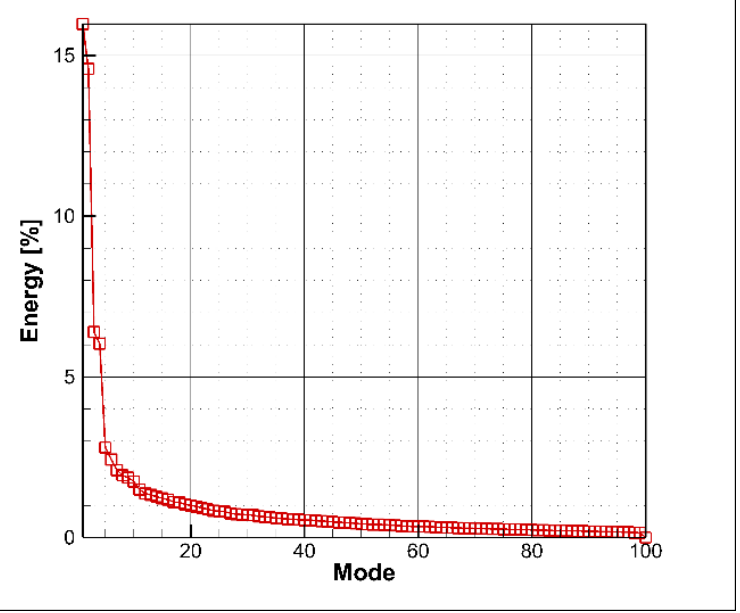

(b)

Figure 62. Relative Energy for 100 POD modes (a) Static at $0^{\circ}$ incident angle (b) Static at $180^{\circ}$ incident angle

Figure 62 shows the relative energy of 100 modes for two static cases of incident angle $0^{0}$ and $180^{\circ}$. The first 10 modes contains approximately 60 percent of total energy compare to 100 modes. The curve for both cases rapidly decreases for first 10 modes and then declines slowly after that. Since the first 10 modes carries most of the energy, it is good enough to reconstruct the velocity based on first 10 modes.

A selection of POD modes is shows in Figure 63 and Figure 64. The vectors were scaled by relative 0.3 factor. The results indicate that for first couple of mode contain detail flow structure. With increase of mode number small vortex structure appear in the contour. Considering the first 10 modes, mode 80 and 90 represent small scattered vortices over the domain which contain very small amount of energy. This is in agreement with the theoretical description of the POD modes and also proves the importance of decomposing data into an orthogonal basis for deeper level of analysis. From the energy plot in Figure 62, we can reconstruct the velocity based on first 10 modes as they contain most of the energy. By using Equation 06, the reconstructed velocity for both condition is shown in Figure 65 and Figure 66. First six reconstructed velocity based on first 10 
modes is plotted for both cases. The result fundamental expected wake phenomena for $0^{\circ}$ and $180^{\circ}$. The dominate mode indicate that the existence of downstream cylinder at intermediate spacing dominates the flow. The wake from the upstream cylinder is not strong enough to travel in the downstream. This characteristics is caught by the reconstructed velocity contour.

In Figure 67 and Figure 68, the instantaneous velocity contour and reconstructed velocity contour based POD analysis is shown side by side for first three instances. From the comparison we can see that the reconstructed velocity based on first 10 modes represent the real velocity contour that is obtained from PIV data. Reconstructed velocity offers more clean data by reducing noise level. 

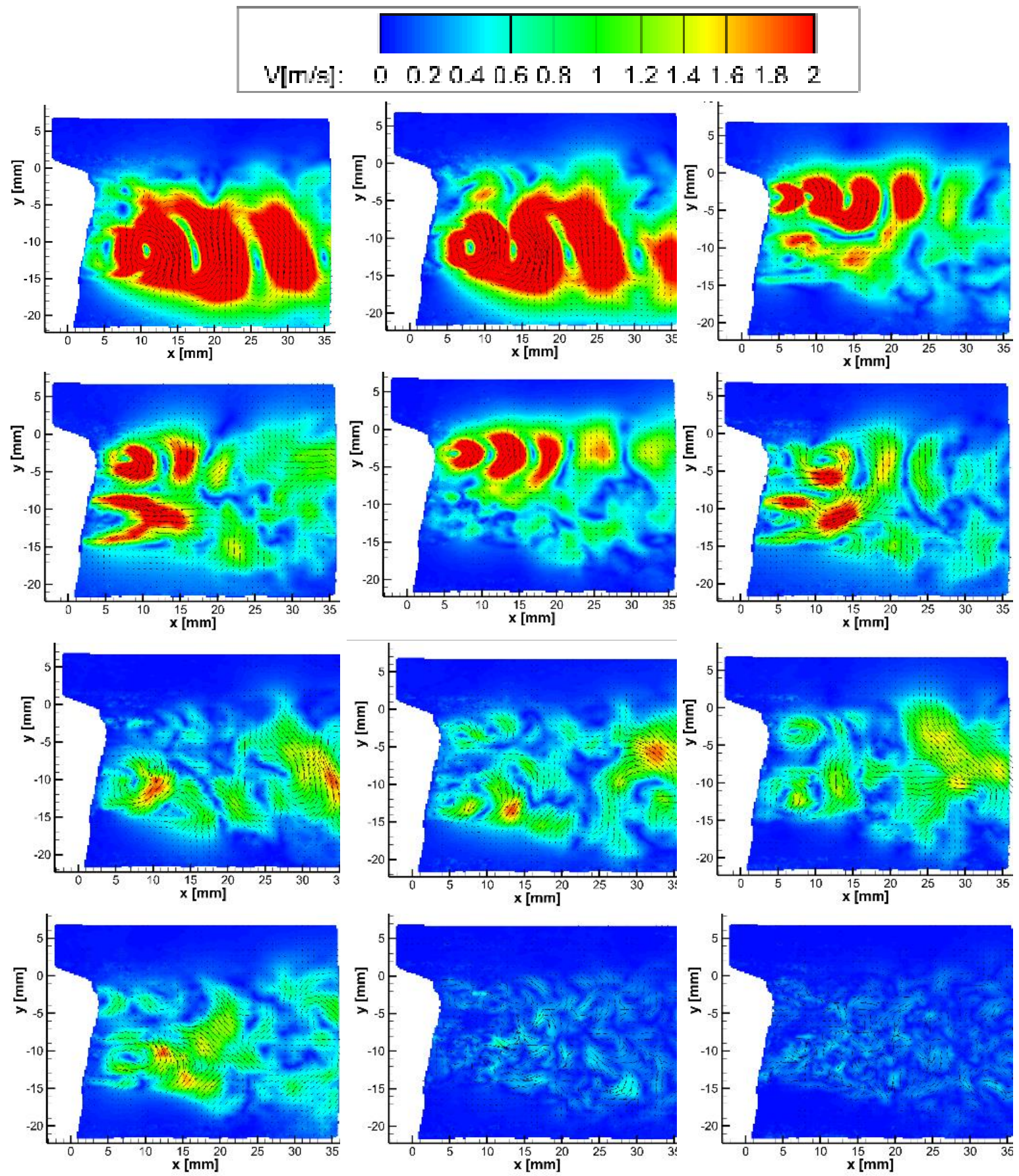

Figure 63. POD modes 1-10,80, 90 (from left to right) for Static $0^{0}$ Incident angle at $\mathrm{Re}=57$ 

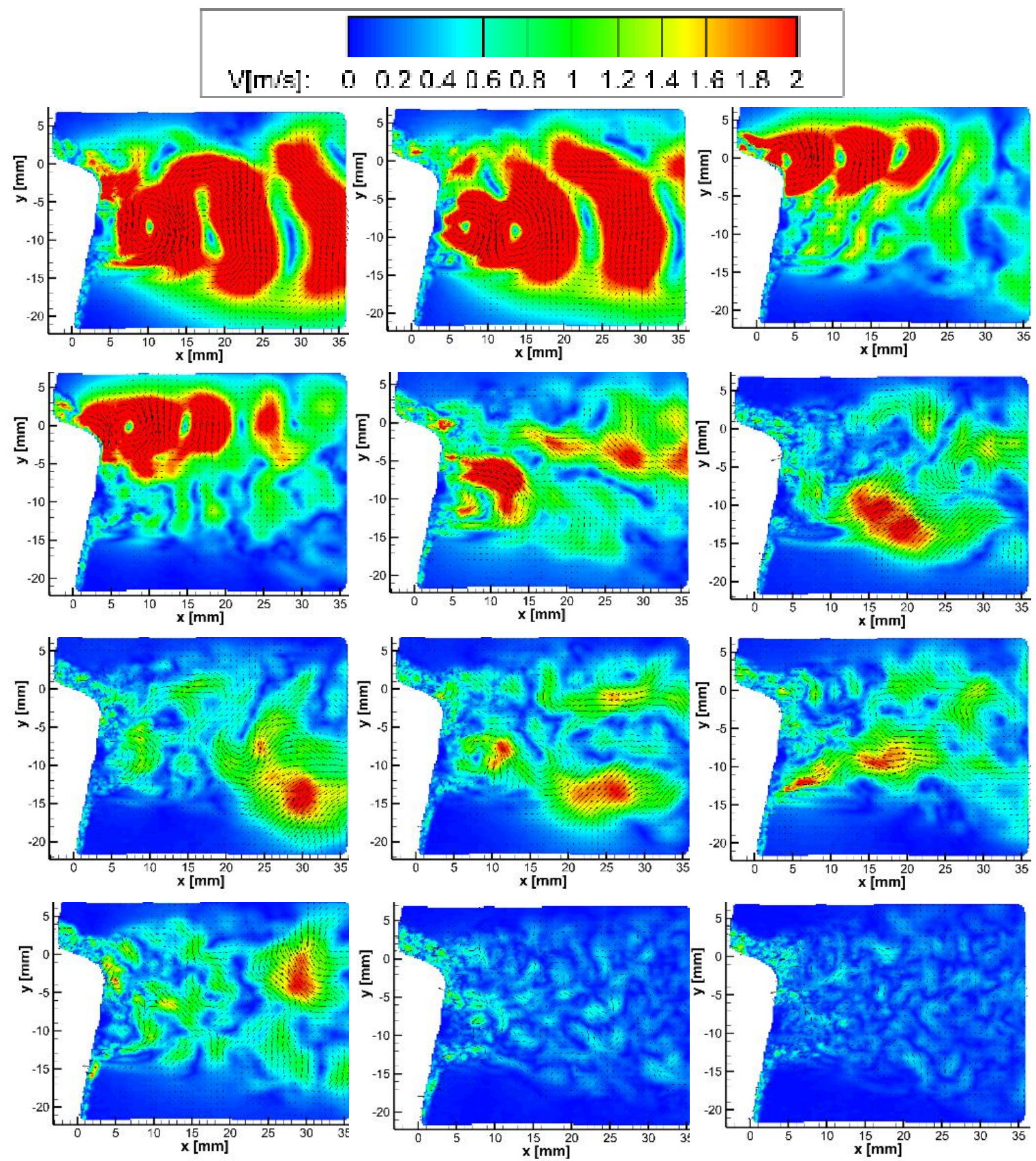

Figure 64. POD modes 1-10,80, 90 (from left to right) for Static $180^{\circ}$ Incident angle at $\operatorname{Re}=57$ 

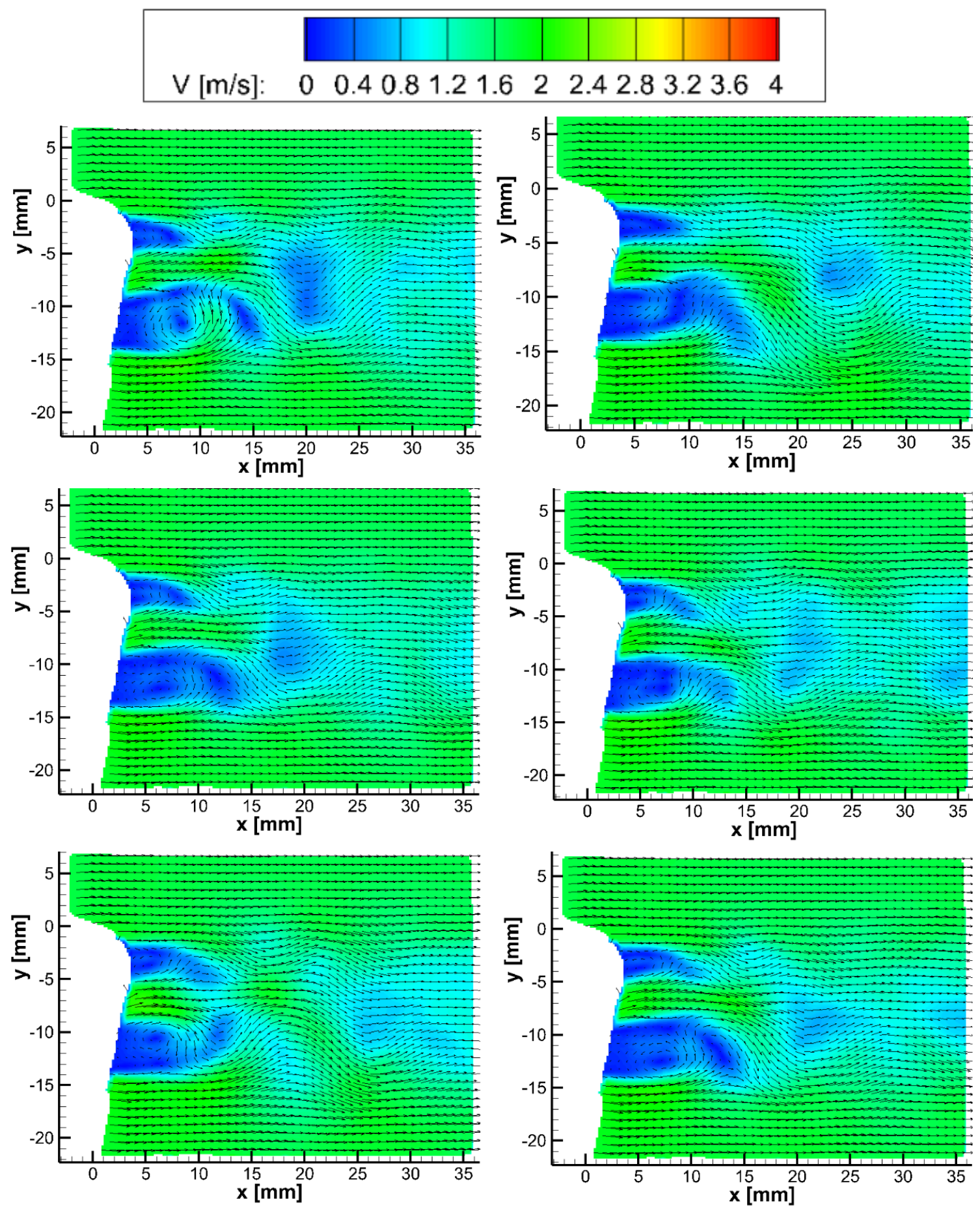

Figure 65. First six (left to right) reconstructed velocity contour based on 10 POD modes for Static $0^{0}$ incident Angle at $\mathrm{Re}=57$ 

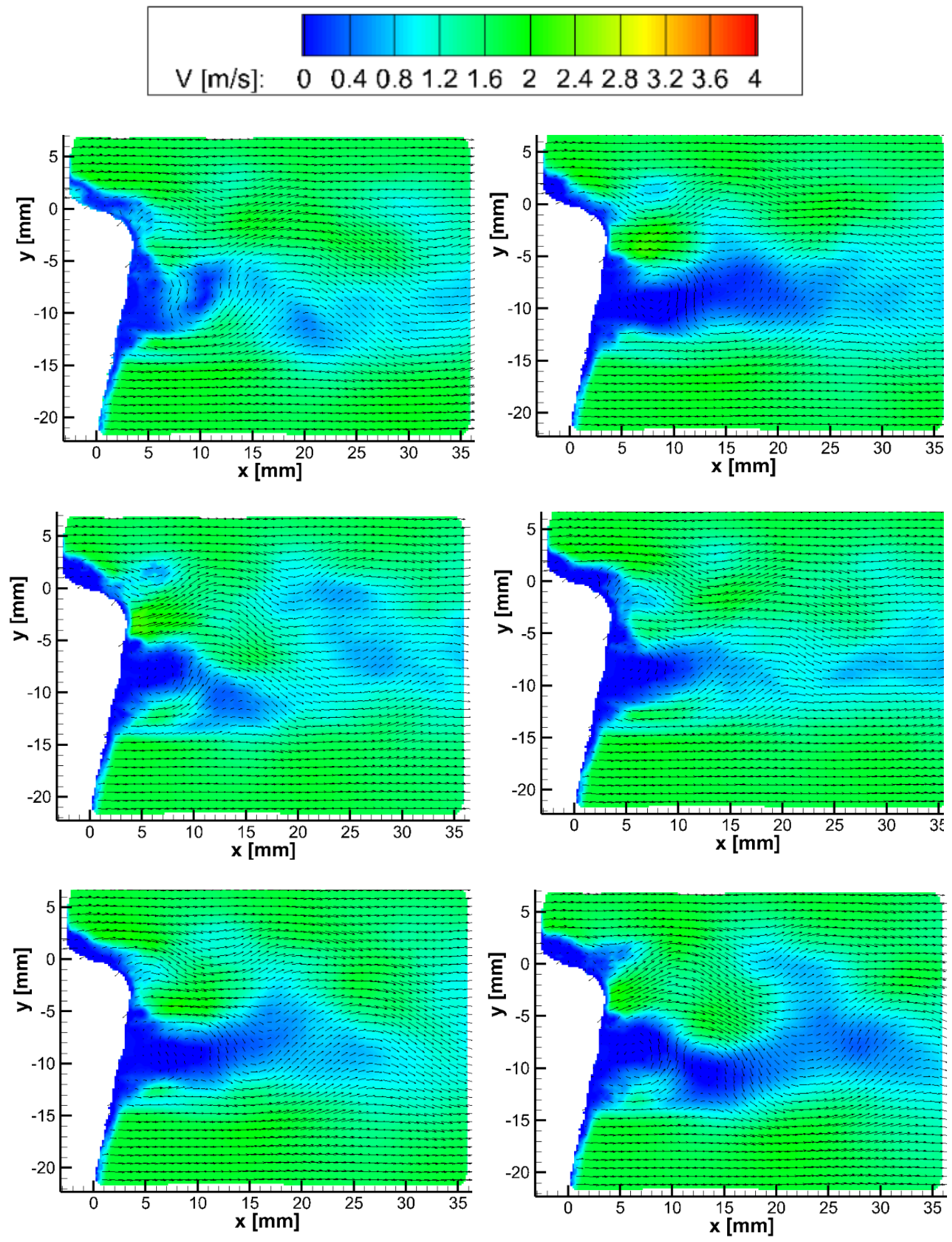

Figure 66. First six (left to right) reconstructed velocity contour based on 10 POD modes for Static $180^{\circ}$ incident Angle at $\mathrm{Re}=57$ 

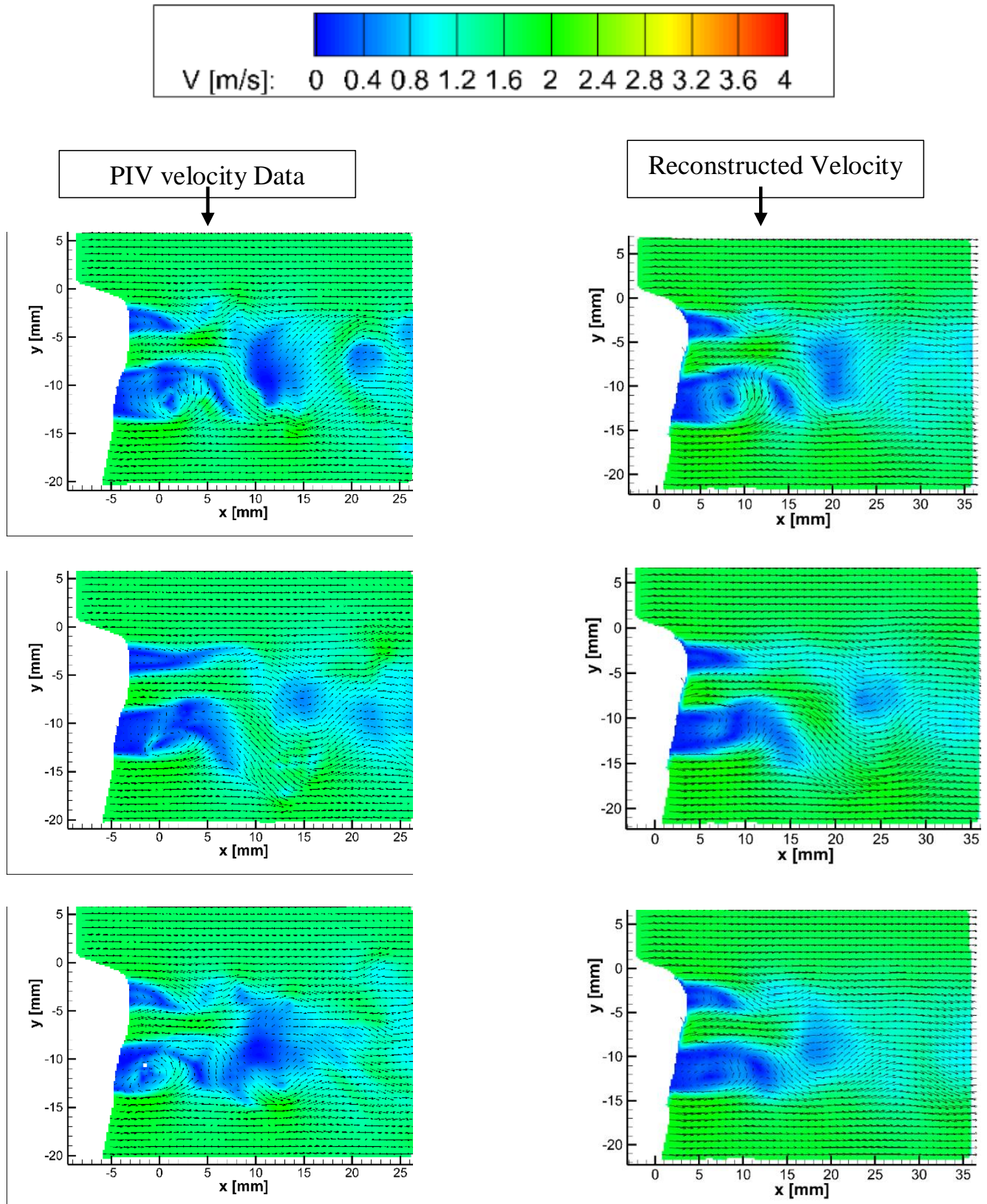

(a)

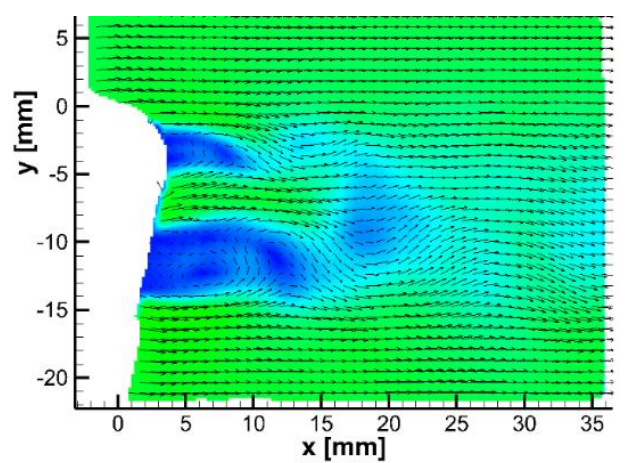

(b)

Figure 67. Comparison of first three PIV instantaneous velcoity contour (a) with Reconstructed velocity contour based on POD analysis for $0^{0}$ incident angle at $\mathrm{Re}=57$ 

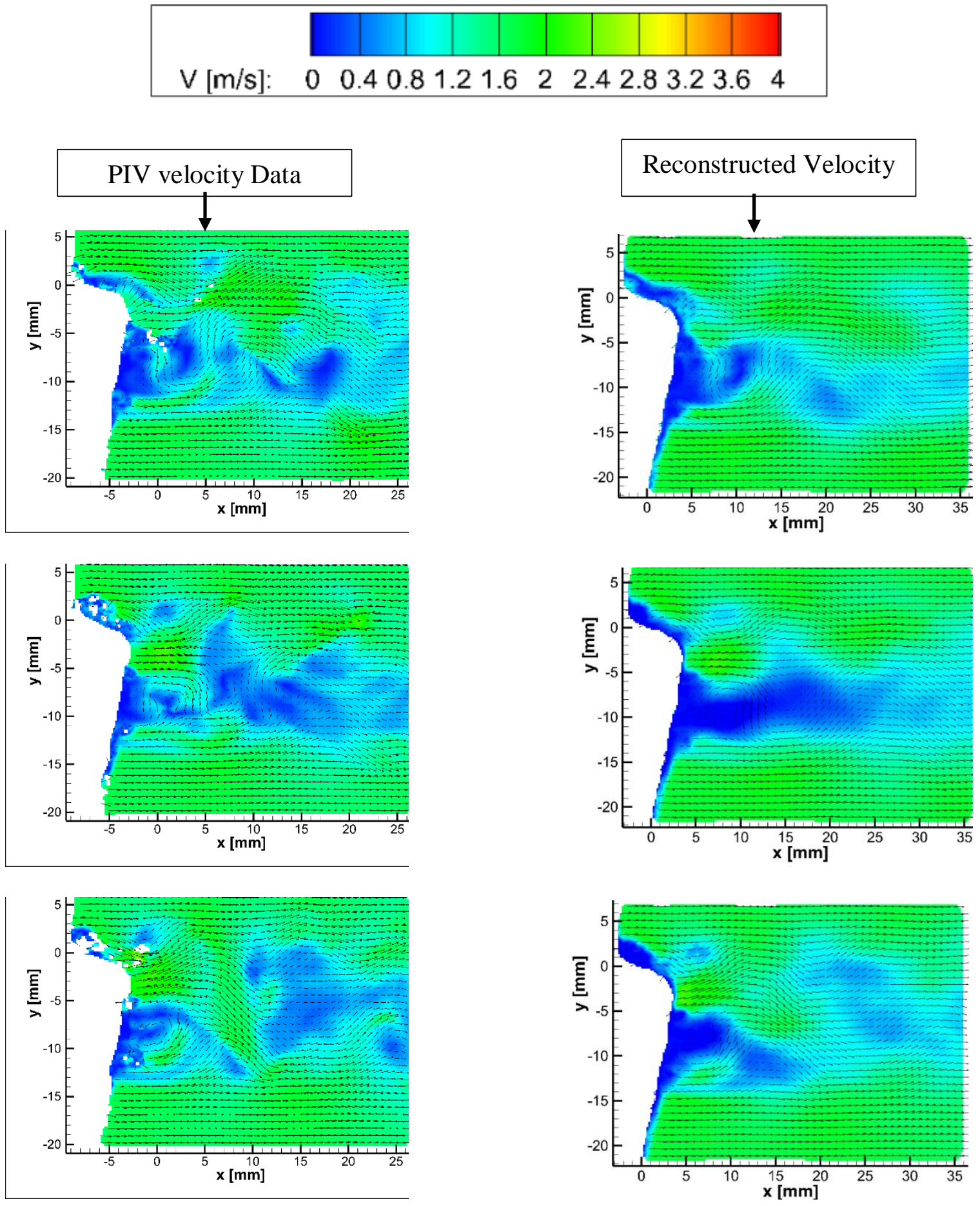

(a)

(b)

Figure 68. Comparison of first three PIV instantaneous velcoity contour (a) with Reconstructed velocity contour based on POD analysis for $180^{\circ}$ incident angle at $\mathrm{Re}=57$ 


\section{CHAPTER 6. SUMMARY}

In this chapter, the summary of the findings from the Hotwire, PIV, and tomography experiments is discussed. With the hotwire experiment, the Strouhal number was captured from both static and rotating conditions. Note that the diameter of the single-cylinder was taken as characteristic length to calculate the Strouhal number. For static conditions, the St value was captured mostly with $45^{\circ}$ and $180^{\circ}$ orientation. The St value is shown as a function of Re number in Figure 11. Figure 11 shows that a sudden increase and decrease in St value and the magnitude of St value is higher than single-cylinder vortex shedding for some instances. This pattern was also found in the study done by Lam, K. et al. [1]. This phenomenon happens because of the intermediate spacing to the diameter ratio of the cylinder arrangement. At intermediate spacing, wake from the inner side of upstream cylinders is deflected due to the presence of a cylinder at downstream. The deflection of the wake is thought to be the reason for the high frequency of vortex shedding. The wake deformation of the individual cylinder is captured in our PIV study (Figure 13). Based on the findings from both PIV campaign, a schematic of wake interaction from individual cylinder is shown in Figure 69. Wake interaction exhibits different pattern of interaction with the change of interaction. When the cylinder arrangement is $0^{0}$, the wake from upstream cylinder reattaches with innder side of the downstream cylinder wake. The counter rotating spin of two adjacent vortices weakens the wake from upstream cylinder. As a result two wakes travels downstream and evolves to ring vortices. At $45^{\circ}$ static case, the wake from upstream cylinder reattaches with the downstream cylinder's wake and the wake from the lower downstream cylinder combines with upper cylinder. The combination creates an asymmetric vortex shedding. As we change the cylinder orientation to $180^{\circ}$, the wake from upstream cylinder deflects and narrowed due to the presence of downstream cylinder. A symmetric wake is observed from the downstream 
cylinder and a synchronized vortex shedding happens at this particular orientation. These phenomenon at static cases are found in our both campaign of PIV experiment. Also, these findings are also very well known pattern and in agreement with the previous computational study done by Bao, Y. et al. [2]. For static case our findings in PIV study is consistent with tomography data. The tomography data also shows similar well know wake patter with three dimensional iso-surface vortex plot which offers more detail of flow.

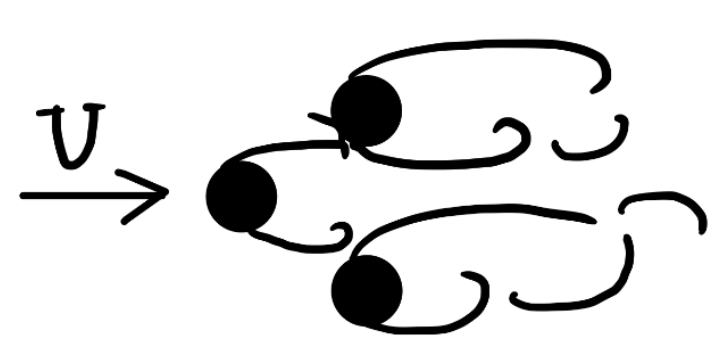

(a)

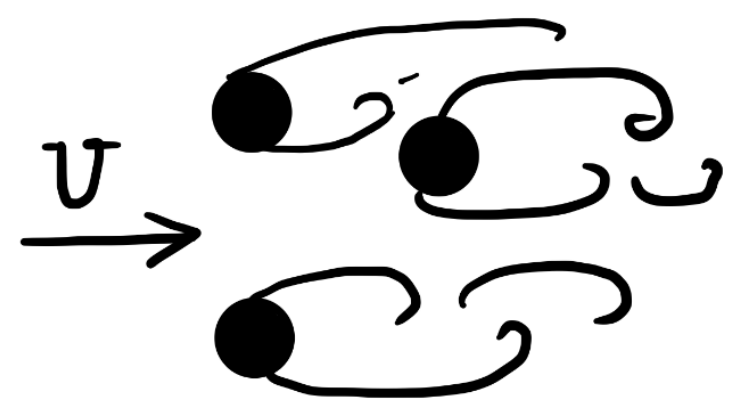

(b)

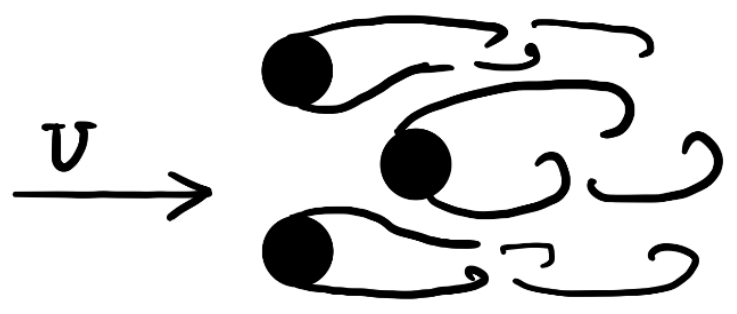

(c)

Figure 69. Schematics of flow structrue for static cases (a) $0^{\circ}$, (b) $45^{\circ}$, and (c) $180^{\circ}$

Inclusion of the rotation of cylinder system in this study shows unique results and new wake characteristic. From the hotwire experiment, the results show distinct peaks in the frequency domain which is converted from the time signal captured by hotwire. After converting all the time signal to frequency domain, the St value was plotted as a function of RPM and Re number. For rotating conditions, the diameter of the circle escribed to the equilateral arrangement of cylinder system was considered as characteristic length. From the plot of St vs RPM (Figure 12), the results 
indicate that St number is high at low $\mathrm{Re}=57$ compare to other Re number. When the hotwire position changes longitudinally, the pattern of the St Plot changes with RPM for specific Re number. This pattern is observed from the comparison of Figure 12 (b-d). In addition, the results also show sudden increase and decrease of St Value with RPM for mid-range Re number, for example $\operatorname{Re}=500,858$ and 1250. As the Re number increases very high, for example $\operatorname{Re}=4350$, and 7470, the St Curve follows almost linear pattern with RPM. The hotwire data indicates the vortex shedding is unique with rotation of cylinder. This was further proved by our PIV study. In the first Step, the panoramic view of vorticity plot (obtained from two camera PIV that was set to see far view) at low Re number shows the rotation of cylinder breaks the wake into smaller positive and negative vortices and their mixing causes laminar to turbulent transition. The counter clock wise rotation of cylinder enhances vortex shedding and mixing in the top side of the flow field which increases the relative velocity at highest RPM. The counter interaction in the bottom diminishes and cancels vortex shedding at highest RPMAs the Re number increases the vorticity becomes stronger and strong mixing of positive and negative vortices can be observed in Figure 28. The standard deviation shown in Figure 18 to Figure 22 proves the high turbulent intensity with higher RPM and Re number due to the mixing of positive and negative vortices. Similar trend of results is also obtained in the second phase of PIV study, which also offer detail flow structure obtained by close view of two camera. The schematics figure is shown based on our finding in PIV campaign in Figure 70. The schematics diagram was drawn after observing few instances. The continuous rotation of cylinder makes the separation of the flow earlier compare to static case and sheds as small vorticies. The positive and negative vortices in wake region continuously mixes and generates high turbulence in the flow domain. Later the average velocity profile in show how the velocity profile changes in different downstream position. The average velocity was calculated 
based on 100 instantaneous time uncorrelated velocity contour. Significant velocity change is observed in the upstream for various RPM compare to downstream location. As the Re number increases the velocity profile start to become similar at downstream location.

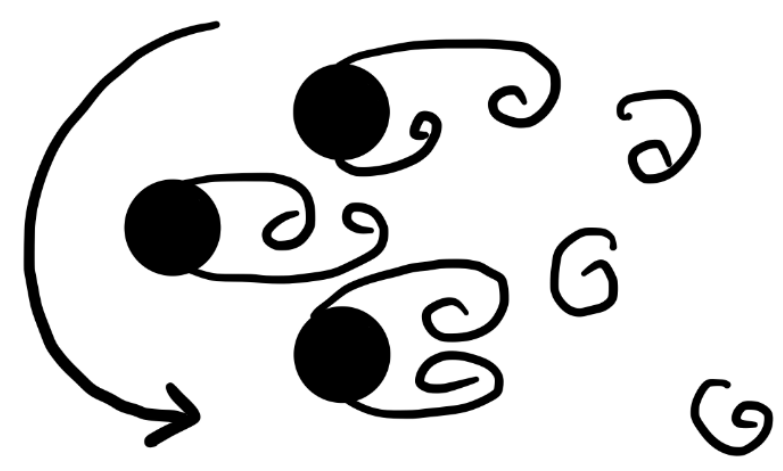

Figure 70. Schematics of flow structrue for rotating conditions

In last section, POD analysis was performed to show the decomposition of velocity contour and the reconstruction based on dominant mode. The comparison of reconstructed velocity and PIV velocity is shown in Figure 67 and Figure 68 for $0^{\circ}$ and $180^{\circ}$ static phase angle. The results shows that the reconstructed velocity based on first 10 modes represents the actual velocity obtained from PIV. This analysis helps as computational basis to model the flow field. 


\section{CHAPTER 7. CONCLUSION}

Flow past three cylinder measurement was done experimentally using hotwire, 2D PIV and tomography study. An equilateral triangular arrangement of cylinder with intermediate spacing was used to do the measurements. For non-rotating cases, our findings with hotwire matches with the previous study. In addition, 2D PIV reveal the well know wake structure for specific orientation that was also found in previous experimental and computational study. Later, the results from tomography study is consistent with our PIV study and offers 3D structure of flow field.

Furthermore, the rotating cases in our study add new dimension and reveal interesting characteristics of flow. The hotwire relation gives us time resolved data which further provides the relation of non-dimensional Strouhal number as a function of RPM and Re. The anomaly of vortex shedding frequency of rotating cases with single cylinder was further proved by flow visualization and flow field visualization. This reveal peculiar characteristics which thought to be the reason of having different vortex shedding than a single cylinder vortex shedding. The plot of vorticity, standard deviation and wake profile proves the flow is highly unsteady in nature and shows reason for laminar to turbulent transition. Further investigation is necessary with different optical setup to get the flow structure across the span of cylinder. Also more hotwire measurement will help to find how the vortex shedding frequency varies with longitudinal and transverse position. However, this study will have sufficient data to provide basis for computational modeling and validation. NDSU CFD group is currently running a simulation of various cases in order to describe the flow characteristics and the findings from this study is going to basis for comparison. The experimental findings are going to be considered as basis in order to validate the model of CFD data. 


\section{Future Work}

Advance diagnostics technique has been applied to characterize the flow past threecylinder system having intermediate spacing ration. This work can be further explored for various spacing ratio. In addition, Hotwire experiment can be done further at different position based on PIV results so that we can capture the vortex shedding frequency for most of the static cases. Another comprehensive study can be done in order to publish the work in peer review journal is to get hotwire data at small distance interval. This will help us to understand how the vortex shedding frequency changes with the change of $\mathrm{x}$ and $\mathrm{y}$ position.

In order to capture the flow structure across the span of the cylinder, Laser illumination from the side wall can be done and capturing the particle movement from top in going to be another important part that needs to be conducted in order to have more 3D information of flow.

After validating these work with the simulation obtained by NDSU CFD group will be published in a peer reviewed journal. 


\section{REFERENCES}

${ }^{1}$ Lam, K., and Cheung, W., 1988, "Phenomena of vortex shedding and flow interference of three cylinders in different equilateral arrangements," Journal of fluid mechanics, 196, pp. 1-26.

2 Tatsuno, M., Amamoto, H., and Ishi-i, K., 1998, "Effects of interference among three equidistantly arranged cylinders in a uniform flow," Fluid dynamics research, 22(5), p. 297.

${ }^{3}$ Bao, Y., Zhou, D., and Huang, C., 2010, "Numerical simulation of flow over three circular cylinders in equilateral arrangements at low Reynolds number by a second-order characteristicbased split finite element method," Computers \& Fluids, 39(5), pp. 882-899.

${ }^{4}$ Zdravkovich, M., 1968, "Smoke observations of the wake of a group of three cylinders at low Reynolds number," Journal of Fluid Mechanics, 32(2), pp. 339-351.

${ }^{5}$ Ullah, A. H., Rostad, B., and Estevadeordal, J., "PIV Study of the Flow Interaction between a Three-Cylinder Rotating System and a downstream Dimpled Airfoil," Proc. AIAA Aviation 2019 Forum, p. 3297.

${ }^{6}$ Prandtl, L., 1925, "The Magnus effect and windpowered ships," Naturwissenschaften, 13(6), pp. 93-108.

7 Jaminet, J., 1969, "Experiments on vortex shedding from rotating circular cylinders," AIAA Journal, 7(9), pp. 1817-1819.

8 Taneda, S., 1977, "Visual study of unsteady separated flows around bodies," Progress in Aerospace Sciences, 17, pp. 287-348.

${ }^{9}$ Diaz, F., Gavaldà, J., Kawall, J., Keffer, J., and Giralt, F., 1983, "Vortex shedding from a spinning cylinder," The Physics of fluids, 26(12), pp. 3454-3460.

${ }^{10}$ Diaz, F., Gavalda, J., Giralt, F., Kawall, J., and Keffer, J., 1985, "Asymmetrical wake generated by a spinning cylinder," AIAA journal, 23(1), pp. 49-54. 
${ }^{11}$ Coutanceau, M., and Menard, C., 1985, "Influence of rotation on the near-wake development behind an impulsively started circular cylinder," Journal of Fluid Mechanics, 158, pp. 399-446.

12 Hoffmann, J. A., 1991, "Effects of freestream turbulence on the performance characteristics of an airfoil," AIAA journal, 29(9), pp. 1353-1354.

${ }^{13}$ Kang, S., Choi, H., and Lee, S., 1999, "Laminar flow past a rotating circular cylinder," Physics of Fluids, 11(11), pp. 3312-3321.

${ }^{14}$ Chew, Y., Cheng, M., and Luo, S., 1995, "A numerical study of flow past a rotating circular cylinder using a hybrid vortex scheme," Journal of fluid mechanics, 299, pp. 35-71.

${ }^{15}$ Rao, A., Radi, A., Leontini, J. S., Thompson, M. C., Sheridan, J., and Hourigan, K., 2015, "A review of rotating cylinder wake transitions," Journal of Fluids and Structures, 53, pp. 2-14.

${ }^{16}$ Hori, E.-i., "Experiments on flow around a pair of parallel circular cylinders," Proc. Proc. 9th Japan National Congress for Applied Mech., pp. 231-234.

17 King, R., and Johns, D., 1976, "Wake interaction experiments with two flexible circular cylinders in flowing water," Journal of Sound and Vibration, 45(2), pp. 259-283.

18 Bearman, P., and Wadcock, A., 1973, "The interaction between a pair of circular cylinders normal to a stream," Journal of Fluid Mechanics, 61(3), pp. 499-511.

${ }^{19}$ Ishigai, S., Nishikawa, E., Nishimura, K., and Cho, K., 1972, "Experimental study on structure of gas flow in tube banks with tube axes normal to flow: Part 1, Karman vortex flow from two tubes at various spacings," Bulletin of JSME, 15(86), pp. 949-956.

${ }^{20}$ Tatsuno, M., Amamoto, H., Ishi-i, K., and Takayama, T., 1991, "On the stable posture of a pair of parallel cylinders in a uniform flow," Fluid dynamics research, 8(5-6), p. 253.

${ }^{21}$ Igarashi, T., and Suzuki, K., 1984, "Characteristics of the flow around three circular cylinders," Bulletin of JSME, 27(233), pp. 2397-2404. 
22 Price, S., and Paidoussis, M., 1984, "The aerodynamic forces acting on groups of two and three circular cylinders when subject to a cross-flow," Journal of Wind Engineering and Industrial Aerodynamics, 17(3), pp. 329-347.

${ }^{23}$ Kim, T., Lu, T., and Song, S. J., 2017, Application of Thermo-Fluidic Measurement Techniques: An Introduction, Butterworth-Heinemann.

${ }^{24}$ Lumley, J.L., " Stochastic tools in turbulence”. Academic Press, London, Year 1970.

${ }^{25}$ Sirovich, L, “Turbulence and the dynamics of coherent structures. Part-I: Coherent structures". Applied math 45:561-571, Octobar, 1987.

${ }^{26}$ Pedersen, J.M., Meyer, K.E., "POD analysis of flow structure in a scale model of a ventilated room”. Experiment in Fluids 33(2002), 940-949.

${ }^{27}$ Meyer, K.E., Cavar, D., Pedersen, J.M, "POD as tool for comparison of PIV and LES data". $7^{\text {th }}$ International symposium on Particle Image Velocimetry, Rome, Italy, September 11-14, 2007.

28 Druault, P., Guibert, P., Alizon, F., "Use of proper orthogonal decomposition for time interpolation from PIV data" Experiment in Fluids(2005) 39: 1009-1023

${ }^{29}$ Semeraro, O., Bellani, G., Lundell, F., “Analysis of time-resolved PIV measurements of a confined turbulent jet using POD and Koopman modes”. Exp Fluids (2012) 53:1203-1220.

${ }^{30}$ Druault, P., Chaillou, C., 'Use of Proper Orthogonal Decomposition for reconstructing the 3D in-cylinder mean-flow field from PIV data”. C. R. Mecanique 335 (2007) 42-47.

${ }^{31}$ Raffel, M., Willert, C. E., Scarano, F., Kähler, C. J., Wereley, S. T., \& Kompenhans, J. (2018). Particle image velocimetry: a practical guide. Springer.

32 Elsinga, G. E., Scarano, F., Wieneke, B., \& van Oudheusden, B. W. (2006). Tomographic particle image velocimetry. Experiments in fluids, 41(6), 933-947. 


\section{APPENDIX A}

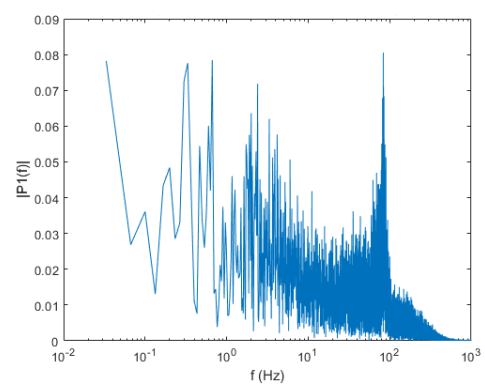

(a) $-(x / d=1.61, y / d=0)$

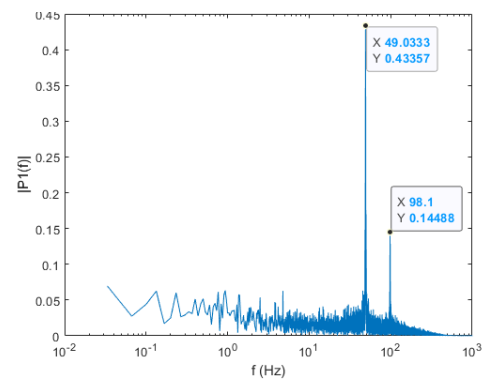

(b) $-(\mathrm{x} / \mathrm{d}=1.61, \mathrm{y} / \mathrm{d}=0)$

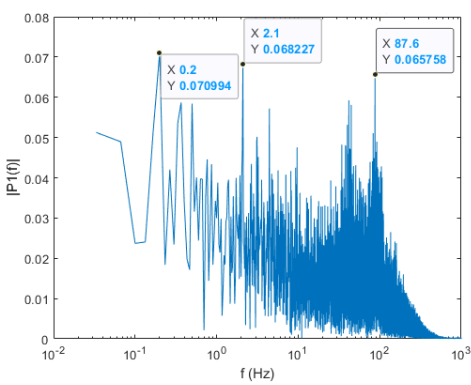

(c) $-(\mathrm{x} / \mathrm{d}=1.61, \mathrm{y} / \mathrm{d}=0)$

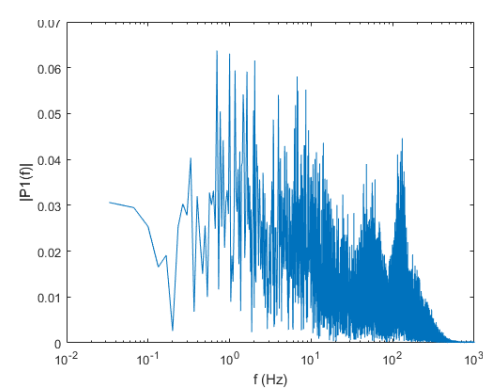

(a) $-(x / d=1.61, y / d=2.21)$

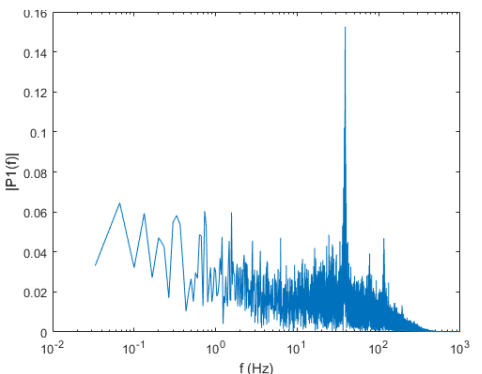

(b) $-(x / d=1.61, y / d=2.21)$

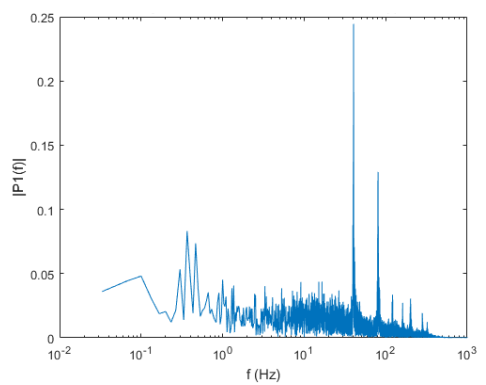

(c) $-(x / d=1.61, y / d=2.21)$

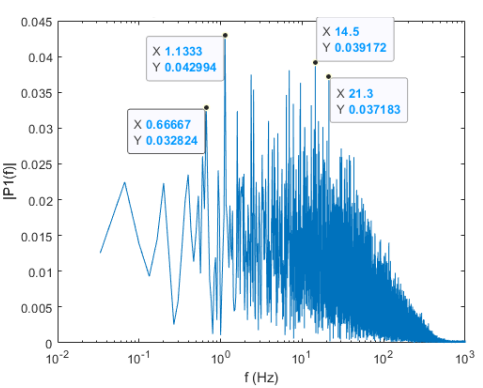

(a) $-(x / d=32.2, y / d=0)$

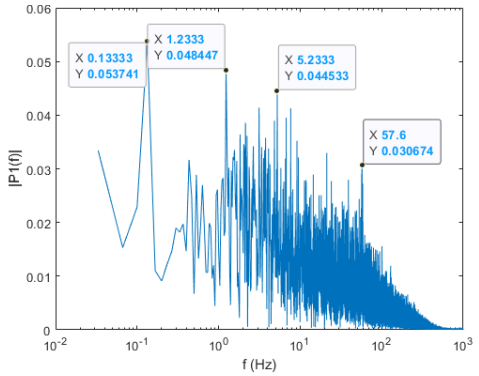

(b) $-(\mathrm{x} / \mathrm{d}=32.2, \mathrm{y} / \mathrm{d}=0)$

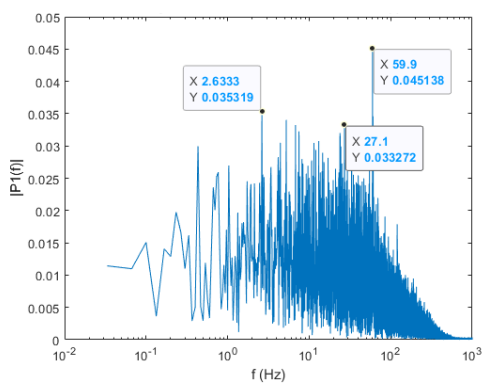

(c) $-(\mathrm{x} / \mathrm{d}=32.2, \mathrm{y} / \mathrm{d}=0)$

Figure A.1. Single sided frequency spectrum at Reynold Number 57 and incident angle (a) $0^{0}$ (b) $45^{\circ}$ and (c) $180^{\circ}$ 


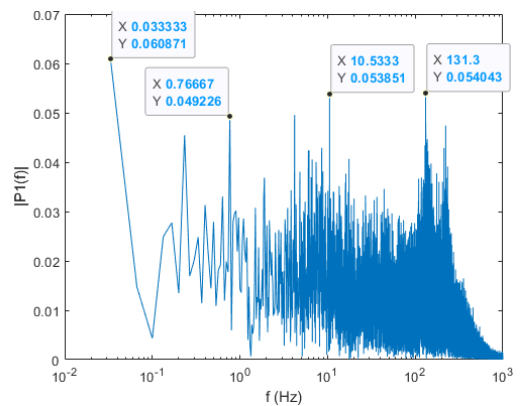

(a) $-(\mathrm{x} / \mathrm{d}=1.61, \mathrm{y} / \mathrm{d}=0)$

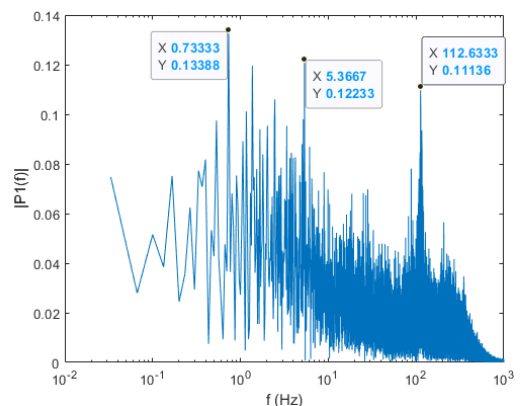

(b) $-(x / d=1.61, y / d=0)$

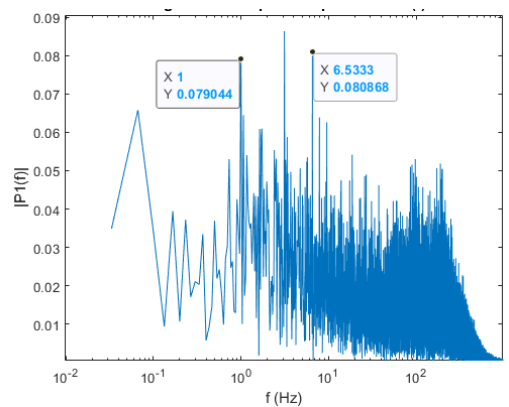

(c) $-(\mathrm{x} / \mathrm{d}=1.61, \mathrm{y} / \mathrm{d}=0)$

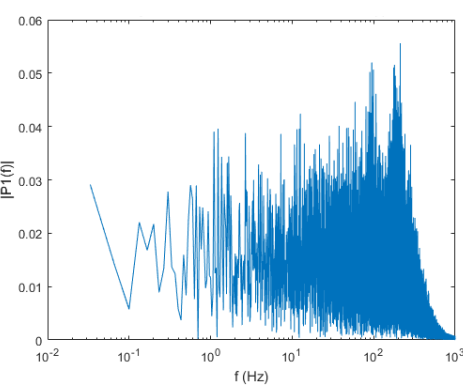

(a) $-(x / d=1.61, y / d=2.21)$

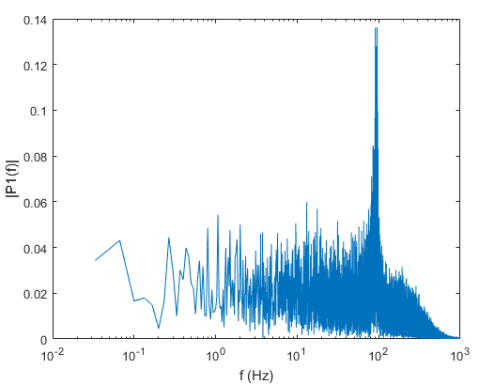

(b) $-(x / d=1.61, y / d=2.21)$

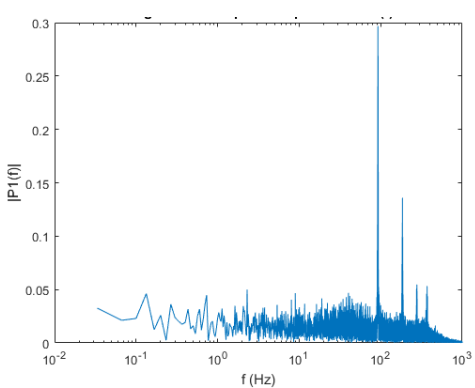

(c) $-(\mathrm{x} / \mathrm{d}=1.61, \mathrm{y} / \mathrm{d}=2.21)$

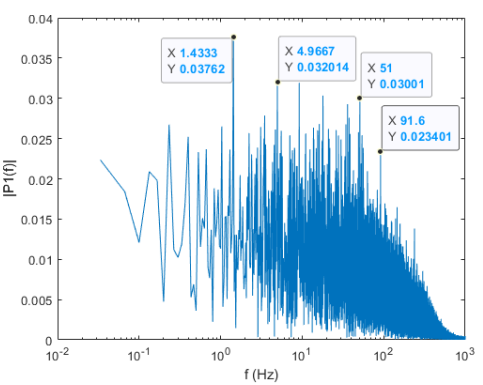

(a) $-(x / d=32.2, y / d=0)$

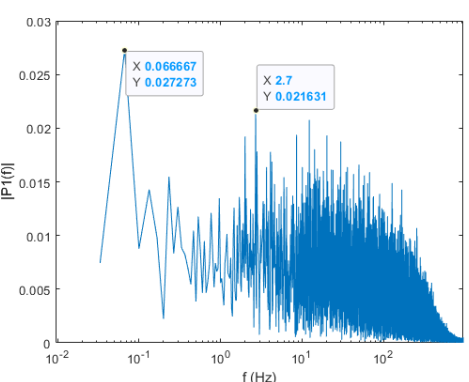

(b) $-(\mathrm{x} / \mathrm{d}=32.2, \mathrm{y} / \mathrm{d}=0)$

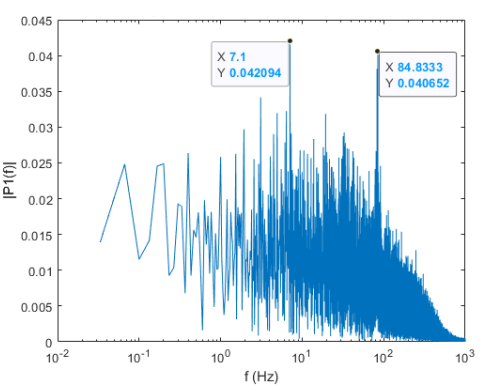

(c) $-(\mathrm{x} / \mathrm{d}=32.2, \mathrm{y} / \mathrm{d}=0)$

Figure A.2. Single sided frequency spectrum at Reynold Number 500 and incident angle (a) $0^{0}$ (b) $45^{\circ}$ and (c) $180^{\circ}$ 


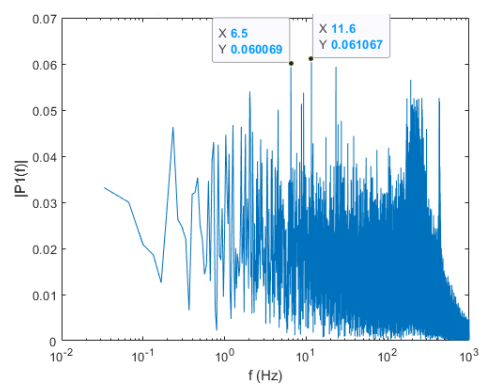

(a) $-(x / d=1.61, y / d=0)$

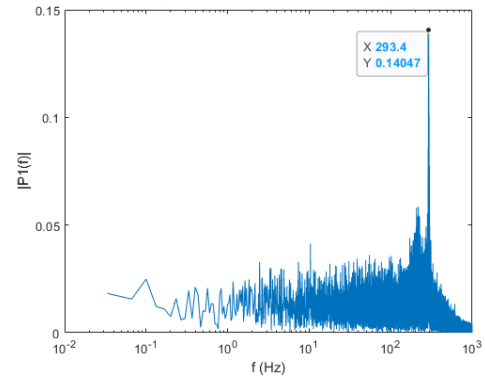

(b) $-(\mathrm{x} / \mathrm{d}=1.61, \mathrm{y} / \mathrm{d}=0)$

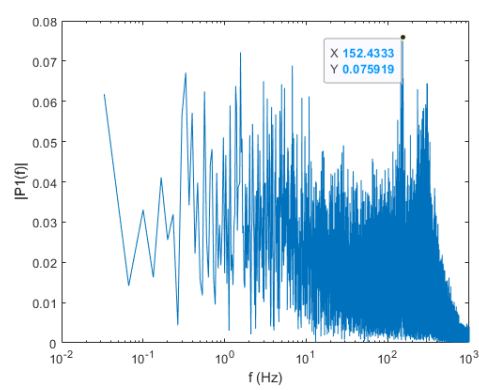

(c) $-(\mathrm{x} / \mathrm{d}=1.61, \mathrm{y} / \mathrm{d}=0)$

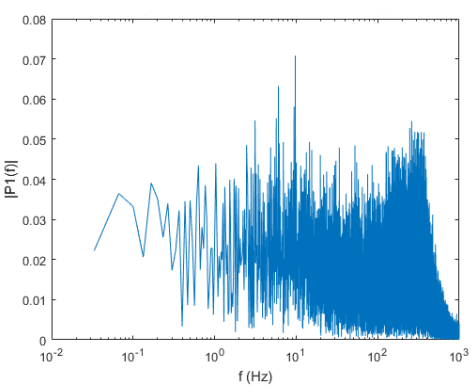

(a) $-(x / d=1.61, y / d=2.21)$

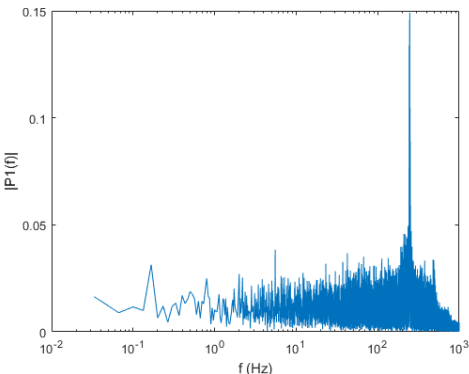

(b) $-(x / d=1.61, y / d=2.21)$

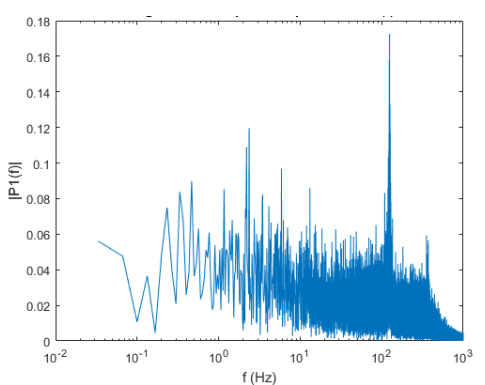

(c) $-(\mathrm{x} / \mathrm{d}=1.61, \mathrm{y} / \mathrm{d}=2.21)$

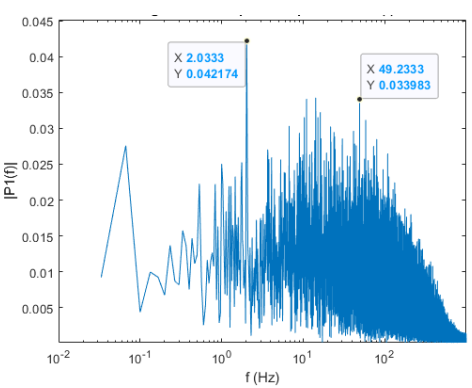

(a) $-(\mathrm{x} / \mathrm{d}=32.2, \mathrm{y} / \mathrm{d}=0)$

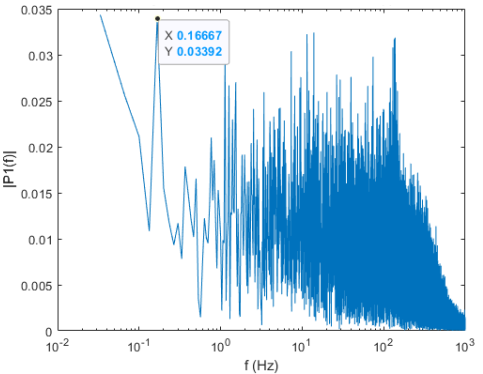

(b) $-(\mathrm{x} / \mathrm{d}=32.2, \mathrm{y} / \mathrm{d}=0)$

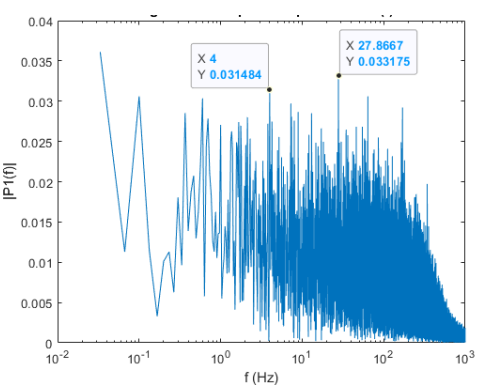

(c) $-(\mathrm{x} / \mathrm{d}=32.2, \mathrm{y} / \mathrm{d}=0)$

Figure A.3. Single sided frequency spectrum at Reynold Number 858 and incident angle (a) $0^{0}$ (b) $45^{\circ}$ and (c) $180^{\circ}$ 


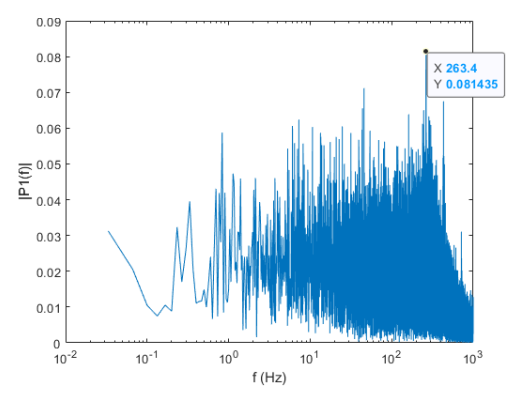

(a) $-(\mathrm{x} / \mathrm{d}=1.61, \mathrm{y} / \mathrm{d}=0)$

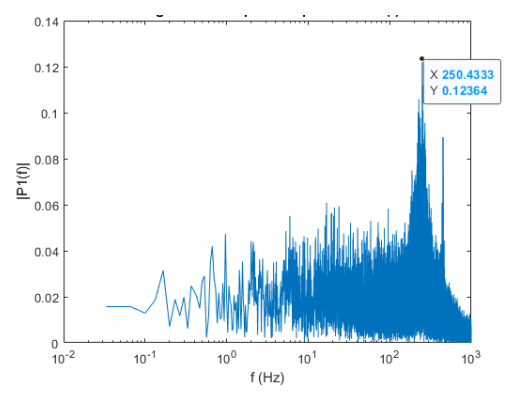

(b) $-(x / d=1.61, y / d=0)$

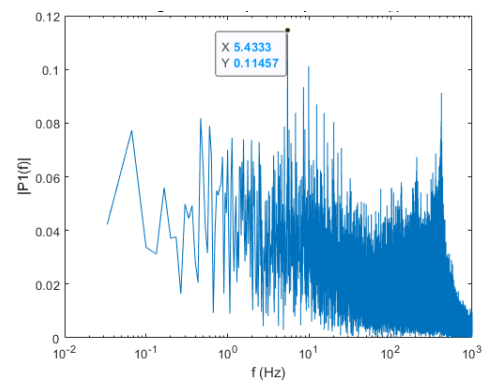

(c) $-(\mathrm{x} / \mathrm{d}=1.61, \mathrm{y} / \mathrm{d}=0)$

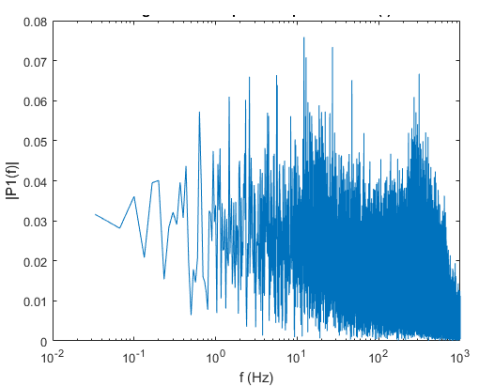

(a) $-(x / d=1.61, y / d=2.21)$

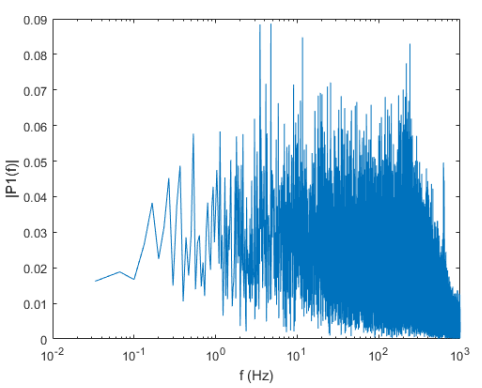

(b) $-(x / d=1.61, y / d=2.21)$

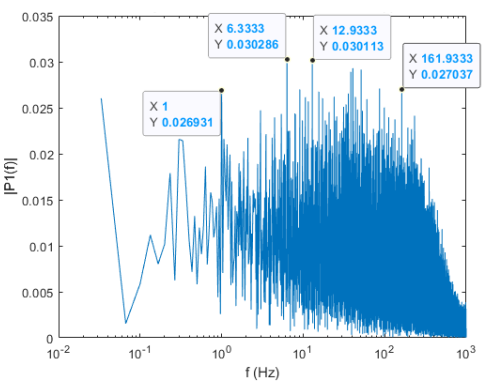

(b) $-(\mathrm{x} / \mathrm{d}=32.2, \mathrm{y} / \mathrm{d}=0)$
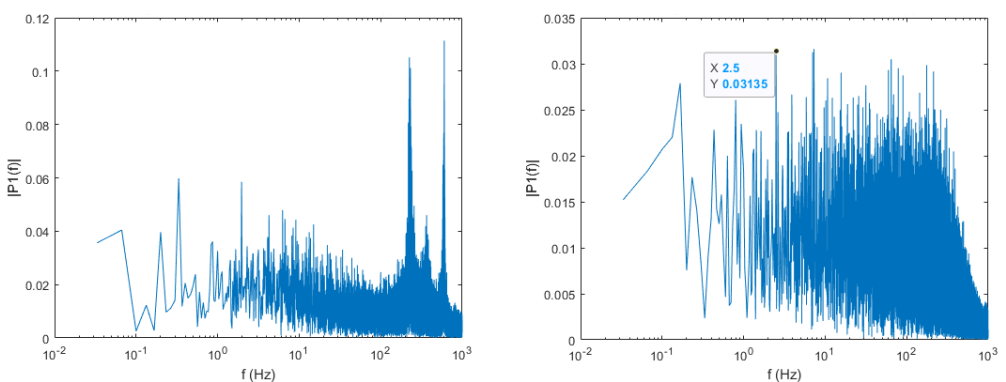

(c) $-(\mathrm{x} / \mathrm{d}=32.2, \mathrm{y} / \mathrm{d}=0)$

Figure A.4. Single sided frequency spectrum at Reynold Number 1250 and incident angle (a) $0^{0}$ (b) $45^{\circ}$ and (c) $180^{\circ}$ 


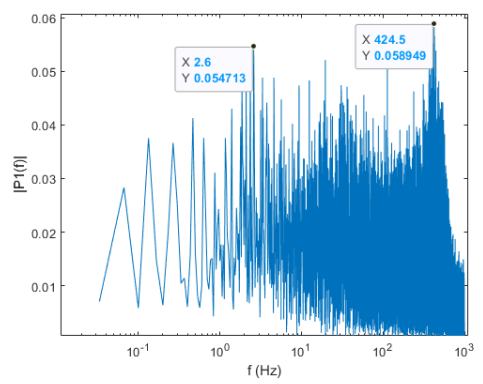

(a) $-(\mathrm{x} / \mathrm{d}=1.61, \mathrm{y} / \mathrm{d}=0)$

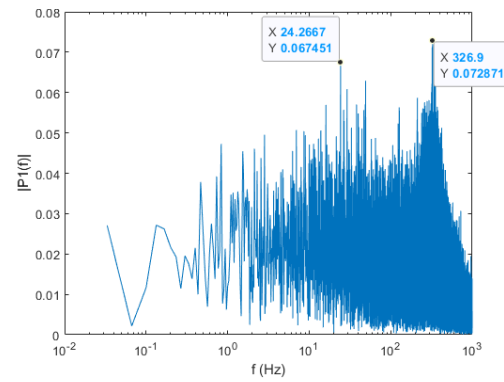

(b) $-(\mathrm{x} / \mathrm{d}=1.61, \mathrm{y} / \mathrm{d}=0)$

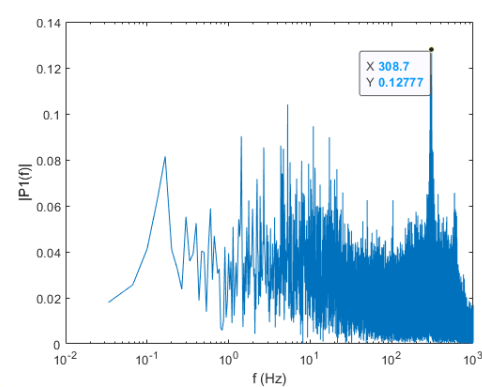

(c) $-(\mathrm{x} / \mathrm{d}=1.61, \mathrm{y} / \mathrm{d}=0)$

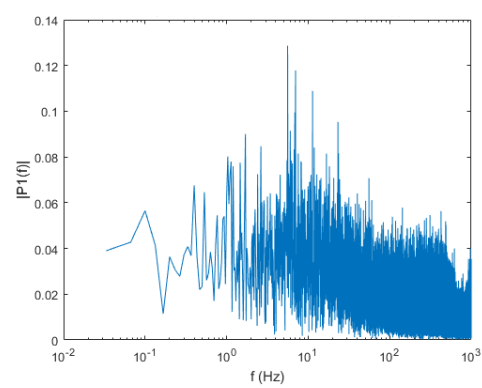

(a) $-(x / d=1.61, y / d=2.21)$

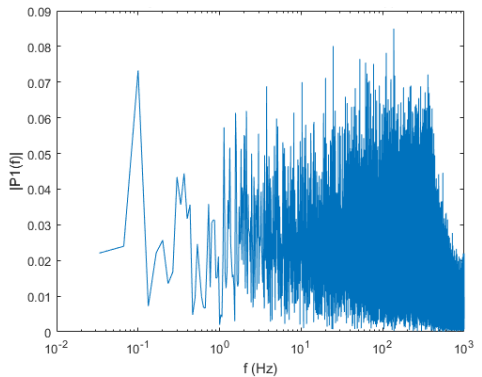

(b) $-(x / d=1.61, y / d=2.21)$

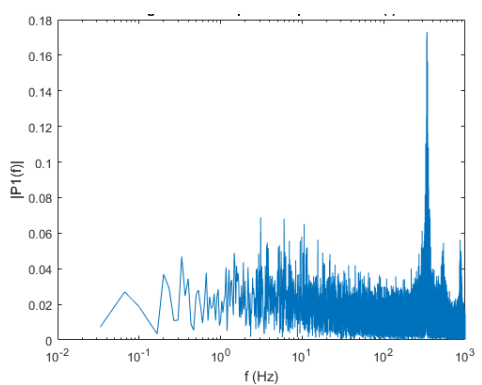

(c) $-(x / d=1.61, y / d=2.21)$

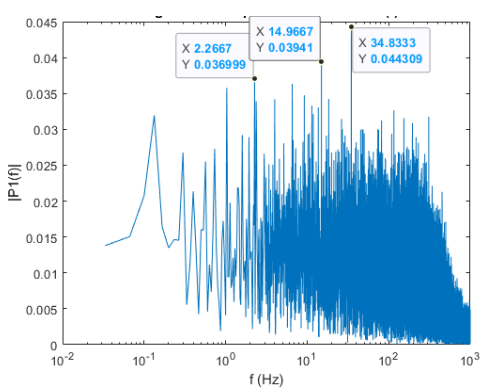

(a) $-(x / d=32.2, y / d=0)$

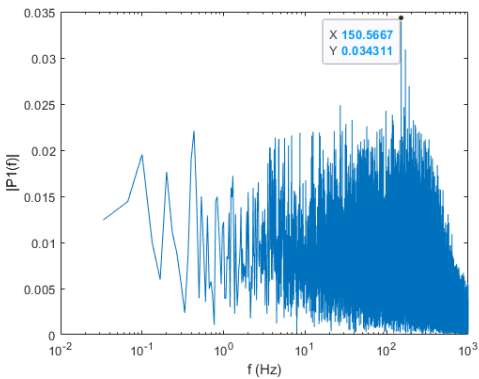

(b) $-(\mathrm{x} / \mathrm{d}=32.2, \mathrm{y} / \mathrm{d}=0)$

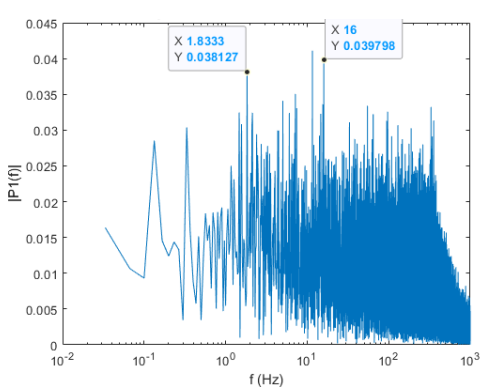

(c) $-(\mathrm{x} / \mathrm{d}=32.2, \mathrm{y} / \mathrm{d}=0)$

Figure A.5. Single sided frequency spectrum at Reynold Number 1700 and incident angle (a) $0^{0}$ (b) $45^{\circ}$ and (c) $180^{\circ}$ 


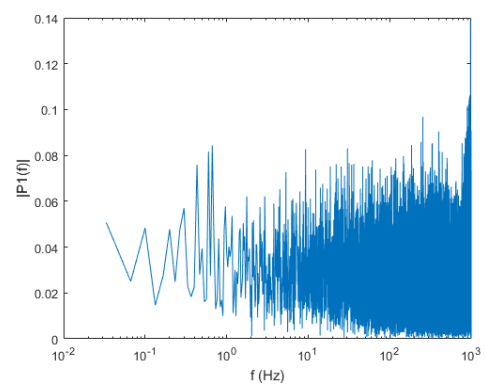

(a) $-(x / d=1.61, y / d=0)$

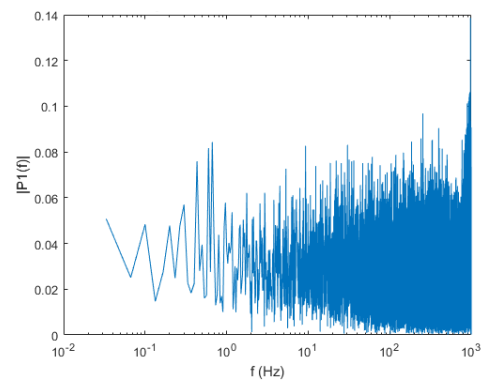

(a) $-(x / d=1.61, y / d=0)$

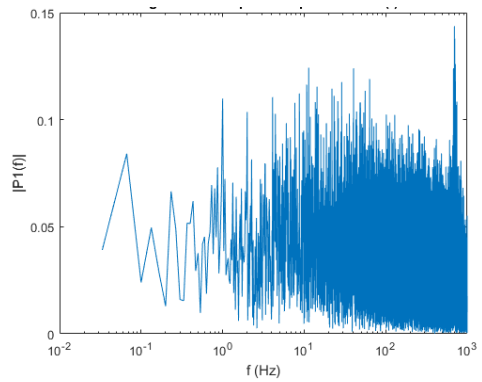

(a) $-(x / d=1.61, y / d=0)$

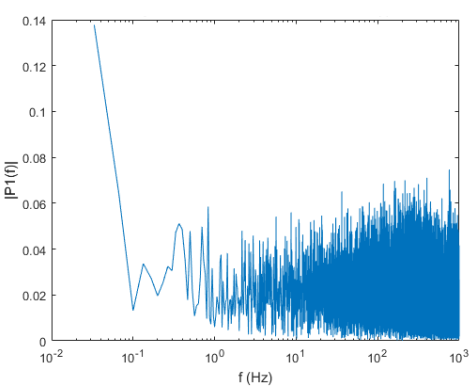

(a) $-(x / d=1.61, y / d=2.21)$

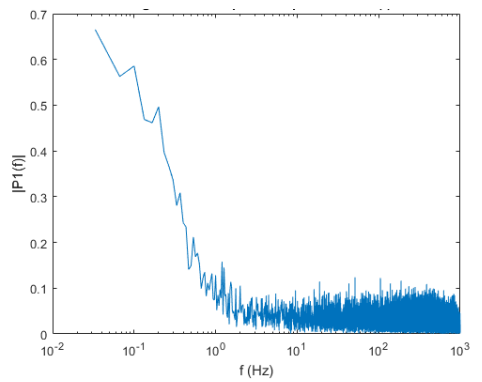

(a) $-(x / d=1.61, y / d=2.21)$

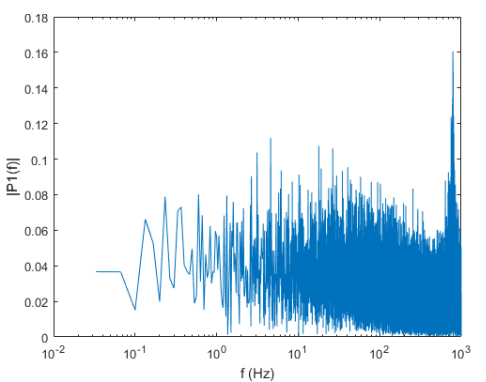

(a) $-(x / d=1.61, y / d=2.21)$

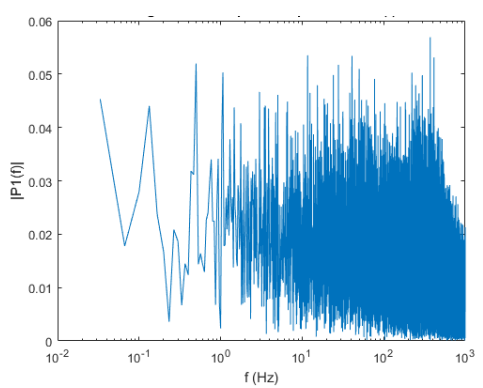

(a) $-(x / d=32.2, y / d=0)$

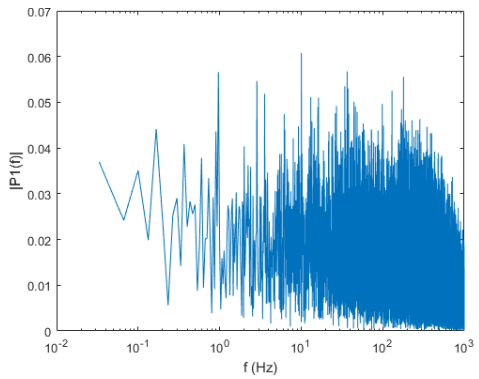

(a) $-(x / d=32.2, y / d=0)$

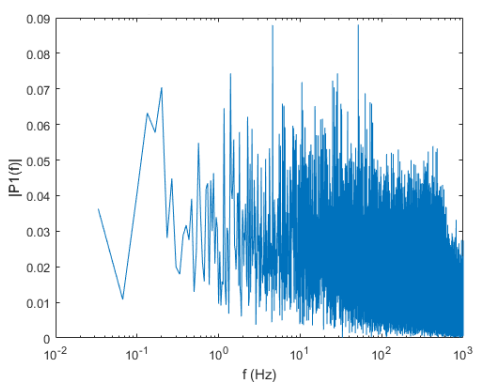

(a) $-(x / d=32.2, y / d=0)$

Figure A.6. Single sided frequency spectrum at Reynold Number 4350 and incident angle (a) $0^{0}$ (b) $45^{\circ}$ and (c) $180^{\circ}$ 


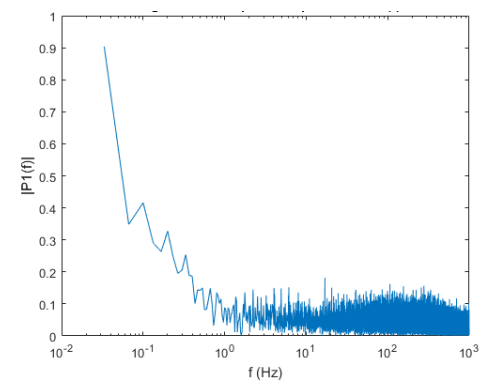

(a) $-(\mathrm{x} / \mathrm{d}=1.61, \mathrm{y} / \mathrm{d}=0)$

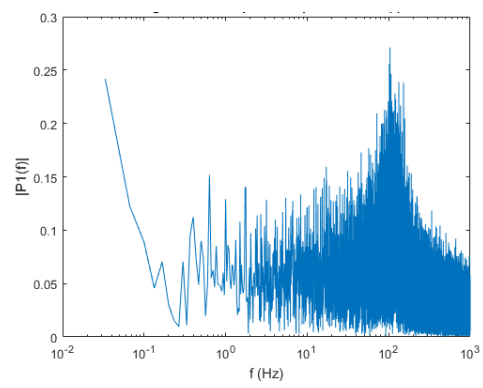

(b) $-(\mathrm{x} / \mathrm{d}=1.61, \mathrm{y} / \mathrm{d}=0)$

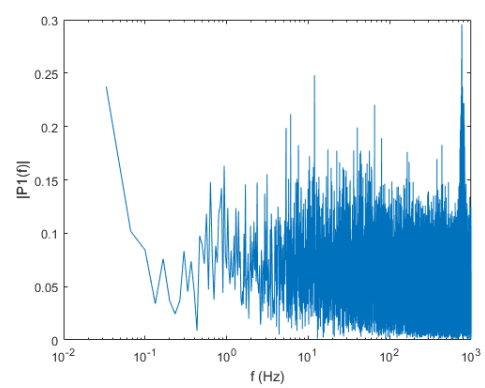

(c) $-(\mathrm{x} / \mathrm{d}=1.61, \mathrm{y} / \mathrm{d}=0)$

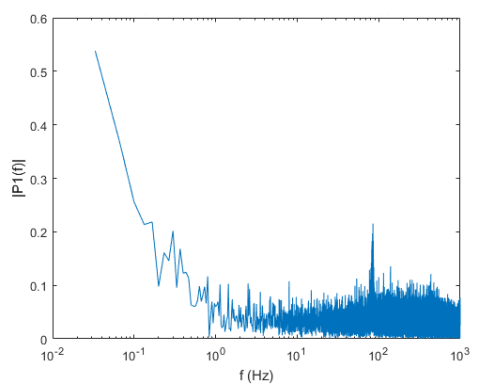

(a) $-(\mathrm{x} / \mathrm{d}=1.61, \mathrm{y} / \mathrm{d}=2.21)$

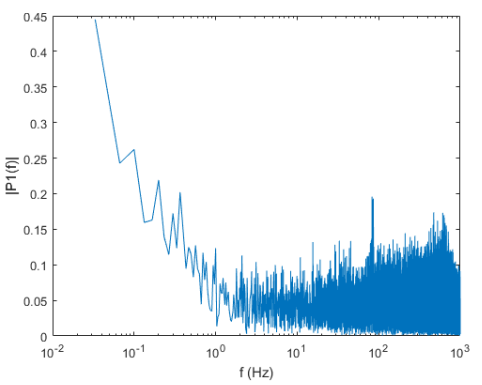

(b) $-(\mathrm{x} / \mathrm{d}=1.61, \mathrm{y} / \mathrm{d}=2.21)$

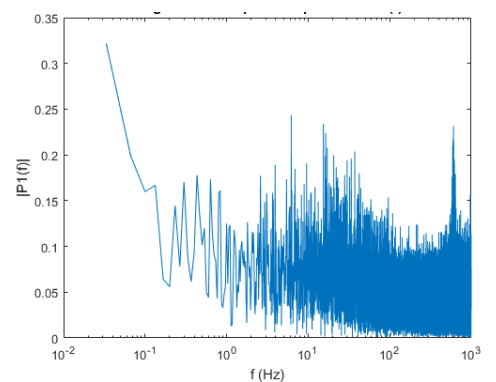

(c) $-(\mathrm{x} / \mathrm{d}=1.61, \mathrm{y} / \mathrm{d}=2.21)$

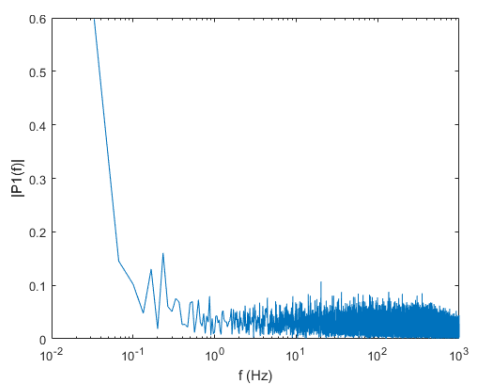

(a) $-(\mathrm{x} / \mathrm{d}=32.2, \mathrm{y} / \mathrm{d}=0)$

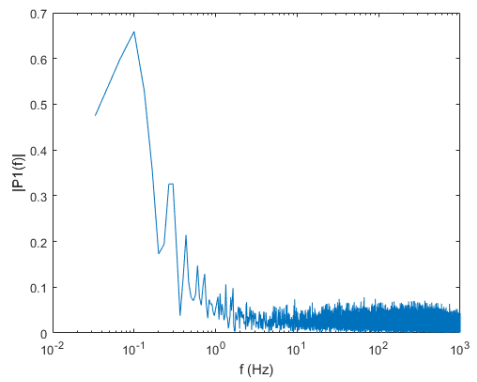

(b) $-(\mathrm{x} / \mathrm{d}=32.2, \mathrm{y} / \mathrm{d}=0)$

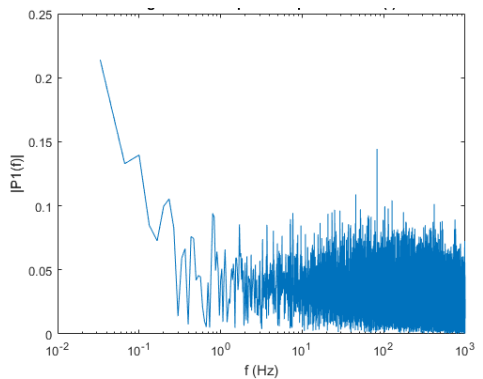

(c) $-(\mathrm{x} / \mathrm{d}=32.2, \mathrm{y} / \mathrm{d}=0)$

Figure A.7. Single sided frequency spectrum at Reynold Number 7470 and incident angle (a) $0^{0}$ (b) $45^{\circ}$ and (c) $180^{\circ}$ 


\section{APPENDIX B}

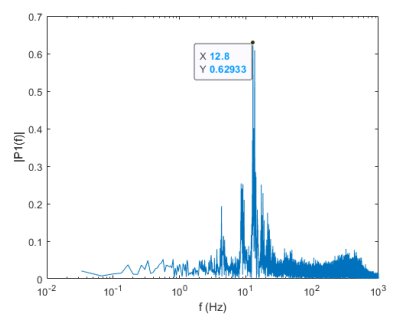

(a) $-(x / d=1.61, y / d=0)$

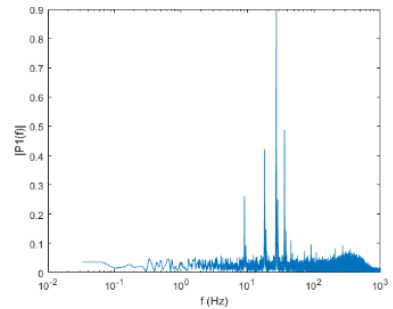

(b) $-(x / d=1.61, y / d=0)$

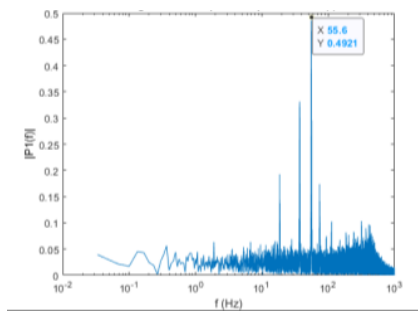

(c) $-(\mathrm{x} / \mathrm{d}=1.61, \mathrm{y} / \mathrm{d}=0)$

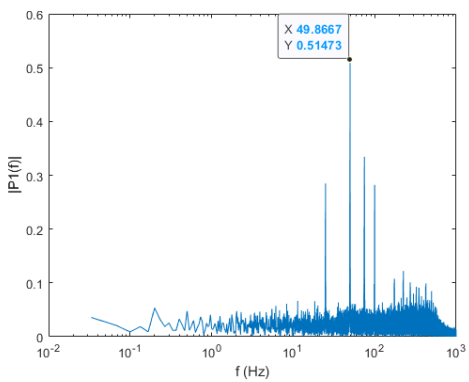

(d) $-(x / d=1.61, y / d=0)$

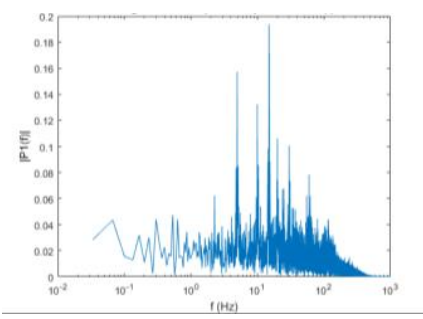

(a) $-(x / d=1.61, y / d=2.21)$

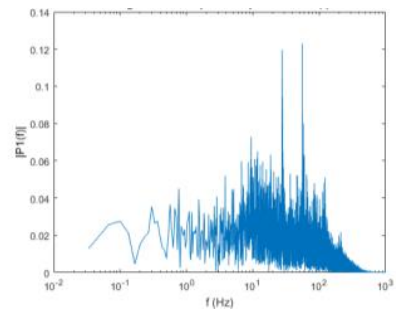

(b) $-(x / d=1.61, y / d=2.21)$

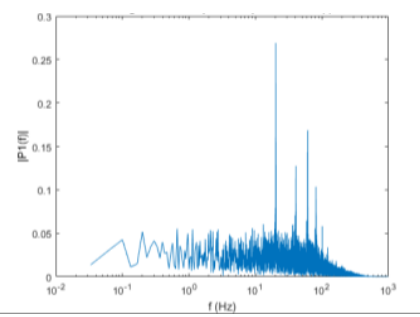

(c) $-(\mathrm{x} / \mathrm{d}=1.61, \mathrm{y} / \mathrm{d}=2.21)$

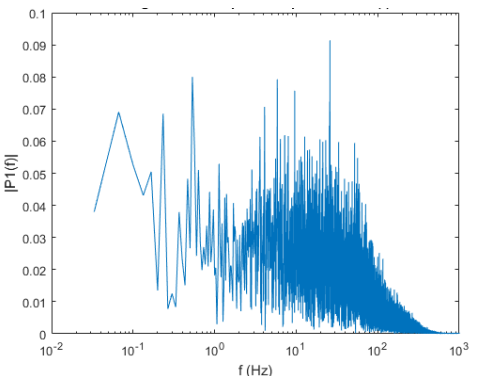

(d) $-(x / d=1.61, y / d=2.21)$

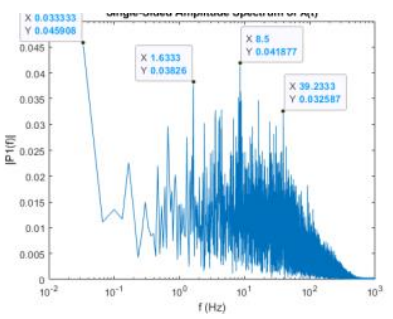

(a) $-(\mathrm{x} / \mathrm{d}=32.2, \mathrm{y} / \mathrm{d}=0)$

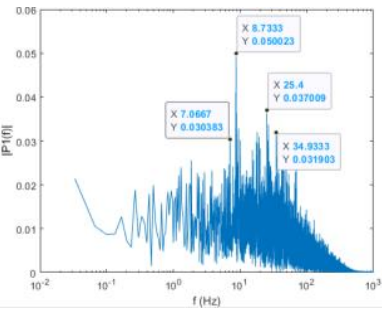

(b) $-(\mathrm{x} / \mathrm{d}=32.2, \mathrm{y} / \mathrm{d}=0)$

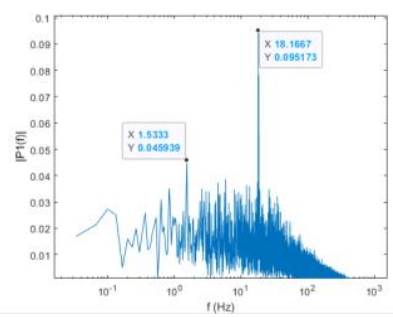

(c) $-(\mathrm{x} / \mathrm{d}=32.2, \mathrm{y} / \mathrm{d}=0)$

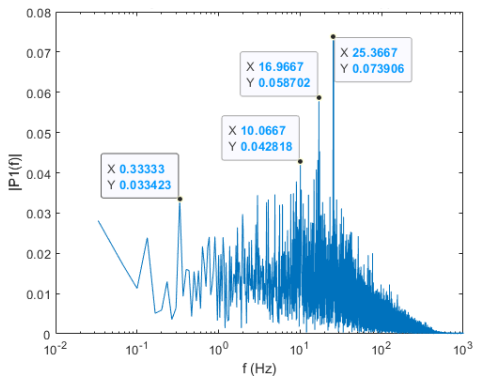

(d) $-(x / d=32.2, y / d=0)$

Figure B.1. Single sided frequency spectrum at Reynold Number 57 and Rotation RPM (a) 120 (b) 360 (c) 960 (d) 1500 (e) 1800 (f) 2400 (g) 3000 RPM 


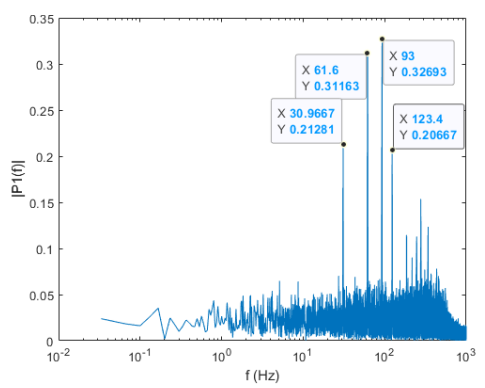

(e) $-(\mathrm{x} / \mathrm{d}=1.61, \mathrm{y} / \mathrm{d}=0)$

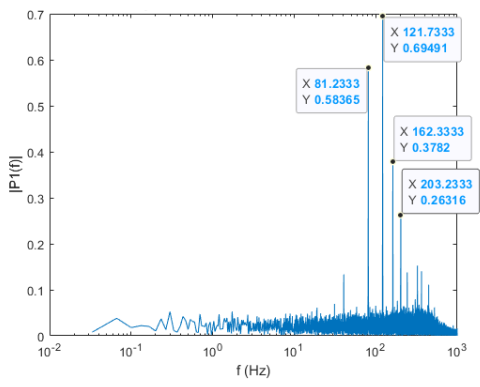

(f) $-(\mathrm{x} / \mathrm{d}=1.61, \mathrm{y} / \mathrm{d}=0)$

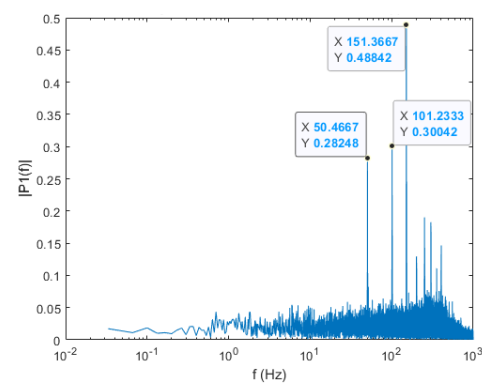

$(\mathrm{g})-(\mathrm{x} / \mathrm{d}=1.61, \mathrm{y} / \mathrm{d}=0)$

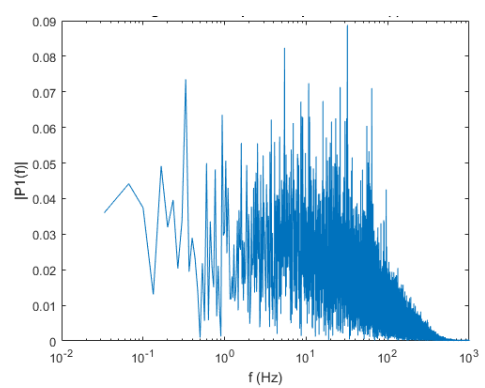

(e) $-(\mathrm{x} / \mathrm{d}=1.61, \mathrm{y} / \mathrm{d}=2.21)$

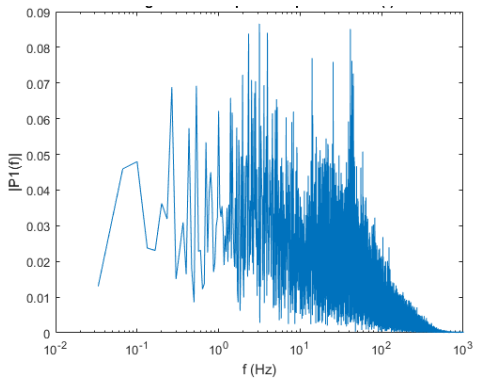

(f) $-(\mathrm{x} / \mathrm{d}=1.61, \mathrm{y} / \mathrm{d}=2.21)$

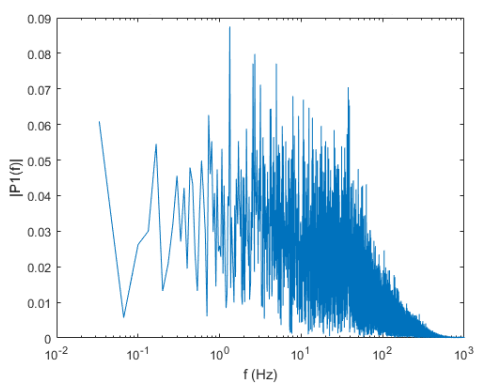

$(\mathrm{g})-(\mathrm{x} / \mathrm{d}=1.61, \mathrm{y} / \mathrm{d}=2.21)$

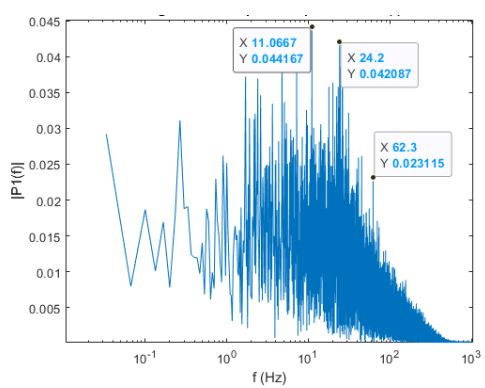

(e) $-(\mathrm{x} / \mathrm{d}=32.2, \mathrm{y} / \mathrm{d}=0)$

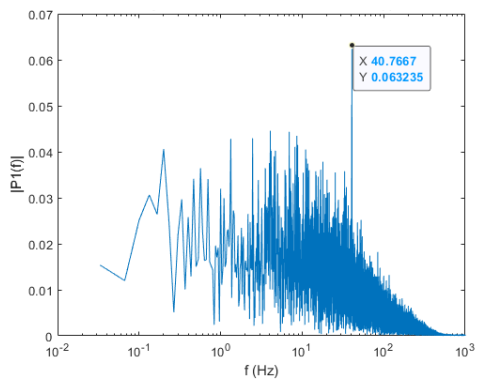

(f) $-(\mathrm{x} / \mathrm{d}=32.2, \mathrm{y} / \mathrm{d}=0)$

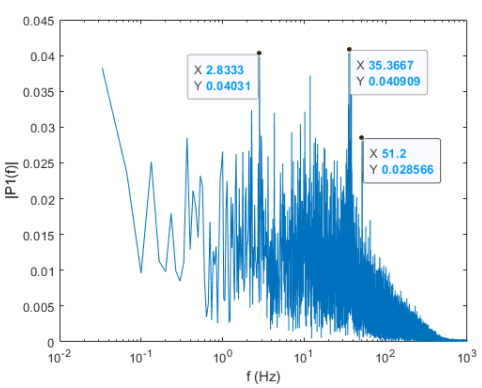

$(\mathrm{g})-(\mathrm{x} / \mathrm{d}=32.2, \mathrm{y} / \mathrm{d}=0)$

Figure B.1. Single sided frequency spectrum at Reynold Number 57 and Rotation RPM (a) 120 (b) 360 (c) 960 (d) 1500 (e) 1800 (f) 2400 (g) 3000 RPM (Continued) 


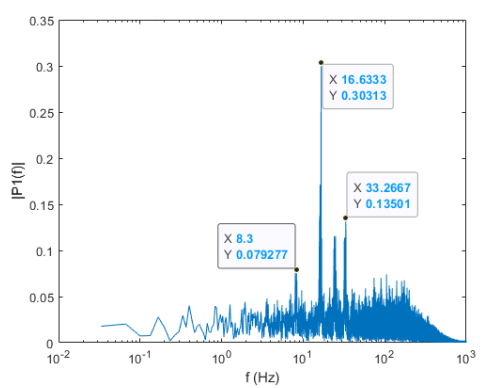

(a) $-(x / d=1.61, y / d=0)$

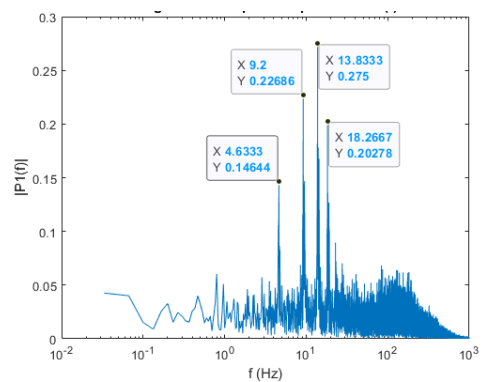

(b) $-(\mathrm{x} / \mathrm{d}=1.61, \mathrm{y} / \mathrm{d}=0)$

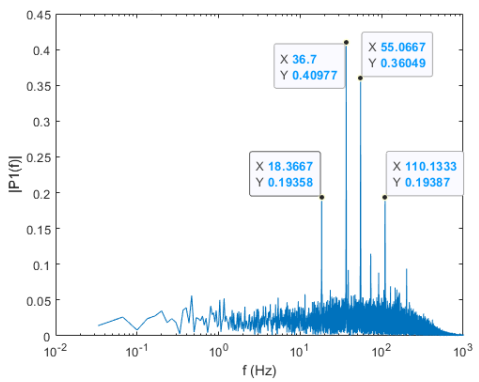

(c) $-(\mathrm{x} / \mathrm{d}=1.61, \mathrm{y} / \mathrm{d}=0)$

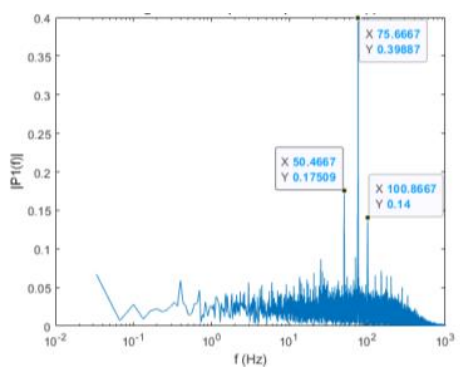

(d) $-(x / d=1.61, y / d=0)$

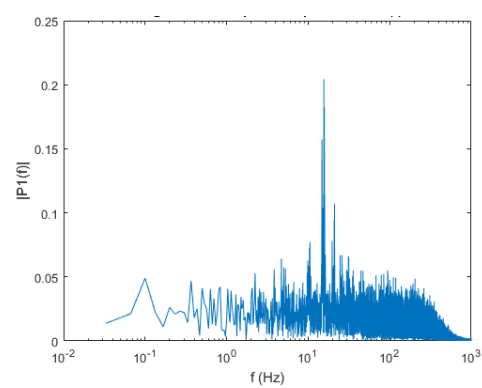

(a) $-(x / d=1.61, y / d=2.21)$

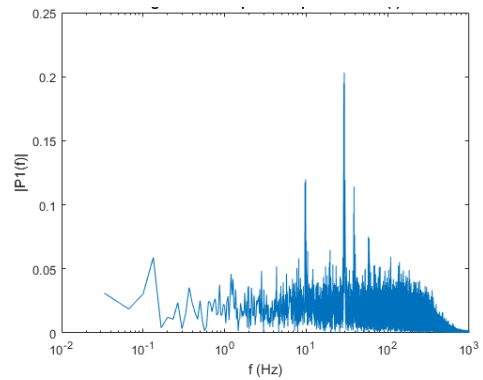

(b) $-(\mathrm{x} / \mathrm{d}=1.61, \mathrm{y} / \mathrm{d}=2.21)$

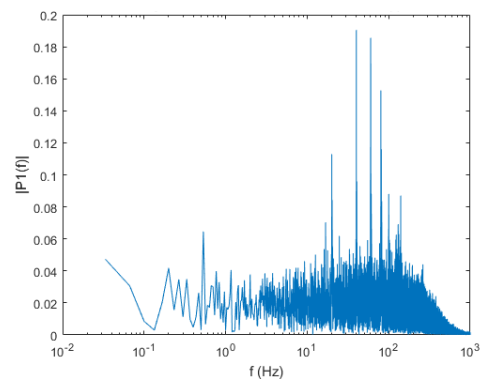

(c) $-(x / d=1.61, y / d=2.21)$

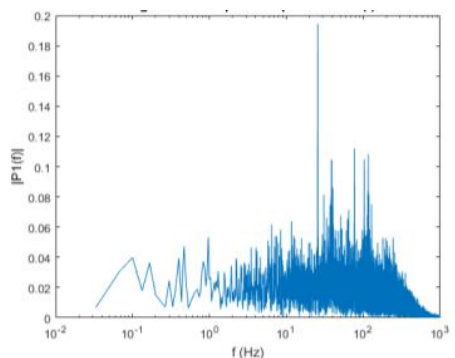

(d) $-(x / d=1.61, y / d=2.21)$

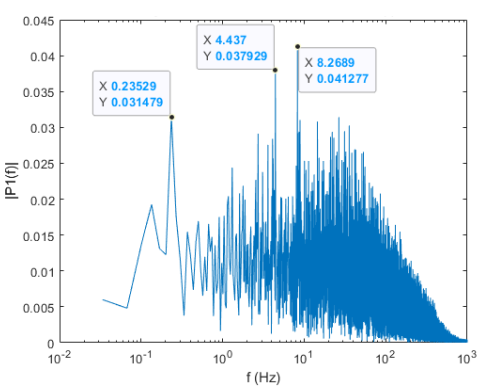

(a) $-(x / d=32.2, y / d=0)$

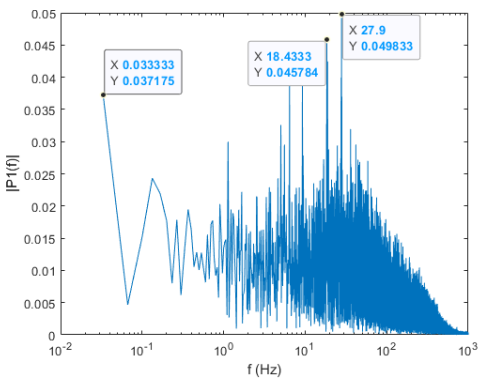

(b) $-(\mathrm{x} / \mathrm{d}=32.2, \mathrm{y} / \mathrm{d}=0)$

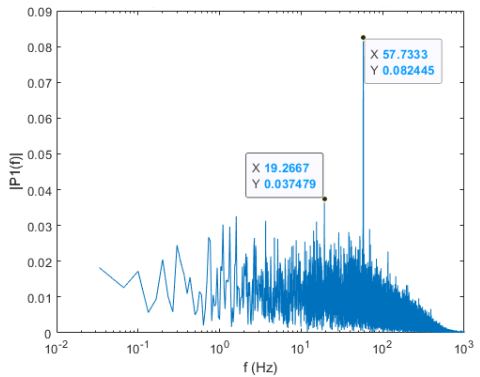

(c) $-(\mathrm{x} / \mathrm{d}=32.2, \mathrm{y} / \mathrm{d}=0)$

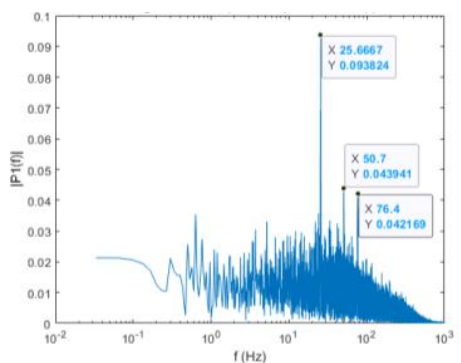

(d) $-(x / d=32.2, y / d=0)$

Figure B.2. Single sided frequency spectrum at Reynold Number 500 and Rotation RPM (a) 120 (b) 360 (c) 960 (d) 1500 (e) 1800 (f) 2400 (g) 3000 RPM 


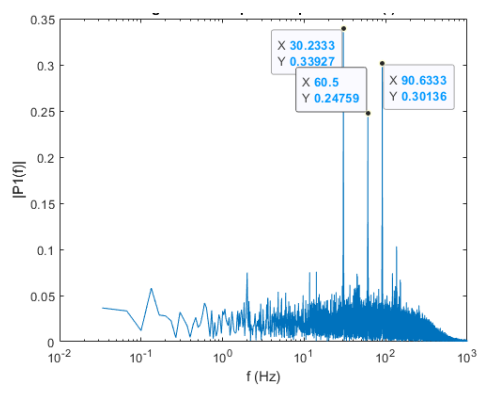

(e) $-(x / d=1.61, y / d=0)$

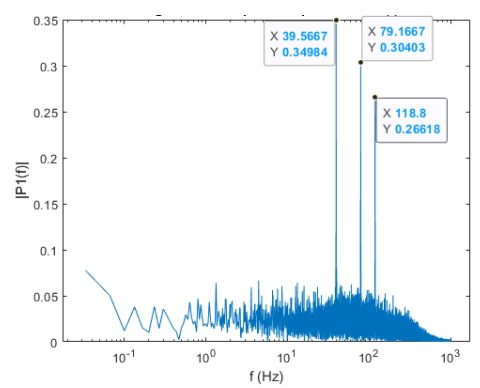

(f) $-(\mathrm{x} / \mathrm{d}=1.61, \mathrm{y} / \mathrm{d}=0)$

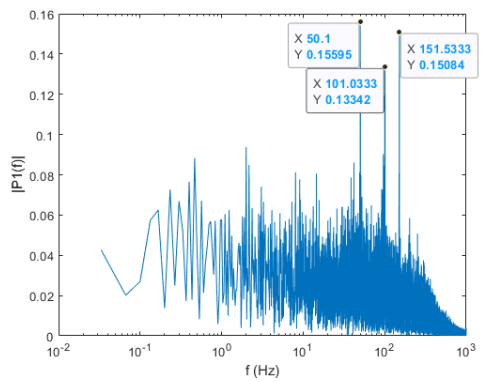

$(\mathrm{g})-(\mathrm{x} / \mathrm{d}=1.61, \mathrm{y} / \mathrm{d}=0)$

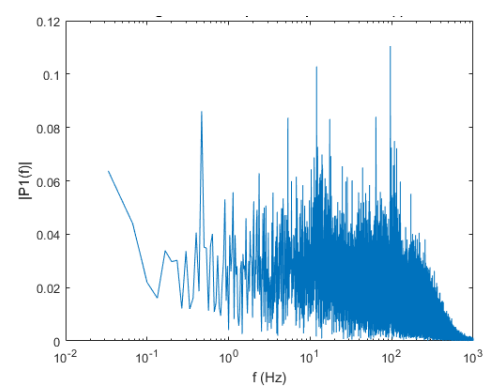

(e) $-(x / d=1.61, y / d=2.21)$

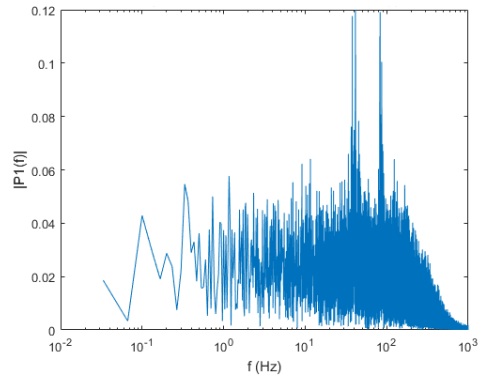

(f) $-(\mathrm{x} / \mathrm{d}=1.61, \mathrm{y} / \mathrm{d}=2.21)$

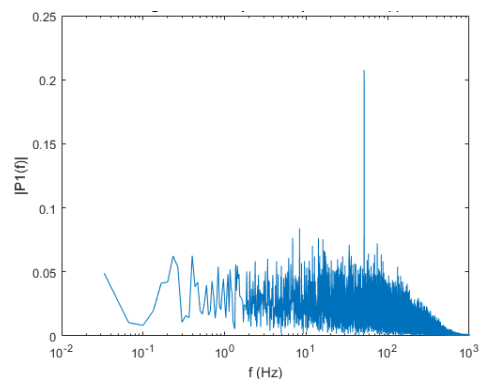

$(\mathrm{g})-(\mathrm{x} / \mathrm{d}=1.61, \mathrm{y} / \mathrm{d}=2.21)$

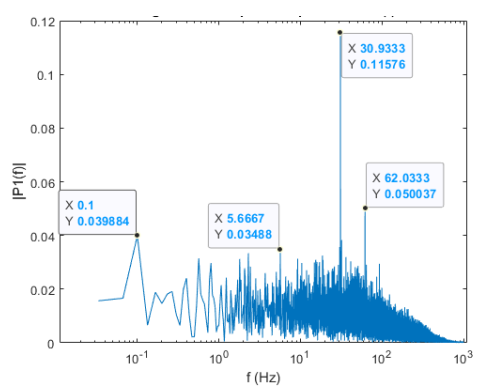

(e) $-(x / d=32.2, y / d=0)$

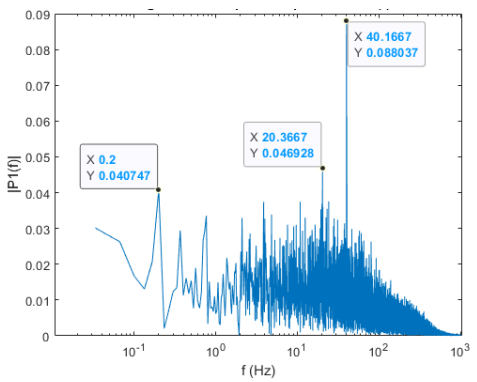

(f) $-(\mathrm{x} / \mathrm{d}=32.2, \mathrm{y} / \mathrm{d}=0)$

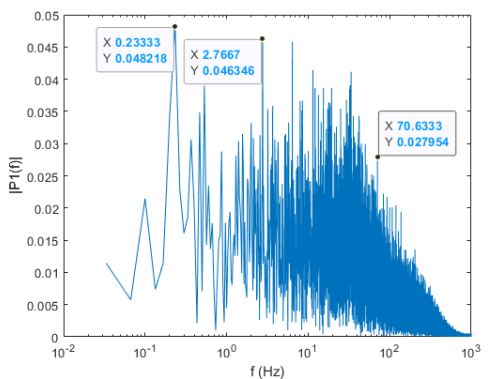

$(\mathrm{g})-(\mathrm{x} / \mathrm{d}=32.2, \mathrm{y} / \mathrm{d}=0)$

Figure B.2. Single sided frequency spectrum at Reynold Number 500 and Rotation RPM (a) 120 (b) 360 (c) 960 (d) 1500 (e) 1800 (f) 2400 (g) 3000 RPM (Continued) 


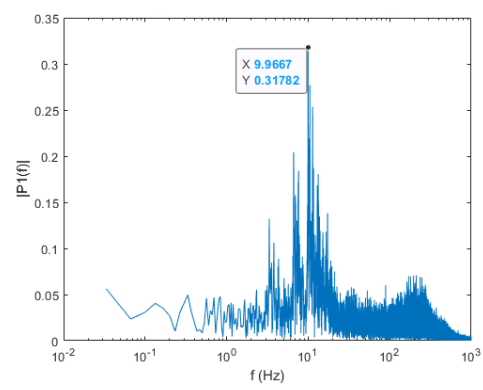

(a) $-(x / d=1.61, y / d=0)$

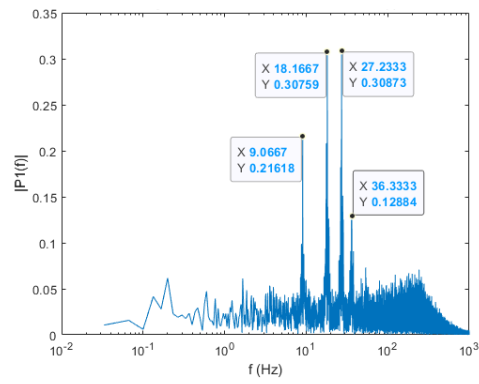

(b) $-(\mathrm{x} / \mathrm{d}=1.61, \mathrm{y} / \mathrm{d}=0)$

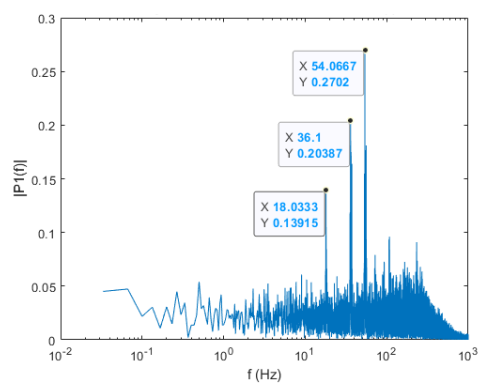

(c) $-(\mathrm{x} / \mathrm{d}=1.61, \mathrm{y} / \mathrm{d}=0)$

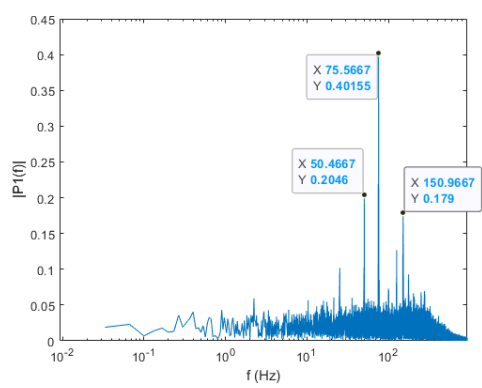

(d) $-(\mathrm{x} / \mathrm{d}=1.61, \mathrm{y} / \mathrm{d}=0)$

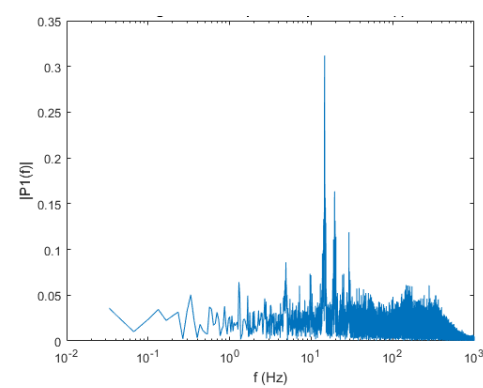

(a) $-(x / d=1.61, y / d=2.21)$

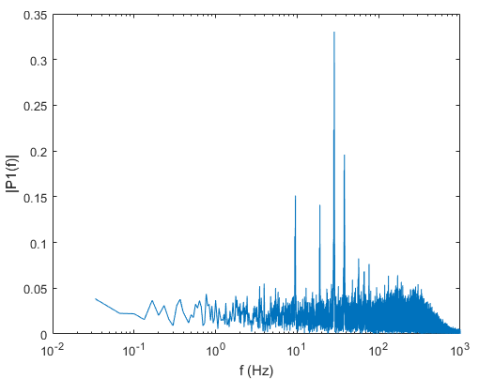

(b) $-(\mathrm{x} / \mathrm{d}=1.61, \mathrm{y} / \mathrm{d}=2.21)$

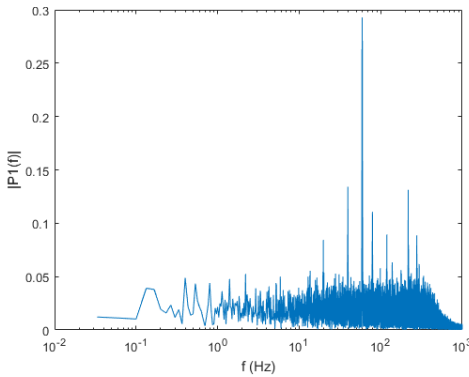

(c) $-(x / d=1.61, y / d=2.21)$

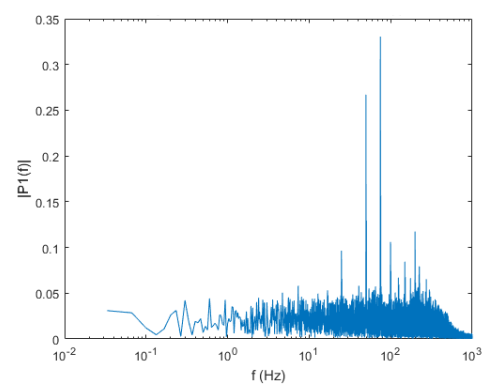

(d) $-(x / d=1.61, y / d=2.21)$

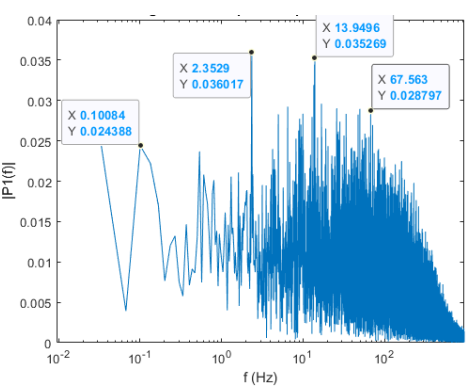

(a) $-(x / d=32.2, y / d=0)$

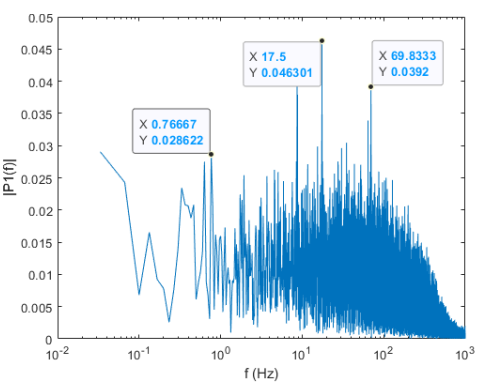

(b) $-(\mathrm{x} / \mathrm{d}=32.2, \mathrm{y} / \mathrm{d}=0)$

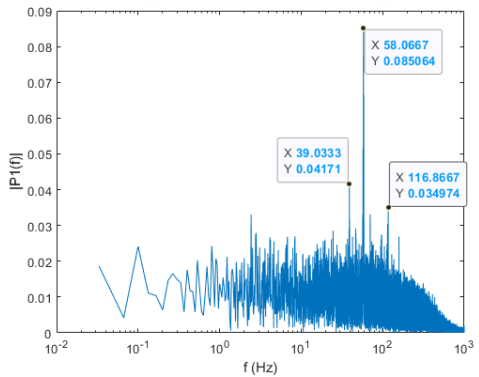

(c) $-(\mathrm{x} / \mathrm{d}=32.2, \mathrm{y} / \mathrm{d}=0)$

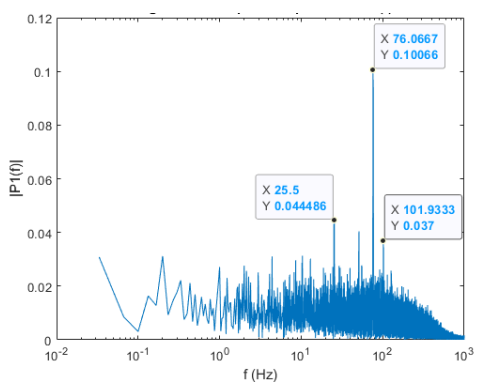

(d) $-(\mathrm{x} / \mathrm{d}=32.2, \mathrm{y} / \mathrm{d}=0)$

Figure B.3. Single sided frequency spectrum at Reynold Number 858 and Rotation RPM (a) 120 (b) 360 (c) 960 (d) 1500 (e) 1800 (f) 2400 (g) 3000 RPM 


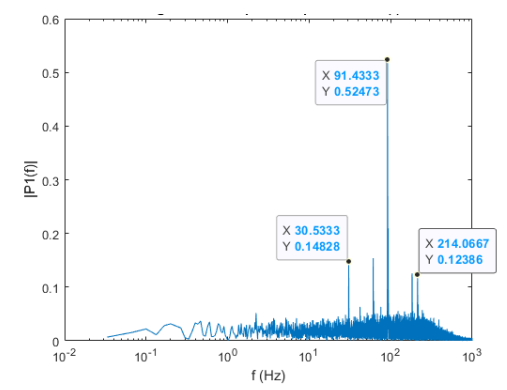

(e) $-(\mathrm{x} / \mathrm{d}=1.61, \mathrm{y} / \mathrm{d}=0)$

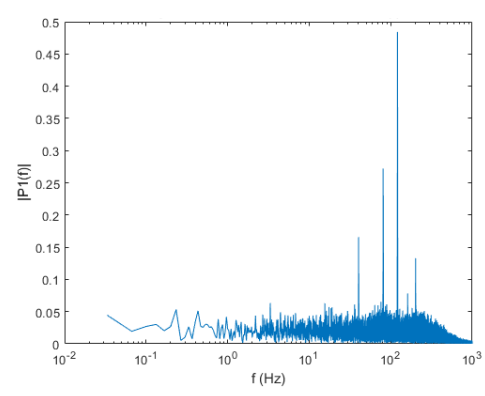

(f) $-(\mathrm{x} / \mathrm{d}=1.61, \mathrm{y} / \mathrm{d}=0)$

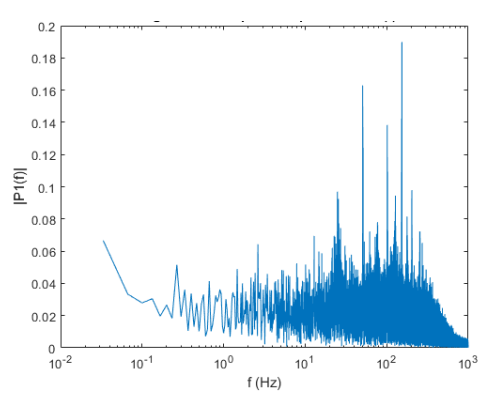

(f) $-(\mathrm{x} / \mathrm{d}=1.61, \mathrm{y} / \mathrm{d}=0)$

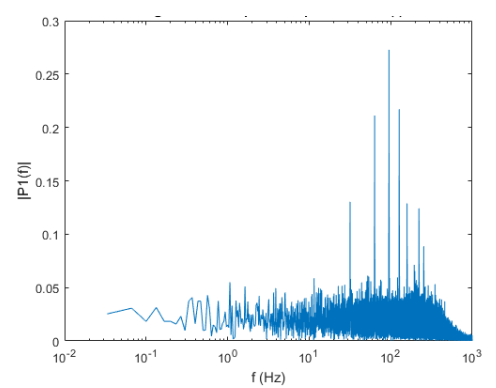

(e) $-(\mathrm{x} / \mathrm{d}=1.61, \mathrm{y} / \mathrm{d}=2.21)$

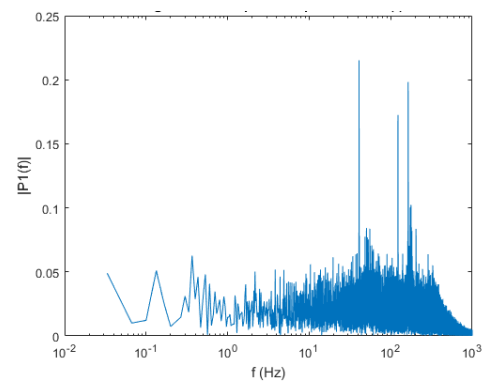

(f) $-(\mathrm{x} / \mathrm{d}=1.61, \mathrm{y} / \mathrm{d}=2.21)$

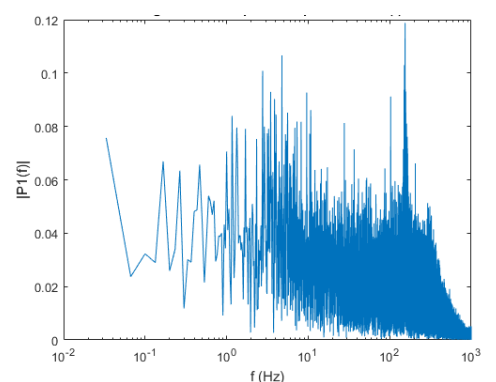

(f) $-(\mathrm{x} / \mathrm{d}=1.61, \mathrm{y} / \mathrm{d}=2.21)$

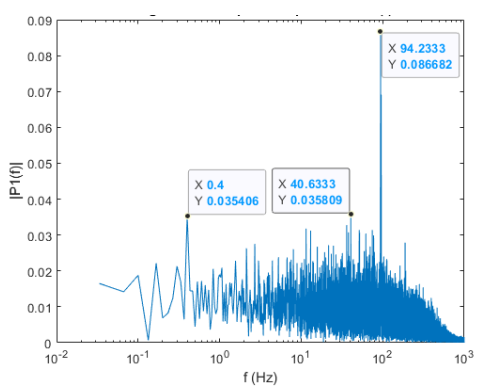

(e) $-(\mathrm{x} / \mathrm{d}=32.2, \mathrm{y} / \mathrm{d}=0)$

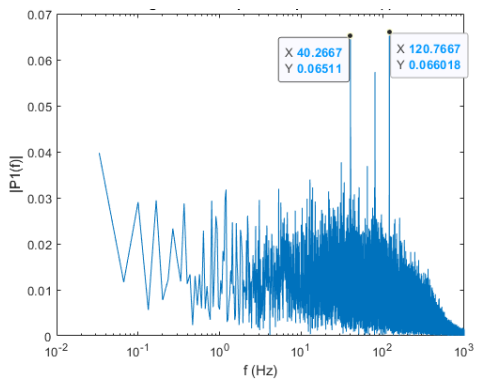

(f) $-(\mathrm{x} / \mathrm{d}=32.2, \mathrm{y} / \mathrm{d}=0)$

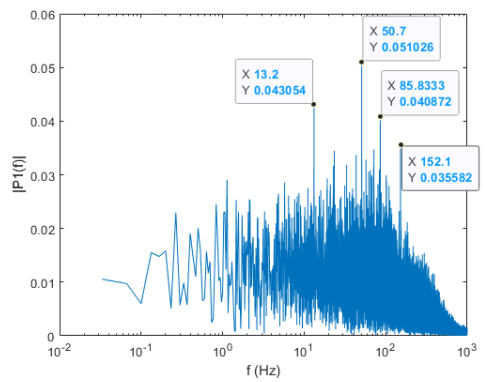

(f) $-(\mathrm{x} / \mathrm{d}=32.2, \mathrm{y} / \mathrm{d}=0)$

Figure B.3. Single sided frequency spectrum at Reynold Number 858 and Rotation RPM (a) 120 (b) 360 (c) 960 (d) 1500 (e) 1800 (f) 2400 (g) 3000 RPM (Continued) 


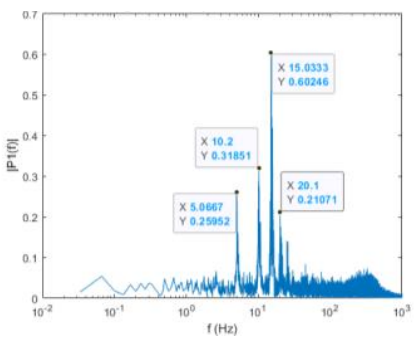

(a) $-(\mathrm{x} / \mathrm{d}=1.61, \mathrm{y} / \mathrm{d}=0)$

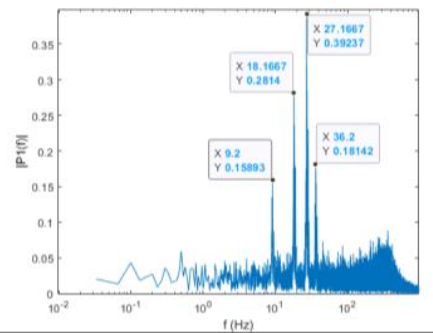

(b) $-(\mathrm{x} / \mathrm{d}=1.61, \mathrm{y} / \mathrm{d}=0)$

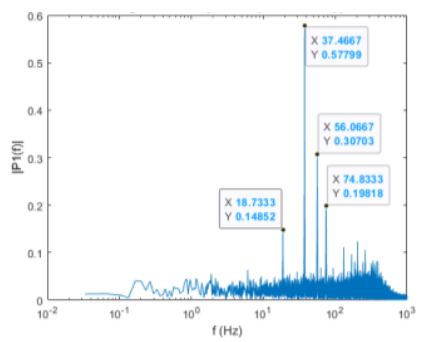

(c) $-(\mathrm{x} / \mathrm{d}=1.61, \mathrm{y} / \mathrm{d}=0)$

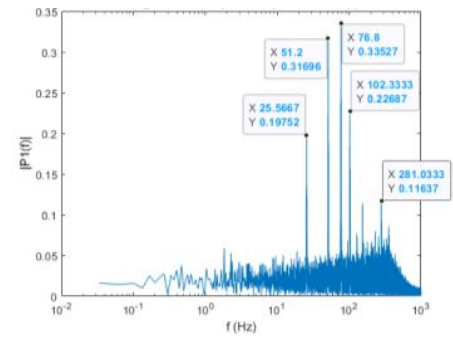

(d) $-(x / d=1.61, y / d=0)$

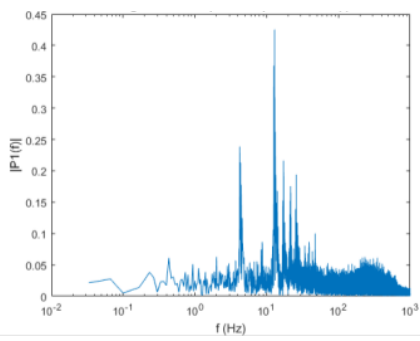

(a) $-(\mathrm{x} / \mathrm{d}=1.61, \mathrm{y} / \mathrm{d}=2.21)$

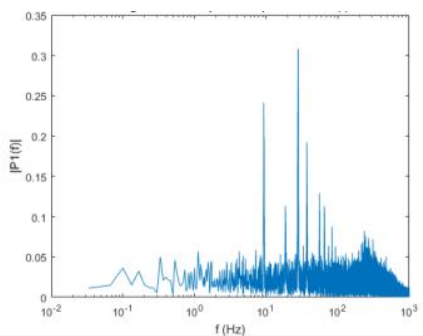

(b) $-(x / d=1.61, y / d=2.21)$

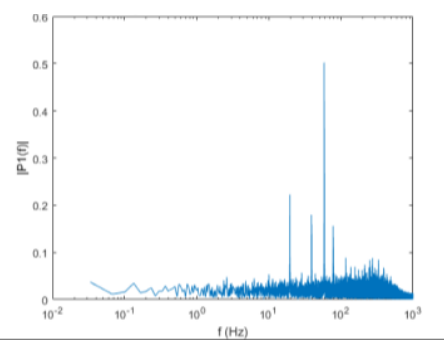

(c) $-(\mathrm{x} / \mathrm{d}=1.61, \mathrm{y} / \mathrm{d}=2.21)$

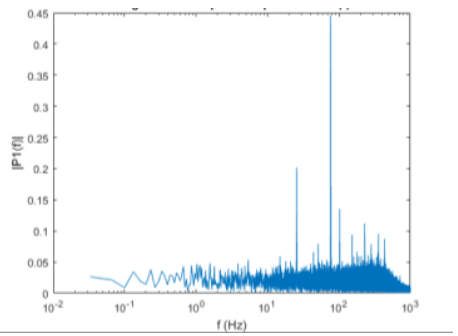

(d) $-(\mathrm{x} / \mathrm{d}=1.61, \mathrm{y} / \mathrm{d}=2.21)$

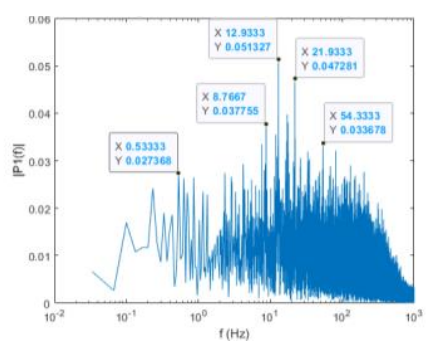

(a) $-(\mathrm{x} / \mathrm{d}=32.2, \mathrm{y} / \mathrm{d}=0)$

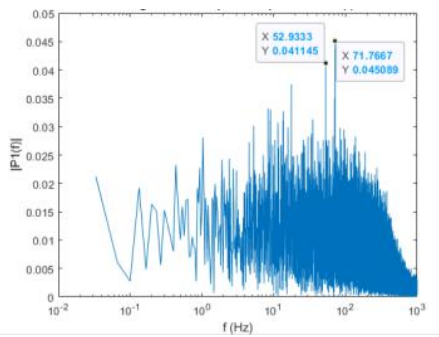

(b) $-(\mathrm{x} / \mathrm{d}=32.2, \mathrm{y} / \mathrm{d}=0)$

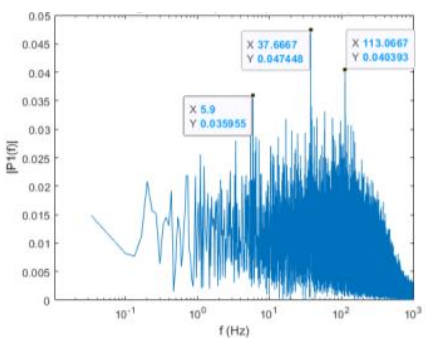

(c) $-(\mathrm{x} / \mathrm{d}=32.2, \mathrm{y} / \mathrm{d}=0)$

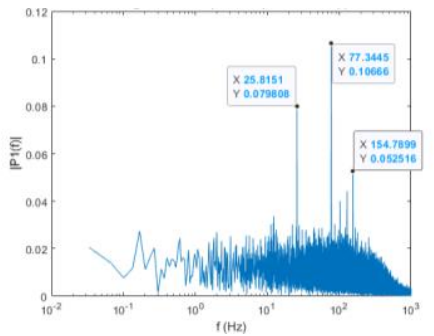

(d) $-(\mathrm{x} / \mathrm{d}=32.2, \mathrm{y} / \mathrm{d}=0)$

Figure B.4. Single sided frequency spectrum at Reynold Number 1250 and Rotation RPM (a) 120 (b) 360 (c) 960 (d) 1500 (e) 1800 (f) 2400 (g) 3000 RPM 


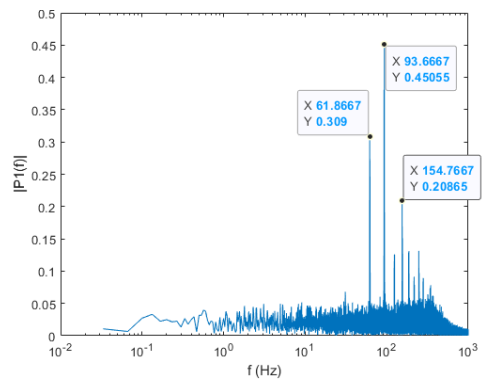

(e) $-(\mathrm{x} / \mathrm{d}=1.61, \mathrm{y} / \mathrm{d}=0)$

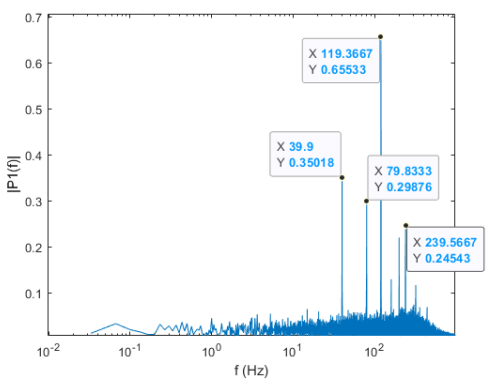

(f)-(x/d=1.61, y/d=0)

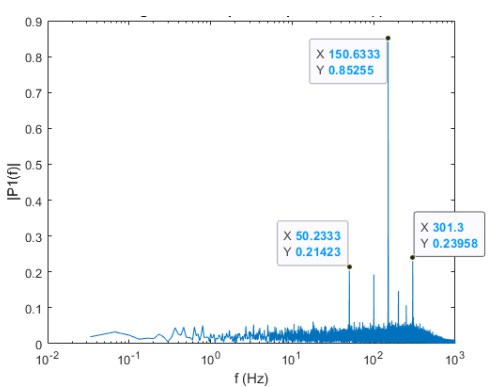

$(\mathrm{g})-(\mathrm{x} / \mathrm{d}=1.61, \mathrm{y} / \mathrm{d}=0)$

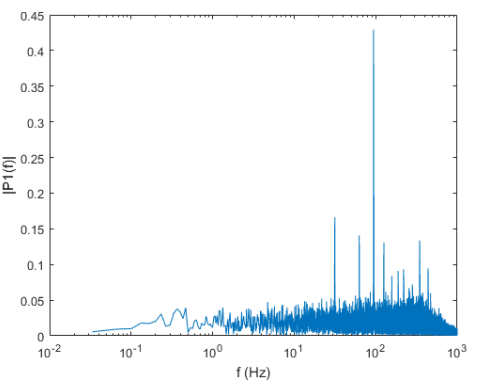

(e) $-(\mathrm{x} / \mathrm{d}=1.61, \mathrm{y} / \mathrm{d}=2.21)$

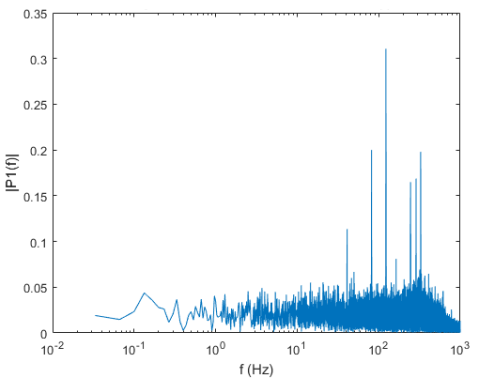

(f) $-(\mathrm{x} / \mathrm{d}=1.61, \mathrm{y} / \mathrm{d}=2.21)$

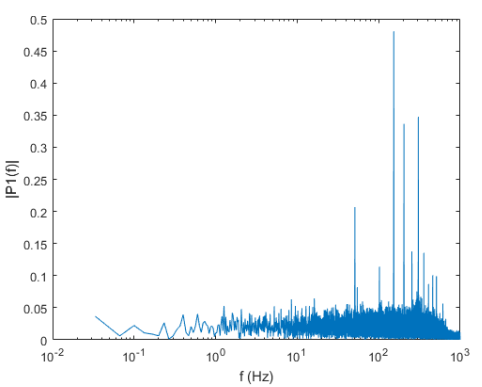

$(\mathrm{g})-(\mathrm{x} / \mathrm{d}=1.61, \mathrm{y} / \mathrm{d}=2.21)$

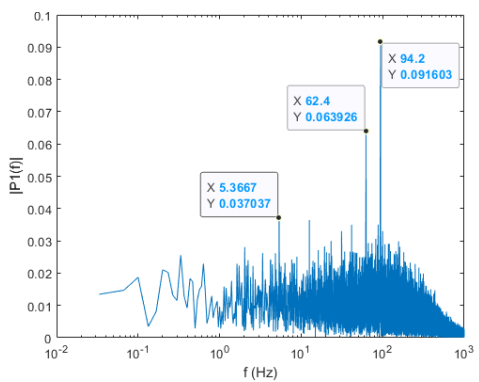

(e) $-(\mathrm{x} / \mathrm{d}=32.2, \mathrm{y} / \mathrm{d}=0)$

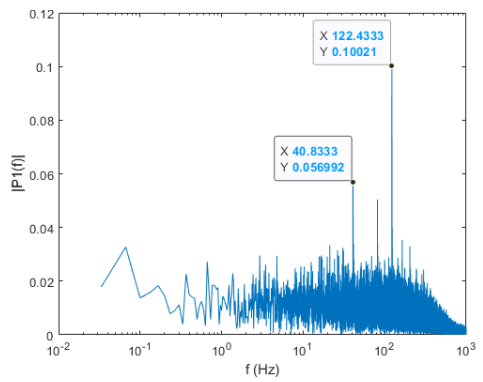

(f) $-(\mathrm{x} / \mathrm{d}=32.2, \mathrm{y} / \mathrm{d}=0)$

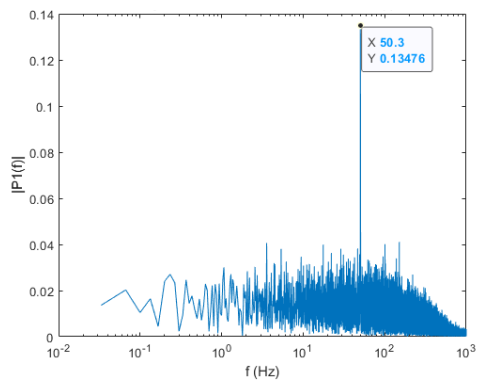

$(\mathrm{g})-(\mathrm{x} / \mathrm{d}=32.2, \mathrm{y} / \mathrm{d}=0)$

Figure B.4. Single sided frequency spectrum at Reynold Number 1250 and Rotation RPM (a) 120 (b) 360 (c) 960 (d) 1500 (e) 1800 (f) 2400 (g) 3000 RPM (Continued) 


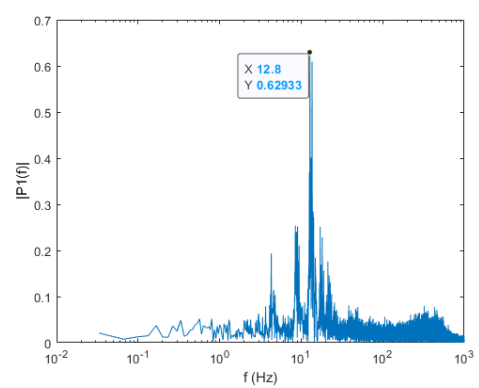

(a) $-(x / d=1.61, y / d=0)$

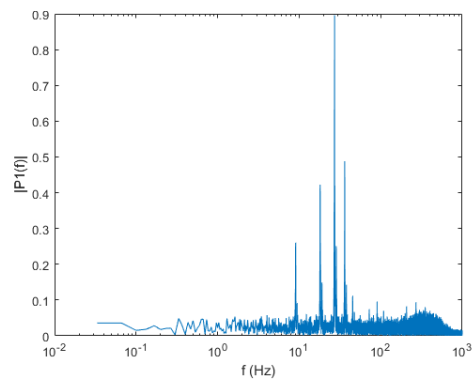

(b) $-(\mathrm{x} / \mathrm{d}=1.61, \mathrm{y} / \mathrm{d}=0)$

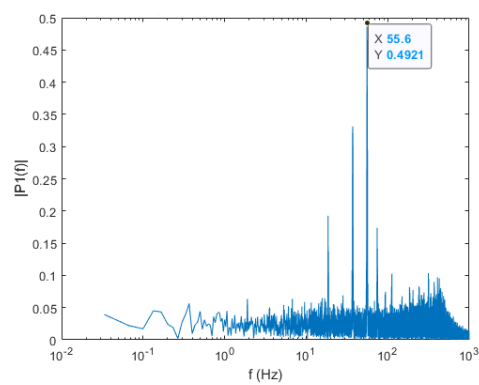

(c) $-(\mathrm{x} / \mathrm{d}=1.61, \mathrm{y} / \mathrm{d}=0)$

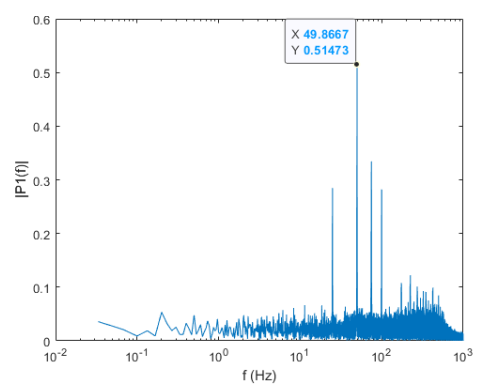

$(d)-(x / d=1.61, y / d=0)$

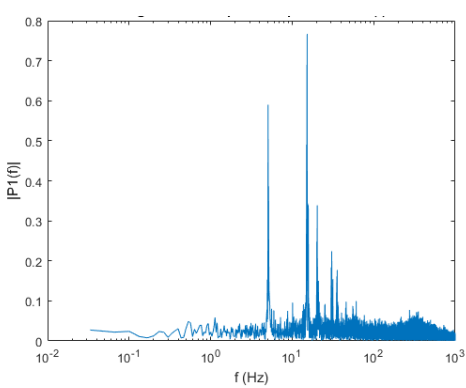

(a) $-(x / d=1.61, y / d=2.21)$

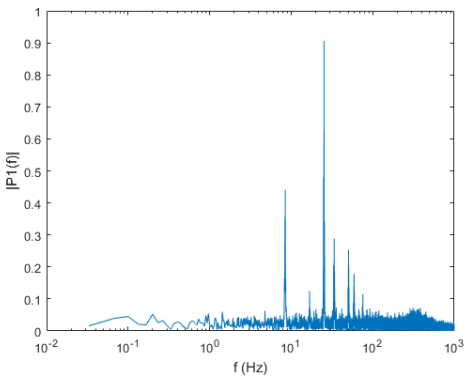

(b) $-(x / d=1.61, y / d=2.21)$

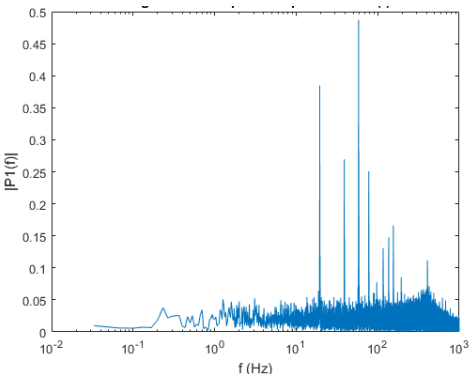

(c) $-(x / d=1.61, y / d=2.21)$

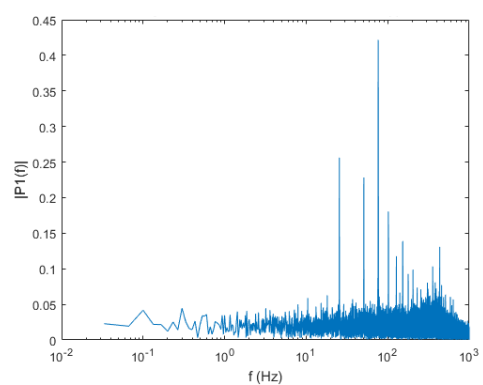

(d) $-(x / d=1.61, y / d=2.21)$

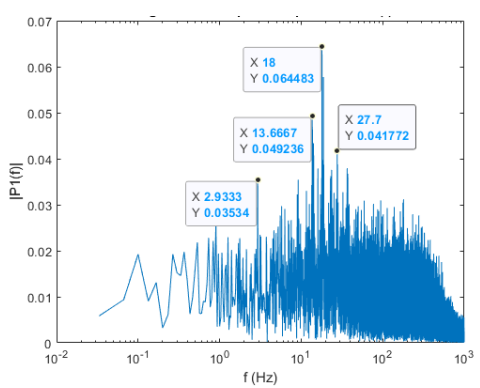

(a) $-(x / d=32.2, y / d=0)$

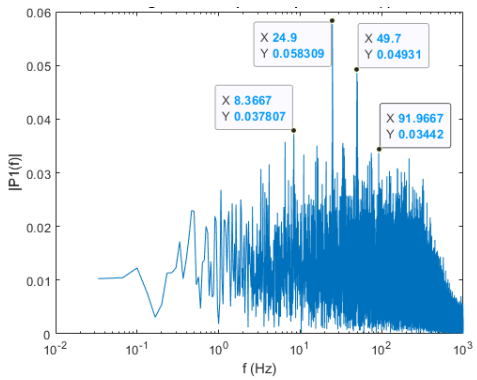

(b) $-(\mathrm{x} / \mathrm{d}=32.2, \mathrm{y} / \mathrm{d}=0)$

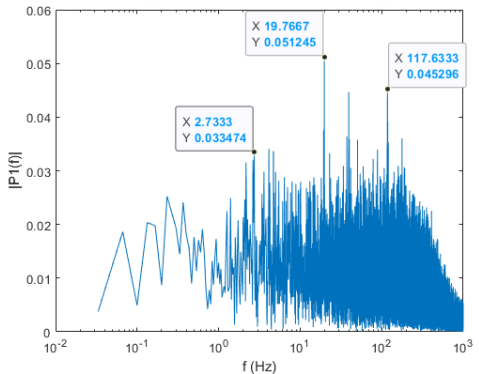

(c) $-(\mathrm{x} / \mathrm{d}=32.2, \mathrm{y} / \mathrm{d}=0)$

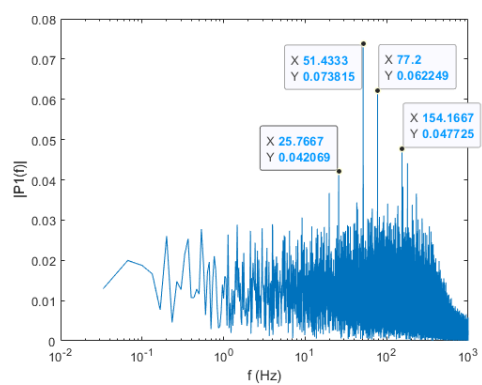

(d) $-(\mathrm{x} / \mathrm{d}=32.2, \mathrm{y} / \mathrm{d}=0)$

Figure B.5. Single sided frequency spectrum at Reynold Number 1700 and Rotation RPM (a) 120 (b) 360 (c) 960 (d) 1500 (e) 1800 (f) 2400 (g) 3000 RPM 


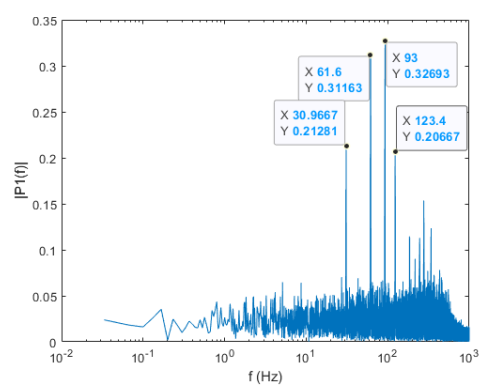

(e) $-(\mathrm{x} / \mathrm{d}=1.61, \mathrm{y} / \mathrm{d}=0)$

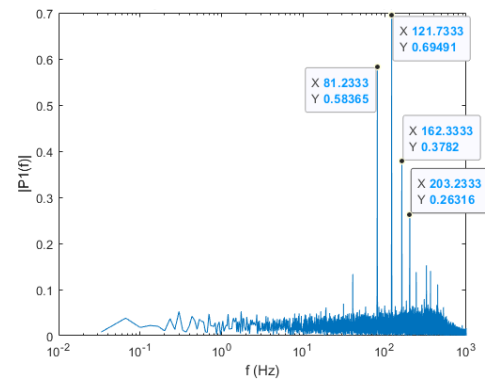

(f) $-(\mathrm{x} / \mathrm{d}=1.61, \mathrm{y} / \mathrm{d}=0)$

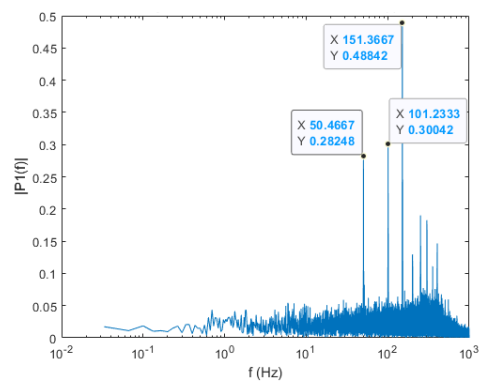

$(\mathrm{g})-(\mathrm{x} / \mathrm{d}=1.61, \mathrm{y} / \mathrm{d}=0)$

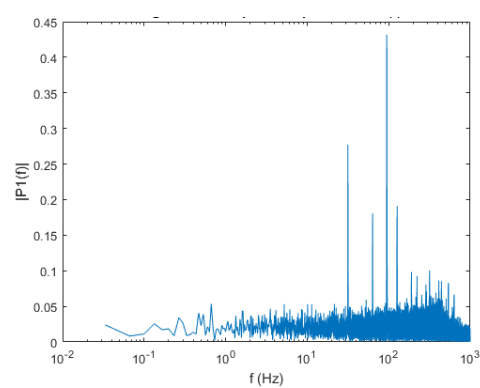

(e) $-(\mathrm{x} / \mathrm{d}=1.61, \mathrm{y} / \mathrm{d}=2.21)$

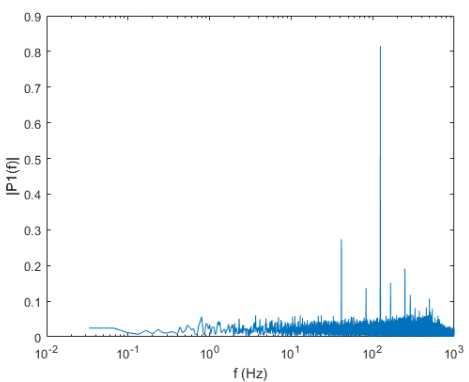

(f) $-(x / d=1.61, y / d=2.21)$

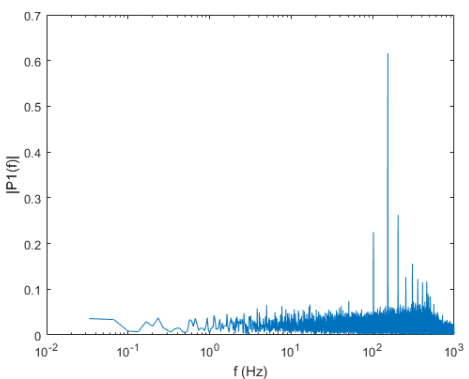

$(g)-(x / d=1.61, y / d=2.21)$

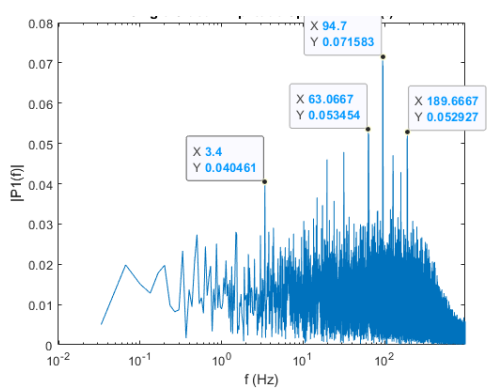

(e) $-(\mathrm{x} / \mathrm{d}=32.2, \mathrm{y} / \mathrm{d}=0)$

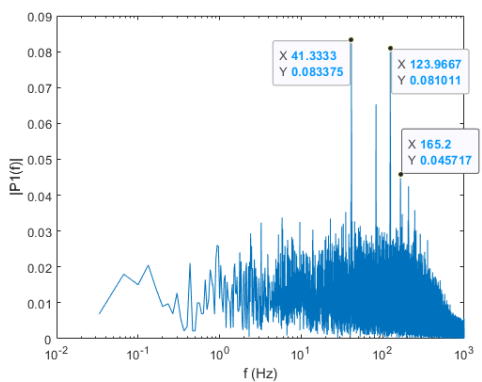

(f) $-(\mathrm{x} / \mathrm{d}=32.2, \mathrm{y} / \mathrm{d}=0)$

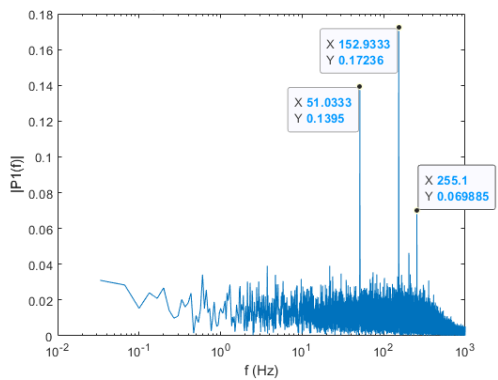

$(\mathrm{g})-(\mathrm{x} / \mathrm{d}=32.2, \mathrm{y} / \mathrm{d}=0)$

Figure B.5. Single sided frequency spectrum at Reynold Number 1700 and Rotation RPM (a) 120 (b) 360 (c) 960 (d) 1500 (e) 1800 (f) 2400 (g) 3000 RPM (Continued) 

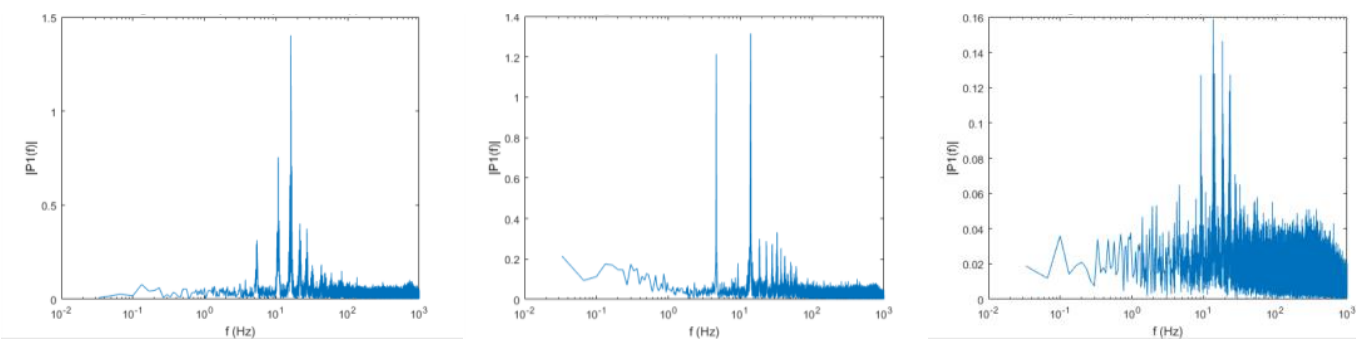

(a) $-(x / d=1.61, y / d=0)$

(a) $-(x / d=1.61, y / d=2.21)$

(a) $-(x / d=32.2, y / d=0)$
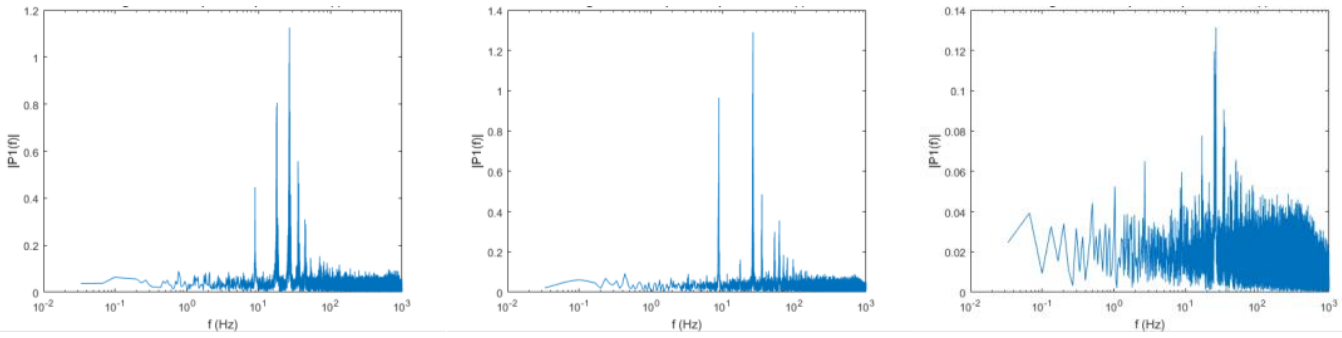

(b)-

$(\mathrm{x} / \mathrm{d}=1.61, \mathrm{y} / \mathrm{d}=0)$

(b) $-(x / d=1.61, y / d=2.21)$

(b) $-(\mathrm{x} / \mathrm{d}=32.2, \mathrm{y} / \mathrm{d}=0)$
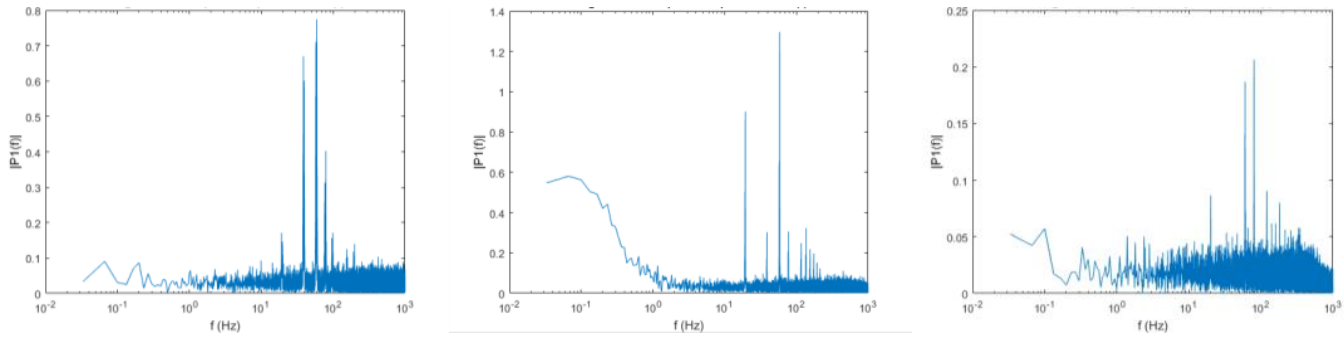

(c)-

$(\mathrm{x} / \mathrm{d}=1.61, \mathrm{y} / \mathrm{d}=0)$

(c) $-(x / d=1.61, y / d=2.21)$

(c) $-(\mathrm{x} / \mathrm{d}=32.2, \mathrm{y} / \mathrm{d}=0)$
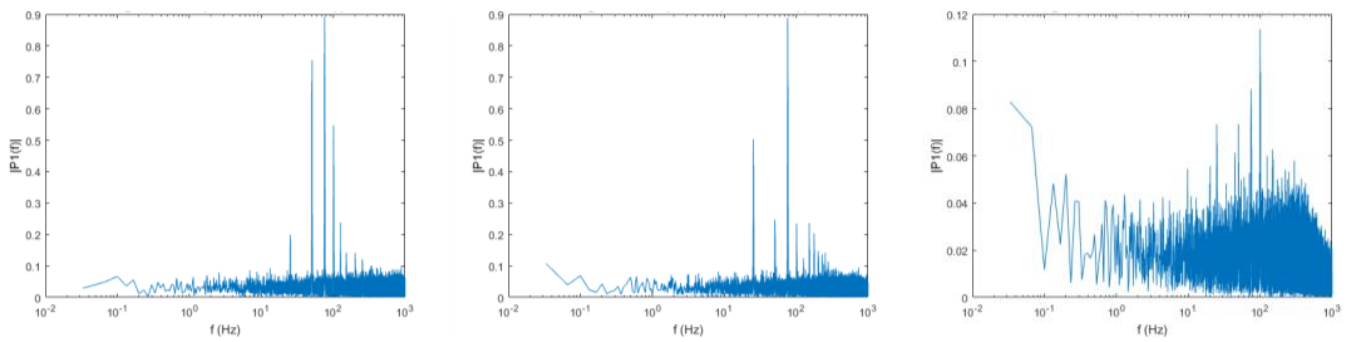

(d) $-(x / d=1.61, y / d=0)$

(d) $-(x / d=1.61, y / d=2.21)$

(d) $-(x / d=32.2, y / d=0)$

Figure B.6. Single sided frequency spectrum at Reynold Number 4350 and Rotation RPM (a) 120 (b) 360 (c) 960 (d) 1500 (e) 1800 (f) 2400 (g) 3000 RPM 


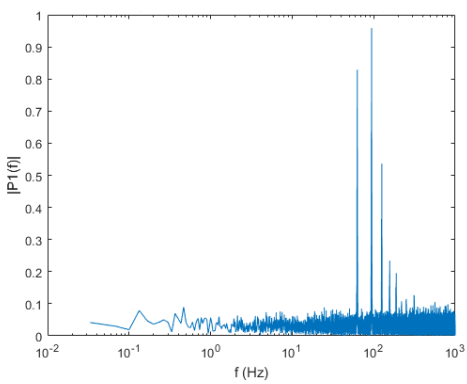

(e) $-(\mathrm{x} / \mathrm{d}=1.61, \mathrm{y} / \mathrm{d}=0)$

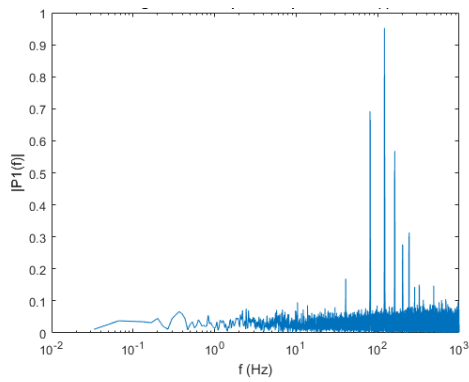

(f)-(x/d=1.61, y/d=0)

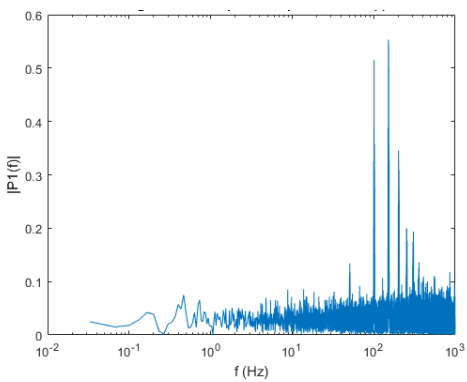

$(\mathrm{g})-(\mathrm{x} / \mathrm{d}=1.61, \mathrm{y} / \mathrm{d}=0)$

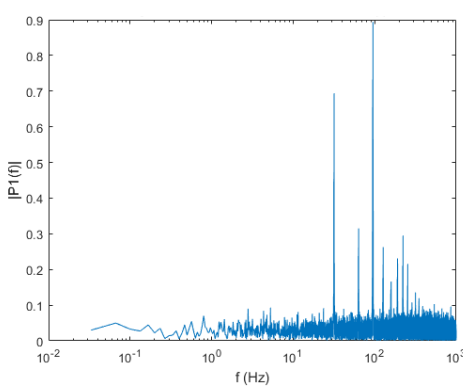

(e) $-(\mathrm{x} / \mathrm{d}=1.61, \mathrm{y} / \mathrm{d}=2.21)$

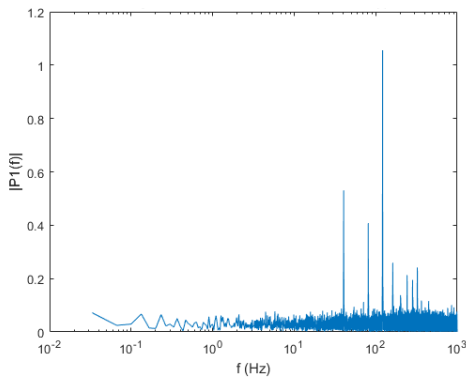

(f) $-(\mathrm{x} / \mathrm{d}=1.61, \mathrm{y} / \mathrm{d}=2.21)$

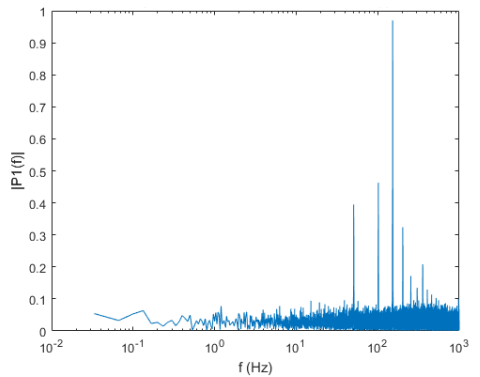

$(\mathrm{g})-(\mathrm{x} / \mathrm{d}=1.61, \mathrm{y} / \mathrm{d}=2.21)$

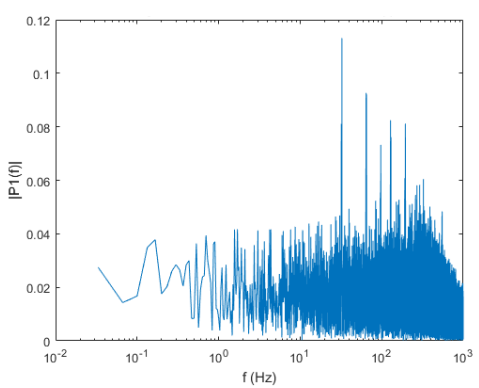

(e) $-(\mathrm{x} / \mathrm{d}=32.2, \mathrm{y} / \mathrm{d}=0)$

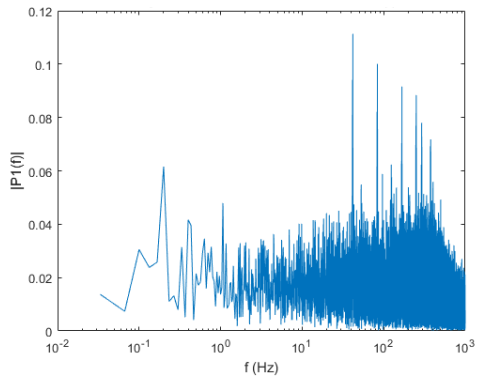

(f) $-(\mathrm{x} / \mathrm{d}=32.2, \mathrm{y} / \mathrm{d}=0)$

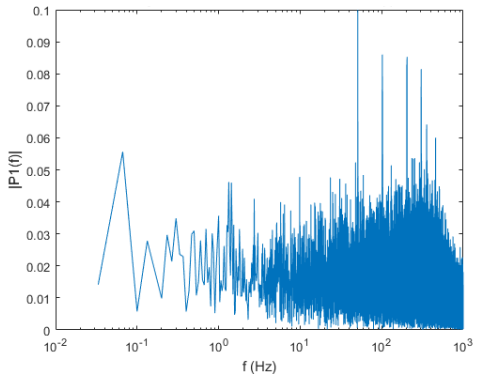

$(\mathrm{g})-(\mathrm{x} / \mathrm{d}=32.2, \mathrm{y} / \mathrm{d}=0)$

Figure B.6. Single sided frequency spectrum at Reynold Number 4350 and Rotation RPM (a) 120 (b) 360 (c) 960 (d) 1500 (e) 1800 (f) 2400 (g) 3000 RPM (Continued) 


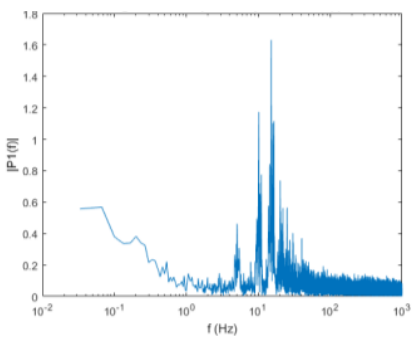

(a) $-(x / d=1.61, y / d=0)$

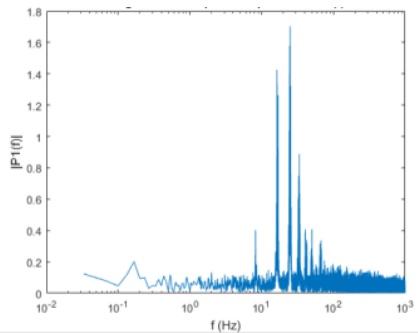

(b) $-(\mathrm{x} / \mathrm{d}=1.61, \mathrm{y} / \mathrm{d}=0)$

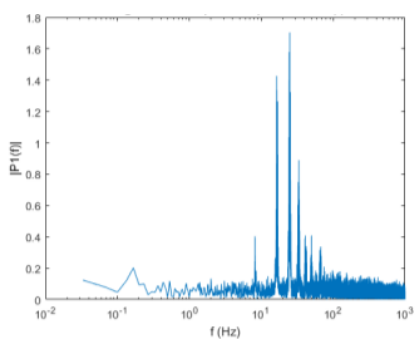

(c) $-(\mathrm{x} / \mathrm{d}=1.61, \mathrm{y} / \mathrm{d}=0)$

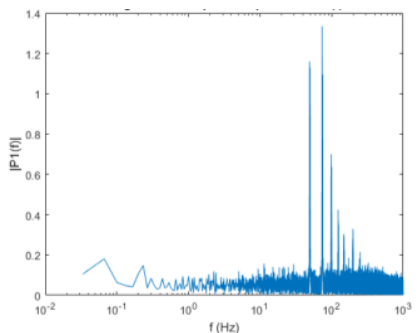

(d) $-(\mathrm{x} / \mathrm{d}=1.61, \mathrm{y} / \mathrm{d}=0)$

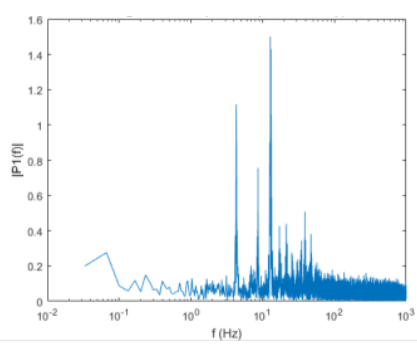

(a) $-(\mathrm{x} / \mathrm{d}=1.61, \mathrm{y} / \mathrm{d}=2.21)$

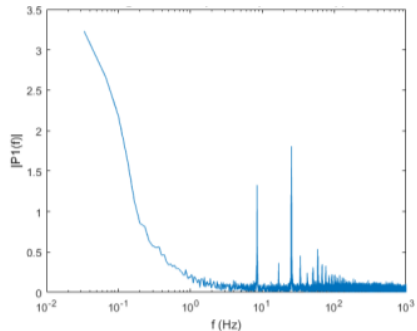

(b) $-(x / d=1.61, y / d=2.21)$

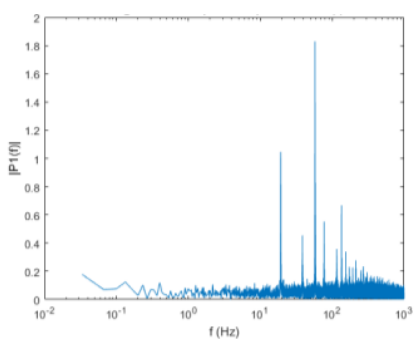

(c) $-(\mathrm{x} / \mathrm{d}=1.61, \mathrm{y} / \mathrm{d}=2.21)$

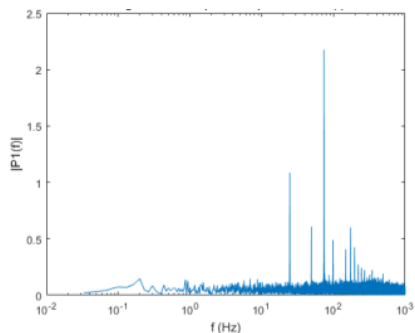

(d) $-(\mathrm{x} / \mathrm{d}=1.61, \mathrm{y} / \mathrm{d}=2.21)$

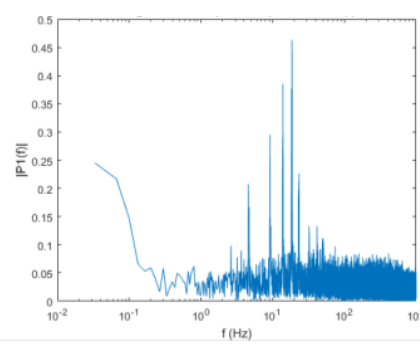

(a) $-(\mathrm{x} / \mathrm{d}=32.2, \mathrm{y} / \mathrm{d}=0)$

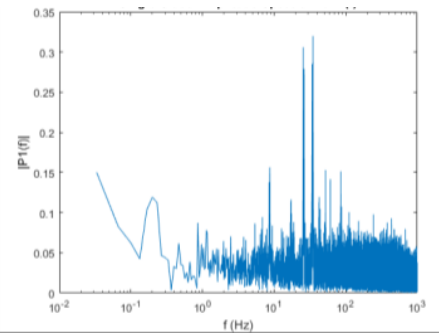

(b) $-(x / d=32.2, y / d=0)$

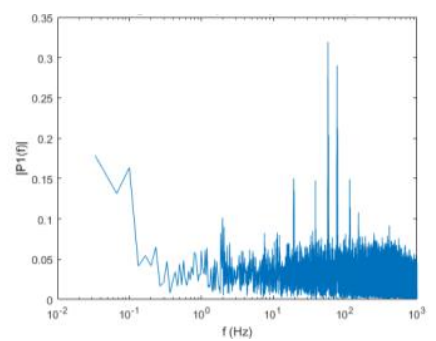

(c) $-(\mathrm{x} / \mathrm{d}=32.2, \mathrm{y} / \mathrm{d}=0)$

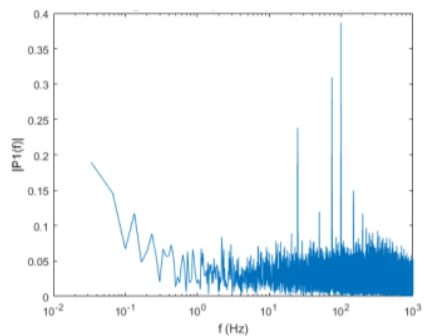

(d) $-(\mathrm{x} / \mathrm{d}=32.2, \mathrm{y} / \mathrm{d}=0)$

Figure B.7. Single sided frequency spectrum at Reynold Number 7470 and Rotation RPM (a) 120 (b) 360 (c) 960 (d) 1500 (e) 1800 (f) 2400 (g) 3000 RPM 


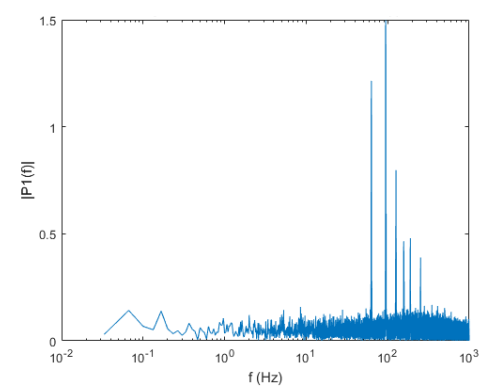

(e) $-(\mathrm{x} / \mathrm{d}=1.61, \mathrm{y} / \mathrm{d}=0)$

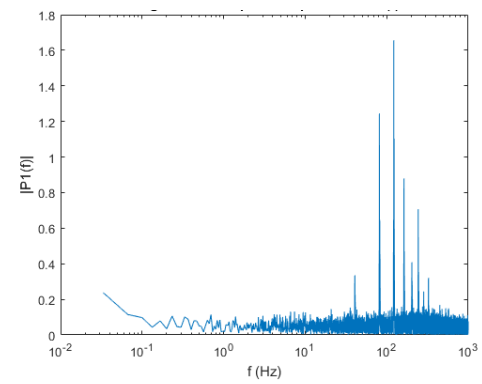

(f) $-(\mathrm{x} / \mathrm{d}=1.61, \mathrm{y} / \mathrm{d}=0)$

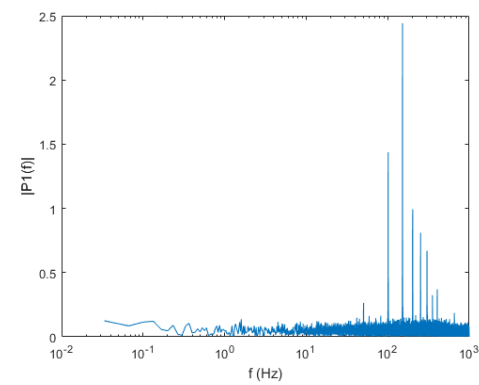

(g) $-(\mathrm{x} / \mathrm{d}=1.61, \mathrm{y} / \mathrm{d}=0)$

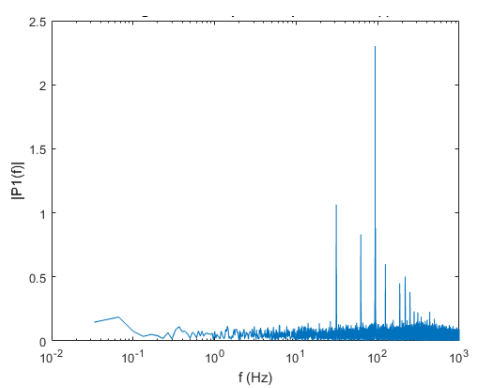

(e) $-(\mathrm{x} / \mathrm{d}=1.61, \mathrm{y} / \mathrm{d}=2.21)$

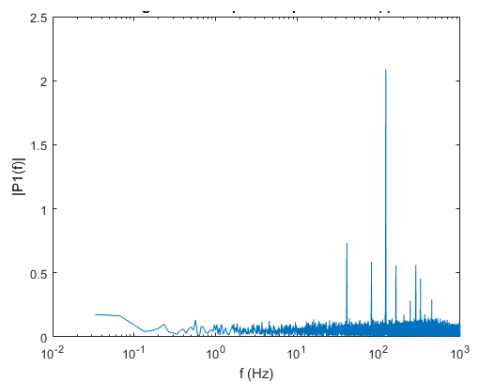

(f) $-(\mathrm{x} / \mathrm{d}=1.61, \mathrm{y} / \mathrm{d}=2.21)$

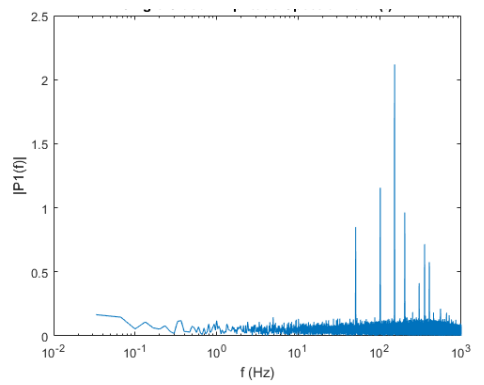

(g) $-(x / d=1.61, y / d=2.21)$

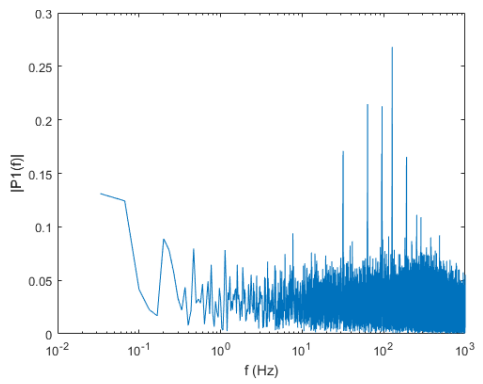

(e) $-(\mathrm{x} / \mathrm{d}=32.2, \mathrm{y} / \mathrm{d}=0)$

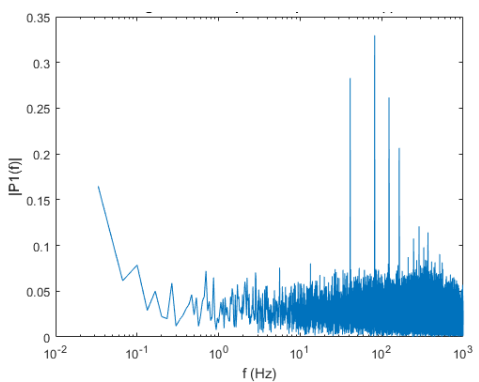

(f) $-(\mathrm{x} / \mathrm{d}=32.2, \mathrm{y} / \mathrm{d}=0)$

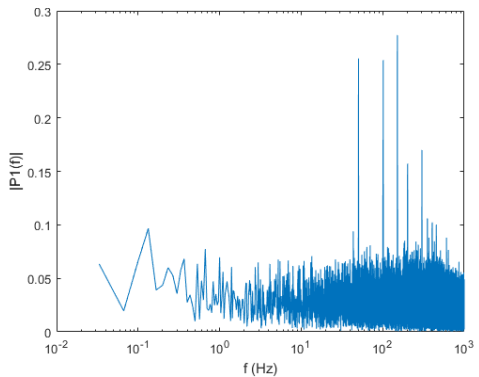

$(\mathrm{g})-(\mathrm{x} / \mathrm{d}=32.2, \mathrm{y} / \mathrm{d}=0)$

Figure B.7. Single sided frequency spectrum at Reynold Number 7470 and Rotation RPM (a) 120 (b) 360 (c) 960 (d) 1500 (e) 1800 (f) 2400 (g) 3000 RPM (Continued) 


\section{APPENDIX C}

\section{Uncertainty Calculation-Hotwire}

\section{Error Source}

Calibration measurement errors

Calibration equation errors

Calibration drift errors

Approximation errors

High frequency errors

Spatial resolution error

Disturbance error

Total Uncertainty

\section{Uncertainty}

$2 \%$

$0.1 \%$

$1.5 \%$

$-$

$2.5 \%$

\section{Uncertainty Calculation-PIV}

Error Source

Magnification Error

Timing Error

Image Displacement

Seeding Density error

Out of plane motion

Interrogation window size

Total Uncertainty

\section{Uncertainty}

$0.3 \%$

$0.01 \%$

$1.23 \%$

$1.2 \%$ 


\section{APPENDIX D}

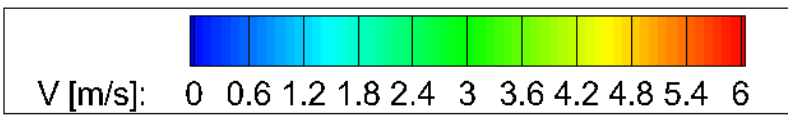

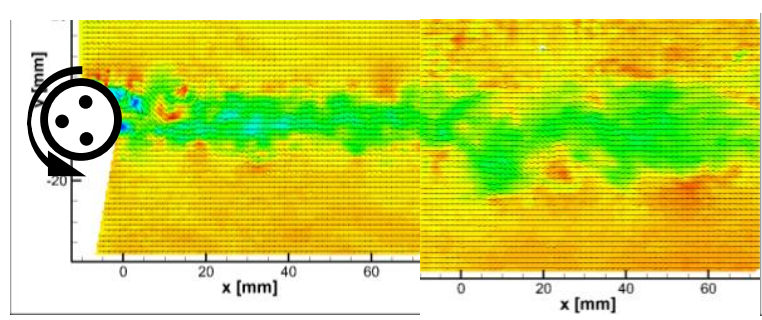

(a)

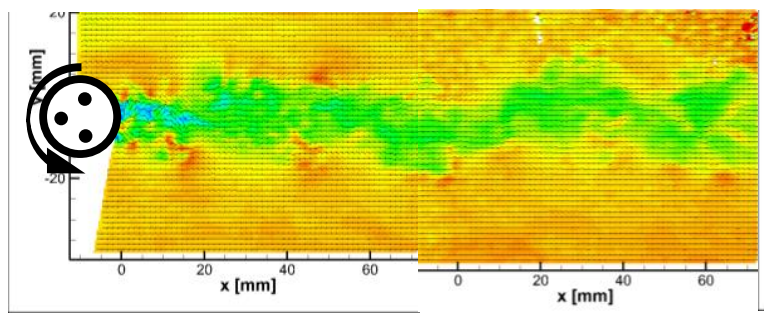

(b)

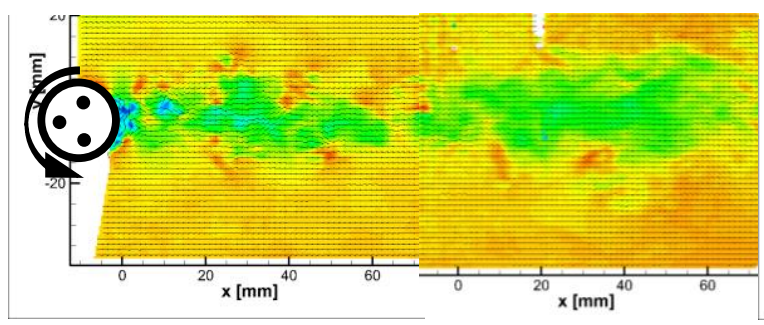

(e)

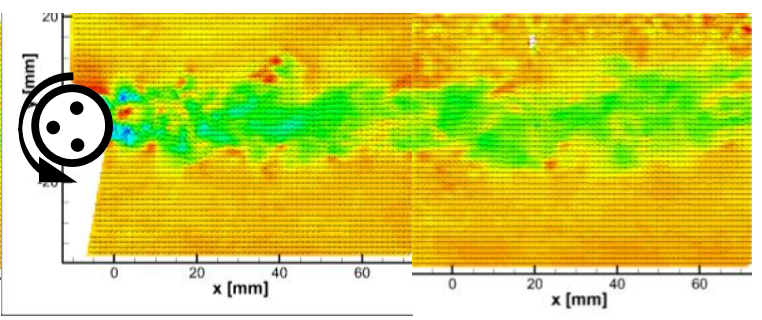

(b)

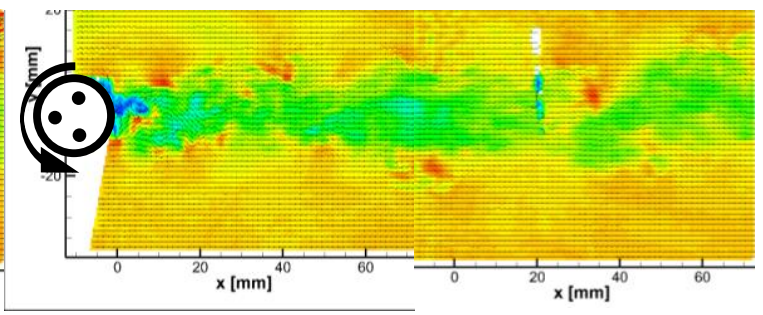

(d)

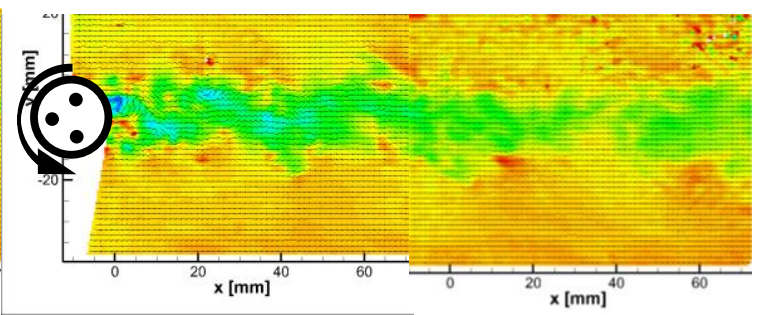

(f)

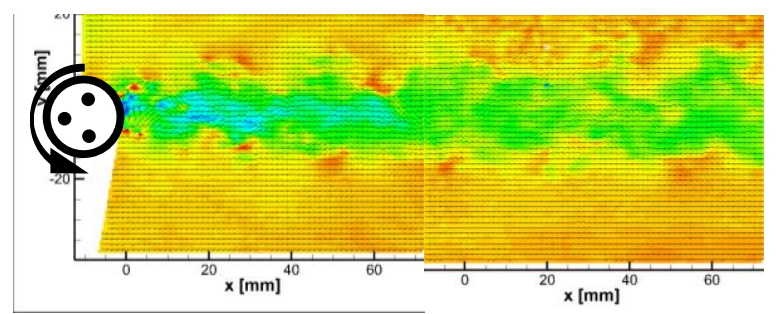

$(\mathrm{g})$

Figure D.1. Instantaneous Velocity Contour at $\mathrm{Re}=858$ and Rotation Speed is (a) 120 (b) 360 (c) 960 (d) 1500 (e) 1800 (f) 2400 (g) 3000 RPM 


\section{APPENDIX E}

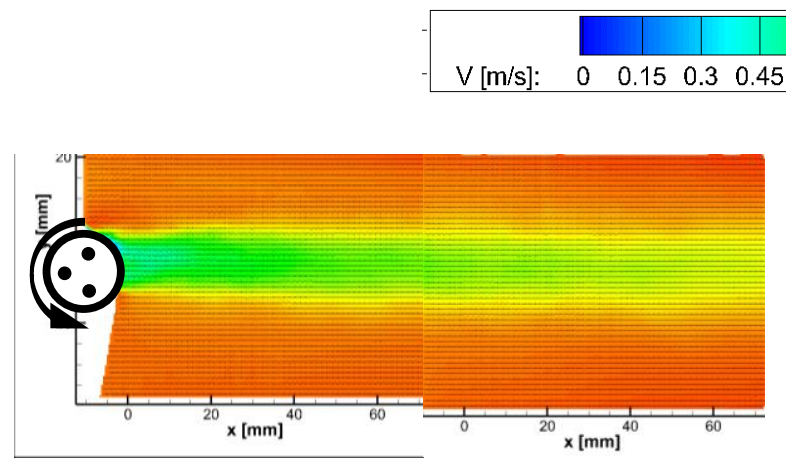

(a)

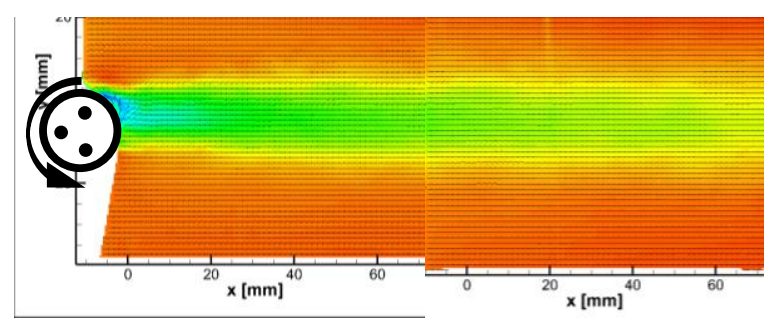

(c)

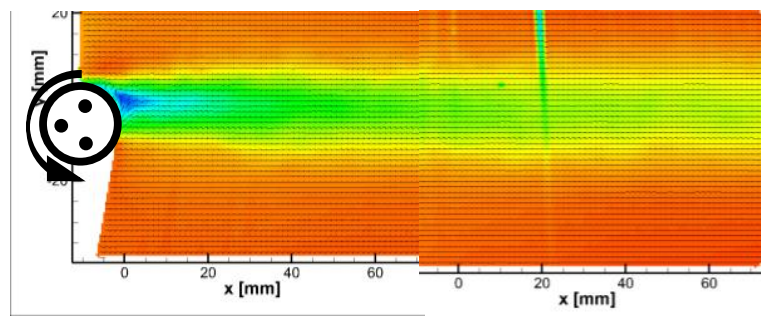

(e)

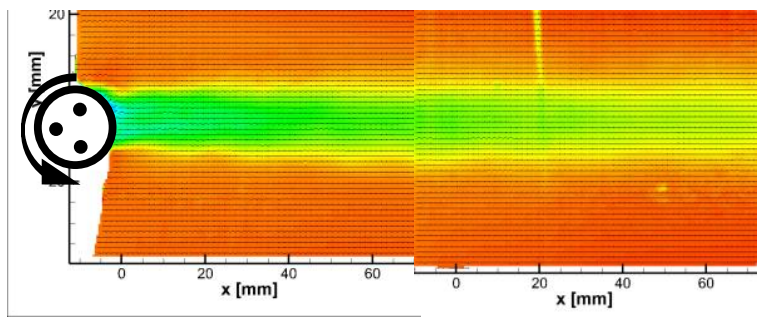

(b)

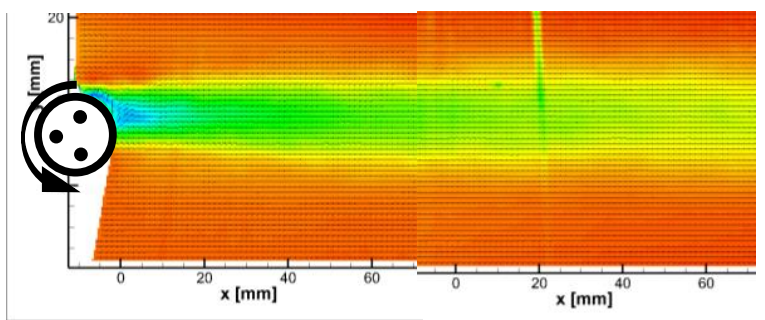

(d)

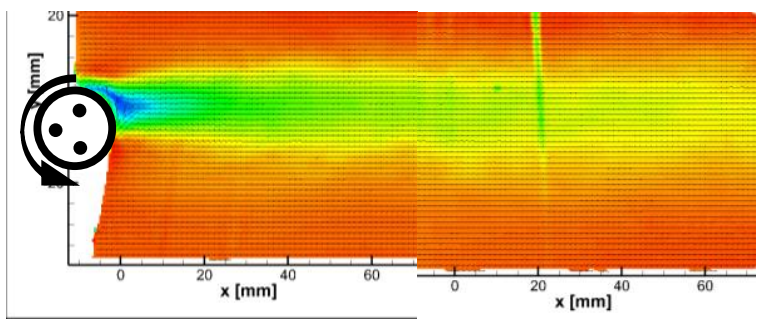

(f)

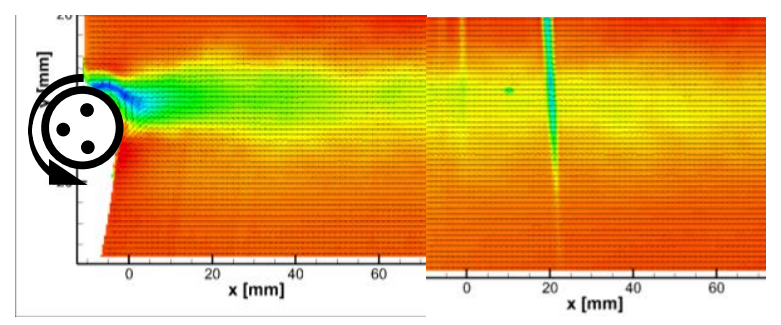

(g)

Figure E.1. Average Velocity Contour at Re=57 and Rotation Speed is (a) 120 (b) 360 (c) 960 (d) 1500 (e) 1800 (f) 2400 (g) 3000 RPM 


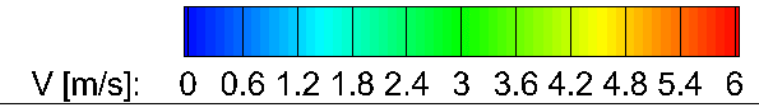

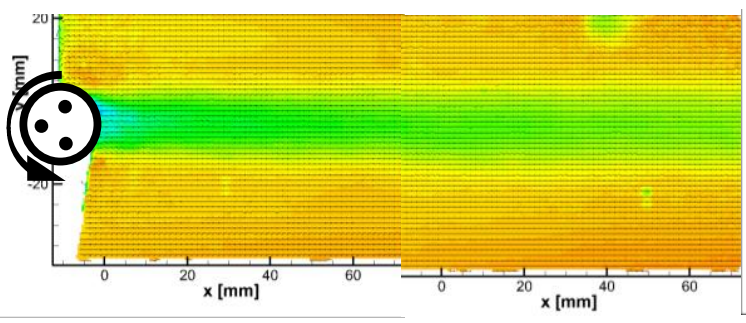

(a)

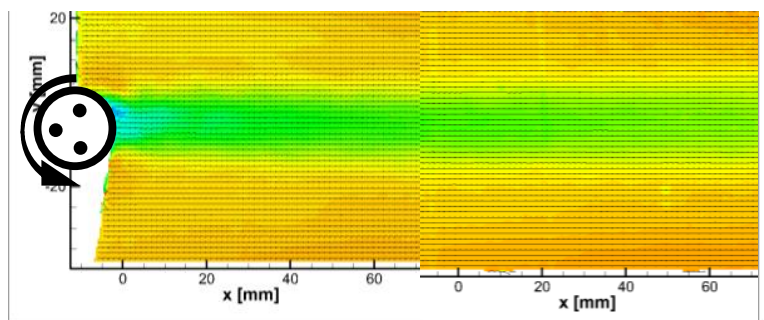

(b)

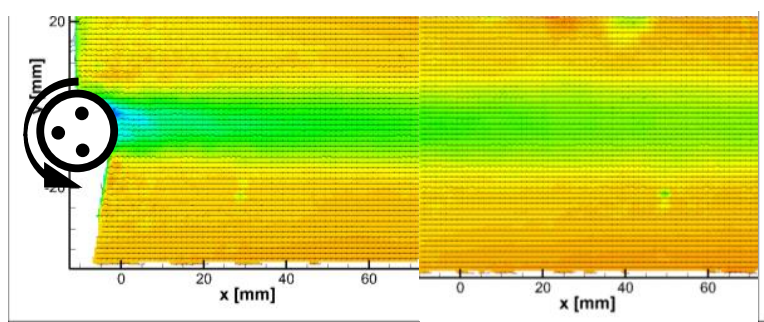

(e)

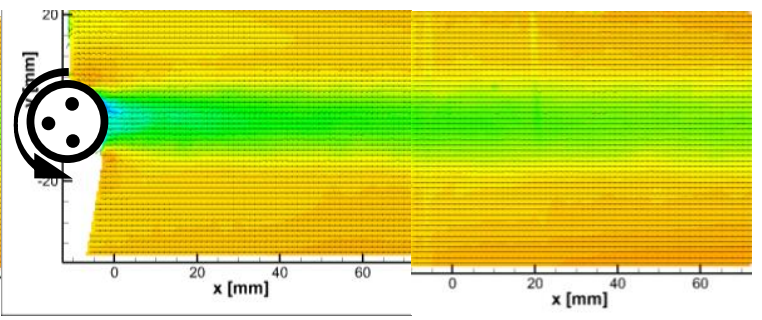

(b)

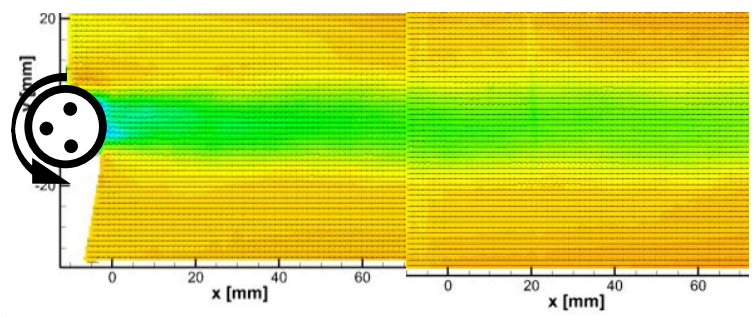

(d)

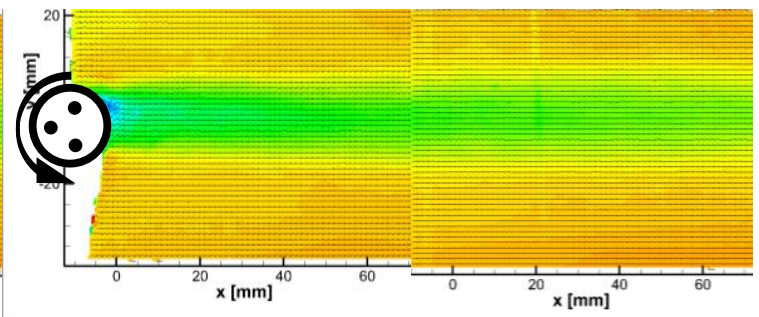

(f)

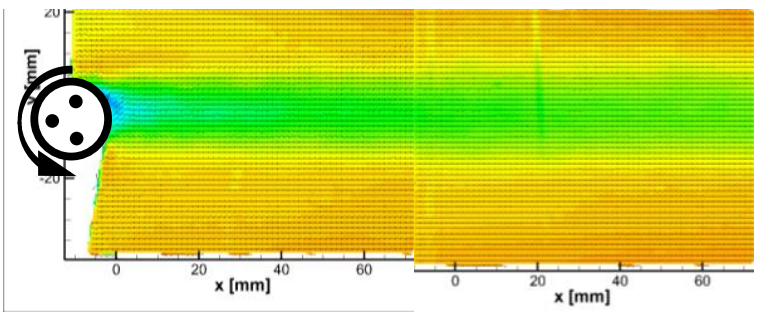

(g)

Figure E.2. Average Velocity Contour at Re=858 and Rotation Speed is (a) 120 (b) 360 (c) 960 (d) 1500 (e) 1800 (f) 2400 (g) 3000 RPM 


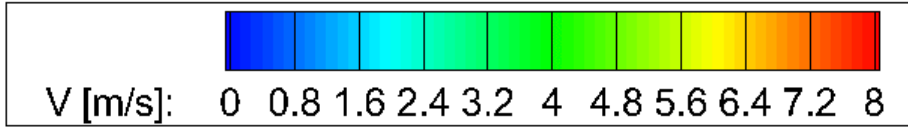

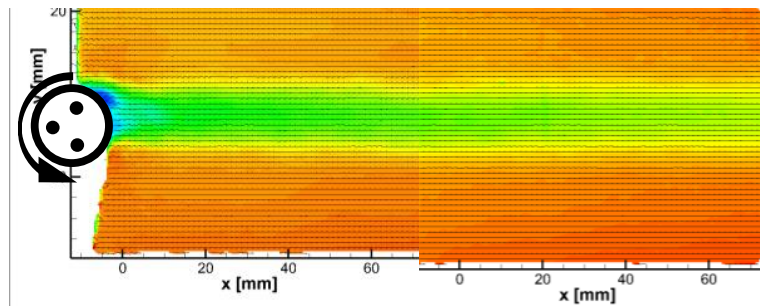

(a)

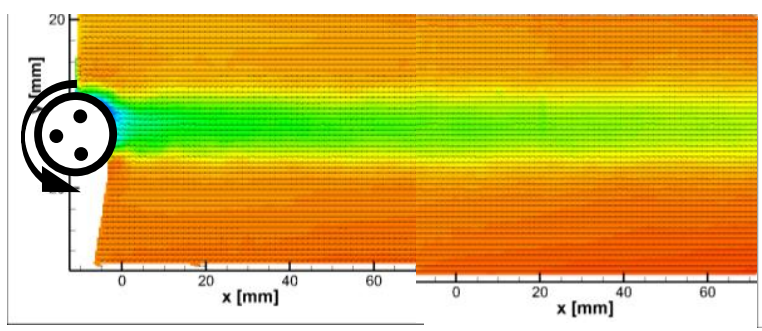

(c)

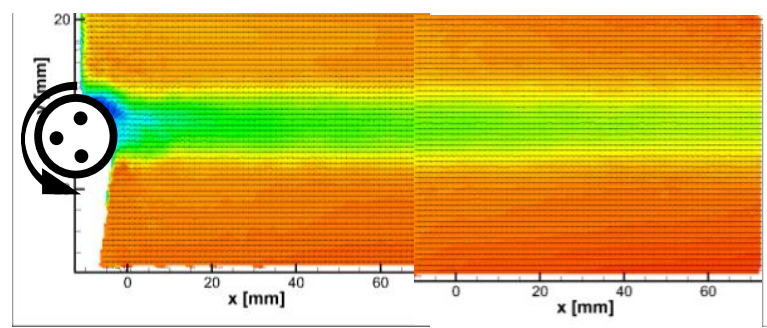

(e)

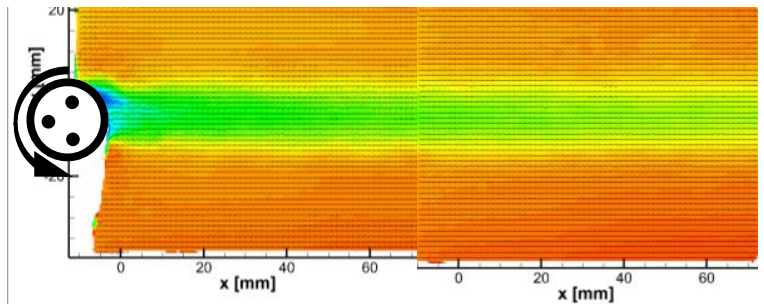

(b)

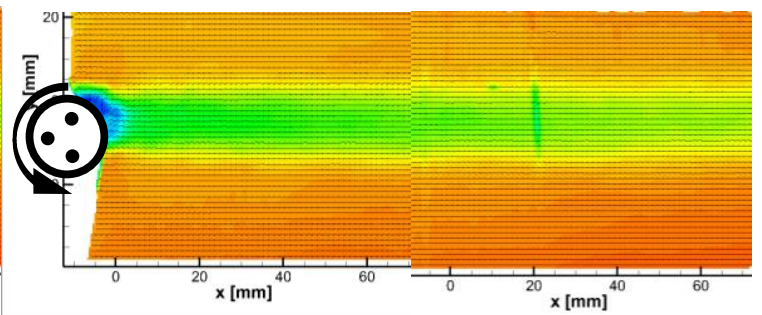

(d)

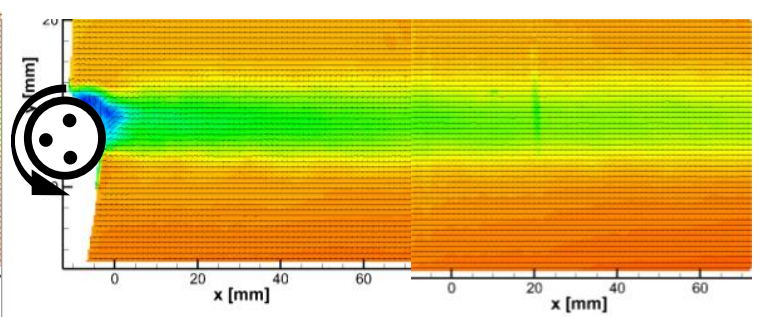

(f)

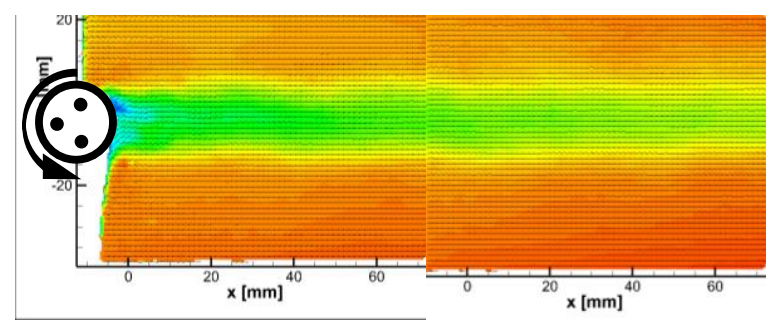

(g)

Figure E.3. Average Velocity Contour at $\mathrm{Re}=1250$ and Rotation Speed is (a) 120 (b) 360 (c) 960 (d) 1500 (e) 1800 (f) 2400 (g) 3000 RPM 


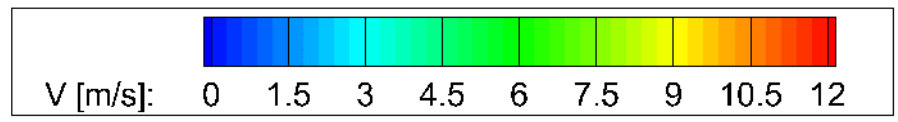

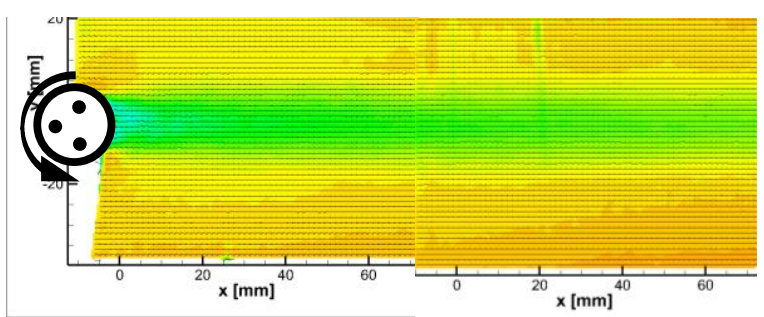

(a)

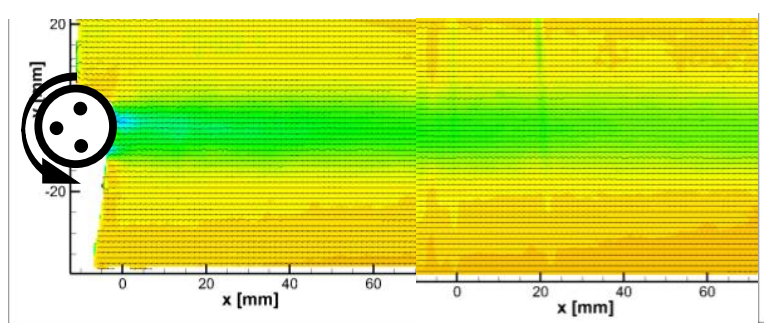

(c)

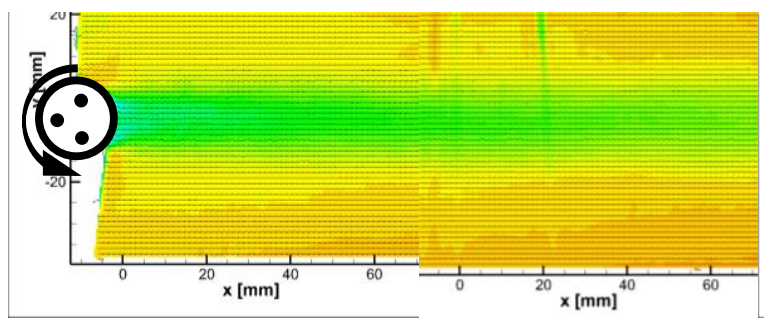

(e)

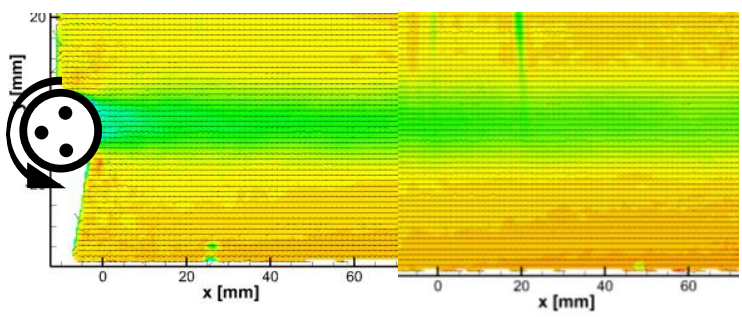

(b)

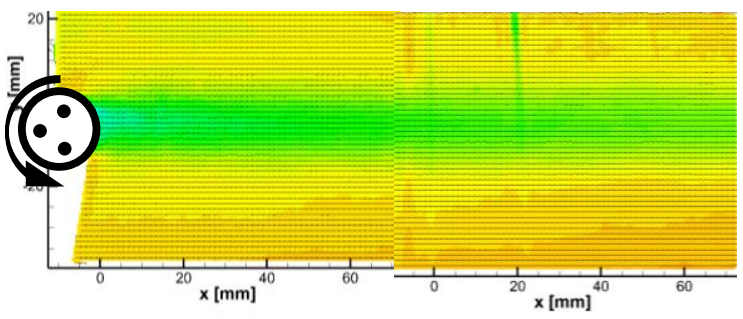

(d)

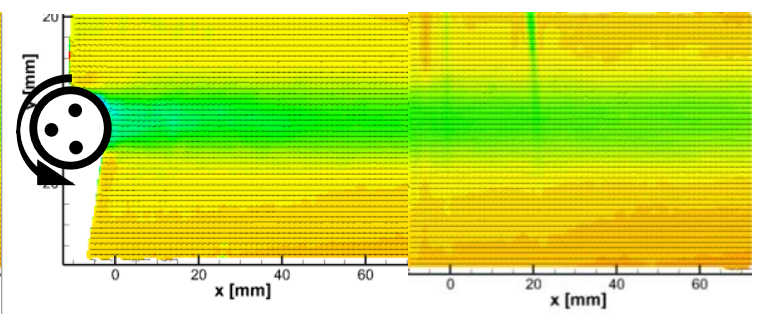

(f)

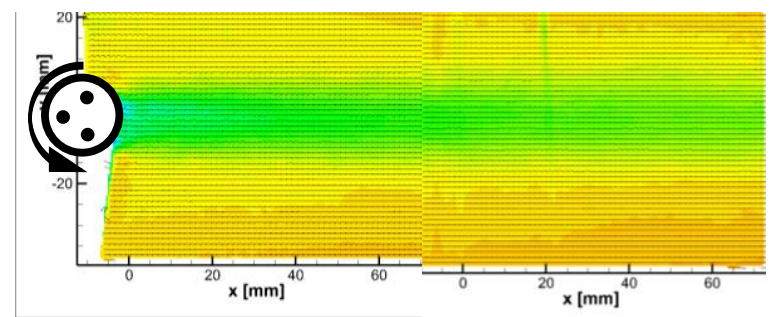

(g)

Figure E.4. Average Velocity Contour at $\mathrm{Re}=1700$ and Rotation Speed is (a) 120 (b) 360 (c) 960 (d) 1500 (e) 1800 (f) 2400 (g) 3000 RPM 


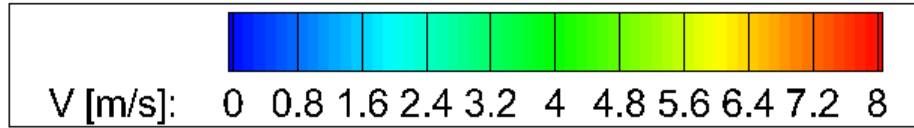

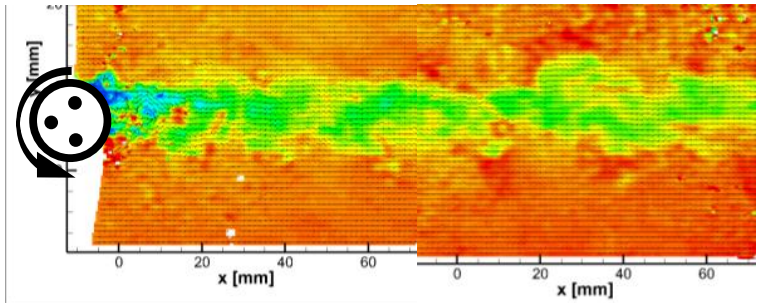

(a)

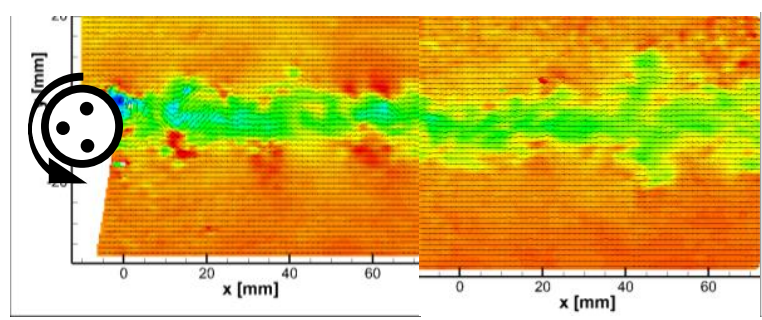

(c)

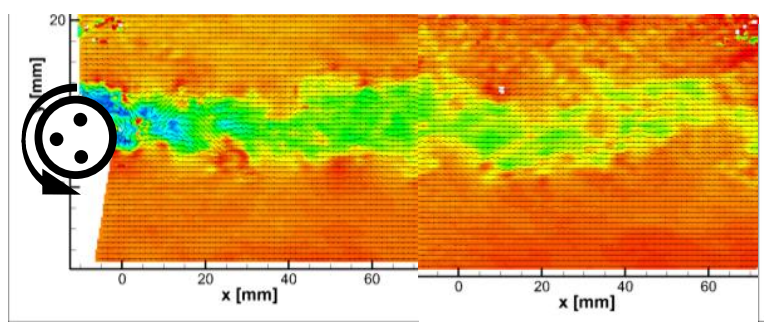

(e)

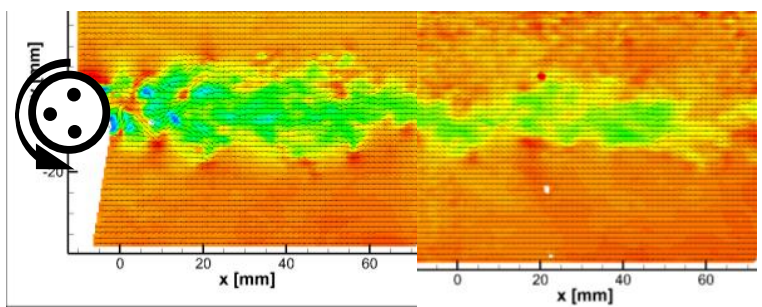

(b)

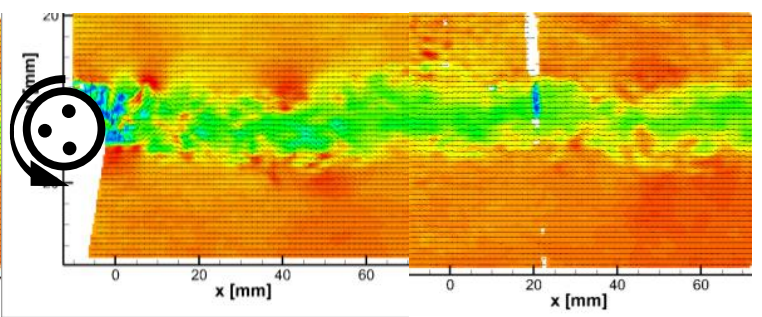

(d)

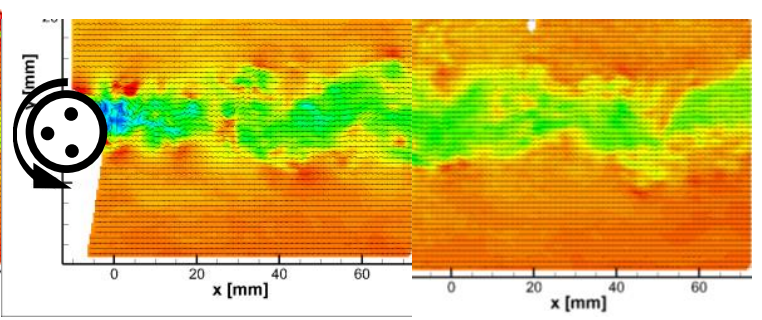

(f)

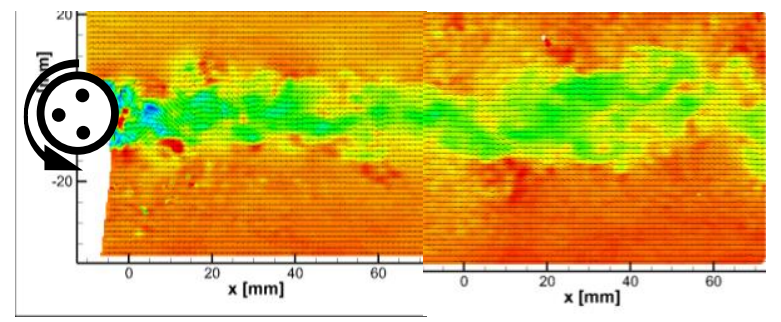

(g)

Figure E.5. Instantaneous Velocity Contour at $\mathrm{Re}=1250$ and Rotation Speed is (a) 120 (b) 360 (c) 960 (d) 1500 (e) 1800 (f) 2400 (g) 3000 RPM 


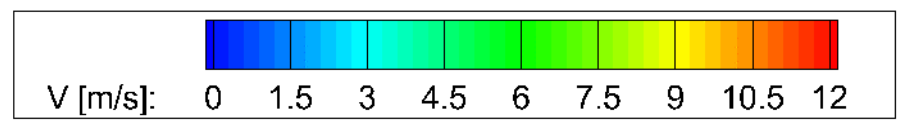

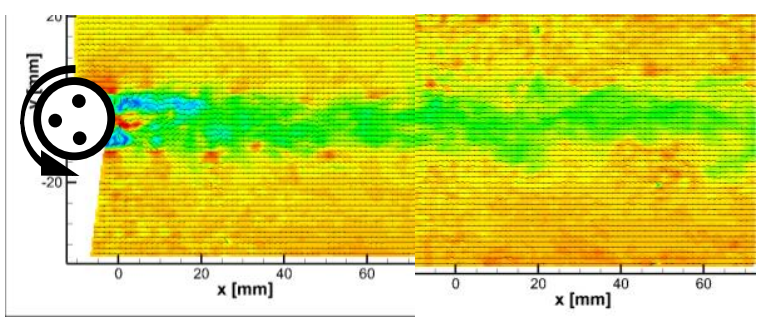

(a)

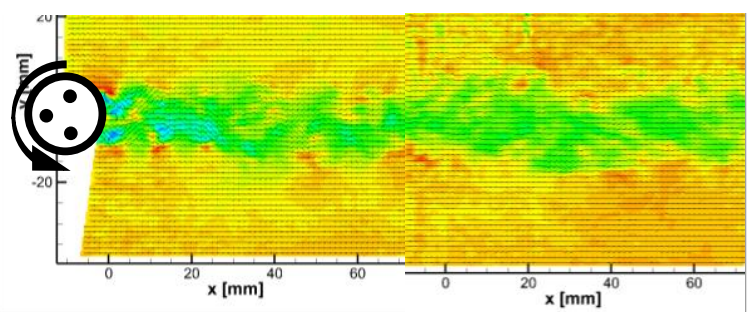

(c)

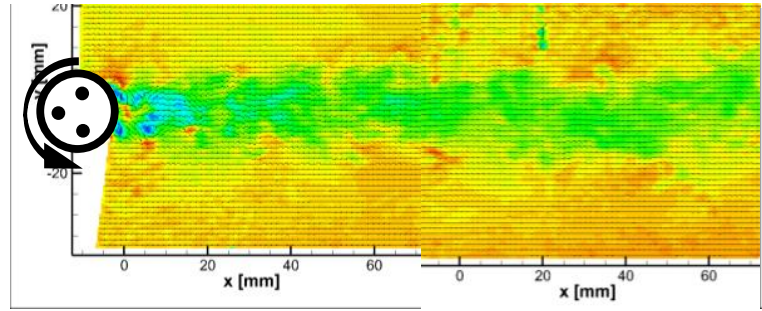

(e)

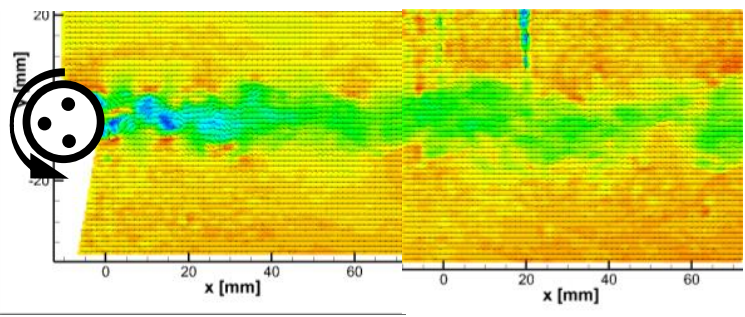

(b)

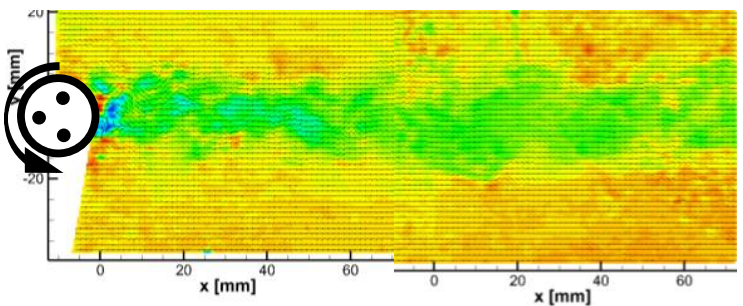

(d)

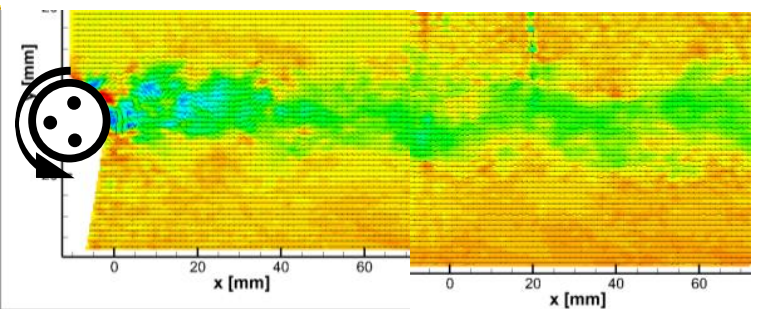

(f)

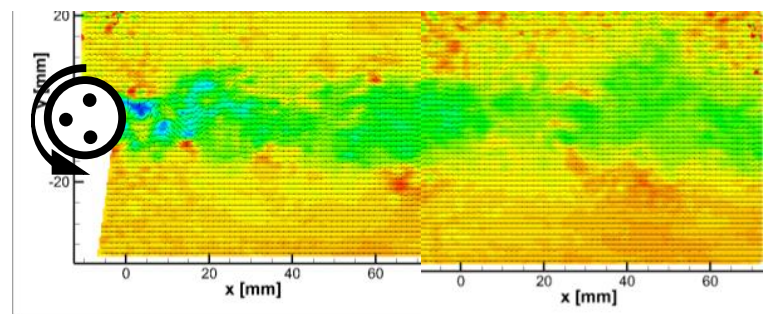

(g)

Figure E.6. Instantaneous Velocity Contour at $\mathrm{Re}=1700$ and Rotation Speed is (a) 120 (b) 360 (c) 960 (d) 1500 (e) 1800 (f) 2400 (g) 3000 RPM 


\section{APPENDIX F}

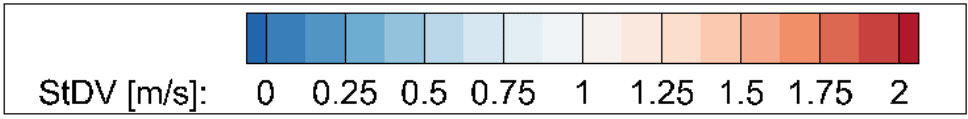

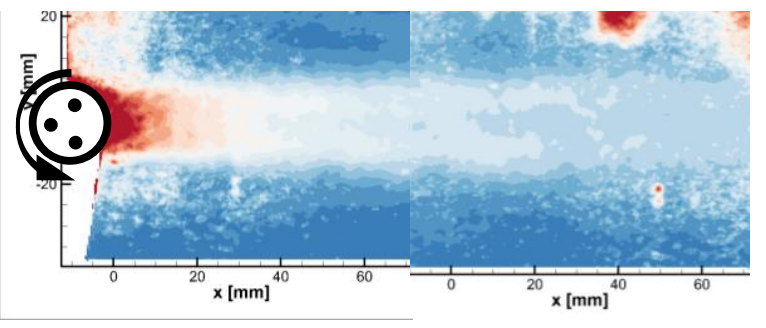

(a)

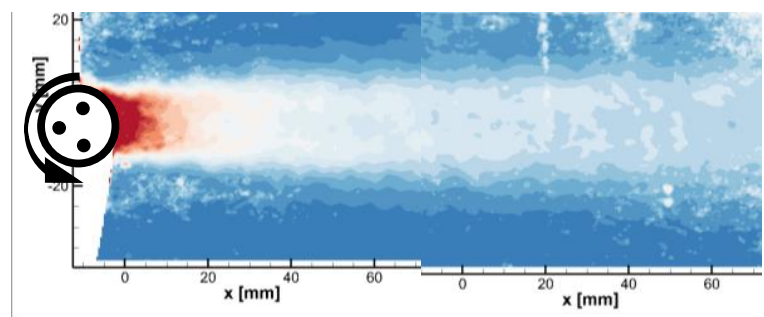

(c)

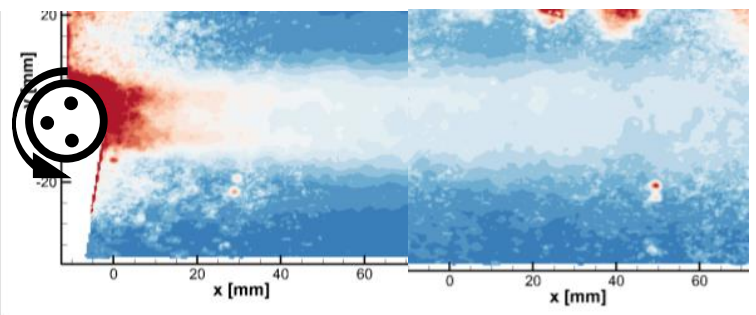

(e)

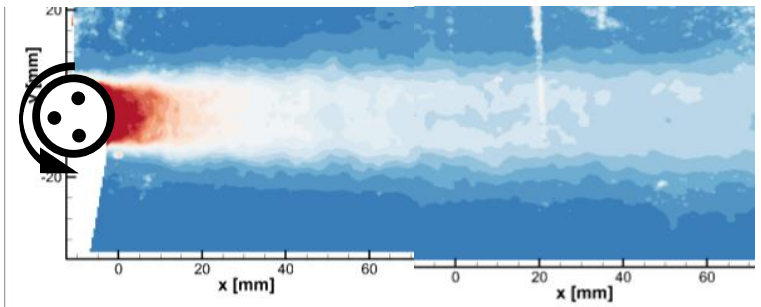

(b)

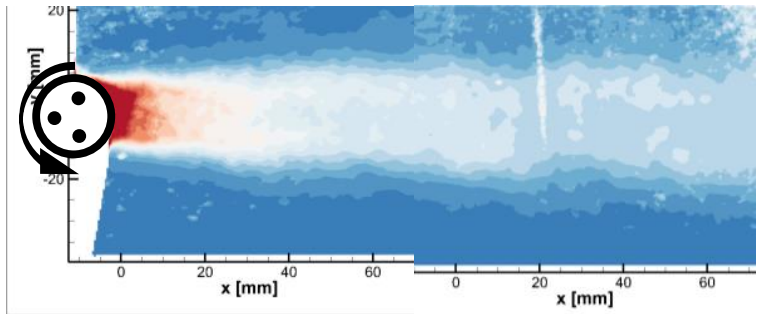

(d)

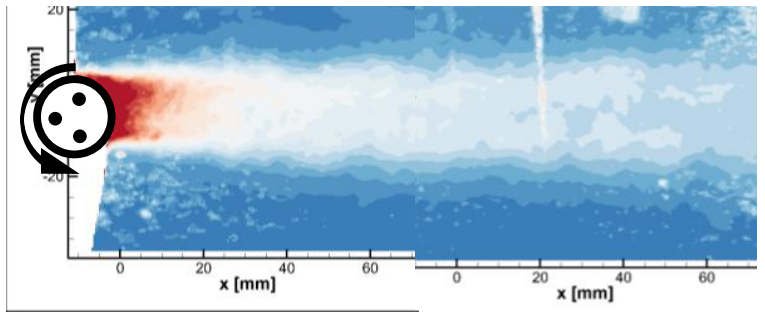

(f)

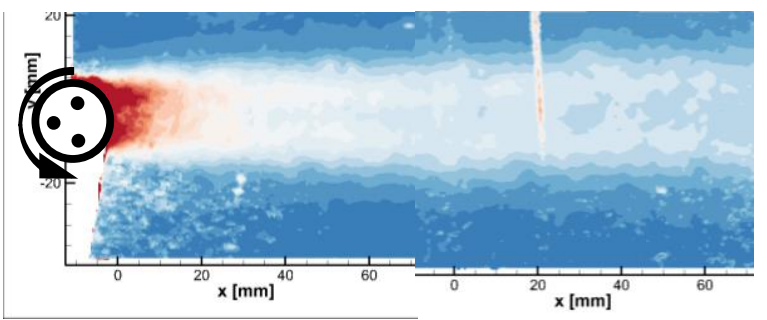

(g)

Figure F.1. Standard deviation at $\mathrm{Re}=858$ and Rotation Speed is (a) 120 (b) 360 (c) 960 (d) 1500 (e) 1800 (f) 2400 (g) 3000 RPM 


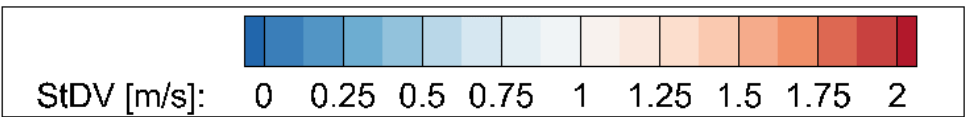

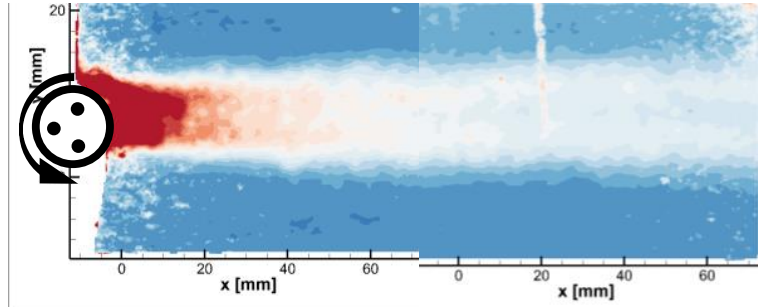

(a)

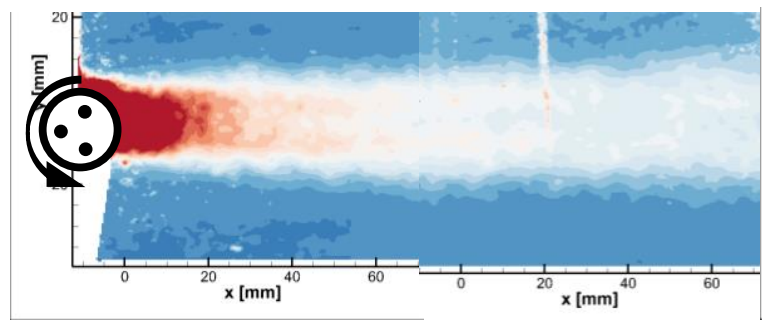

(c)

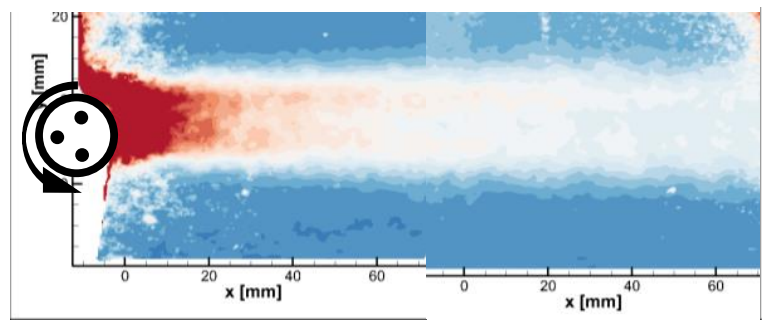

(e)

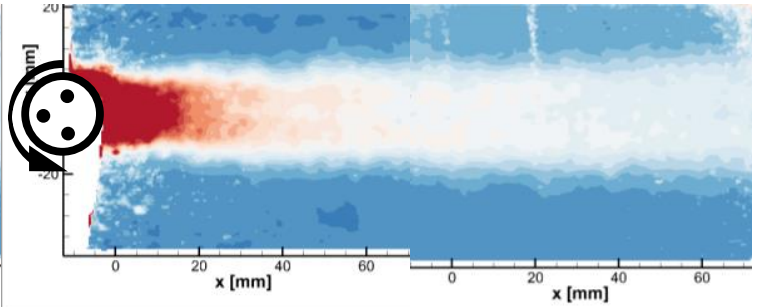

(b)

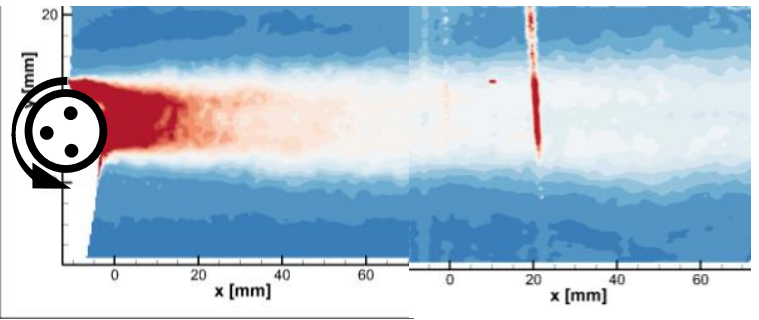

(d)

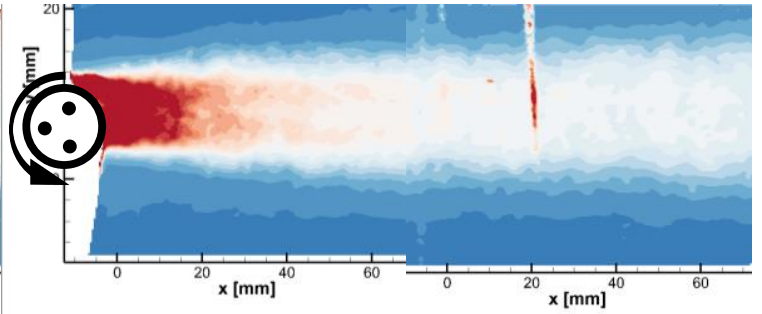

(f)

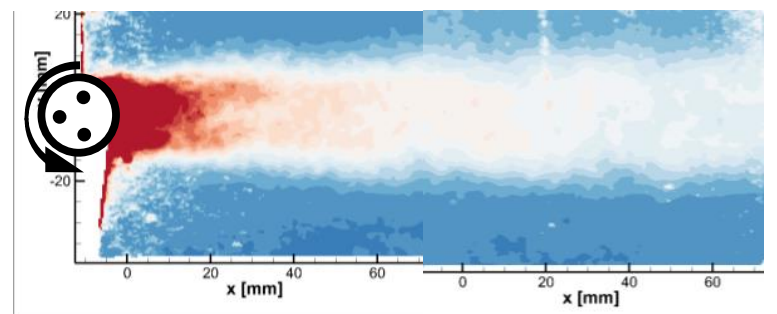

$(\mathrm{g})$

Figure F.2. Standard deviation at $R e=1250$ and Rotation Speed is (a) 120 (b) 360 (c) 960 (d) 1500 (e) 1800 (f) 2400 (g) 3000 RPM 


\section{APPENDIX G}

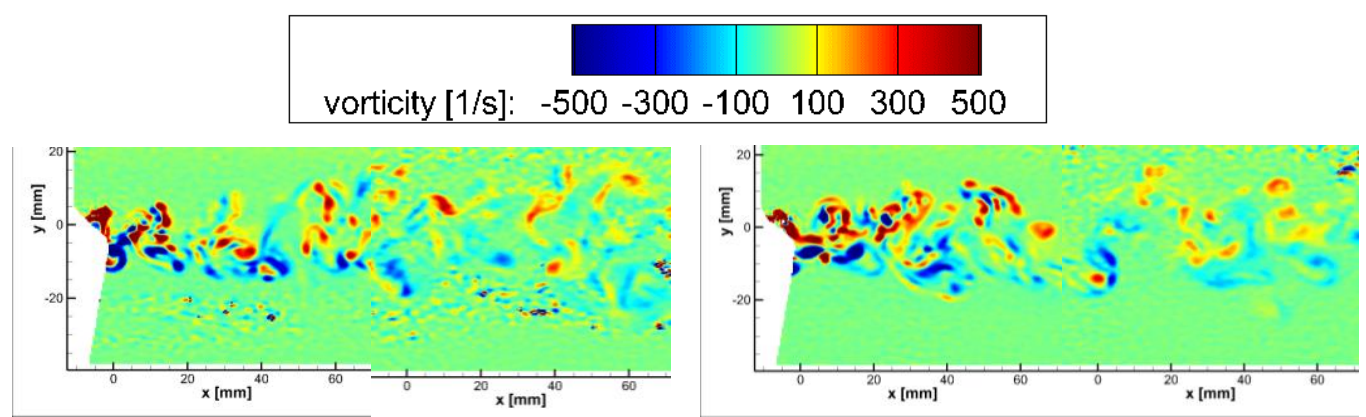

(a
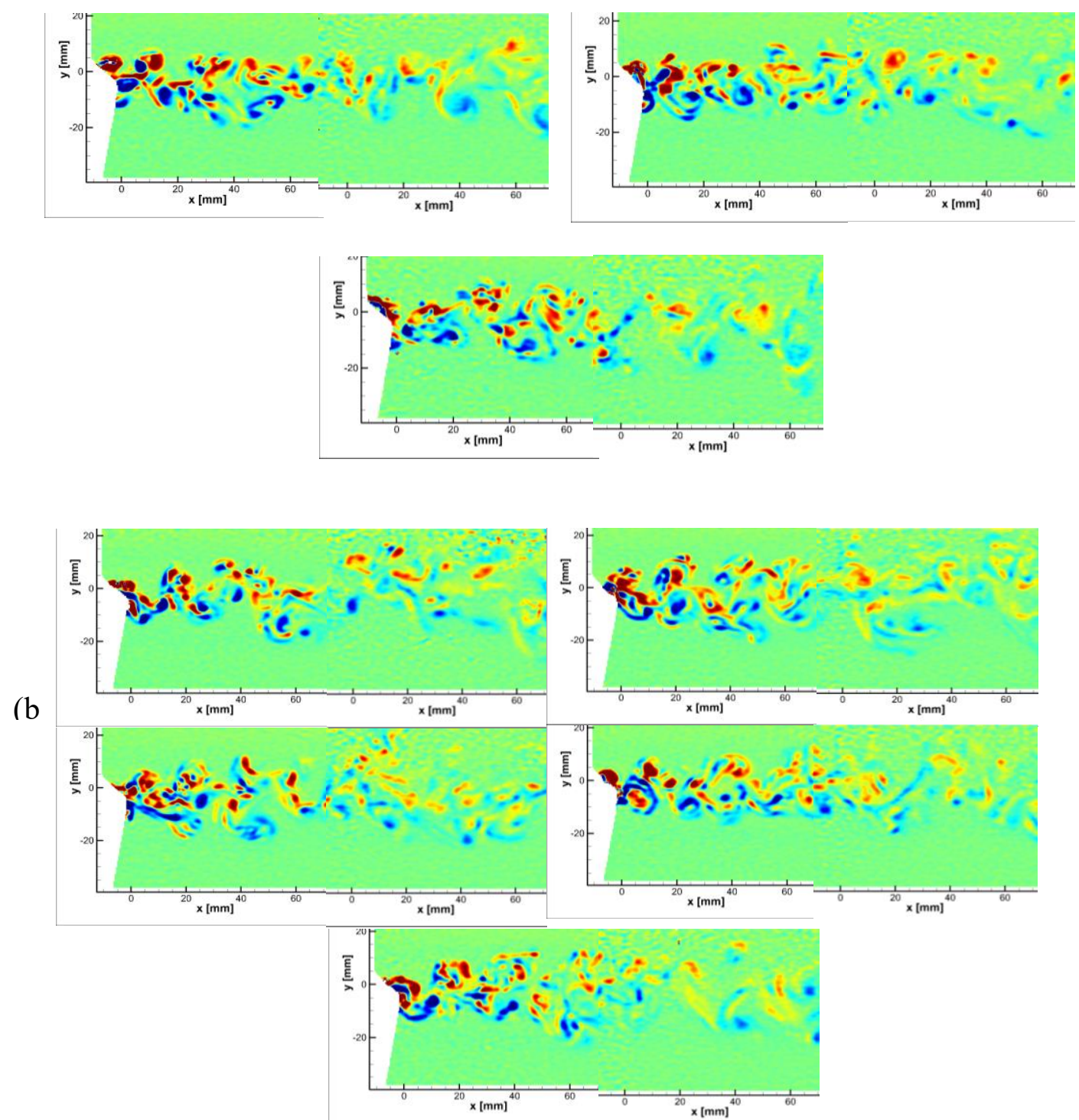

Figure G.1. Instantaneous vorticity plot when the cylinder orientation was (a) $0^{0}$ (b) $180^{\circ}$ at $\mathrm{Re}=57$ rotation $\mathrm{RPM}=960 \mathrm{~Hz}$ 

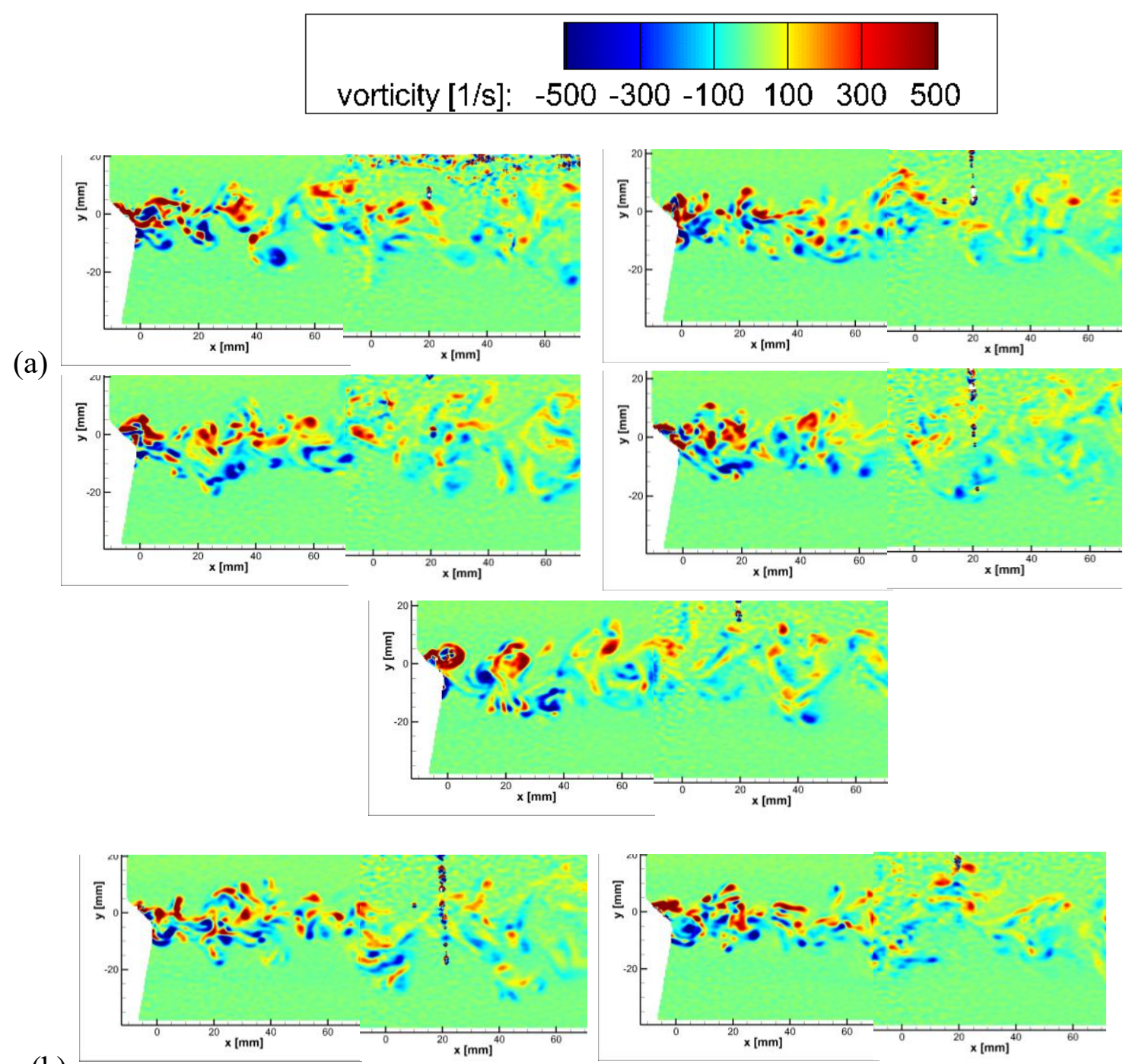

(b)
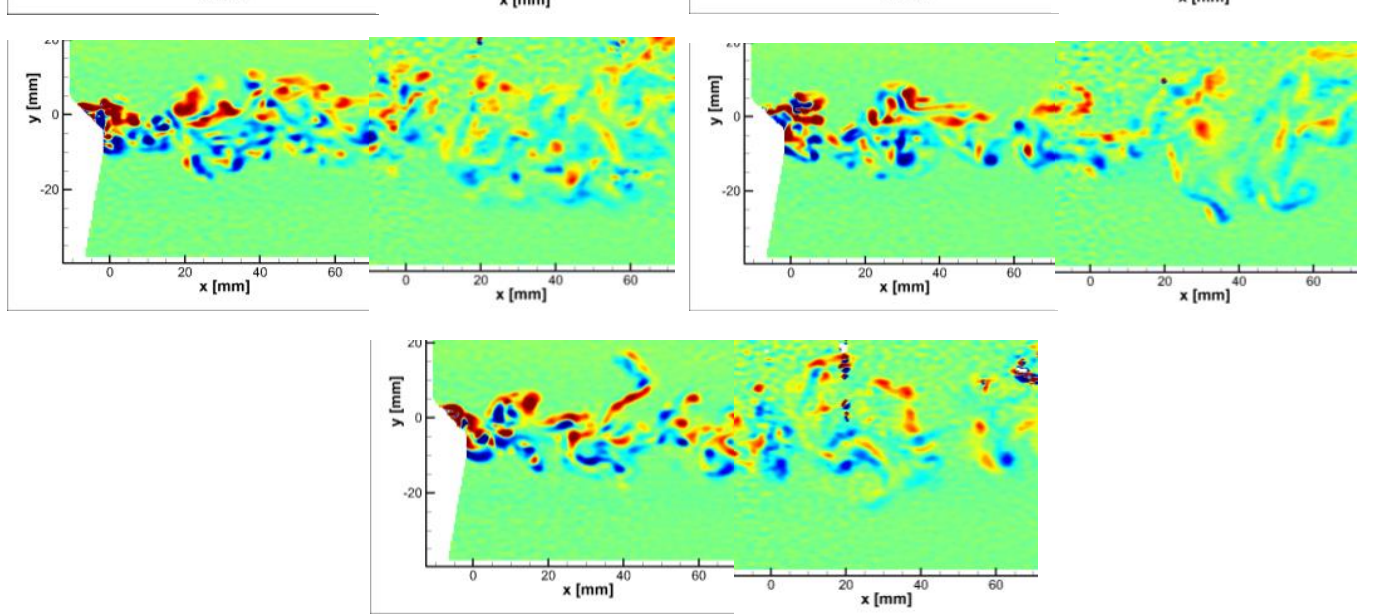

Figure G.2. Instantaneous vorticity plot when the cylinder orientation was (a) $0^{0}$ (b) $180^{\circ}$ at $\mathrm{Re}=57$ rotation $\mathrm{RPM}=1500 \mathrm{~Hz}$ 
(a)

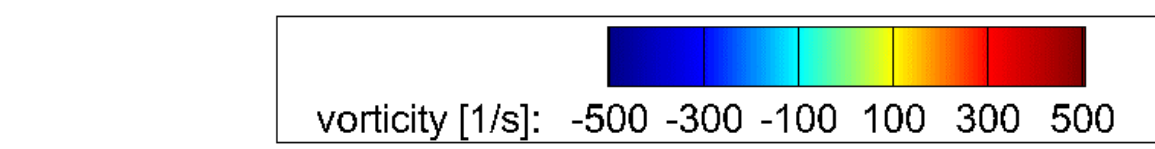

)
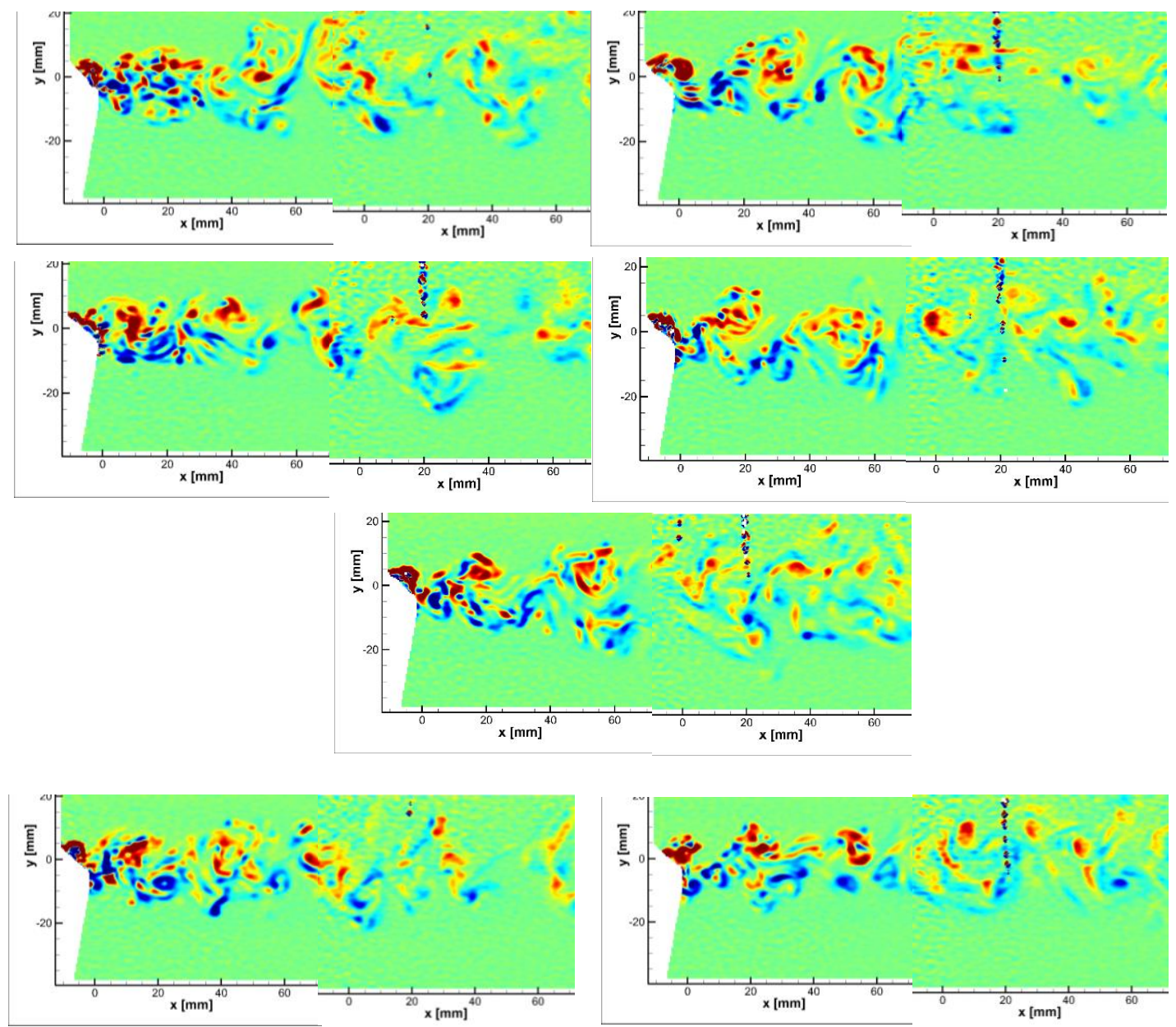

(b)
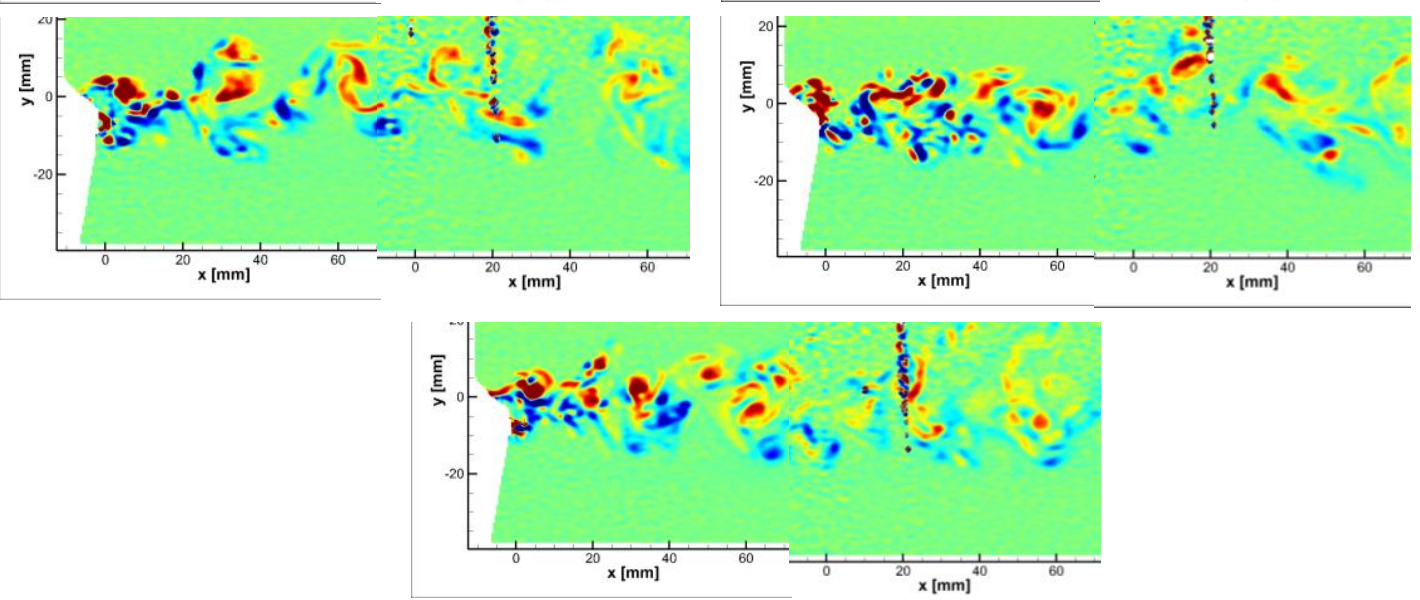

Figure G.3. Instantaneous vorticity plot when the cylinder orientation was (a) $0^{0}$ (b) $180^{\circ}$ at $\mathrm{Re}=57$ rotation $\mathrm{RPM}=1800 \mathrm{~Hz}$ 
(a)
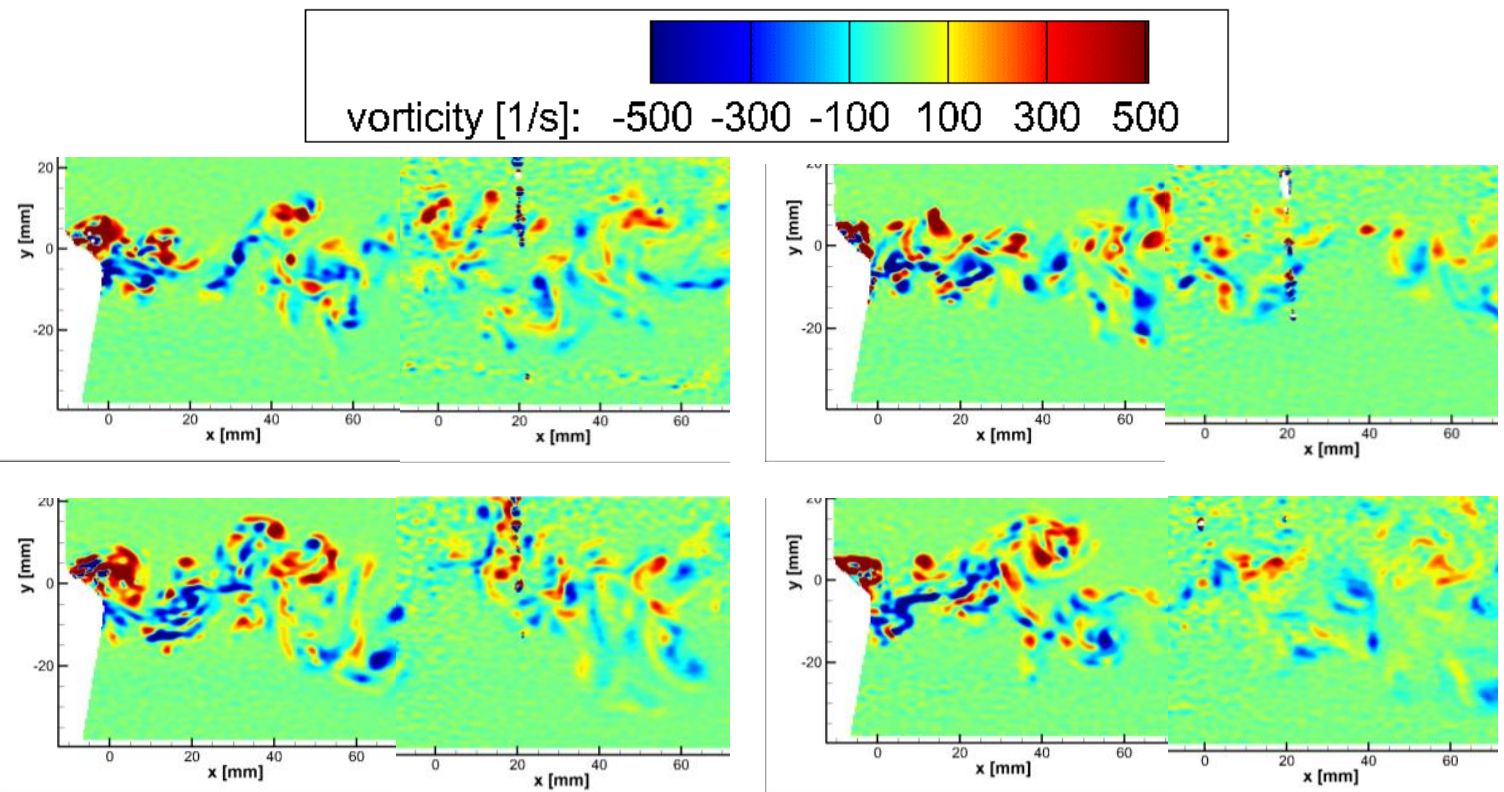

(b)
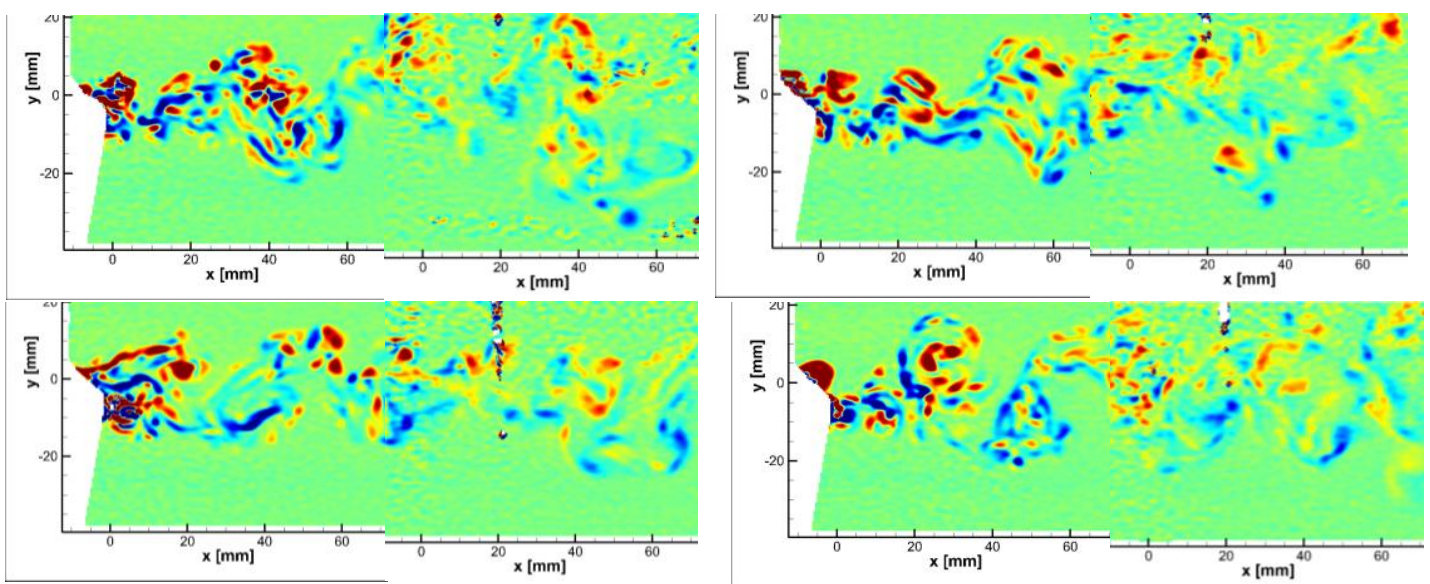

Figure G.4. Instantaneous vorticity plot when the cylinder orientation was (a) $0^{0}$ (b) $180^{\circ}$ at $\mathrm{Re}=57$ rotation $\mathrm{RPM}=2400 \mathrm{~Hz}$ 


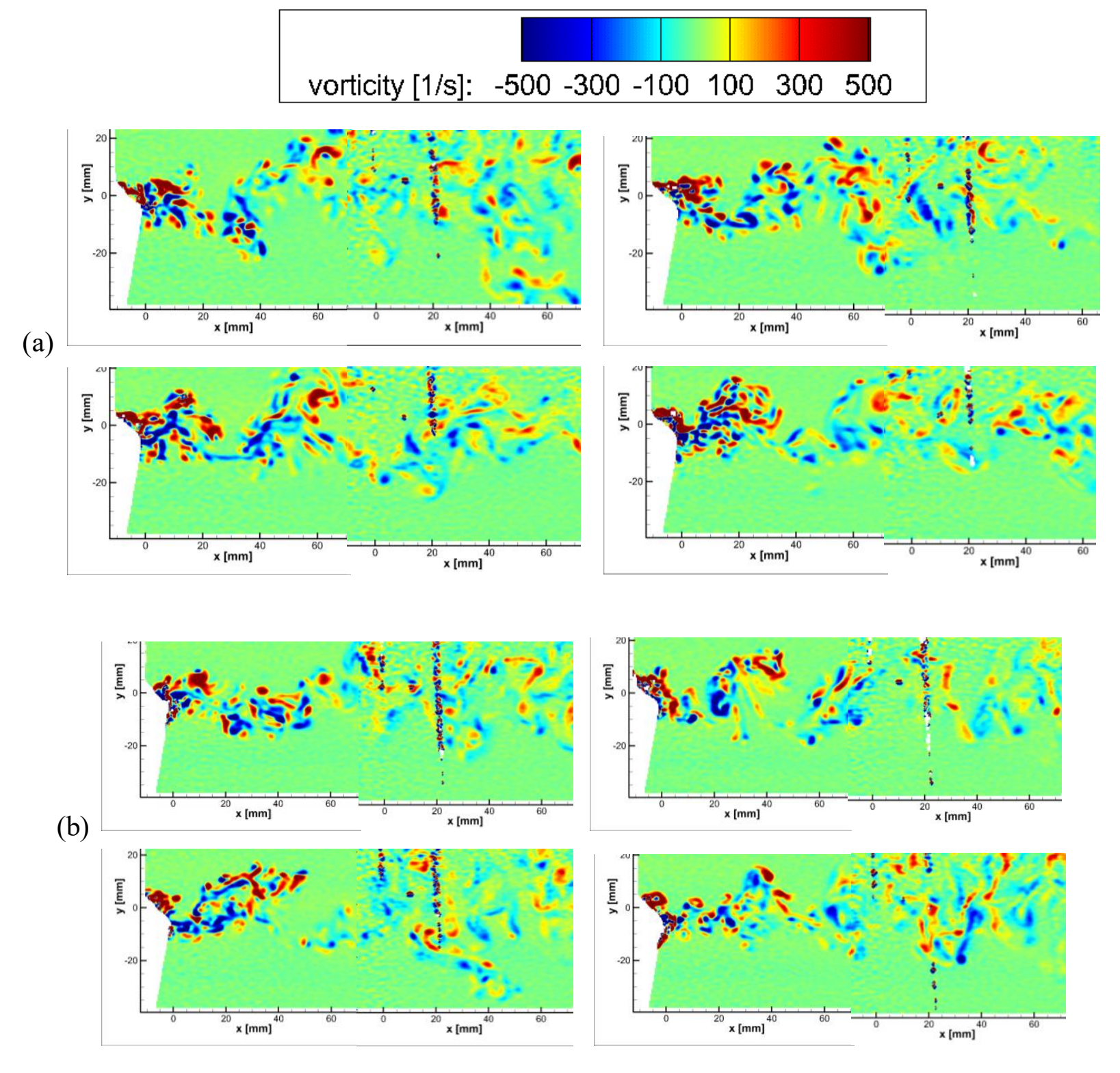

Figure G.5. Instantaneous vorticity plot when the cylinder orientation was (a) $0^{0}$ (b) $180^{\circ}$ at $\mathrm{Re}=57$ rotation $\mathrm{RPM}=3000 \mathrm{RPM}$ 


\section{APPENDIX H}

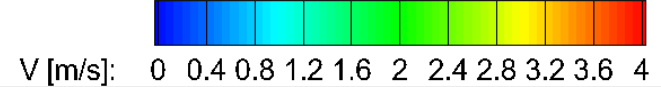

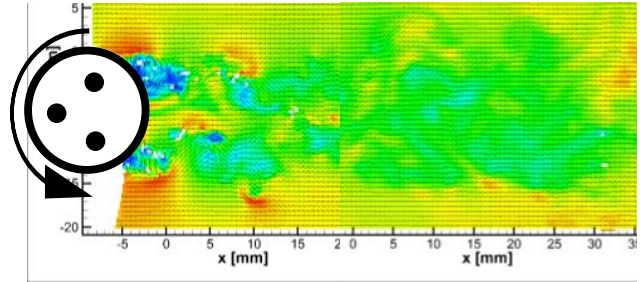

(a)

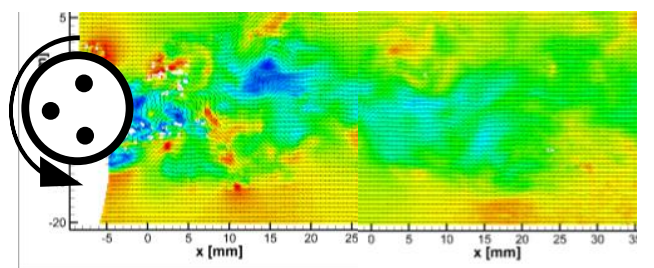

(c)

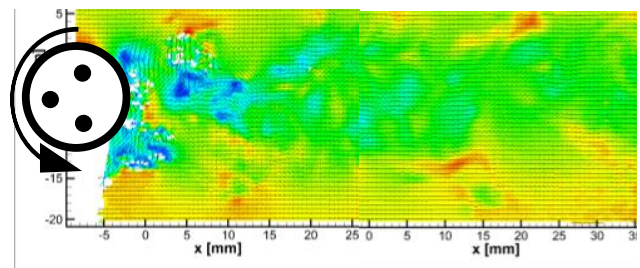

(e)

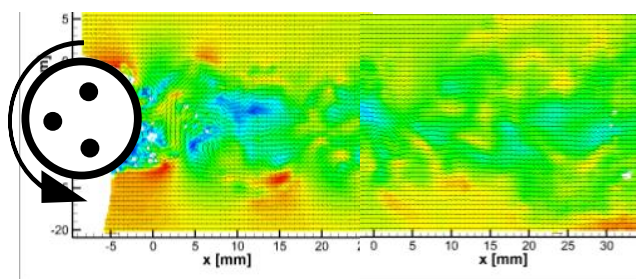

(b)

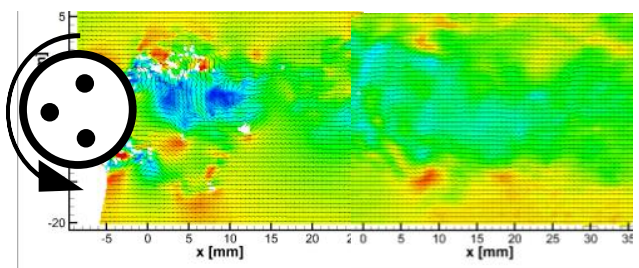

(d)

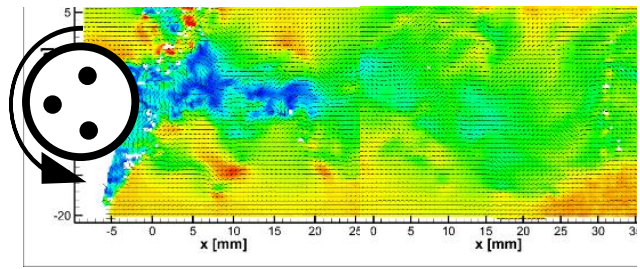

(f)

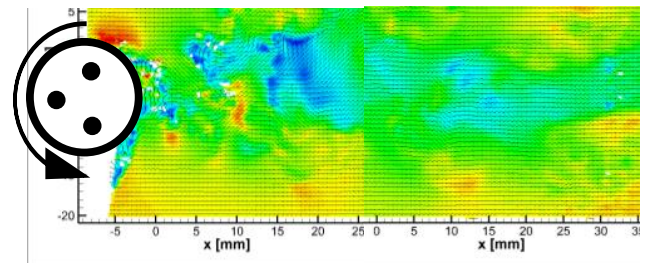

(g)

Figure H.1. Instantaneous Velocity Contour at $\mathrm{Re}=500$ and Rotation Speed is (a) 120 (b) 360 (c) 960 (d) 1500 (e) 1800 (f) 2400 (g) 3000 RPM 


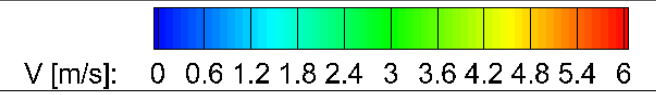

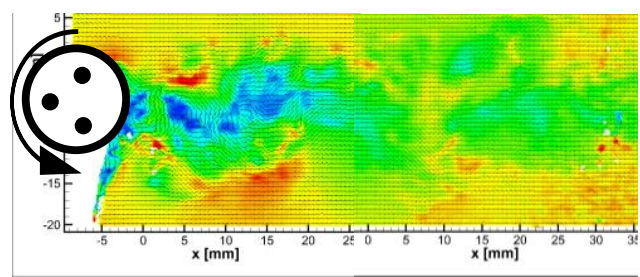

(a)

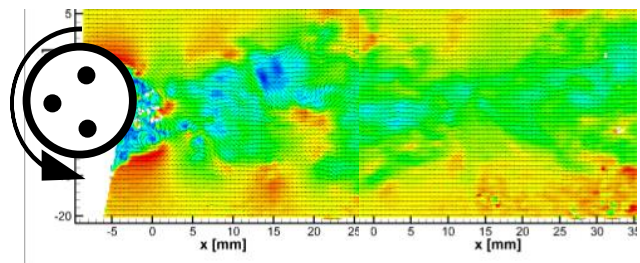

(c)

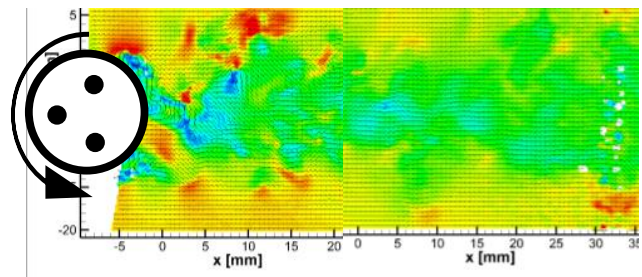

(e)

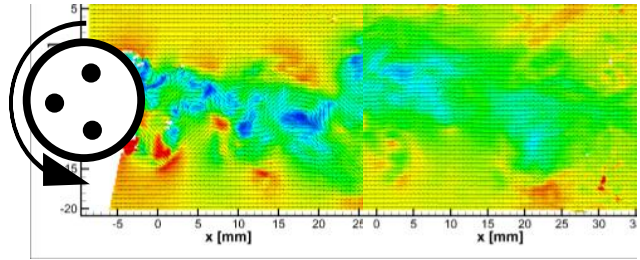

(b)

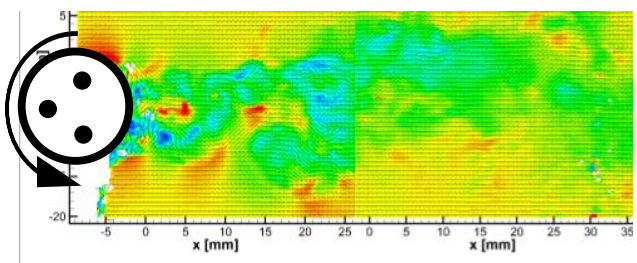

(d)

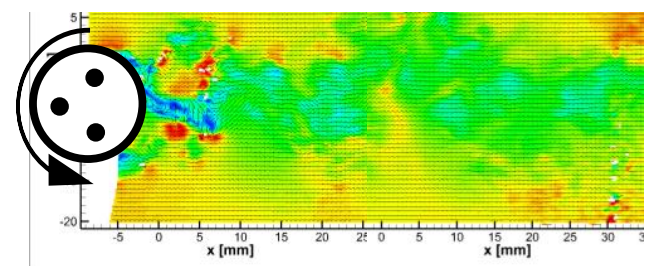

(f)

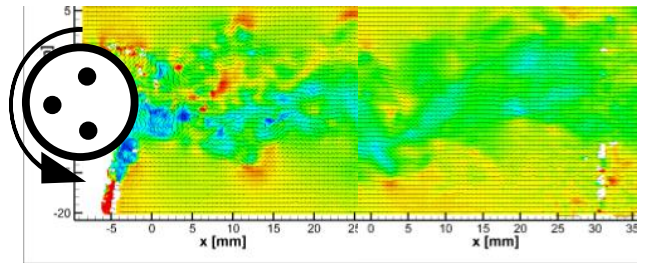

$(\mathrm{g})$

Figure H.2. Instantaneous Velocity Contour at $R e=858$ and Rotation Speed is (a) 120 (b) 360 (c) 960 (d) 1500 (e) 1800 (f) 2400 (g) 3000 RPM 


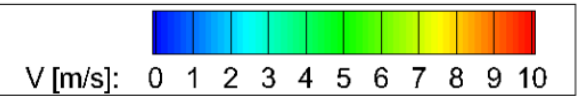

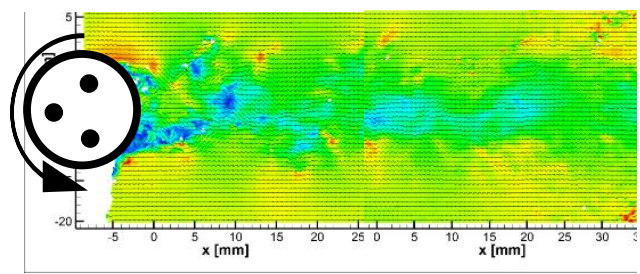

(a)

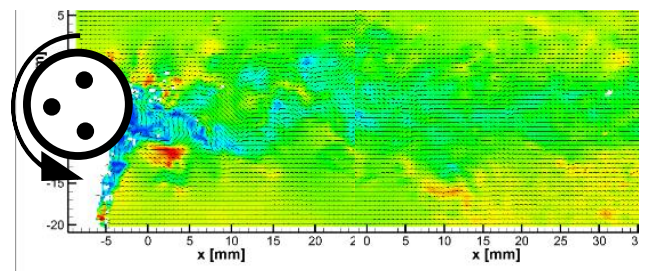

(c)

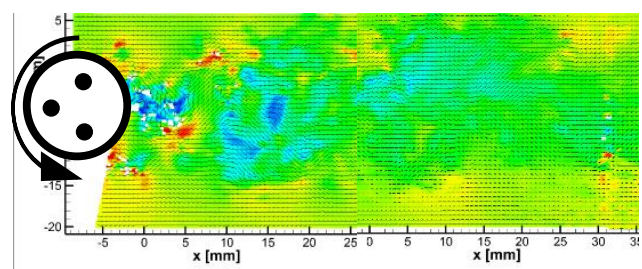

(e)

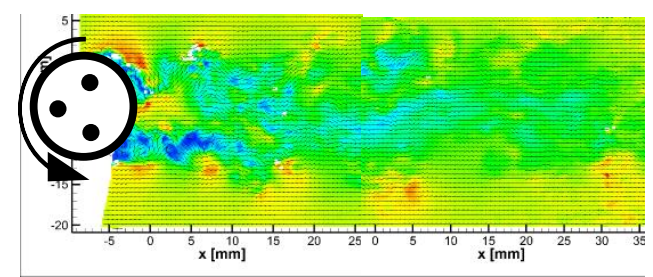

(b)

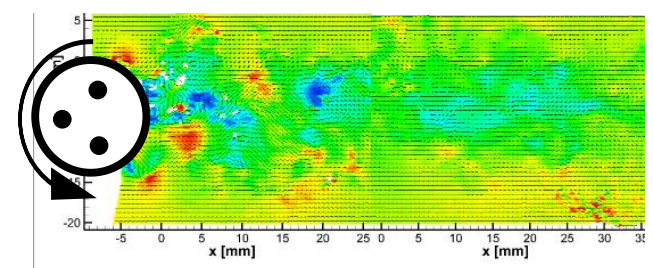

(d)

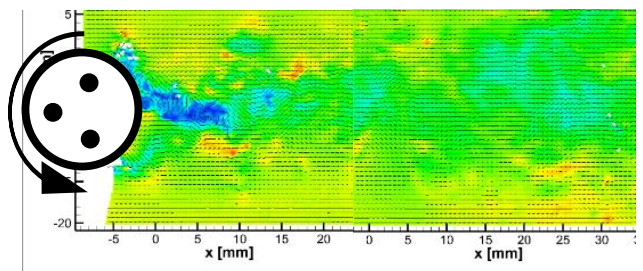

(f)

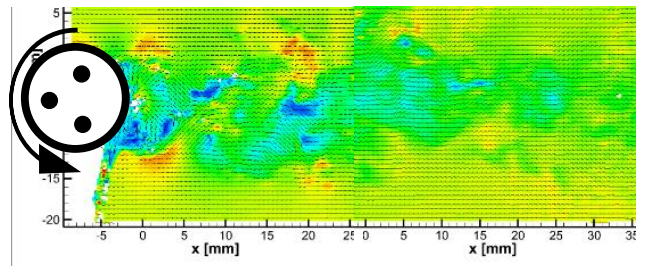

$(\mathrm{g})$

Figure H.3. Instantaneous Velocity Contour at $\mathrm{Re}=1250$ and Rotation Speed is (a) 120 (b) 360 (c) 960 (d) 1500 (e) 1800 (f) 2400 (g) 3000 RPM 